\title{
Mentale und physische Gesundheit im Alter
}

Der Einfluss von Armut,

Einkommensungleichheit und

Vermögensungleichheit 
Fabio Franzese

Mentale und physische Gesundheit im Alter 
„Poverty is a problem, or it is nothing. What it cannot be is not a problem." (Alcock 1993, 4; Hervorhebung im Original) 
Fabio Franzese

\section{Mentale und physische Gesundheit im Alter}

Der Einfluss von Armut,

Einkommensungleichheit und

Vermögensungleichheit

Budrich Academic Press GmbH

Opladen • Berlin • Toronto 2020 
Bibliografische Information der Deutschen Nationalbibliothek

Die Deutsche Nationalbibliothek verzeichnet diese Publikation in der Deutschen Nationalbibliografie; detaillierte bibliografische Daten sind im Internet über http://dnb.d-nb.de abrufbar.

Dissertation, Universität Heidelberg, 2019

(C) 2020 Dieses Werk ist bei der Verlag Budrich Academic Press GmbH erschienen und steht unter der Creative Commons Lizenz Attribution-ShareAlike 4.0 International (CC BY-SA 4.0): https:/creativecommons.org/licenses/by-sa/4.0/ Diese Lizenz erlaubt die Verbreitung, Speicherung, Vervielfältigung und Bearbeitung bei Verwendung der gleichen CC-BY-SA 4.0-Lizenz und unter Angabe der UrheberInnen, Rechte, Änderungen und verwendeten Lizenz.

\section{(c) (1) ()}

Dieses Buch steht im Open-Access-Bereich der Verlagsseite zum kostenlosen Download bereit (https://doi.org/10.3224/96665012).

Eine kostenpflichtige Druckversion kann über den Verlag bezogen werden. Die Seitenzahlen in der Druck- und Onlineversion sind identisch.

$\begin{array}{ll}\text { ISBN } & 978-3-96665-012-0 \\ \text { eISBN } & 978-3-96665-987-1 \\ \text { DOI } & 10.3224 / 96665012\end{array}$

Umschlaggestaltung: Bettina Lehfeldt, Kleinmachnow - www.lehfeldtgraphic.de Typografisches Lektorat: Anja Borkam, Jena - kontakt@lektorat-borkam.de Druckerei: Books on Demand $\mathrm{GmbH}$, Norderstedt Printed in Europe 


\section{Inhaltsverzeichnis}

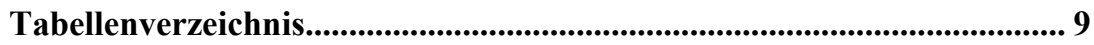
Abbildungsverzeichnis.

$1 \quad$ Einleitung................................................................................................... 13

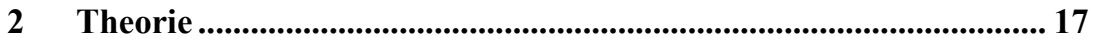

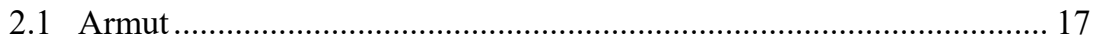

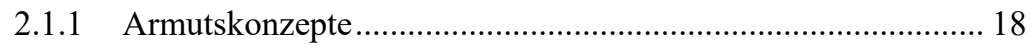

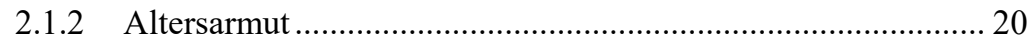

2.1.3 Mindestsicherung in Europa ................................................. 22

2.2 Gesundheitliche Ungleichheit ........................................................ 23

2.2.1 Verhalten als Erklärung für gesundheitliche Ungleichheit ....... 23

2.2.2 Psychosoziale Erklärung........................................................ 25

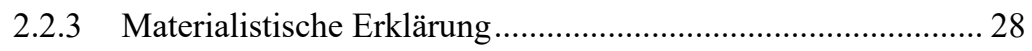

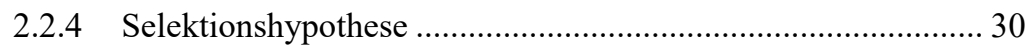

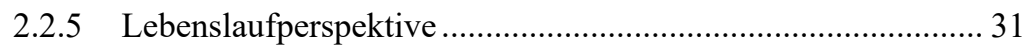

2.2.6 Der Einfluss von Armut auf Gesundheit................................. 33

2.2.7 Veränderungen in der finanziellen Situation ........................... 35

2.2.8 Einkommens- und Vermögensungleichheit............................ 37

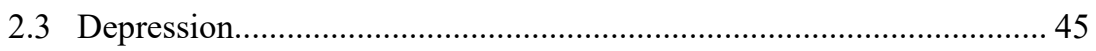

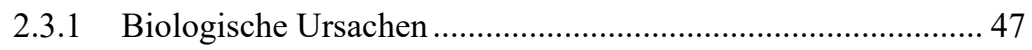

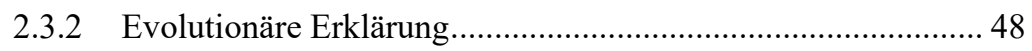

2.3.3 Genetische Ursachen ................................................................ 49

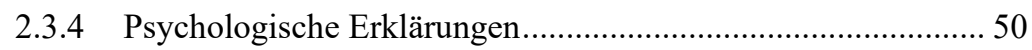

2.3.5 Soziale Erklärungen.................................................................. 51

2.3.6 Depression im Alter................................................................... 53

2.3.7 Einfluss der finanziellen Situation auf Depression.................... 55

2.4 Einschränkungen in den Aktivitäten des täglichen Lebens .................... 57

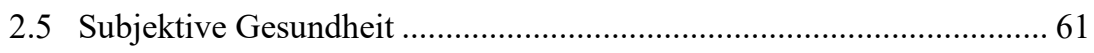




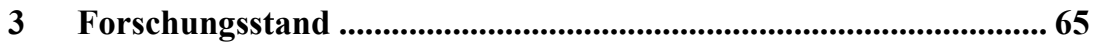

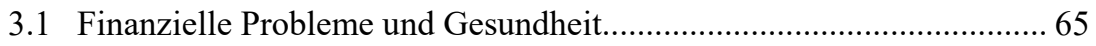

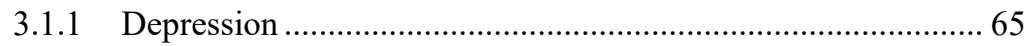

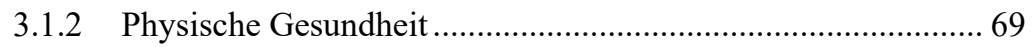

3.1.3 Einschränkungen in Aktivitäten des täglichen Lebens ............. 70

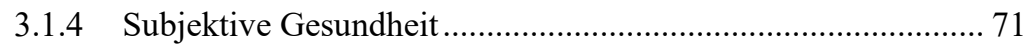

3.1.5 Vergleich von Quer- und Längsschnittanalysen ....................... 73

3.2 Verbesserung und Verschlechterung der finanziellen Situation............. 74

3.3 Einkommens- und Vermögensungleichheit und Gesundheit ................. 77

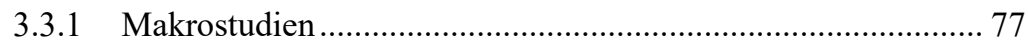

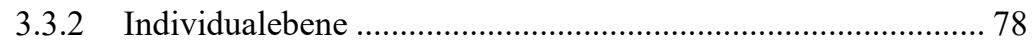

3.3.3 Zeitverzögerte Messung der Ungleichheit ................................ 81

3.3.4 Wer leidet unter der Ungleichheit? ............................................ 82

3.3.5 Kausale Effekte? .................................................................... 83

3.3.6 Tests der neomateriellen und psychosozialen Theorie ............. 86

3.3.7 Vermögensungleichheit ...................................................... 88

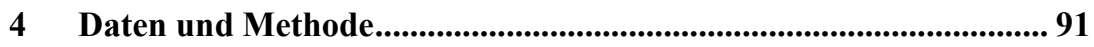

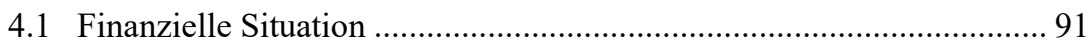

4.1.1 Finanzielle Schwierigkeiten................................................. 93

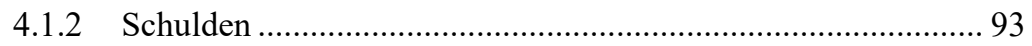

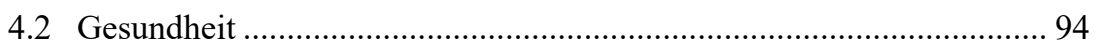

4.2.1 Depression ....................................................................... 94

4.2.2 Einschränkungen in den Aktivitäten des täglichen Lebens....... 95

4.2.3 Schlechte subjektive Gesundheit ........................................... 95

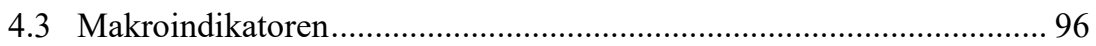

4.3.1 Einkommensungleichheit ..................................................... 96

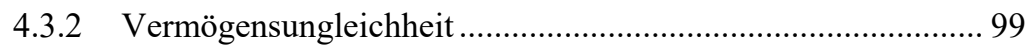

4.3.3 Soziales Vertrauen ................................................................. 99

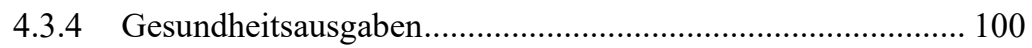

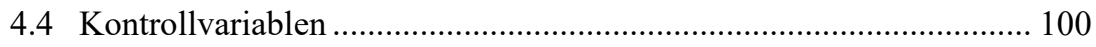

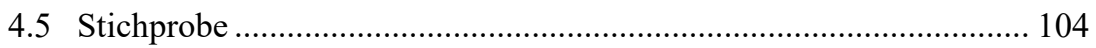

4.5.1 Veränderungen im Zeitverlauf.............................................. 108

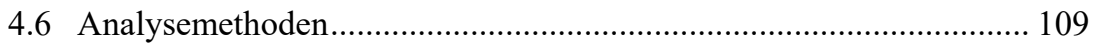


4.6.1 Pooled-OLS ........................................................................ 112

4.6.2 Random-Effects ................................................................ 113

4.6.3 Fixed-Effects ................................................................. 114

4.6.4 First-Difference ................................................................... 115

4.6.5 Hybrid-Modell ................................................................. 116

5 Analysen: Armut und Gesundheit ................................................. 119

5.1 Deskriptive Beschreibungen ........................................................... 119

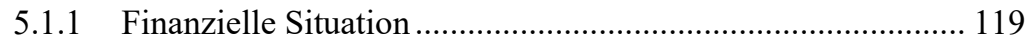

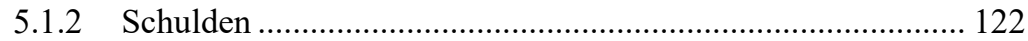

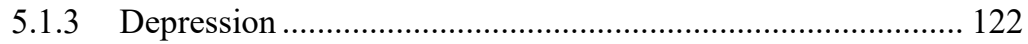

5.1.4 Einschränkungen in den Aktivitäten des täglichen Lebens..... 126

5.1.5 Schlechte subjektive Gesundheit ......................................... 127

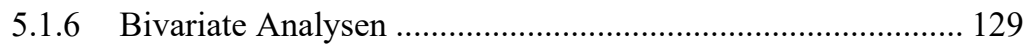

5.2 Vergleich von Querschnitt- und Längsschnittanalysen........................ 131

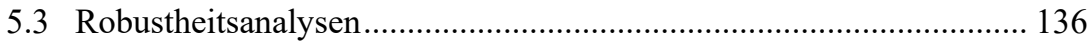

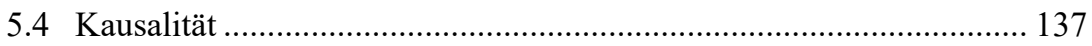

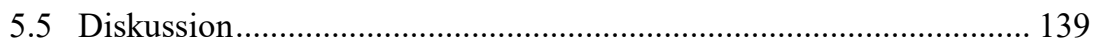

6 Analysen: Verbesserung und Verschlechterung der finanziellen Situation ................................................................................ 145

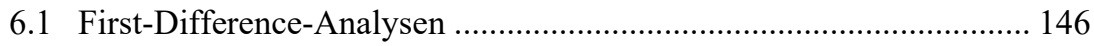

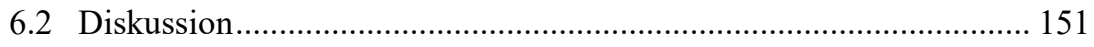

7 Analysen: Einkommens- und Vermögensungleichheit .................... 155

7.1 Deskriptive Beschreibungen .......................................................... 155

7.2 Multivariate Analysen................................................................. 162

7.2.1 Test der psychosozialen und neomateriellen Theorie............. 164

7.2.2 Interaktion: Wer leidet unter der Ungleichheit? .................... 165

7.3 Diskussion der Ergebnisse .............................................................. 170

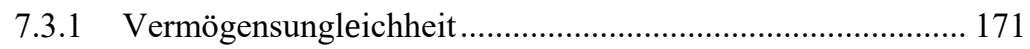

7.3.2 Einkommensungleichheit ................................................... 173

7.3.3 Theorievergleich............................................................... 175

7.3.4 Interaktion: Wer leidet unter der Ungleichheit? ..................... 176 


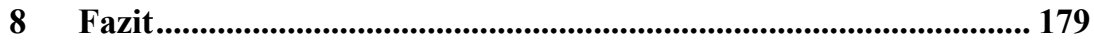

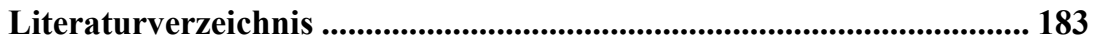

\section{Anhang}

Alle Anhänge stehen auf der Webseite des Verlags zum kostenlosen Download zur Verfügung: https://doi.org/10.3224/96665012A 


\section{Tabellenverzeichnis}

Tabelle 4.1: Übersicht aller SHARE Wellen und Länder............................ 92

Tabelle 4.2: Verteilung des Bruttoäquivalenzeinkommens in

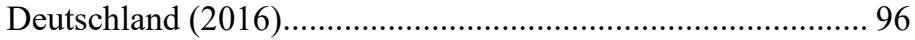

Tabelle 4.3: Zuordnung von ESS und SHARE Wellen ............................... 100

Tabelle 4.4: Beschreibung der Stichprobe ................................................ 106

Tabelle 4.5: Fallzahlen pro Land und Welle.............................................. 107

Tabelle 4.6: Veränderung in der finanziellen Situation der Haushalte ....... 109

Tabelle 4.7: Veränderung in der Gesundheit ............................................. 109

Tabelle 5.1: Anzahl depressiver Symptome in der Stichprobe (Welle 6)... 122

Tabelle 5.2: Fixed-Effects-Modelle mit und ohne Gesundheit als

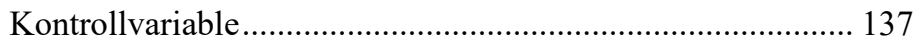

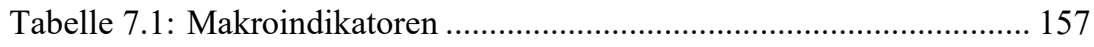

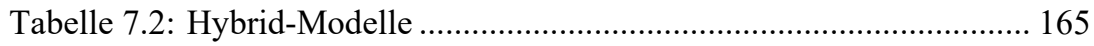




\section{Abbildungsverzeichnis}

Abbildung 2.1: Der Effekt von steigender Einkommensungleichheit auf die Mortalität

Abbildung 2.2: Mechanismen des Einflusses von Einkommensungleichheit auf Depression

Abbildung 4.1: Lorenzkurve des Bruttoäquivalenzeinkommens 2016

Abbildung 4.2: Schema für den direkten und indirekten Effekt von finanzieller Situation auf die mentale Gesundheit .......... 103

Abbildung 4.3: Generierungsprozess der Analysestichprobe 104

Abbildung 4.4: Fiktives Beispiel: Lineares Wahrscheinlichkeitsmodell und Logit-Modell

Abbildung 5.1: Finanzielle Situation der Haushalte in SHARE nach Land und Welle. 120

Abbildung 5.2: Schulden der Befragten in SHARE nach Land und Welle.

Abbildung 5.3: Prävalenz von Depression nach Land und Geschlecht (in Prozent, Welle 6)

Abbildung 5.4: Prävalenz von Depression nach Altersgruppe (in Prozent).

Abbildung 5.5: Prävalenz von Depression im Zeitverlauf nach Land (in Prozent)

Abbildung 5.6: Einschränkungen in Aktivitäten des täglichen Lebens nach Altersgruppe (in Prozent)

Abbildung 5.7: Einschränkungen in Aktivitäten des täglichen Lebens im Zeitverlauf nach Land (in Prozent)

Abbildung 5.8: Schlechte subjektive Gesundheit im Zeitverlauf nach Land (in Prozent) ........................................................ 128

Abbildung 5.9: Anteil der Personen mit Depression nach finanzieller Situation

Abbildung 5.10: Depression, Einschränkungen in ADL und schlechte subjektive Gesundheit nach Schulden und Welle ........... 130

Abbildung 5.11: Vergleich von OLS, POLS, RE und FE - Depression .... 132 Abbildung 5.12: Vergleich von OLS, POLS, RE und FE Einschränkungen in ADL und subjektive Gesundheit .... 133 
Abbildung 5.13: Vergleich von FE-Modellen mit unterschiedlicher Referenzkategorie der Variable ,finanzielle Probleme“. 135

Abbildung 5.14: Fixed-Effects Modelle. Vergleich der Gesamtstichprobe mit der Teilstichprobe „Rentner“...... 139

Abbildung 6.1: First-Difference-Modell - Effekte von Veränderung der finanziellen Schwierigkeiten auf Depression............ 146

Abbildung 6.2: $\quad$ First-Difference-Modell - Effekte von Veränderung der finanziellen Schwierigkeiten auf Einschränkungen in ADL

Abbildung 6.3: First-Difference-Modell - Effekte von Veränderung der finanziellen Schwierigkeiten auf schlechte subjektive Gesundheit.

Abbildung 6.4: First-Difference-Modell - Effekte von Veränderung der finanziellen Schwierigkeiten auf Schulden

Abbildung 7.1: Korrelation von Einkommensungleichheit (2015) und Vermögensungleichheit 158

Abbildung 7.2: Korrelation der Makroindikatoren ................................. 159

Abbildung 7.3: Korrelation von Gesundheits- und Makroindikatoren .... 160

Abbildung 7.4: Korrelation von Gesundheit und Einkommensungleichheit bei verzögerter Messung der Einkommensungleichheit.

Abbildung 7.5: Hybrid-Modell: Effekte von Veränderungen der finanziellen Situation nach Einkommensungleichheit: Depression 166

Abbildung 7.6: Hybrid-Modell: Effekte von Veränderungen der finanziellen Situation nach Einkommensungleichheit: Einschränkungen in ADL

Abbildung 7.7: Hybrid-Modell: Effekte von Veränderungen der finanziellen Situation nach Einkommensungleichheit:

Schlechte subjektive Gesundheit 168

Abbildung 7.8: Hybrid-Modell: Effekt von Verschuldung nach Einkommensungleichheit 169 


\section{Einleitung}

Obwohl Europa zu den wohlhabenden Regionen der Welt zählt, ist Armut ein Problem, das viele Menschen betrifft. Gemeint ist hierbei jedoch meist nicht die absolute Armut - also zu wenig Ressourcen um alle lebenswichtigen Grundbedürfnisse ausreichend zu befriedigen -, sondern die relative Armut im Vergleich zum Wohlstand der jeweiligen Gesellschaft. Die Quote der armutsgefährdeten Menschen, d.h. Personen in Haushalten mit einem Einkommen, das geringer ist als 60 Prozent des Medianeinkommens des entsprechenden Landes, lag im Jahr 2016 in der Europäischen Union bei 17,3 Prozent (Eurostat 2018a). Zusätzlich zu den Betroffenen, die unter den Problemen leiden, die Armut mit sich bringt, gibt es auch andere Teile der Bevölkerung, deren Leben durch die Angst vor Armut im Alter beeinträchtigt wird (vgl. Gasche/Lamla 2012). Die Auswirkungen der Armut für die Betroffenen sind vielfältig. Ein bedeutender Aspekt, in dem arme Menschen benachteiligt sind, ist die Gesundheit. Eine Vielzahl an Studien bestätigt, dass mit einem geringeren sozialen Status und geringerem Einkommen eine schlechtere Gesundheit einhergeht (Lampert 2016). Dies ist sowohl für physische, als auch für psychische Erkrankungen nachgewiesen worden. Mit geringem Einkommen sind z.B. erhöhte Risiken für Sterblichkeit (Martikainen/Valkonen/Moustgaard 2009) und Depressionen (Lorant et al. 2003) verbunden.

Das ökonomische Umfeld auf gesellschaftlicher Ebene stellt die Rahmenbedingungen dar, in denen die eigene finanzielle Lage erlebt und wahrgenommen wird. Neben dem allgemeinen Wohlstandsniveau einer Gesellschaft ist hierfür die Verteilung der ökonomischen Ressourcen von Bedeutung. Die Verteilung des Einkommens und Vermögens innerhalb einer Gesellschaft beschreiben die ökonomischen Ungleichheiten, denen die Mitglieder der Gesellschaft täglich ausgesetzt sind: die „Schere zwischen Arm und Reich“. Diese Ungleichheiten sind in Europa unterschiedlich stark ausgeprägt. In Spanien herrscht eine relativ große und in Tschechien eine relativ geringe Ungleichheit im Einkommen, Deutschland liegt im europäischen Vergleich im Mittelfeld (Eurostat 2016). Auch dieser gesellschaftliche Aspekt der finanziellen Lage kann sich auf die Gesundheit auswirken. Es existieren zahlreiche Studien, die zeigen, dass in Staaten, in denen das Einkommen ungleich verteilt ist, die Bevölkerung im Durchschnitt einen schlechteren Gesundheitszustand aufweist, als in Staaten mit geringerer Ungleichheit (Torre/Myrskylä 2014).

Die individuellen und gesellschaftlichen ökonomischen Bedingungen haben einen stärkeren Einfluss auf die Gesundheit, wenn der Körper über eine geringe Leistungsfähigkeit und ein schwaches Immunsystem verfügt (vgl. Kwetkat 2010). Da die Leistungsfähigkeit des Körpers mit zunehmendem Alter abnimmt und die Wahrscheinlichkeit zu erkranken ansteigt (vgl. Siegrist 2005: 215f; Andersen-Ranberg et al. 2009), ist die ältere Bevölkerung eine 
Gruppe mit erhöhtem Risiko für gesundheitliche Folgen, die aus Veränderungen in der finanziellen Situation resultieren. Neben der mit dem Alter abnehmenden Gesundheit (Leopold/Engelhardt/Engelhartdt 2013) unterscheidet sich das höhere Alter auch in anderen Aspekten von den Phasen des jungen und mittleren Erwachsenenalters. In diese Zeit fällt bspw. der Übergang vom Erwerbsleben in den Ruhestand, der mit vielen Änderungen verbunden ist, wie z.B. in den sozialen Kontakten, der Freizeit und im Einkommen.

Die Erforschung der Gesundheit im höheren Alter gewinnt zunehmend an Bedeutung, da in vielen Ländern Europas der Anteil der älteren Bevölkerung stetig gestiegen ist und auch weiterhin ansteigen wird. Schätzungen der Europäischen Union (EU) gehen von einem Anstieg der Bevölkerung ab 65 Jahren von 18,5 Prozent im Jahr 2014 auf 28,7 Prozent im Jahr 2080 aus (EU 2015). Ursachen der Alterung der Gesellschaft sind geringe Geburtenraten sowie die steigende Lebenserwartung, die u.a. aus der zunehmend besseren Gesundheitsversorgung resultiert (Klein 2016: 89; Hoffmann/Menning/Schelhase 2009). Die europäischen Gesellschaften müssen sich also darauf einstellen, dass es mehr alte Menschen gibt, die länger leben. Ein längeres Leben kann ein längeres gesundes Leben bedeuten (Kroll/Ziese 2009), dies hat jedoch nicht zwangsläufig zur Folge, dass es mit weniger oder kürzeren Krankheitsphasen einhergeht. Auch eine Verschiebung und Verlängerung der Phasen von Krankheit und Pflegebedürftigkeit kann die Folge sein (vgl. Murray et al. 2015; Westendorp/Kirkwood 2008). Mit dem Alter wandelt sich zudem die Art der Erkrankung, so treten z.B. Herz-Kreislauf-Erkrankungen sowie Einschränkungen des Bewegungsapparates mit zunehmendem Alter häufiger auf (Saß/Wurm/Ziese 2009).

Sowohl Armut, als auch Gesundheit im höheren Alter, sind - für die Betroffenen wie auch für die Gesellschaft - relevante und allgegenwärtige Themen. In der vorliegenden Arbeit werden diese Themen verknüpft, um die Einflüsse ökonomischer Faktoren auf die Gesundheit im Alter anhand von zwei Forschungsfragen empirisch zu analysieren. Erstens steht im Mittelpunkt der Untersuchung welche Auswirkungen eine Veränderung der finanziellen Situation im höheren Alter auf die Gesundheit hat. Im Gegensatz zu einem Großteil der bisherigen Forschung, werden hierzu Längsschnittmethoden angewendet. Eine Besonderheit ist hierbei die genaue Analyse der Effekte von Verbesserungen und Verschlechterungen in der finanziellen Lage. So können die negativen Auswirkungen einer Verschlechterung mit den positiven Effekten einer Verbesserung verglichen werden. Die zweite Forschungsfrage beschäftigt sich mit der ökonomischen Ungleichheit auf gesellschaftlicher Ebene: Wie hängt die Einkommens- und Vermögensungleichheit in einer Gesellschaft mit der Gesundheit zusammen? Es werden zwei Theorien getestet, die versuchen diesen Zusammenhang zu erklären. Zudem wird untersucht, ob die Veränderung der Ungleichheit einen Einfluss auf die Gesundheit hat und ob die gesundheitsschädlichen Effekte von Armut bei größerer Ungleichheit stärker sind als bei 
geringer Ungleichheit. Auch hier stellt v.a. die Längsschnittdimension einen innovativen Beitrag zur Forschungslandschaft dar. Außerdem wird der Zusammenhang von Vermögensungleichheit und Gesundheit analysiert, ein bislang kaum erforschter Bereich, der mit den hier verwendeten Gesundheitsindikatoren zum ersten Mal untersucht wird.

Die Weltgesundheitsorganisation definiert Gesundheit als einen „Zustand des vollkommenen physischen, geistigen und sozialen Wohlbefindens" (Kickbusch 1999: 275). Daher werden die Analysen der vorliegenden Untersuchung anhand der mentalen und der physischen Gesundheit durchgeführt. Neben der subjektiven Gesundheit werden mit Depression und körperlichen Einschränkungen bedeutende Dimensionen von Gesundheit betrachtet. Der Anteil der Menschen, die an Depression leiden wird weltweit auf ca. 4,4 Prozent geschätzt, mit steigender Tendenz (World Health Organization 2017c). In Europa machen psychische Störungen 20 Prozent der Krankheitslast aus, wobei dieser Anteil im Gebiet der Europäischen Union noch etwas höher liegt (World Health Organization 2012). Dennoch wird psychischen Problemen - v.a. im höheren Alter - von Ärzten relativ wenig Aufmerksamkeit geschenkt. Diagnosen und Behandlungen psychischer Krankheiten sind im Alter selten (Stoppe 2008), obwohl die Prävalenz von Depressionen und daraus folgenden Suiziden gegen Ende des Lebens ansteigt (Wolff/Tesch-Römer 2017; IHME 2017; Conwell/Duberstein/Caine 2002). Die physische Gesundheit ist für ein zufriedenes Leben von großer Bedeutung, wobei der Selbstständigkeit eine bedeutende Rolle zukommt. Körperliche Einschränkungen und das Angewiesensein auf Hilfe verringern die Lebensqualität beachtlich (vgl. Netuveli et al. 2006; Andersen et al. 2004). Die subjektive Gesundheit spiegelt alle Dimensionen der Gesundheit wider und eignet sich daher als allgemeines Gesundheitsmaß (vgl. Benyamini 2011).

In Kapitel 2 werden die theoretischen Grundlagen dargestellt, die für die Beantwortung der Forschungsfragen notwendig sind. Dazu werden verschiedene Armutskonzepte sowie das Problem der Altersarmut erläutert und mögliche Erklärungen für die Unterschiede in der Gesundheit zwischen Bevölkerungsgruppen mit unterschiedlichem sozioökonomischem Status dargestellt. Zudem werden die beiden gängigen Theorien zur Erklärung des Zusammenhangs von Gesundheit und der Ungleichverteilung der ökonomischen Ressourcen diskutiert sowie die theoretischen Modelle zur Entstehung von Depressionen und körperlichen Einschränkungen sowie der Beurteilung der subjektiven Gesundheit beschrieben. Kapitel 3 gibt einen Überblick über die Ergebnisse der bisherigen empirischen Studien, die im Zusammenhang mit den Forschungsfragen relevant sind. Anschließend werden in Kapitel 4 die methodischen Aspekte der vorliegenden Studie dargestellt: die Daten, die Indikatoren zur Messung der finanziellen Situation und Gesundheit, die Auswahl der Kontrollvariablen sowie die Beschreibung der Stichprobe und der angewandten Methoden. Die folgenden Kapitel behandeln jeweils eine der Forschungs- 
fragen, wobei diese anhand verschiedener Analysemethoden untersucht und anschließend diskutiert werden. In Kapitel 5 werden deskriptive Analysen der zentralen Variablen berichtet und der Zusammenhang von finanziellen Problemen und Gesundheit anhand von Quer- und Längsschnittanalysen erörtert. In Kapitel 6 werden Verbesserungen und Verschlechterungen in der finanziellen Lage genauer auf ihren Einfluss auf die Gesundheit hin untersucht, um zu testen, ob die negativen Effekte einer Verschlechterung stärker sind als die positiven Effekte einer Verbesserung. Kapitel 7 beschäftigt sich mit dem Zusammenhang von ökonomischer Ungleichheit in einer Gesellschaft und der Gesundheit der Bevölkerung. Hierzu kommen sowohl deskriptive, als auch multivariate Methoden zum Einsatz. Zum Schluss werden die wichtigsten Ergebnisse zusammengefasst und ein Fazit gezogen (Kapitel 8). 


\section{Theorie}

In diesem Kapitel werden die theoretischen Grundlagen dargestellt, die im $\mathrm{Zu}-$ sammenhang mit den aufgeworfenen Forschungsfragen von Bedeutung sind. Zunächst werden die wichtigsten Konzepte zur Beschreibung von Armut erläutert, wobei auch auf die Besonderheiten der Altersarmut eingegangen wird (Kapitel 2.1). Drauf folgend werden in Kapitel 2.2 die Erklärungsansätze für die Ungleichheit in der Gesundheit innerhalb von Gesellschaften thematisiert. Hierbei wird insbesondere der Zusammenhang von ökonomischen Ressourcen und Gesundheit dargelegt. Zudem werden Theorien erläutert, die gesundheitliche Unterschiede zwischen Gesellschaften anhand der Einkommens- und Vermögensungleichheit erklären. Die darauffolgenden Kapitel beschreiben die Grundlagen der Gesundheitsmaße, die in dieser Arbeit empirisch analysiert werden. Durch die ausführliche Darstellung aller möglichen Faktoren, die den Ausbruch einer Depression verursachen und beeinflussen können (Kapitel 2.3), wird deutlich, wie komplex diese Krankheit ist. Gleiches gilt auch für die körperlichen Einschränkungen in alltäglichen Aktivitäten (Kapitel 2.4), die, wie auch das Konstrukt der subjektiven Gesundheit (Kapitel 2.5), aus theoretischer Perspektive beleuchtet werden.

\subsection{Armut}

Armut ist ein Problem, an dem Menschen auch in reichen Ländern sterben (Galea et al. 2011; O'Sullivan/Howden-Chapman/Fougere 2012). Aber auch die weniger dramatischen Folgen von finanziellen Problemen sind durchweg negativ, dazu zählen z.B. verringertes Selbstbewusstsein (Mayhew/Lempers 1998; Lange/Byrd 1998; Whitbeck et al. 1991), mehr Streit und geringere Zufriedenheit in Beziehungen (Papp/Cummings/Goeke-Morey 2009; Hardie/Lucas 2010; Shim/Lee/Kim 2016), Stigmatisierung (Knabe/Fischer/ Klärner 2018) und Scham (Walker et al. 2013). Um der Frage dieser Arbeit nachzugehen, inwieweit finanzielle Schwierigkeiten die Gesundheit älterer Personen in Europa beeinflusst, soll zunächst geklärt werden was Armut ist und wie man sie messen kann. Dazu werden in diesem Kapitel verschiedene Ansätze der Armutsforschung knapp dargestellt. Anschließend wird diskutiert, wie sich die hier verwendete Messung der Armut in die vorgestellten Konzepte einordnen lässt und es wird auf die Besonderheiten bei der Betrachtung von Altersarmut eingegangen. Zum Schluss wird noch ein kurzer Überblick über die Systeme der sozialen Mindestsicherung in Europa gegeben und dargestellt welche Rolle zur Beseitigung von Armut sie spielen. 


\subsubsection{Armutskonzepte}

„Armut bezeichnet einen Zustand, in dem Menschen ihre Grundbedürfnisse Nahrungsaufnahme, Wohnen, eine den klimatischen Bedingungen angemessene Bekleidung, medizinische Basisversorgung - nicht befriedigen können (absolute, extreme bzw. existenzielle Armut) oder von der Beteiligung am sozialen, kulturellen bzw. politischen Leben ihres Landes weitgehend ausgeschlossen sind (relative Armut)." (Butterwegge 2018)

Es ist schwierig internationale Standards bei der Messung der Armut anhand absoluter Kriterien festzulegen. So sind bspw. warme Kleidung und beheizbarer Wohnraum je nach klimatischen Bedingungen mehr oder weniger von Bedeutung (vgl. Butterwegge 2018; Strengmann-Kuhn/Hauser 2012). Aber auch innerhalb einer Gesellschaft ist eine Definition von absoluter Armut kein leichtes Unterfangen (Brülle 2018: 14). Zum einen ist es aus praktischer Sicht schwierig den gesamten Besitz und Kapitalvermögen einheitlich zu messen und daher ist dies selten in den Definitionen zu finden. Zum anderen müsste die Armutsgrenze für jeden Haushalt nach individuellem Bedarf angepasst werden, was teilweise durch die Gewichtung nach Haushaltszusammensetzung erreicht werden kann (ebd.). Im internationalen Vergleich wird oft von absoluter Armut gesprochen, wenn eine Person weniger als 1,90 US-Dollar pro Tag zur Verfügung hat (World Bank 2017; United Nations 2017). Die ursprüngliche Armutsgrenze von 1 US-Dollar pro Tag wurde in den 1980er Jahren eingeführt und seitdem schrittweise an die aktuellen Gegebenheiten angepasst (Ferreira/Jolliffe/Prydz 2015). Diese Grenze ist willkürlich und soll lediglich das Ausmaß der katastrophalen Verhältnisse in den ärmsten Ländern der Welt darstellen (Strengmann-Kuhn/Hauser 2012).

Während bei der absoluten Armut das zum Überleben notwendige ,absolute Existenzminimum" betrachtet wird, geht es bei der relativen Armut um das ,soziokulturelle Existenzminimum“ (Hauser 2012: 124). Was genau hierunter zu verstehen ist hängt wiederum von der jeweiligen Gesellschaft ab. Da in entwickelten Ländern absolute Armut ,als nahezu überwunden betrachtet werden" (Hauser/Neumann 1992: 246) kann, wird Armut meist aufgrund relativer Indikatoren beschrieben (Strengmann-Kuhn/Hauser 2012). Bei relativen Armutskonzepten kann zwischen dem Ressourcenansatz und dem Lebenslagenansatz unterschieden werden (Hauser/Neumann 1992). Der Ressourcenansatz betrachtet nur ökonomische Ressourcen, die für das soziokulturelle Existenzminimum notwendig sind. Ein gängiges $\mathrm{Ma} ß$ ist hierbei die relative Einkommensarmut: Der Anteil der Bevölkerung, dessen Einkommen weniger als 50 oder 60 Prozent des Medianeinkommens eines Landes beträgt (Klein 2016: 263). Eine alternative Definition ist die Armut anhand staatlich festgelegter Grenzen zu bestimmen, bspw. durch den Bezug von Mindestsicherung (vgl. Brülle 2018: 16 f; Geyer 2014). 
Der von Neurath (1937) eingeführte Begriff der Lebenslage definiert die Armut umfassender, wobei auch immaterielle Aspekte der Lebenssituation von Bedeutung sind (Leßmann 2006: 33). Es gibt verschiedene Interpretationen und (Weiter-) Entwicklungen des Lebenslagenansatzes, die im Grundgedanken übereinstimmen, dass die Lebenslage durch die „Menge von Möglichkeiten, die ein Mensch bei der Gestaltung seines Lebens hat" (ebd.) gekennzeichnet ist. „Die Betonung liegt bei diesem Konzept stärker auf der tatsächlichen Versorgungssituation des Individuums und weniger auf den potentiellen Versorgungslagen“ (Hauser/Neumann 1992: 247). Nach Weisser beschreibt die Lebenslage den ,Spielraum, den die äußeren Umstände dem Menschen für die Erfüllung der Grundanliegen bieten, die er bei unbehinderter und gründlicher Selbstbesinnung als bestimmend für den Sinn seines Lebens ansieht" (Weisser 1957: 6 zitiert nach Leßmann 2006: 33). Ein ähnlicher (vgl. Leßmann 2006), ebenfalls weit gefasster, multidimensionaler Blick auf Armut beschreibt Sen mit dem Capability-Ansatz (Sen 1993; Sen 1992). Sen spricht von den Verwirklichungschancen (,capability set“), die jeder Mensch hat und meint damit alle potentiell erreichbaren Lebenssituationen (vgl. Leßmann 2006: 34). Um die Situation eines Menschen zu beschreiben genügt es daher nicht, nur seine Ressourcen zu betrachten, da es sich individuell unterscheidet, wie diese eingesetzt werden können. Es geht um die Verwirklichungschancen die einem Menschen zur Verfügung stehen, um das Leben erfolgreich zu gestalten. Die Konzepte der Verwirklichungschancen und der Lebenslage haben einige Gemeinsamkeiten, so ,stellen sie den Menschen als Handelnden in den Mittelpunkt und postulieren einen Einfluss seines Handlungsspielraums auf sein Wohlergehen“ (ebd.: 31). Zudem ist die empirische Erfassung beider Ansätzen ein schwieriges Unterfangen, da die individuellen multidimensionalen Handlungsspielräume nicht ohne weiteres messbar sind (ebd.).

Ein Ansatz, der ebenfalls den Lebensstandard betrachtet und somit die Armut auch multidimensional beschreibt, jedoch empirisch besser greifbar ist als Verwirklichungschancen und Lebenslage, ist das Konzept der Deprivation (siehe Groenemeyer/Ratzka 2012; Brülle 2018: 14). ${ }^{1}$ Hierbei werden Kriterien bestimmt, die für ein Leben ohne Armut notwendig sind, bspw. warme Mahlzeiten, eine beheizbare Wohnung, die Möglichkeit Freunde zum Essen einzuladen und neue Kleidung zu kaufen (Townsend 1979; Nolan/Whelan 2010; Adena/Myck/Oczkowska 2015). Auch Bedingungen in der Wohngegend können relevant sein, z.B. die Verfügbarkeit von Schulen, Ärzten und Einkaufsmöglichkeiten (Mabughi/Selim 2006). Aus den abgefragten Lebensumständen kann ein absolutes (z.B. Armut entspricht mindestens zwei nicht zutreffende Kriterien) sowie ein relatives (Anteil der nicht zutreffenden Kriterien) Maß der Deprivation gebildet werden (Brülle 2018: 14).

1 Zur Beschreibung von Deprivation gibt es unterschiedliche Begriffe (relative Deprivation, soziale Deprivation, soziale Exklusion), die ähnliche Konzepte beschreiben, siehe dazu (Mabughi/Selim 2006). 
Zur Bestimmung von Armut bzw. Armutsgrenzen kann auch auf subjektive Kriterien zurückgegriffen werden (vgl. Piachaud 1992). Wird eine Person danach befragt, ob sie sich arm fühlt, dann werden unterschiedliche Bedürfnisse, Lebensweisen und Lebensumstände berücksichtigt. Dieselbe objektive ökonomische Lage kann von manchen Menschen als Armut erlebt werden, während andere sich selbst nicht als arm bezeichnen würden. Dieser Vorteil ist jedoch auch gleichzeitig ein Nachteil, denn mit der Subjektivität sind auch unterschiedliche Maßstäbe der Befragten verbunden und eine Vergleichbarkeit kann zumindest angezweifelt werden. Der Indikator, der in dieser Studie verwendet wird bezieht sich auf eine subjektive Einordnung der finanziellen Situation des Haushaltes. Es wird hierbei aber nicht nach Armut gefragt, sondern nach dem Ausmaß der Probleme, mit dem verfügbaren Einkommen über die Runden zu kommen (siehe Kapitel 4.1.1).Wird von solchen Problemen berichtet, so wird dies in manchen Studien als „subjektive Armut" bezeichnet (Adena/Myck 2014; Fonseca et al. 2013). Dieser Begriff ist durchaus gerechtfertigt, da in diesem Fall ein Haushalt nach eigener Angabe finanzielle Schwierigkeiten hat das Leben zu bestreiten. Es wird zwar nur nach Einkommen gefragt, aber für was das Geld benötigt wird, um ,über die Runden zu kommen“ ist nicht festgelegt. Auch Kosten für den Sportverein des Kindes oder andere Aktivitäten können in den Ausgaben enthalten sein. Somit enthält der Indikator implizit die Multidimensionalität vieler Armutskonzepte (welche Ausgaben zu ,über die Runden kommen" dazugezählt werden hängt von den Befragten ab). Zudem enthält die Frage auch indirekt eine Auskunft über das Vermögen. Denn ob ein bestimmtes Einkommen für den Lebensunterhalt ausreicht, hängt auch davon ab, ob davon Miete gezahlt werden muss oder man in der eigenen Wohnung lebt und ob anderes Vermögen vorhanden ist, das aufgebraucht werden kann. Auch nach dem Verständnis der Ansätze, die nach Lebenslagen und Verwirklichungschancen beurteilen, können große und kleine finanzielle Schwierigkeiten als Armut aufgefasst werden, da dadurch die Handlungsfähigkeit eingeschränkt wird. Dies geschieht nicht nur durch die Einschränkung im Konsum, sondern auch durch die mit finanziellen Problemen auftretenden Sorgen, welche „mentale Ressourcen“ beanspruchen und somit Konzentration und Leistungsfähigkeit negativ beeinflussen können (Mani et al. 2013).

\subsubsection{Altersarmut}

Obwohl die Armutsgefährdungsquote in Deutschland bei den jungen Erwachsenen am höchsten und im Rentenalter relativ gering ist (Statistisches Bundesamt 2018), steigt seit einiger Zeit die Angst vor Altersarmut immer weiter an (Gasche/Lamla 2012). In einer Studie zeigt sich, dass viele der Befragten zu pessimistisch sind und ihre zukünftige Rente zu gering einschätzen (Lamla/Gasche 2013). Dennoch sind diese Sorgen nicht ganz unbegründet, da 
die zukünftigen Rentner durch die Zunahme prekärer Beschäftigung und unterbrochenen Erwerbsbiographien geringere Rentenansprüche vorweisen können (Börsch-Supan/Gasche 2010; Haan et al. 2017; Kistler/Trischler 2012). In der öffentlichen Debatte wird daher oftmals ein Bild gezeichnet, das Alter mit Armut verknüpft. Auf die gleiche Weise wird Armut zum Teil auch als ein Problem in andern Gruppen angesehen, z.B. werden damit Arbeitslose, Obdachlose und Alleinerziehende in Verbindung gebracht (Leisering/Buhr 2012: 147). In dieser Betrachtung gilt Armut als eine soziale Lage, in der sich die Betroffenen dauerhaft befinden (Buhr 1995; Leisering/Buhr 2012). Seit den 1990er Jahren wird jedoch verstärkt ,dynamische“ Armutsforschung betrieben. Hierbei werden individuelle Lebensläufe analysiert und Armut als eine Episode im Leben betrachtet, die auch nur kurze Zeit andauern kann (GrohSamberg 2010; Buhr 1995; Leisering/Buhr 2012). Was die Dauer der Armutsphasen angeht, so unterscheidet sich das höhere Alter von früheren Lebensphasen. Im höheren Alter ist das Risiko höher, dass Episoden von Armut länger anhalten oder gar nicht überwunden werden können (Bourcarde 2012; Kümpers/Alisch 2018: 598). Sobald man nicht mehr am Erwerbsleben teilnimmt wird das Einkommen durch Rentenansprüche und frühere Ersparnisse bestimmt. Im Gegensatz zu früheren Lebensabschnitten, in denen auch bei Armut die Perspektive auf Verbesserung besteht, ist die Zukunftsaussicht im Alter weniger optimistisch (Bourcarde 2012). Weiterhin Geld anzusparen ist für die meisten kaum möglich und Erwerbstätigkeit im Ruhestand ist zwar möglich, jedoch sinkt die Erwerbsfähigkeit mit zunehmendem Alter (ebd.).

Im Erwerbsleben gibt es eine ganze Reihe von Gründen für eine Veränderung im Einkommen - z.B. Arbeitslosigkeit, Beförderung, Wechsel des Arbeitgebers und schließlich der Eintritt in die Rente. Wie bereits erwähnt bleibt das individuelle Einkommen ab dem Renteneintritt relativ konstant, es sei denn man geht zusätzlich einer Arbeit nach. Betrachtet man jedoch das Einkommen auf Haushaltsebene, so kommen verschiedene Lebensereignisse in Frage, die die finanzielle Situation des Haushaltes beeinflussen können. Ein- und Auszug sowie Veränderungen in der Erwerbssituation des Partners spielen eine wichtige Rolle. Vor allem Personen, die selbst ein geringes Einkommen haben und durch den Partner mitversorgt werden, sind stark gefährdet für Altersarmut. Sie spüren die negativen Auswirkungen von Trennung oder Tod des Partners besonders stark und können dadurch in die Armut abrutschen. Frauen sind diesem Risiko in besonderem Maße ausgesetzt, da sie oftmals aufgrund von Erwerbsunterbrechungen und Teilzeitarbeit relativ geringe Renten beziehen und zudem eine höherer Lebenserwartung haben als Männer (vgl. Butterwegge/Hansen 2012). Andere Szenarien für eine Veränderung der finanziellen Lage im Ruhestand sind Erbschaften und finanzielle Unterstützung durch die Familie. Betrachtet man nicht nur das Einkommen, sondern auch die Ausgaben, so sind mit zunehmendem Alter vor allem Kosten für die Gesundheit und Pflege ein bedeutendes Thema. 


\subsubsection{Mindestsicherung in Europa}

In vielen Staaten sind die Bürger für verschiedene Risiken über staatliche Systeme abgesichert. Dazu zählt Krankheit und Arbeitslosigkeit. Ein letztes Sicherheitsnetz, das vor dem Fall in die absolute Armut schützen soll, ist die Mindestsicherung. In allen Ländern der EU existiert ein solches allgemeines System zur Mindestsicherung, wobei diese in Ungarn und Griechenland erst vor kurzem eingeführt wurden (MISSOC 2018). Neumann (2015) beschriebt und vergleicht die Systeme der sozialen Mindestsicherung in allen EU-Staaten zum Stand des Jahres 2011. Aus der Studie geht hervor, dass die Systeme in den EU-Ländern in vielerlei Hinsicht sehr unterschiedlich sind. Ein Aspekt davon ist auch, dass Griechenland zu diesem Zeitpunkt kein Mindestsicherungssystem hatte. In den einzelnen Ländern gibt es verschiedene bedarfsbezogene Leistungen, zum Teil ist die Höhe der Bezüge aber auch vom Alter abhängig. „Mehr als ein Drittel der EU Staaten gewährt jungen Menschen geringere Leistungen als älteren. Luxemburg verwehrt ihnen den Zugang sogar komplett“ (ebd.: 434f.). Was die Generosität der Leistungen betrifft, so stehen Großbritannien und Irland an erster Stelle, dahinter folgen Dänemark und Luxemburg. Die geringsten Leistungen werden in Rumänien und Bulgarien gewährt. „Insgesamt betrachtet zeichnet sich die soziale Mindestsicherung durch große Unterschiede bei der durchschnittlichen Leistungshöhe in den europäischen Wohlfahrtsstaaten aus, die sich nicht in das bekannte Muster der klassischen Sozialstaatstypen einfügen“ (ebd.: 429f.). Dasselbe gilt auch für die Effektivität in Bezug auf Armutsbekämpfung, die Zugangs- und Bezugsbedingungen sowie die soziale Gerechtigkeit. Die Effektivität der sozialen Mindestsicherung bei der Armutsbekämpfung ist im Durchschnitt über alle EU-Länder nicht ausreichend. In Europa besteht trotz Bezug von Mindestsicherung das Risiko arm zu sein. Im europaweiten Durchschnitt liegt die Mindestsicherung 11 Prozent unter der Armutsrisikogrenze (60\% des Medianeinkommen) (ebd.: 434). In nur 6 der 27 Länder sind die Mindestsicherungsleistungen in der Lage das Armutsrisiko zu beseitigen: in Polen, Irland, Großbritannien, Tschechien, Dänemark und Estland. Deutschland liegt auf einem Niveau mit Litauen und den Niederlanden knapp unter dem EU-Durchschnitt. Mit Abstand am schlechtesten vor Armut schützt die Mindestsicherung in Bulgarien und Rumänien. Hier beträgt die Mindestsicherung nur ein Drittel bzw. die Hälfte des 60\% Medianeinkommens (ebd.: 436). 


\subsection{Gesundheitliche Ungleichheit}

Die gesundheitlichen Unterschiede zwischen Bevölkerungsschichten sind vielfach belegt worden (z.B. Jürges 2009; Huijts/Eikemo/Skalická 2010; Semyonov/Lewin-Epstein/Maskileyson 2013). Es gibt viele mögliche Mechanismen, die den sozioökonomischen Status und die Gesundheit verknüpfen. In der Diskussion stehen unter anderem „die Arbeitsbedingungen, die Wohnsituation, die soziale Sicherung, die soziale Integration und Teilhabe, das Gesundheitsverhalten, das Stressgeschehen, die Verfügbarkeit von sozialen und personalen Ressourcen sowie die Inanspruchnahme von Angeboten und Leistungen der Gesundheitsversorgung“ (Lampert 2016: 127). In diesem Kapitel werden allgemeine Theorien für die Entstehung von gesundheitlicher Ungleichheit vorgestellt. Orientiert an Bartley (2017) werden zuerst drei Erklärungsansätze dargestellt, mit denen die Entstehung von gesundheitlicher Ungleichheit in verschiedenen Statusgruppen beschrieben werden kann: Gesundheitsverhalten, die materialistische Theorie und die psychosoziale Theorie. Anschließend wird diskutiert, wie sich Gesundheit und sozialer Status gegenseitig beeinflussen und dadurch kausale Wirkmechanismen in beide Richtungen entstehen. Daran anknüpfend erläutert der folgende Abschnitt die Lebenslaufperspektive, denn besonders für die Gesundheit im Alter ist die Betrachtung des gesamten Lebenslaufes von Bedeutung. Im letzten Abschnitt wird beschrieben, wie die Einkommens- und Vermögensungleichheit einer Gesellschaft die Gesundheit von Individuen beeinflussen kann.

\subsubsection{Verhalten als Erklärung für gesundheitliche Ungleichheit}

Es ist empirisch umfangreich belegt, dass ein niedriger sozialer Status mit ungesünderem Verhalten einhergeht. Empirische Evidenz belegt diesen Zusammenhang für das Rauchverhalten (Lampert 2011; Pulkki et al. 2003; Aro/Avendano/Mackenbach 2005; Lampert/Kroll 2006), sportliche Aktivität (Klein 2009; Becker/Schneider 2005; Becker et al. 2007; Pulkki et al. 2003; Aro/Avendano/Mackenbach 2005), Gemüse- und Obstkonsum (Irz et al. 2014; Pérez 2002; Nicklett/Kadell 2013) und gesunde Ernährung (Schneider/ Schneider 2009b; Irz et al. 2014; Venn/Strazdins 2017; Lallukka et al. 2007). Zudem können Unterschiede nach sozialem Status im Verhalten bei der Gesundheitsvorsorge (z.B. Cabeza et al. 2007; Dimitrakaki et al. 2009; MartínLópez et al. 2010) - und damit in Verbindung stehend beim Gesundheitswissen (Sørensen et al. 2015; van der Heide et al. 2013) - festgestellt werden. Die Korrelation von niedrigem Status und schlechtem Gesundheitsverhalten lässt sich nicht für den Alkoholkonsum bestätigen. Studien weisen eher auf den umgekehrten Zusammenhang hin (Schneider/Schneider 2009a; Schneider/ 
Schneider 2009b; Aro/Avendano/Mackenbach 2005; Moore et al. 1999). Untersuchungen zeigen, dass mit höherer Bildung und höherem Einkommen der Anteil der Personen, die überhaupt Alkohol trinken, ansteigt. Ein hoher Alkoholkonsum ist jedoch bei niedrigerem Einkommen und geringerer Bildung häufiger (Dawson et al. 1995; Bloomfield et al. 2006).

Insgesamt zeigen die Studien, dass risikoreiches Verhalten mit höherem sozioökonomischem Status geringer wird. In vielen Studien wird ein sozialer Gradient für gesundheitsrelevantes Verhalten berichtet (Schneider/Schneider 2009a; Pulkki et al. 2003; Kuntz et al. 2016; Lampert/Kroll 2006), also nicht nur ein Unterschied zwischen niedrigstem und höchstem Status, sondern ein Zusammenhang über alle Statusgruppen hinweg. Als Erklärungsmechanismen können zwei Ansätze unterschieden werden. Erstens, die „individualisierte“ Erklärung für Verhalten. Die Verbindung zwischen sozialem Status und Gesundheitsverhalten besteht hierbei aus persönlichen Eigenschaften (Mackenbach 2010; Pulkki et al. 2003). Bisherige Forschung hat gezeigt, dass bestimmte Eigenschaften in niedrigeren sozialen Schichten eher vorhanden sind. In einer US-amerikanischen Studie wurden die „Big Five“ Persönlichkeitseigenschaften (Goldberg 1993) untersucht, wobei in niedrigeren Schichten ein höheres Niveau an Neurotizismus und Verträglichkeit sowie geringe Extraversion, Offenheit und Gewissenhaftigkeit festgestellt wurde (Chapman et al. 2010). Weitere Merkmale, die sowohl mit dem sozialen Status als auch mit Gesundheit und Gesundheitsverhalten in Zusammenhang stehen, sind kognitive Fähigkeiten, Anpassungsfähigkeit und Belastbarkeit (Mackenbach 2010; Bartley 2017: 75). Als Mechanismen, die hinter diesem Zusammenhang stehen, werden vor allem Bedingungen in der Kindheit, insbesondere der Einfluss des Umfeldes (v.a. der Eltern) auf die Sozialisation, und Selektion in der sozialen Mobilität vermutet (Mackenbach 2010). So ist eine zugrundeliegende, aber selten explizit getestete, Erklärung, dass Personen mit geringen kognitiven Fähigkeiten, geringer Anpassungsfähigkeit und niedriger Belastbarkeit in der Schule nicht erfolgreich genug sind um gute, d.h. nicht gesundheitsschädliche und gut bezahlte, Arbeitsplätze zu erhalten. Außerdem wird vermutet, dass ein Mangel dieser Eigenschaften zu risikoreicherem Verhalten führt, da die Botschaften der Gesundheitsbildung nicht (ausreichend) verstanden werden oder die nötige Selbstdisziplin, um diese umzusetzen, nicht vorhanden ist, da eher der kurzfristige Nutzen dem langfristigen Nutzen vorgezogen wird (Bartley 2017: 75 f.). Empirische Belege dafür, dass die Botschaften von Gesundheitserziehung in unteren sozialen Schichten weniger begriffen werden gibt es allerdings nur wenige (ebd.: 77).

Der zweite Ansatz zur Erklärung von unterschiedlichem Gesundheitsverhaltens bezieht sich auf die Kultur in den sozialen Schichten. Normen unterscheiden sich zwischen verschiedenen sozialen Gruppen. Von Bedeutung für das gesundheitsrelevante Verhalten sind bspw. der Stellenwert von gesunder Ernährung, die Bedeutung von Sport und die Akzeptanz von Alkohol-, Tabak- 
oder Drogenkonsum. Aufgrund dieser Einstellungen können sich Verhaltensweisen zwischen sozialen Schichten unterscheiden. Lebensstile werden zudem auch als Abgrenzung zu anderen Gruppen oder sozialen Schichten betrachtet. Bourdieu (1987) beschreibt in der bekannten Studie „Die feinen Unterschiede“, dass der individuelle Geschmack stark von der Gesellschaft geprägt wird. Bei der Kunst und Musik, der Wahl der Kleidung und Freizeitgestaltung und auch beim Essen und Trinken gibt es demnach typische Geschmäcker der unterschiedlichen Schichten, womit eine Abgrenzung verbunden ist. Auch gesundheitsrelevante Verhaltensweisen sind Teil des Lebensstils, durch den sich eine Schicht definieren und abgrenzen kann. Auch wenn sich in heutigen (westlichen) Gesellschaften kaum noch homogene soziale Schichten definieren lassen und eher von Milieus und sozialen Lagen die Rede ist (vgl. Burzan 2011), so ist doch zu erkennen, dass Trends mit soziodemographischen Merkmalen korrelieren. In Deutschland ist beispielsweise eine vegetarische Ernährung sowie der Kauf von Bio-Lebensmittel vor allem bei Personen höherer Bildung verbreitet (Cordts et al. 2013; Masson/Leßmann 2016).

\subsubsection{Psychosoziale Erklärung}

Das psychosoziale Modell erklärt gesundheitliche Ungleichheit durch den Einfluss von psychologischen Risikofaktoren, die im sozialen Leben auftreten. Die zentrale Rolle spielt hierbei Stress, wobei dieser unterschiedlichen Ursachen haben kann. Die bisherige Forschung konzentriert sich vor allem auf soziale Unterstützung, Kontrolle und Anforderungen am Arbeitsplatz (Anforderungs-Kontroll-Modell), das Gleichgewicht von Verausgabung und Belohnung am Arbeitsplatz (Modell sozialer Gratifikationskrisen) sowie den sozialen Vergleich (Hemingway/Marmot 1999). Dieser Erklärungsansatz behandelt auch die Frage, wie soziale Ungleichheiten wahrgenommen werden und wie diese Gefühle im Körper wirken.

Um zu erklären wie biologische Prozesse im Körper durch äußere Gefahren und Stress beeinflusst werden, werden vor allem zwei Reaktionen des Körpers benannt (Brunner 1997; McEwen 1998). Am bekanntesten ist die ,fight-orflight"-Reaktion, durch die der Körper auf Aktivität - Kampf oder Flucht vorbereitet wird. Durch Stress reagiert das Hormonsystem (im sog. sympathoadrenalen System) u.a. mit der Ausschüttung von Adrenalin und Noradrenalin, dadurch gelangt auch Fibrinogen ins Blut, ein Protein, das an der Blutgerinnung beteiligt ist. Zusammen mit der Verengung der Kapillargefäße wird dadurch ein großer Blutverlust von einer Wunde verhindert (Bartley 2017: 91). Gleichzeitig steigen der Puls und der Blutdruck. Die zweite Reaktion des Körpers auf Stress passiert durch die Hypothalamus-Hypophysen-Nebennierenrinden-Achse (HPA-Achse), ein komplexer Hormonregelkreis. In Stresssituationen wird über diesen Mechanismus u.a. Cortisol ausgeschüttet (Johnson et al. 
1992), was zu einer Erhöhung der Energie führt, die der Körper aufwenden kann. Dies geschieht durch die Mobilisierung der Fett- und Zuckerreserven im Blut. In Situationen, die heutzutage Angst, Wut oder Stress auslösen, ist oft keine physische Aktivität als sofortige Reaktion nötig oder möglich - beliebte Beispiele sind Verkehrsstaus oder Mobbing bei der Arbeit. Daher werden die zur Verfügung gestellten Energiereserven nicht verbrannt. Es sammeln sich Rückstände von Fett und Zucker im Blut an, wodurch sich die Blutgefäße verengen. Das erhöhte Level an Fibrinogen fördert dann die Klumpenbildung im Blut, was zu einem Herzinfarkt führen kann. (Bartley 2017: 92). Cortisol ist zudem relevant für das Immunsystem, u.a. besitzt es immunsuppressive Eigenschaften, d.h. dass es die normalen Reaktionen des Immunsystems unterdrücken kann (Schubert 1998). Diese körperlichen Reaktionen, wie z.B. das ausgeschüttete Adrenalin, sind kurzzeitige Reaktionen. Sobald die Stresssituation überstanden ist, kehrt der Körper zu den normalen Abläufen zurück. Ist ein Entkommen aus der Situation nicht möglich, dann wird sie zum chronischen Stress (vgl. Gilbert 2006: 288). Vor allem anhaltender Stress kann negative gesundheitliche Folgen haben (McEwen 1998). Beispielsweise kann durch Schwankungen im Blutdruck bei anfälligen Personen ein Herzinfarkt ausgelöst werden (Muller/Tofler/Stone 1989). Eine Metaanalyse bestätig, dass Stress mit einer erhöhten Wahrscheinlichkeit an Herzerkrankungen zu leiden verknüpft ist und emotionale Stresssituationen kardiovaskuläre Ereignisse (Herzinfarkt, Schlaganfall, kardiovaskulärer Tod) auslösen können (Steptoe/Kivimäki 2013). Die beschriebenen Reaktionen des Körpers werden nicht nur bei physischer Gefahr ausgelöst, sondern auch durch viele andere Arten einer Bedrohung und Stress (McEwen 1998). Auch alltäglicher Stress an sich und der Umgang mit Stress kann langfristige gesundheitliche Folgen haben (Leger/ Charles/Almeida 2018). Dazu zählt auch die Belastung durch finanzielle Probleme, die in Studien in Zusammenhang mit verschiedenen Reaktionen des Körpers gebracht werden konnte, z.B. mit oxidativem Stress und erhöhten Entzündungswerten im Blut (Cutrona et al. 2015; Palta et al. 2015), und eine psychologische Belastung darstellt (Gathergood 2012; Drentea/Reynolds 2015).

Eine wichtige Rolle für Stress spielen die Bedingungen am Arbeitsplatz. Dem Anforderungs-Kontroll-Modell (Theorell/Karasek 1996) zufolge besteht Stress in besonderem Maße bei der Ausübung von Arbeiten, die durch hohe (quantitativen) Anforderungen und geringe Autonomie gekennzeichnet sind. Diese Merkmale sind vor allem bei niedriger beruflicher Position zu erwarten. Typische Beispiele für solche Tätigkeiten sind Fließbandarbeit und Reinigungsdienste. Eine schlechtere Gesundheit von Personen mit geringem sozialem Status könnte somit auf die höhere Stressbelastung bei der Arbeit zurückzuführen sein. Einige, aber nicht alle, Studien weisen darauf hin, dass Arbeiten mit hoher Anforderung und geringer Kontrolle u.a. mit hohem Blutdruck, Herzerkrankungen und Depression in Verbindung stehen (Siegrist 2005: 73; Bartley 2017: 97). Andere Merkmale der Erwerbsarbeit betrachtet das Modell 
sozialer Gratifikationskrisen. Eine Gratifikationskrise besteht, wenn die Belohnung (Lohn, Wertschätzung, Aufstiegsmöglichkeiten, Sicherheit des Arbeitsplatzes) nicht der Verausgabung (Anforderungen, Verpflichtungen) entspricht (Siegrist 1996). Studien belegen, dass die Kombination von hohem Aufwand und geringer Belohnung mit Herz-Kreislauf-Erkrankungen (Siegrist 1995; Siegrist/Peter 1996; Tsutsumi/Kawakami 2004), der Sterblichkeit an Herz-Kreislauf-Krankheiten (Kivimäki et al. 2002), Typ 2 Diabetes (Kumari/Head/Marmot 2004), Depression (Tsutsumi et al. 2001) und Alkoholabhängigkeit (Head 2004) verbunden ist.

Ein weiterer Faktor, der oft zusammen mit anderen Arten von Stress untersucht wird ist sozialer Rückhalt (,social support"). Es können verschiedene Formen des Rückhalts unterschieden werden: emotionaler Rückhalt (Wertschätzung, Zuneigung, Vertrauen, Interesse, Zuwendung), Rückhalt durch Anerkennung (Bestätigung, Feedback, positiver sozialer Vergleich), Rückhalt durch Information (Rat, Vorschläge, Handlungsanweisungen, geteiltes Wissen) und instrumentaler Rückhalt (Hilfe durch zeitliche Präsenz, Arbeit/Mitarbeit, finanzielle Mittel) (House 1981; Siegrist 2005: 74). Der Einfluss auf die Gesundheit kann dabei über zwei Mechanismen wirken. Einerseits ist fehlender sozialer Rückhalt ein eigener Risikofaktor, andererseits wirkt sozialer Rückhalt als Puffer bei Stresserfahrungen (Siegrist 2005: 74). Eine Bestätigung dieser Theorie liefern vor allem Studien, die Mortalität analysieren (HoltLunstad/Smith/Layton 2010). Teilweise widersprüchliche Ergebnisse liefern hingegen Untersuchungen, die sozialen Rückhalt im Zusammenhang mit der Erkrankung an Herzkrankheiten und Schlaganfall untersuchen, wobei allerdings ziemlich deutlich belegt ist, dass sozialer Rückhalt für die Genesung von diesen Erkrankungen eine positive Wirkung hat (Berkman/Krishna 2014; Kiecolt-Glaser et al. 2005). Manche Studien zeigen zudem, dass Personen mit höherer sozialer Position eher sozialen Rückhalt erhalten (Brandt 2009; Matthews/Stansfeld/Power 1999; Power/Matthews 1997), was eine Teilerklärung für den Zusammenhang von Status und Gesundheit sein könnte. Allerdings existieren auch Forschungsergebnisse, die gegen diese These sprechen (Kalmijn/Saraceno 2008; Deindl/Brandt 2015).

Vielfach belegt ist die Tatsache, dass psychologische Stressoren die HPAAchse aktivieren und somit den Cortisolspiegel beeinflussen können. Dieser Effekt ist jedoch nicht gleich stark bei allen Arten von Stressfaktoren. In einer Metaanalyse haben Dickerson und Kemeny (2004) 208 Studien untersucht, in denen Experimente durchgeführt wurden um den Zusammenhang von psychologischer Belastung und der Ausschüttung von Cortisol zu erforschen. Dabei mussten die Probanden Aufgaben ausführen oder waren Situationen ausgesetzt, die Stress verursachen. Davor und danach wurde der Cortisolspiegel gemessen. Das Ergebnis der Metaanalyse zeigt, dass ein stärkerer Anstieg des Cortisolspiegels gemessen wurde, wenn die Aufgaben mit einer Bewertung von anderen verbunden waren, z.B. in Situationen, in denen ein Vergleich mit 
anderen Teilnehmern möglich war oder ein bewertendes Publikum anwesend war. Am stärksten war die Ausschüttung von Cortisol, wenn eine Beurteilung durch andere mit einer Aufgabe kombiniert war, bei der die Probanden ein Scheitern nicht verhindern konnten (eine „unkontrollierbare“ Situation z.B. durch eine negative Bewertung unabhängig der Leistung oder einem absichtlich zu hohen Schwierigkeitsgrad der Aufgabe). Dieses Ergebnis unterstützt somit die Theorien, die davon ausgehen, dass der soziale Vergleich und Erwartungen von Anderen Stress verursachen können. Insbesondere wenn man selbst in dem Vergleich schlecht abschneidet oder die Erwartungen nicht erfüllen kann, sind negative Folgen möglich (siehe auch die Ausführungen zum relativen Einkommen in Kapitel 2.2.6 sowie die ,social rank“ Theorie in Kapitel 2.3.2). Dieser Theorie zufolge geht eine niedrige soziale Position mit ständigen Niederlagen im gesellschaftlichen Vergleich und somit mit chronischem Stress einher (vgl. Präg/Mills/Wittek 2014).

\subsubsection{Materialistische Erklärung}

Ein weiterer Ansatz, um die bestehende gesundheitliche Ungleichheit zwischen sozialen Schichten zu erklären, konzentriert sich auf materielle Ursachen. In der Tat wird in vielen Studien ein Gradient im Zusammenhang von Einkommen mit Gesundheit und Mortalität beobachtet (z.B. Huijts/Eikemo/Skalická 2010; Marmot et al. 1997; Waldron 2013; Lampert/Kroll 2006; Präg/Mills/Wittek 2014). Es gibt wohl keine plausible, direkte kausale Erklärung dafür, warum über alle sozialen Schichten hinweg ein höheres Einkommen die Lebenserwartung erhöht (Bartley 2017: 105). Vielversprechender ist es, sich anzuschauen, in welcher Weise Einkommen als Ursache und Indikator von gesundheitlichen Risikofaktoren in Verbindung steht. Aber auch mit dieser Erklärung ist ein rein materieller Ansatz für die heutigen westlichen Industrieländer schwer vorstellbar. Kälte und Feuchtigkeit in der Wohnung sowie zu wenig Kalorien und Nährstoffe in Mahlzeiten dürfte heutzutage nur in wenigen Fällen (in den ganz unteren Einkommensschicht, v.a. bei Obdachlosigkeit) ein Problem sein und erklären somit nicht den Gradienten (vgl. ebd.: 109).

Ein aussichtsreicherer Ansatz ist hingegen die Betrachtung des Wohnumfelds und dessen gesundheitsrelevante Merkmale (vgl. Chum 2012; Sass et al. 2017; Schneider/Mohnen 2016). Die Mehrzahl der Studien, die den Zusammenhang von Einkommen und Umweltbelastungen in der Wohngegend untersuchen, stammt aus den USA. Dabei wird oft von größeren Belastungen in den Gegenden berichtet, in denen vor allem untere Schichten wohnen (Diekmann und Meyer 2010). Hierbei ist jedoch anzumerken, dass die Segregation nach sozialem Status in den USA deutlich stärker vorhanden ist als in den meisten Europäischen Ländern (Musterd 2016: 332). Aber auch in Europa gilt, dass Personen mit besserer finanzieller Situation, gerade im Falle lokal begrenzter 
Umweltbelastungen [...] über die Exit-Option der Abwanderung verfügen und in bevorzugte Wohngebiete mit höheren Grundstückspreisen und Mieten ausweichen" (Kraemer 2007: 352f.). Studien zu Luftschadstoffen kommen zu dem Ergebnis, dass mit höherem sozialem Status eine im Durchschnitt geringere Belastung der Luft mit Schadstoffen, z.B. mit geringerer Stickstoffdioxidkonzentration, einhergeht (Überblick bei Bolte/Kohlhuber 2009). Diekmann und Meyer (2010) beschreiben anhand von objektiven Messdaten einen sehr schwachen negativen Zusammenhang von Einkommen und Lärmbelastungen in den Schweizer Städten Basel und Bern. Eine andere Studie über die Mietpreise in Genf zeigt auf, dass die Mieten sinken je stärker die Lärmbelastung ist (Baranzini/Ramirez 2016). Auch in einer schwedischen Studie wird ein Zusammenhang von Immobilienpreisen und Verkehrslärm aufgezeigt (Wilhelmsson 2000). Der Zusammenhang von geringem Einkommen und größeren Umweltbelastungen kann somit auch für europäische Länder nicht ganz ausgeschlossen werden.

Ein weiterer Risikofaktor, der mit geringem Einkommen verknüpft ist, ist eine niedrige berufliche Position und Qualifikation, die oft mit schlechten oder gefährlichen Arbeitsbedingungen und hoher Arbeitsbelastung einhergeht (Lampert et al. 2017; Dragano 2016: 174; Bartley 2017: 111; Bosch 2012). Im Gegensatz zum Zusammenhang von Einkommen und Wohnsituation ist hier ein geringes Einkommen nicht die Ursache von schlechten oder gefährlichen Arbeitsbedingungen. Welche beruflichen Positionen erreicht werden können und das Risiko von Arbeitslosigkeit hängen hingegen stark von der Bildung ab (Müller/Brauns/Steinmann 2002; Reinberg/Hummel 2007).

Die gesundheitlichen Vorteile, die man durch Geld erhalten kann, betreffen nicht nur die zum Überleben notwendigen Bedürfnisse (Nahrung und Wohnung) und eine potentiell gesündere Lebensweise (Medizinische Versorgung, Medikamente, Pflege). Auch die Kosten für die soziale Partizipation spielen eine Rolle (Bartley 2017: 113). Soziale Beziehungen, Anerkennung und Zugehörigkeit zur Gesellschaft sind für die Gesundheit und das Wohlbefinden von großer Bedeutung (vgl. Arezzo/Giudici 2017; Holt-Lunstad/Smith/Layton 2010). Morris et al. (2007; sowie Morris 2000) betrachten in ihrer Studie über die „Kosten eines gesunden Lebens“ daher neben den Kosten für Nahrungsmittel, Miete und Heizung auch die Ausgaben für Kleidung, Körperpflege, Fortbewegung, sowie geringe Beträge für Bücher, Telefon, Fernsehen, auswärts essen gehen sowie Sparbeträge für einen Urlaub und Rentenbeiträge. Auch wenn auf diese Weise ein Geldbetrag ermittelt wird, der theoretisch für ein gesundes Leben notwendig ist, ist nicht sichergestellt, dass Personen mit diesem verfügbaren Einkommen auch ein gesundes Leben führen. Für was Menschen ihr Geld ausgeben und an welcher Stelle sie sparen ist sehr unterschiedlich (Hagemann 1998: 34ff.). Ein Verhalten, das jedoch immer wieder bei Geldknappheit beschrieben wird, ist, dass Eltern ihre Bedürfnisse und Wünsche hinten anstellen, um die Bedürfnisse der Kinder zu befriedigen 
(Wendt 2010: 154 ff.). Nicht immer werden die biologischen Bedürfnisse zuerst gestillt, soziale und hedonistische Bedürfnisse sind oftmals genauso wichtig. In der Studie von Wendt „,ällt auf, dass die Befragten [bei einer Verschlechterung der finanziellen Situation] im Grunde in fast allen Konsumbereichen (auch bei Kleidung und Nahrung) zu Einschränkungen bereit sind, auf ihre ,Vergnügungen" aber nur ungern verzichten“ (ebd.: 157). In dieser Aussage wird deutlich, dass Nahrung und eine warme Wohnung alleine für ein gesundes Leben nicht ausreicht. Auch die Partizipation am sozialen Leben ist wichtig, und - vor allem bei geringem Einkommen - zeigt sich: ,psychological and social survival compete with biological need" (Bartley 2017: 116). Das zeigt sich z.B. auch daran, dass manche Eltern ihren Kindern trotz finanziellen Problemen teure Markenkleidung kaufen, um die Kinder vor Ausgrenzung zu schützen (Wendt 2010: 156).

\subsubsection{Selektionshypothese}

In den vorangegangenen Abschnitten wurden Mechanismen beschrieben, wie der soziökonomische Status auf die Gesundheit wirken kann. Neben dieser kausalen Erklärung ist auch die umgekehrte Wirkrichtung denkbar, wobei der Status durch die Gesundheit bestimmt wird (siehe Glymour/Avendano/Kawachi 2014). Diese Selektionshypothese geht davon aus, dass gesunde Personen auf dem Arbeitsmarkt bessere Chancen haben eine hohe Position zu erreichen und somit die Ungesunden in einen niedrigen sozialen Status selektiert werden. Dieser Mechanismus ist insbesondere in der Jugend von Bedeutung, da hier der Grundstein für die berufliche Laufbahn gelegt wird. Doch auch in höherem Alter können Selektionsprozesse auftreten. Die gesundheitliche Situation einer Person kann Auswirkungen auf das Einkommen haben, das durch Erwerbsarbeit generiert wird (vgl. Porru/Burdorf/Robroek 2018). Im schlimmsten Fall droht der komplette Wegfall des Erwerbseinkommens durch Arbeitsunfähigkeit. Und so ist es denkbar, dass sich die durchschnittlich schlechtere Gesundheit von Personen mit geringem Einkommen zumindest teilweise darauf zurückführen lässt, dass diese Gruppe zum Teil aus Personen besteht, die aufgrund ihrer Gesundheitssituation ein geringes Einkommen haben. Dabei ist zu bedenken, dass finanzielle Ressourcen und Gesundheit in einem gegenseitigen Wechselverhältnis stehen und sich über den gesamten Lebenslauf beeinflussen können (siehe Kapitel 2.2.5).

Auf die Frage, welcher Mechanismus - kausale Wirkung oder Selektion der vorherrschende ist, kann keine klare Antwort gegeben werden. Von den 34 Studien, die in einer Metaanalyse untersucht wurden, deuten zwölf Studien auf einen kausalen Zusammenhang hin, zehn Studien deuten auf Selektion hin und die restlichen unterstützen beide Hypothesen gleichermaßen (Kröger/ Pakpahan/Hoffmann 2015). Diese Untersuchung stellt jedoch fest, dass die 
Messung des sozioökonomischen Status eine Rolle spielt. Wurden Indikatoren verwendet, die eng mit dem Arbeitsmarkt verknüpft sind, dann wird sowohl die Kausal-, als auch die Selektionshypothese unterstützt. Bei Verwendung von Bildung und Einkommen als Maß für den sozioökonomischen Status sprechen die Ergebnisse hingegen eher zugunsten der kausalen Erklärung (ebd.). Eine neuere Analyse von europäischen Lebensläufen beschreibt, dass die beiden Mechanismen je nach Alter unterschiedlich stark wirken. Demnach tritt im jungen Erwachsenenalter (Übergang von Kindheit zu Erwerbsleben) sowohl der kausale Effekt, als auch der Selektionseffekt auf, im höheren Alter (beim Übergang vom Erwerbsleben in die Rente) wirkt jedoch hauptsächlich der kausale Effekt (Hoffmann/Kröger/Pakpahan 2018a).

Eine weitere mögliche Erklärung für die beobachtete Korrelation von sozioökonomischem Status und Gesundheit ist die ,indirekte Selektion“ (Marmot et al. 1997). Sie beschreibt wie dritte (unbeobachtete) Eigenschaften (,confounder") sowohl den sozioökonomischen Status als auch die Gesundheit beeinflussen und so eine Scheinkorrelation entsteht. Hierbei kommen z.B. Persönlichkeitsmerkmale, Präferenzen im Verhalten und Lebensstil oder der familiäre Hintergrund infrage (Glymour/Avendano/Kawachi 2014; Hoffmann/ Kröger/Pakpahan 2018b). Die Bedeutung der indirekten Selektion für den Zusammenhang von sozioökonomischem Status und Gesundheit ist umstritten, mal wird ihr mehr, mal weniger Anteil an den beobachteten Zusammenhängen zugeschrieben (vgl. Elstad 2010; Blane/Smith/Bartley 1993; Dragano/Siegrist 2006; Kawachi/Adler/Dow 2010).

\subsubsection{Lebenslaufperspektive}

Betrachtet man den Zusammenhang von sozialem Status und Gesundheit, dann ist es angebracht, nicht nur den aktuellen Status, sondern auch die Vergangenheit zu betrachten, im Idealfall den ganzen Lebenslauf. Mit dieser Lebenslaufperspektive können langfristige Effekte von Benachteiligungen oder Lebensereignissen, egal wie lange diese zurückliegen, in die Analyse integriert werden. Es existieren bereits viele Studien, die langfristige Effekte aufzeigen, z.B. dass ein höherer sozialer Status in der Kindheit mit besserer Gesundheit im höheren Alter und geringerer Mortalität verknüpft ist (Brandt/Deindl/Hank 2012; Galobardes/Lynch/Smith 2008). Auch Zusammenhänge von Kindheitsbedingungen mit der mentalen Gesundheit konnten in Studien nachgewiesen werden (Provençal/Binder 2015; Angelini/Howdon/Mierau 2018).

Ein Erklärungsmodell für solche langfristigen Folgen bietet das Modell der kritischen Perioden (Kuh 2003). Es beruht auf der Annahme, dass eine Belastung, die in einem bestimmten Alter auftritt, für bestimmte Folgen für die spätere Gesundheit verantwortlich ist. Diese Vorstellung stammt aus der Biologie und bezieht sich hauptsächlich auf die Entwicklung des Fötus während der 
Schwangerschaft. Aber auch im Wachstum von Säugling und in der Kindheit ist der Organismus anfällig für langfristige Schädigungen. Es gibt beispielsweise Zeitabschnitte beim Heranwachsen eines Fötus, in denen sich bestimmte Organe ausbilden. Ist die werdende Mutter einer Belastung ausgesetzt, z.B. Krankheiten, Mangelernährung oder Alkoholkonsum, kann sich dies negativ auf das Wachstum der Organe des Kindes auswirken (vgl. Dragano/Siegrist 2006). Die verzögerte oder gestörte Entwicklung des Fötus kann Folgen haben, die erst viele Jahre später sichtbar werden (vgl. Bock et al. 2015; Provençal/Binder 2015). Daher wird diese Theorie auch als Latenzmodell bezeichnet (Siegrist 2005: 77). Eine abgeschwächte Variante der Idee der kritischen Perioden ist das Modell der sensitiven Perioden. Hierbei hat eine Belastung zu einem bestimmten Zeitpunkt im Lebenslauf nicht zwangsläufig eine entsprechende Folge, jedoch geht sie mit einer höheren Wahrscheinlichkeit, z.B. für eine bestimmte Krankheit einher, als zu anderen Zeitpunkten. Ein Beispiel für eine Belastung in einer sensitiven Periode in der Entwicklung des Gehirns liefert eine Studie, die den Einfluss von regelmäßigem Schlafrhythmus in der Kindheit auf kognitive Fähigkeiten von Kindern untersucht. UnregelmäBige Schlafzeiten in der frühen Kindheit haben einen deutlichen nachteiligen Effekt auf die Ergebnisse der kognitiven Tests. In der späteren Kindheit - im Alter von sieben Jahren - ist der negative Einfluss unregelmäßiger Schlafzeiten viel geringer (Kelly/Kelly/Sacker 2013).

Eine einfache Form der Lebenslaufperspektive ist das Kumulationsmodell (Graham 2002). Es geht davon aus, dass sich die Gesundheit umso mehr verschlechtert, je öfter, intensiver und länger die Belastungen sind, denen eine Person über die gesamte Lebenszeit ausgesetzt ist. Frühere Belastungen verstärken dabei den Effekt von späteren Belastungen. In den meisten empirischen Studien werden Belastungen aufsummiert, unabhängig von der Art der Belastung und vom Zeitpunkt des Auftretens. Doch auch mit dieser simplen Methode zeigen sich Zusammenhänge mit vielen verschiedenen Gesundheitsindikatoren (Pudrovska/Anikputa 2014; Bartley/Plewis 2002; Tabassum et al. 2008).

Dass sich der soziale Status und die Gesundheit gegenseitig beeinflussen ist in besonderem Maße bei der Betrachtung des gesamten Lebenslaufes von Bedeutung. Dies wird im Pfadmodell berücksichtigt, denn es beschreibt den Zusammenhang von sozialem Status und der Gesundheit durch soziale Selektionsprozesse (vgl. Dragano/Siegrist 2006). Wenn beispielsweise die Intelligenz eines Kindes durch frühkindliche oder intrauterine Störungen vermindert oder die Förderung der Eltern gering ist, besteht ein größeres Risiko einen niedrigen Schulabschluss zu erlangen, was wiederum schlechtere Chancen auf dem Arbeitsmarkt zur Folge hat. Daraus kann längere Arbeitslosigkeit oder belastungsreiche Arbeit resultieren, was wiederum die Gesundheit beeinflusst. 
Eine mit dem Alter schlechter werdende Gesundheit wird in der Gesundheitsökonomie mit dem Konzept des Gesundheitskapitals (,stock of health capital") beschrieben (Grossman 1972). Die verfügbare Gesundheit nimmt mit dem Lauf der Zeit ab. Um dem körperlichen Verfall entgegenzuwirken und den Körper gesund zu erhalten muss in das Gesundheitskapital investiert werden. Mit höherem Alter beschleunigt sich der Verfall und höhere Investitionen sind nötig.

Es gibt widersprüchliche Theorien darüber, wie sich die gesundheitlichen Unterschiede zwischen Statusgruppen im Alter entwickeln (vgl. Schaan 2013a: 20ff.). Einerseits könnten die Unterschiede mit zunehmendem Alter größer werden, da sich dem Kumulationsmodell folgend die Benachteiligungen bzw. die Vorteile über den Lebenslauf aufsummieren (Ross/Wu 1996). Andererseits ist auch denkbar, dass die Unterschiede geringer werden, da sich die Personen mit niedrigerem Status an die Stressoren anpassen und so eine Immunität oder Gewöhnung eintritt. Eine weitere mögliche Erklärung ist, dass mit zunehmendem Alter die Stressoren, die auf den sozialen Status zurückzuführen sind, weniger werden. Zudem ist ein Selektionseffekt wahrscheinlich: Aus der Gruppe der Benachteiligten überleben vor allem die Gesunden bis ins höhere Alter, während bei den hohen Statusgruppen auch die „Schwächeren“ wahrscheinlicher überleben. Da in den niedrigeren Statusgruppen die Überlebenden also mit zunehmendem Alter immer selektiver werden sollte der Unterschied in der durchschnittlichen Gesundheit zwischen den Statusgruppen kleiner werden (vgl. Schaan 2013a: 22).

\subsubsection{Der Einfluss von Armut auf Gesundheit}

Die in den vorangegangenen Kapiteln erwähnten Theorien gesundheitlicher Ungleichheit sollen hier mit Fokus auf die Effekte von Einkommen bzw. Armut zusammengefasst werden. Der Zusammenhang von Einkommen und physischer Gesundheit ist am deutlichsten bei Menschen, die in absoluter Armut leben. Wer sich nicht ausreichend Nahrung leisten kann, kann nicht lange gesund bleiben. Auch wenn das Geld nicht für eine warme Wohnung, warme Kleidung oder für eine Versorgung mit Trinkwasser reicht, sind gesundheitliche Konsequenzen zu erwarten (z.B. Gemmell 2001; World Health Organization 2017d). Werden diese Grundbedürfnisse erfüllt, kann man dennoch davon ausgehen, dass das Einkommen für die Gesundheit aus mehreren Gründen eine wichtige Rolle spielt.

Je nach Gesundheitssystem muss die Inanspruchnahme von ärztlichen Leistungen und Medikamenten mehr oder weniger aus eigener Tasche bezahlt werden. Eine europäische Studie zeigt, dass unzureichender Zugang zu einer Krankenversicherung vor allem in ärmeren Ländern mit geringen staatlichen Gesundheitsausgaben und höherer Einkommensungleichheit zu beobachten ist 
(Jürges 2015). Zudem existiert innerhalb der meisten Ländern ein sozialer Gradient im Krankenversicherungsschutz (ebd.). Eine andere Untersuchung belegt, dass vor allem Personen mit geringem (Haushalts-) Einkommen Arztbesuche aus Kostengründen verschieben oder ausfallen lassen (Tur-Sinai/Litwin 2015). Zudem sind finanzielle Ressourcen auch für die Pflege von älteren Menschen notwendig (Laferrère/van den Bosch 2015).

Ein anderer Ansatz ist die Betrachtung des Einkommens als relatives Maß. Demnach ist nicht die absolute Höhe des Einkommens von Bedeutung, sondern der Vergleich zu einer Referenzgruppe. Das Einkommen kann als Indikator für die soziale Position innerhalb der Gesellschaft angesehen werden (Wilkinson/Pickett 2009a; siehe auch Kapitel 2.2.8). Diese ,,relative Deprivationshypothese" beschreibt, dass für Personen, die in der Hierarchie unten stehen, diese Vergleiche zu Stress und Frustration führen (siehe Gravelle/Sutton 2009). Demnach ist eine schlechtere Gesundheit eher durch psychische Mechanismen vermittelt als durch materielle Bedingungen. In einer Studie mit Daten aus Großbritannien untersuchen Wood et al. (2012) wie das absolute und das relative Einkommen mit mentaler Gesundheit zusammenhängen. Als Maß für das relative Einkommen verwenden sie den Rang im Einkommen innerhalb drei unterschiedlicher Vergleichsgruppen, die nach Region, Alter und Bildung gebildet werden. Die Berechnungen zeigen, dass sowohl das Einkommen als auch der Rang im Einkommen (in allen Vergleichsgruppen) mit mentaler Gesundheit verknüpft ist. Werden aber beide Indikatoren gleichzeitig kontrolliert, so kann nur noch für den Einkommensrang einen Effekt festgestellt werden. Diese Ergebnisse sprechen dafür, dass die bestens bekannten Effekte des Einkommens auf die (mentale) Gesundheit über die Position in der Einkommensverteilung und somit über psychologische Mechanismen vermittelt sind. Den gleichen Schluss lässt auch die Untersuchung von Walker et al. (2013) zu, in der der Zusammenhang von Armut und Scham sowie der damit verbundene Umgang der Betroffenen untersucht wird. Durch den Vergleich von Daten aus sieben Ländern, die sich im Niveau des absoluten Einkommens stark unterscheiden (Uganda, Indien, China, Pakistan, Südkorea, Großbritannien und Norwegen) können aufschlussreiche Befunde dargestellt werden. Trotz der großen Unterschiede im absoluten Einkommen und des Lebensstandards sind die Erfahrungen und Wahrnehmungen der in Armut lebenden Personen in allen untersuchten Gebieten sehr ähnlich: Überall ist Armut mit Scham verbunden, die daraus resultiert, dass Betroffene nicht in der Lage sind, ihre eigenen Ziele zu erreichen, die Erwartungen von anderen bzw. der Gesellschaft zu erfüllen sowie dem Gefühl von anderen bewertet zu werden (ebd.: 230). Zu den mit Armut verbundenen Reaktionen gehören Rückzug, Selbsthass, Selbstvorwürfe und gegen sich selbst gerichtete Schuldzuweisungen, woraus Verzweiflung, Depressionen bis hin zu Selbstmordgedanken resultieren können (ebd.). Diese Forschungsergebnisse stimmen überein mit dem Konzept der Niederlage (,defeat") (Taylor et al. 2011). Demnach sind Personen mit 
niedrigem Status ,unterwürfig“ gegenüber Statushöheren und verlieren dabei z.B. an Selbstbewusstsein, was die Entstehung von Depressionen begünstig (siehe Kapitel 2.3.2).

Eine weitere Dimension, neben der Unterscheidung zwischen absolutem und relativem Einkommen, ist der subjektiv empfundene im Vergleich zum objektiven sozialen Status bzw. die empfundene Gerechtigkeit und Angemessenheit des eigenen Einkommens. Verschiedene Studien kamen zu dem Ergebnis, dass der subjektive soziale Status stärker mit der Gesundheit korreliert als der objektiv gemessene Status (Cohen et al. 2008; Schnittker/McLeod 2005; Adler et al. 2000). Diese Ergebnisse stärken die Annahme, dass nicht materielle, sondern psychologische Mechanismen von Bedeutung sind. Zudem gibt es Hinweise darauf, dass ein als ungerecht empfundenes (zu niedriges) Einkommen negative Folgen für die Gesundheit hat (Schunck/Sauer/Valet 2015). Die Erklärung für diesen Zusammenhang erfolgt anhand des Modells der Gratifikationskrisen, bei der ein unangemessener Austausch von Leistung und Belohnung betrachtet wird (siehe Kapitel 2.2.2).

Zentral für die Auswirkungen auf die Gesundheit ist der Stress, der mit der finanziellen Situation verknüpft ist. Der menschliche Körper reagiert auf verschiedene Weisen auf psychische Belastungen (vgl. z.B. Cutrona et al. 2015; Palta et al. 2015), was - vor allem bei langanhaltenden Stresssituationen - ein Auslöser von physischen Krankheiten sein kann. Mit finanziellen Notlagen gehen zudem negative Gefühle wie Zukunftsängste und geringes Selbstbewusstsein einher (Waters/Moore 2002; Prawitz/Kalkowski/Cohart 2013). Dies ist von Bedeutung, da die Verarbeitung von Stress und Belastungen auch langfristig auf die Gesundheit wirken kann (Leger/Charles/Almeida 2018).

\subsubsection{Veränderungen in der finanziellen Situation}

Bisher wurde zwar die Lebenslaufperspektive von Gesundheit erläutert und auch der potentielle Einfluss von Einkommen und Armut dargestellt, dennoch fehlt ein wichtiger Aspekt für die Analyse von Einkommen bzw. Armut im Zeitverlauf: die Folgen von positiven wie negativen Veränderungen in der finanziellen Situation. Veränderungen im sozioökonomischen Status können im Laufe des Lebens in den verschiedenen Dimensionen zustande kommen. Der Erwerbsstatus, das Einkommen und das Vermögen können sich immer wieder ändern. Der Bildungsabschluss verändert sich in der Regel ab einem gewissen Zeitpunkt nicht mehr, jedoch kann gesundheitsrelevantes Wissen in jedem Lebensabschnitt vermittelt werden (siehe z.B. Pignone et al. 2003; Thomson et al. 2003). Es gibt eine Vielzahl an möglichen Ursachen für Veränderungen in der finanziellen Situation. Verschlechterungen entstehen z.B. durch Arbeitslosigkeit oder Renteneintritt (auch des Partners), Wegfall eines Einkommens im Haushalt durch den Tod des Partners oder unvorhergesehene Kosten bspw. 
durch Krankheit, Pflege oder notwendige Reparaturmaßnahmen am Wohneigentum. Verbesserungen in der finanziellen Situation sind bspw. durch eine Gehaltserhöhung, eine Erbschaft oder Auszahlungen von Versicherungen möglich. Die Begleitumstände und Folgen dieser Veränderungen können so vielfältig sein wie die Ursachen und sich daher auf unterschiedliche Arten auf Gesundheit und Gesundheitsverhalten auswirken: Der Tod des Partners kann beispielsweise psychische Belastungen (Trauer), Veränderungen im (Gesundheits-) Verhalten (Ernährung, sportliche Aktivität), weniger soziale Kontakte sowie ein geringeres Einkommen zur Folge haben. Um den Effekt der Einkommensveränderung so gut wie möglich zu identifizieren, sollten diese Ereignisse daher so gut wie möglich in den Berechnungen berücksichtigt werden.

Prinzipiell ist es plausibel, dass eine Verbesserung der ökonomischen Situation einen positiven und eine Verschlechterung einen negativen Effekt auf Gesundheit haben sollte. Vermittelt wird dies vermutlich hauptsächlich über psychologische Mechanismen, z.B. über den sozialen Vergleich und Sorgen um die Zukunft. Ist eine Verschlechterung im Lebensstandard die Folge einer Einkommensveränderung, kann das Wohlbefinden durch Verzicht gewohnter Konsumgüter und Luxusartikel leiden. Denkbar ist auch, dass eine Veränderung in der finanziellen Situation das gesundheitsrelevante Handeln der Betroffenen beeinflusst (Grünheid 2004). Die Studie von Venn und Strazdins (2017) kommt zu dem Ergebnis, dass eine Verschlechterung des Einkommens zu einer Verringerung der körperlichen Aktivität führt. Die Ergebnisse bezüglich gesunder Ernährung sind in dieser Untersuchung uneinheitlich und auch andere Befunde lassen einen Zusammenhang von Einkommen und Ernährungsverhalten unwahrscheinlich erscheinen. Da gesundes Essen nicht unbedingt teurer ist als ungesunde Nahrungsmittel (Lee et al. 2016; Carlson/Frazao 2012; siehe aber auch Jones et al. 2014), dürften die Unterschiede in der Ernährungsweise nach sozialem Status, die in vielen Querschnittstudien aufgezeigt werden, hauptsächlich auf die Bildung und weniger auf das Einkommen zurückzuführen sein (vgl. Irz et al. 2014; Schneider/Schneider 2009a).

Neben den Auswirkungen von Veränderungen in der finanziellen Situation soll in dieser Arbeit auch explizit untersucht werden, ob sich die Effektstärken von Verbesserung und Verschlechterung unterscheiden. Sind die Effekte gleich groß, dann könnte beispielsweise der negative Effekt einer Verringerung im Einkommen durch eine spätere Einkommenserhöhung wieder ausgeglichen werden. Anhaltspunkte, dass sich Effektstärken von Verbesserung und Verschlechterung in der finanziellen Situation unterscheiden, liefert die ,prospect theory", eine ökonomische Theorie zur Erklärung von Entscheidungen in Situationen mit Risiko (Kahneman/Tversky 1979). Ein Teil dieser Theorie beschreibt die Verlustaversion, d.h. dass ein Verlust höher Bewertet wird als ein Gewinn (Tversky/Kahneman 1991; Kahneman/Tversky 1979). So ist bspw. das negative Gefühl bei Verlust von 10 Euro stärker als das positive Gefühl bei einem Gewinn von 10 Euro. Hierbei stimmt die Theorie mit dem „Endowment- 
Effekt" überein, der besagt, dass ein Gut wertvoller eingeschätzt wird, wenn man es besitzt (Thaler 1980). Basierend auf diesen Theorien ist auf die gesundheitlichen Folgen von Einkommensänderungen bezogen daher zu erwarten, dass Verschlechterungen in der finanziellen Situation die (v.a. mentale) Gesundheit stärker negativ beeinflussen als eine Verbesserung in der finanziellen Situation einen positiven Effekt hat. Ein abwechselndes steigendes und sinkendes Einkommen würde dann zu einer auf Dauer schlechteren (mentalen) Gesundheit führen, da die positiven Effekte nicht die negativen Effekte kompensieren könnten.

\subsubsection{Einkommens- und Vermögensungleichheit}

Die heutigen reichen Gesellschaften haben den epidemiologischen Übergang hinter sich. Das bedeutet, dass nicht mehr Infektionserkrankungen, sondern chronische, degenerative und altersbedingte Krankheiten die häufigsten Todesursachen sind (Siegrist 2005: 30). Damit hängt zusammen, dass Gesundheit nicht mehr primär von materiellen Faktoren bestimmt wird (Wilkinson 1996). Die Hypothese über den Effekt der Einkommensungleichheit (, income inequality hypothesis") besagt, dass ab einem bestimmten Niveau an Wohlstand in einem Land der Zusammenhang von absolutem Einkommen (des Landes) und Gesundheit (des Landes) schwächer wird und die Verteilung des Einkommens an Einfluss gewinnt (Wilkinson 1994). Die Hypothese, dass Probleme, die mit niedrigem sozialem Status verbunden sind, in ungleichen Gesellschaften stärker auftreten, wurde bereits in den 1970er Jahren in Studien sowohl über Mordraten als auch Mortalitätsraten überprüft (Pickett/Wilkinson 2015). Die Forschung zum Zusammenhang von Einkommensungleichheit und Gesundheit nahm seit Anfang der 1990er Jahre stark zu (ebd.). Bis zum Ende der 1990er Jahre entwickelte sich die Forschung über Einkommensungleichheit in der Kriminologie unabhängig und unbemerkt von der Gesundheitsforschung (ebd.). Erst seit 2005 beschreiben Wissenschaftler die Zusammenhänge von Einkommensungleichheit mit vielen verschiedenen Indikatoren sozialer Probleme wie bspw. Schwangerschaften von Jugendlichen, Übergewicht, mentaler Gesundheit und Drogenkonsum (Wilkinson/Pickett 2009a; Pickett et al. 2005; Pickett/Mookherjee/Wilkinson 2005; Pickett/James/Wilkinson 2006), wodurch auch die Theorie nicht mehr nur für Gesundheit, sondern allgemeiner formuliert wurde.

In vielen Studien wird betont, dass nicht nur die Verteilung des Einkommens, sondern auch die Ungleichheit im Vermögen im Zusammenhang mit der Gesundheit erforscht werden sollte, um ein genaueres Bild zu erlangen, wie ökonomische Faktoren der Makroebene auf die Individuen wirken. Vermögen unterscheidet sich grundsätzlich von Einkommen. Zum Vermögen zählen Geld- und Sachvermögen, es ist im Lebenslauf stabiler als Einkommen, es 
kann vererbt werden und aufgrund von Schulden negativ sein (Klein 2016: 268; Nowatzki 2012: 404). Vermögen ist eine Quelle von Prestige, sozialem Status, Luxus, Freiheit, finanzieller Sicherheit, Selbstvertrauen sowie von politischer Macht (Nowatzki 2012: 405). Einkommen und Vermögen ist weniger stark korreliert als es intuitiv erscheint, was daran liegen könnte, dass bei großem Vermögen das Einkommen aus Erwerbsarbeit verhältnismäßig gering ist und ein Großteil des Einkommens aus Vermögen resultiert (Skopek et al. 2012). Ebenfalls auf den ersten Blick verwunderlich ist die Tatsache, dass im Durchschnitt das Vermögen in Spanien und Italien größer ist als in Deutschland. Dies ist dadurch zu erklären, dass das Vermögen stark mit der Wohneigentumsquote korreliert und in diesen Ländern, im Gegensatz zu Deutschland, der Anteil an Haushalten, die Wohneigentum besitzen, sehr hoch ist (Deutsche Bundesbank 2013: 31; Kolb/Skopek/Blossfeld 2013). In den meisten Haushalten, vor allem bei geringem Einkommen, stellt das Immobilienvermögen den Großteil des Vermögens dar (Skopek et al. 2012; Deutsche Bundesbank 2016). Empirische Analysen zeigen deutlich, dass Vermögen ungleicher verteilt ist als Einkommen (Skopek/Buchholz/Blossfeld 2011; Klein 2016: 270), u.a. da es im Lebenslauf akkumuliert und über Generationen hinweg weiter gegeben werden kann (vgl. Nowatzki 2012: 405).

Es existiert kaum Forschung zum Zusammenhang von Vermögensungleichheit und Gesundheit. Im Gegensatz dazu ist inzwischen empirisch gut belegt, dass eine hohe Einkommensungleichheit mit schlechterem Gesundheitszustand der Bevölkerung korreliert ist. Dieser Zusammenhang wird in den meisten, aber nicht in allen, Studien anhand unterschiedlicher Gesundheitsindikatoren gefunden (siehe Kapitel 3.3). Es existieren drei Theorien, die für diese Beobachtungen herangezogen werden: Die Erklärung anhand psychosozialer Faktoren, die neomaterialistische Erklärung und eine Erklärung, die den beobachteten Zusammenhang als statistisches Artefakt beschreibt. Diese Theorien wurden anhand der Einkommensungleichheit entwickelt, sie können jedoch auch für die Verteilung des Vermögens angewandt werden.

\section{Psychosoziale Theorie}

Die erste Hypothese, die formuliert wurde, um einen kausalen Zusammenhang von Einkommensungleichheit und Gesundheit zu beschreiben, stammt von Wilkinson (1996). Die Statushierarchie in Gesellschaften ist dieser psychosozialen Theorie zufolge die Ursache des Zusammenhangs von Einkommensungleichheit und Gesundheit (Marmot 2004; Wilkinson/Pickett 2009a). Der psychosoziale Ansatz geht davon aus, dass die Wahrnehmung der eigenen Position in der sozialen Hierarchie u.a. anhand des Einkommens erfolgt. Größere Ungleichheit geht mit größerem Wettbewerb einher, was dazu führt, dass die eigene Position sowie die Position von anderen offensichtlicher wird (vgl. Anderson/Bjorklund/Rambotti 2017: 3). Diese Wahrnehmung und die damit 
zusammenhängenden Vergleichsprozesse können Stress sowie negative Gefühle wie Scham und Misstrauen hervorrufen, welche wiederum in psychoneuro-endokrinen Prozessen im Körper zu schlechterer Gesundheit und stressinduziertem Verhalten wie z.B. Rauchen führen. Große Einkommensungleichheit in einer Gesellschaft bedeutet auch, dass die Stufen der ,sozialen Leiter“ einen großen Abstand haben. Daraus resultieren ein härterer Statuswettbewerb und negativere Beurteilungen des eigenen Status für die meisten Leute (Präg et al 2014). In einer ungleichen Gesellschaft ist man dem chronischen Stress des dauerhaften sozialen Vergleichs ausgesetzt. Auch in dem bedeutenden Werk ,The spirit level“" legen Wilkinson und \& Pickett (2009a) dar, dass Stress für die schlechtere physische Gesundheit und höhere Mortalität in ungleicheren Gesellschaften verantwortlich ist. Als Erklärung für den Zusammenhang von Einkommensungleichheit und mentaler Gesundheit verweisen Sie zudem u.a. darauf, dass in ungleichen Gesellschaften manche Werte, die die Vulnerabilität für mentale Krankheiten erhöhen, eine größere Bedeutung haben. Dazu zählt der hohe Stellenwert von Geld und Besitz sowie eines hohen Ansehens (Wilkinson/Pickett 2009a: 69f; James 2007).

Dass der Vergleich mit Menschen in der direkten Umgebung oder die Bewertung durch andere einen direkten Einfluss auf die Gesundheit haben kann, zeigt das Ergebnis einer Metaanalyse zum Zusammenhang von psychologischer Belastung und der Ausschüttung des Stresshormons Cortisol, der in vielen Experimenten untersucht wurde. Die Metaanalyse zeigt, dass Probanden einen deutlich stärkeren Anstieg des Cortisolspiegels hatten, wenn die Aufgaben, die sie lösen sollten, mit einer Bewertung von anderen verbunden waren, z.B. einem Vergleich mit anderen Teilnehmern oder bei Anwesenheit eines bewertenden Publikums (Dickerson/Kemeny 2004). Dass soziale Vergleiche Stress auslösen können ist also eine gut belegte These. Allerdings wird an der Theorie kritisiert, dass sie über diesen Weg den Gradienten im Zusammenhang von Einkommen und Gesundheit erklären will. Der Theorie zufolge leidet nicht nur die unterste Einkommensschicht unter der Ungleichheit, der Hierarchie und dem Wettbewerb, sondern die Gesundheit der gesamten Bevölkerung wird durch die Verteilung des Einkommens beeinflusst (Wilkinson/Pickett 2009a: 181). Die zweit-privilegierteste Gruppe fühlt sich demnach im Vergleich mit der obersten Gruppe relativ benachteiligt. Vor allem dieser Teil der Theorie wird immer wieder kritisiert: „There is something rather depressing about this idea that not being a ,top dog' in some kind of fixed hierarchy could be so psychologically catastrophic as to have an effect on life expectancy itself" (Bartley 2017: 128).

Bei der Erklärung des Einflusses der sozialen Position auf Gesundheit wird auf Versuche mit nichtmenschlichen Primaten verwiesen, die nahelegen, dass Status mit Stress zusammenhängt (Sapolsky 2005, 2004; siehe auch Kapitel 2.3.2). Weitere Unterstützung erhält die Theorie zudem von Forschungsergebnissen, die zeigen, dass der subjektive Status mit der Gesundheit korreliert 
(Schnittker/McLeod 2005). In einem Experiment, in dem Probanden Erkältungsviren ausgesetzt wurden, konnte ein Zusammenhang von der Erkrankung und subjektivem, jedoch nicht mit dem objektiv gemessenen, sozialen Status festgestellt werden (Cohen et al. 2008).

Ein weiterer Teil der Erklärung ist, dass hohe Unterschiede im Status zwischen den einzelnen Personen und der soziale Vergleich zur Folge haben, dass das Vertrauen in die Mitmenschen, der soziale Zusammenhalt sowie das Sozialkapital geringer werden (Wilkinson 1996; vgl. auch Zak/Knack 2001). Ungleichheit begünstigt zudem Abgrenzung zwischen sozialen Schichten und beeinträchtigt somit das Zugehörigkeitsgefühl der gesamten Gesellschaft (Wilkinson/Pickett 2009a: 51; Kragten/Rözer 2017). Denn der Theorie der Homophilie zufolge vertraut man eher andern Leuten, die einem selbst ähnlich sind (vgl. McPherson/Smith-Lovin/Cook 2001). Ein hohes Maß an Vertrauen ist ein zentraler Faktor für eine Gesellschaft mit geringen sozialen Problemen. Vertrauen und Zuverlässigkeit sind notwendig für Beziehungen, die auf Reziprozität und Kooperation basieren (Wilkinson/Pickett 2017). Eine weitere Argumentation, die auf eine Schwächung des Sozialkapitals abzielt, ist die Überlegung, dass bei großem Wettbewerb das Familienleben und andere Beziehungen vernachlässigt werden (van Deurzen/van Ingen/van Oorschot, Wim J. H. 2015: 479). Ein geringer sozialer Zusammenhalt und geringes Sozialkapital haben wiederum negative Folgen für die Gesundheit (Phongsavan et al. 2006; siehe auch die Ausführung zu sozialer Rückhalt in Kapitel 2.2.2).

Da Studien gezeigt haben, dass die individuelle sozioökonomische Position das Selbstbewusstsein (Twenge/Campbell 2016; Rosenberg/Pearlin 1978) sowie den Optimismus (Heinonen et al. 2006) beeinflusst, sind auch Korrelationen mit der Einkommensungleichheit denkbar. Gesellschaften mit hoher Ungleichheit könnten demnach geringere Level an Selbstvertrauen und Optimismus und somit weniger Ressourcen zur Bewältigung von Stress aufweisen (van Deurzen/van Ingen/van Oorschot, Wim J. H. 2015).

\section{Neomaterialistische Theorie}

Eine andere Theorie, die den Zusammenhang von Ungleichheit und Gesundheit erklären will, argumentiert anhand von Investitionen des Staates in Infrastruktur und öffentliche Güter. Gesellschaften mit geringer Ungleichheit zeichnen sich demnach durch großzügigere Sozialsysteme, höhere staatliche Ausgaben (z.B. für Gesundheit und Verkehr), umfangreichere soziale Sicherung und mehr Investitionen in Humankapital (z.B. Bildung) aus. Diese ,neomateriellen" Faktoren sind in Kombination mit den individuellen Ressourcen für den Zusammenhang von Einkommensungleichheit und Gesundheit verantwortlich (Lynch/Kaplan 1997; Smith 1996). Der Zusammenhang von Investitionen und Ungleichheit in einer Gesellschaft entsteht nicht zufällig: „An une- 
qual income distribution is one result of historical, cultural, and political-economic processes. These processes influence the private resources available to individuals and shape the nature of public infrastructure - education, health services, transportation, environmental controls, availability of food, quality of housing, occupational health regulations - that form the ,neo-material' matrix of contemporary life" (Lynch et al. 2000: 1202).

Kritiker der psychosozialen Hypothese sehen die neomaterielle Hypothese meist als konkurrierende Erklärung an. Wie Rözer \& Volker (2016) darstellen, können die beiden Theorien jedoch durchaus als sich ergänzend angesehen werden. In Ländern mit größerer Ungleichheit sind die staatlichen Ausgaben oft eingeschränkt weil öffentliche Ausgaben Solidarität und sozialen Zusammenhalt erfordert, diese Werte jedoch in den Ländern mit großer Ungleichheit schwächer ausgeprägt sind (Subramanian/Kawachi 2004: 87).

\section{Absolute Einkommenshypothese}

Eine weitere Erklärung für den beobachteten Zusammenhang von Einkommensungleichheit und Gesundheit wurde als Artefakt-Erklärung (Gravelle 1998) oder „absolute Einkommenshypothese“ (Wagstaff/van Doorslaer 2000) bezeichnet. Rodgers (1979) hat theoretisch dargelegt, wie durch einen nichtlinearen Zusammenhang zwischen Einkommen und Gesundheit auf der Individualebene ein Zusammenhang von größerer Einkommensungleichheit und schlechterer Gesundheit auf der Makroebene entstehen kann.

Zur Veranschaulichung wird hier auf das Beispiel und die grafische Darstellung von Gravelle (1998) zurückgegriffen. ${ }^{2}$ Die fettgedruckte Linie in Abbildung 2.1 beschreibt den Zusammenhang von Einkommen $y$ und Mortalitätsrisiko $m$ auf Individualebene: Mit steigendem Einkommen sinkt das Mortalitätsrisiko, wobei der positive Effekt von zusätzlichem Einkommens umso geringer ausfällt, je höher das Einkommen ist. Es sollen zwei Länder verglichen werden, die dasselbe durchschnittliche Einkommen $\bar{y}$, aber eine unterschiedliche Verteilung des Einkommens aufweisen. Zur übersichtlichen Darstellung wird angenommen, in Land A hat die Hälfte der Bevölkerung ein geringes Einkommen $y_{1 A}$ und ein hohes Mortalitätsrisiko $m_{1 A}$. Die andere Hälfte der Bevölkerung hat ein höheres Einkommen $y_{2 A}$ und ein geringeres Mortalitätsrisiko $m_{2 A}$. Das durchschnittliche Mortalitätsrisiko in Land A ist $m_{A}$. In Land B ist die Einkommensungleichheit größer: Die Hälfte der Bevölkerung hat Einkommen $y_{1 B}$ (und Mortalitätsrisiko $m_{1 B}$.), die andere Hälfte hat Einkommen $y_{2 B}$ (und Mortalitätsrisko $m_{2 B}$ ). Obwohl das durchschnittliche Einkommen in beiden Ländern gleich ist, ist das Mortalitätsrisiko in Land B gröBer als in Land A. 
Während in einer älteren Übersicht viele Studien diese Hypothese als mögliche Erklärung dargelegt hatten, widersprechen die meisten neueren Studien der Vermutung, dass die Makrozusammenhänge von Ungleichheit und Gesundheit nur durch dieses statistische Artefakt zustande kommen (Wilkinson/ Pickett 2006, 2009b; Layte/Whelan 2014; Auger et al. 2012).

Abbildung 2.1: Der Effekt von steigender Einkommensungleichheit auf die Mortalität

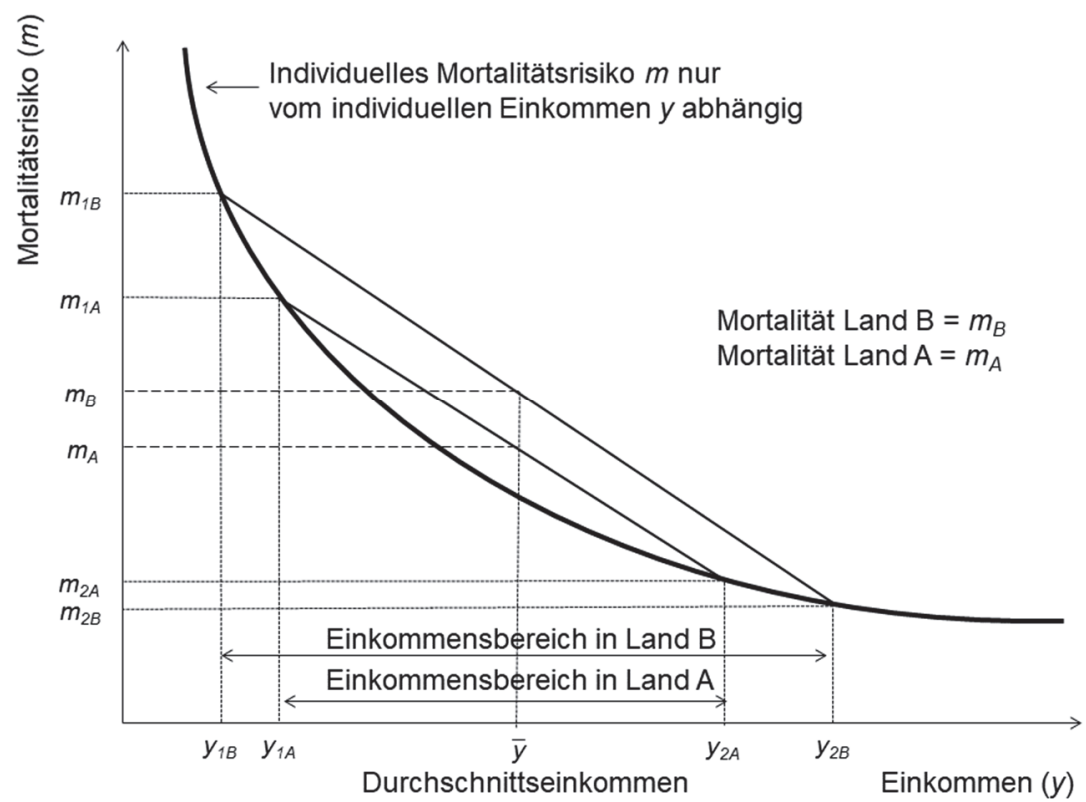

Quelle: Gravelle 1998

Wer leidet unter Ungleichheit?

Es stellt sich die Frage, ob alle oder nur bestimmte Gruppen in einer Gesellschaft von negativen Effekten der Ungleichheit betroffen sind. Dabei haben sich die von Mellor und Milyo (2002) eingeführten Bezeichnungen der „starken“ und „schwachen“ Version der Einkommensungleichheitshypothese verbreitet. Die starke Version beschreibt die Hypothese, dass alle Bevölkerungsschichten von der Ungleichheit beeinflusst werden. Bei der schwachen Version wird hingegen angenommen, dass nur bei der unteren (Einkommens-) Schicht die Auswirkungen von Ungleichheit zu beobachten sind. Bei dieser Frage 
könnte man zuerst an die Personen am unteren Ende in der ökonomischen Rangfolge denken, da diese Gruppe im sozialen Vergleich mit anderen schlecht abschneidet (psychosoziale Hypothese), bzw. die fehlenden staatlichen Investitionen (z.B. im Gesundheitssystem) nicht durch eigene Ressourcen kompensieren kann (neomaterielle Hypothese) (Subramanian/Kawachi 2006). Wilkinson und Pickett sind hingegen der Überzeugung, dass alle Mitglieder einer Gesellschaft von größerer Gleichheit profitieren (Wilkinson/Pickett $2009 b$ ). Begründet wird dies damit, dass geringes Vertrauen und geringer $\mathrm{Zu}-$ sammenhalt in der Gesellschaft für alle sichtbar und erlebbar ist. Eine solidarische und harmonische Gesellschaft ist demnach für das Wohlbefinden aller Mitglieder förderlich, denn von den Problemen die mit großer Ungleichheit einhergehen, wie z.B. Kriminalität, sind alle betroffen. Was den Statuswettbewerb betrifft, so sind alle Personen innerhalb einer Gesellschaft demselben Wettbewerb ausgesetzt. Dass die höheren Schichten auch unter großer Ungleichheit leiden lässt sich damit erklären, dass die Angst vor einem Abrutschen in der Hierarchie und die mögliche Fallhöhe umso größer sind, je ungleicher der Status verteilt ist. Zudem scheinen Menschen die Neigung zu haben sich vor allem mit Bessergestellten zu vergleichen (vgl. van Deurzen/van Ingen/van Oorschot, Wim J. H. 2015: 478; Goerke/Pannenberg 2015; Anderson/Bjorklund/Rambotti 2017: 3). Somit ist der soziale Vergleich auch für relativ hohe Statusgruppen eine schmerzliche Angelegenheit, da es immer jemanden gibt, der noch mehr verdient und einen noch höheren Status hat.

Eine andere theoretische Überlegung beschreibt plausibel einen nach Status unterschiedlichen Effekt von Ungleichheit auf (mentale) Gesundheit. Van Deurzen et al. (2015) gehen davon aus, dass vor allem für die mittlere Schicht negative Effekte auf die Gesundheit auftreten. Personen an der Spitze der Hierarchie haben die Möglichkeit des Vergleichs nach unten, wovon sie profitieren können. Die dadurch entstehenden Gefühle, z.B. Selbstbewusstsein oder Stolz, sind psychologische Ressourcen, die bei der Bewältigung von Stress von Bedeutung sind. Die Personen an dem unteren Ende der Hierarchie sind dieser Theorie zufolge eher weniger zu sozialen Vergleichen geneigt, da sie andere Prioritäten haben, z.B. die finanziellen Probleme im Alltag zu bewältigen. Die Gruppe in der Mitte der Hierarchie hingegen kann sich Vergleiche mit anderen erlauben und hat eher das Ziel Karriere zu machen, wobei höhere Statusgruppen als Vorbild dienen. Da jedoch nur wenige die erwünschte höhere Position erreichen, bleibt die Mehrheit der Mittelschicht unzufrieden mit ihrer Situation (ebd.).

Neben dem sozialen Status gibt es noch zahlreiche andere Merkmale, nach denen man den Effekt von Ungleichheit differenzieren kann. ${ }^{3}$ Diese sind jedoch für diese Studie nicht von Bedeutung.

3 So berichten beispielsweise Torre und Myrskylä (2014) sowie Lynch et al. (2004) von schädlichen Effekten von Einkommensungleichheit vor allem für Kinder und Jugendliche. 
Verknüpfung von psychosozialer und neomateriaistischer Theorie

In der Diskussion um die passende Erklärung für die empirisch aufgezeigten Zusammenhänge zwischen Ungleichheit und Gesundheit gibt es eine Vielzahl an Studien, die mit unterschiedlichen Methoden, zu unterschiedlichen Zeiten, mit unterschiedlichen Gesundheitsindikatoren, in unterschiedlichen Gebieten die Ungleichheit auf unterschiedlich großen geographischen Einheiten untersucht haben (siehe Kapitel 3.3). Die Studie von Chen \& Gotway Crawford (2012) zeigt die Variation der Ergebnisse aufgrund von verschiedenen Gebietseinheiten und Gesundheitsmaßen in den USA. Die Autoren schließen daraus, dass die Ungleichheiten, die auf den verschiedenen geographischen Einheiten gemessen werden, unterschiedlich interpretiert werden können. Dieser Idee folgend schlagen Patel et al. (2018) ein Modell vor, in dem die existierenden Hypothesen den geographischen Ebenen zugeordnet werden (siehe Abbildung 2.2). Auf der individuellen Ebene ist demnach die psychische Belastung der

\section{Abbildung 2.2: Mechanismen des Einflusses von Einkommensungleichheit auf Depression}

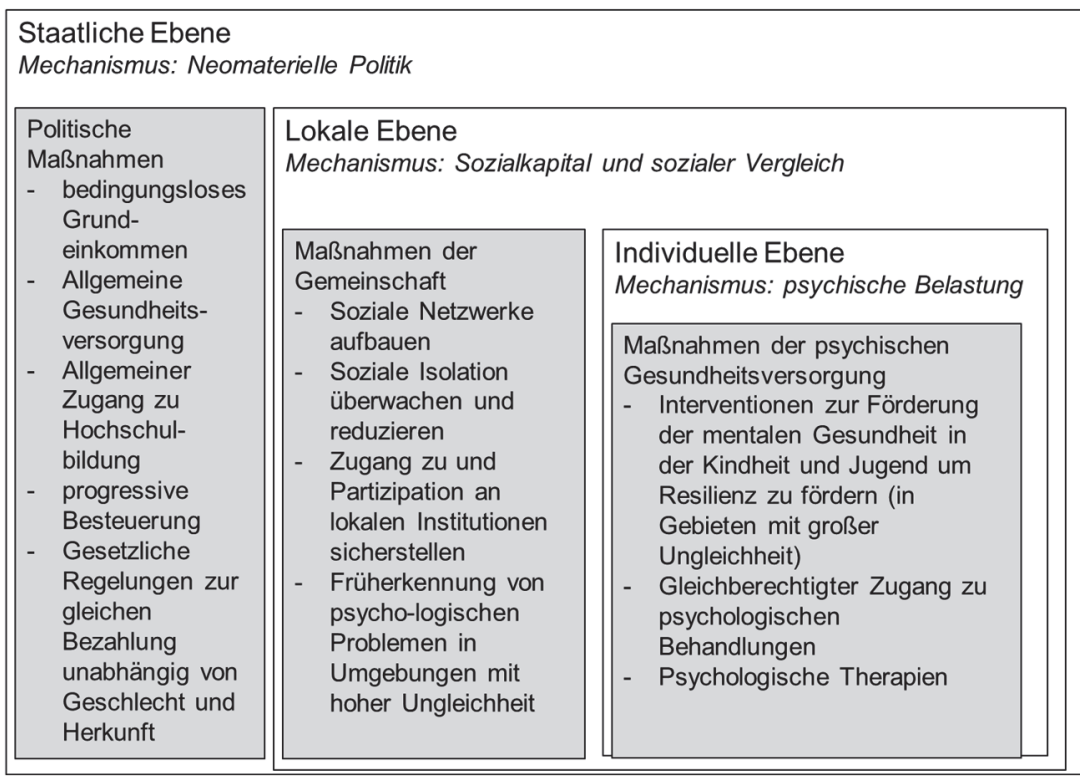

Quelle: basierend auf Patel et al. 2018: 85, eigene Übersetzung. 
ausschlaggebende Mechanismus über den soziale Ungleichheit wirkt. Innerhalb der näheren Umgebung spielen der soziale Vergleich und das Sozialkapital eine wichtige Rolle. Der neomateriellen Hypothese entsprechend sind staatliche Investitionen und die Infrastruktur die entscheidenden Faktoren, die die Ungleichheit einer ganzen Gesellschaft mit der Gesundheit verknüpfen. Dieser Ansatz zeigt, dass sich die psychosoziale und die neomaterielle Theorie vereinen lassen, auch wenn die beiden Erklärungen oftmals als widersprüchlich dargestellt werden. Diese Betrachtungsweise widerspricht Pickett und Wilkinson, die den Mechanismus lediglich auf nationaler bzw. gesellschaftlicher Ebene vermuten (Pickett/Wilkinson 2015: 319).

\subsection{Depression}

Depression ist eine affektive Störung, die gekennzeichnet ist durch Traurigkeit, Verlust von Interessen und Freude, Schulgefühle, geringes Selbstwertgefühl, Schlafprobleme, Appetitlosigkeit, Müdigkeit sowie Konzentrationsschwäche (World Health Organization 2017a). Dabei handelt es sich nicht um übliche Stimmungsschwankungen und die kurzzeitigen emotionalen Reaktionen, die bei alltäglichen Problemen auftreten können. Bei einer Depression sind negative Gedanken und Gefühle stärker und anhaltender (World Health Organization 2017b). Besonders bei einer dauerhaften Depression können alle Bereiche des Lebens beeinträchtigt werden. Mögliche Folgen einer Depression sind u.a. eine geringere Leistungsfähigkeit bei der Arbeit oder in der Schule (Roh et al. 2010; Lerner/Henke 2008), Arbeitsunfähigkeit (Wedegärtner et al. 2011) sowie Rückzug aus sozialen Beziehungen (Lischka 2009; Amann 1991).

Die Erforschung der Depression, die ihren Anfang vor über 2500 Jahren genommen hat, führte im Laufe der Zeit zu vielen verschiedenen Ansätzen zur Erklärung und Heilung der Krankheit und hat zu dem heutigen weitgehenden, aber längst nicht kompletten, Verständnis dieser Krankheit geführt (Balmuth 2017). Eine Unterscheidung von verschiedenen Formen der Depression klassifiziert nach der Ursache: innerhalb des Körpers entstandene Depressionen (endogen), durch anhaltende äußere Umstände und Belastungen durch kritische Lebensereignisse hervorgerufene Depressionen (neurotisch bzw. reaktiv) und als Folge einer körperlichen Erkrankung auftretende Depressionen (somatogen) (Laux 2003; Schaan 2013a: 3). Das heutzutage gängige unitaristische Konzept hingegen unterscheidet nicht nach Ursachen sondern nur nach Schweregrad und Dauer der Depression (Bronisch 1992). Dazu zählt auch der heutzutage vorherrschende biopsychosoziale Ansatz, der davon ausgeht, dass Depressionen meist in Kombination von biologischen, psychologischen und sozialen Faktoren ausgelöst werden. Das „biopsychosocial model of etiology“ (Engel 1977, 1980; Lindau et al. 2003) ist besonders geeignet für die 
Beschreibung der Depression von älteren Menschen, ,because it reminds us that the origins of late-life depression are multiple and range across all three domains" (Blazer/Hybels 2005: 1).

Auch das Vulnerabilitäts-Stress-Modell teilt die Annahme des biopsychosozialen Modells der multikausalen Auslöser von Depression. Es besagt, dass das Auftreten einer Depression durch die Kombination von Stressoren und Vulnerabilität erklärt werden kann (Patten 2013; Scher/Ingram/Segal 2005). Stressoren, also alle äußeren Belastungen wie z.B. negative Ereignisse, ungünstige Lebensumstände und auch chronischer Stress, führen nur bei bereits existierender Vulnerabilität zu einer Depression. Liegt bei einer Person ein Vulnerabilitätsfaktor (z.B. genetische Disposition) vor, so „erhöht sich die Wahrscheinlichkeit, dass bei Exposition gegenüber Stressoren krankheitswertige Stressreaktionen auftreten, im Vergleich zu einer Person ohne diesen Vulnerabilitätsfaktor" (Siegrist 2005: 83). Die Beziehung von Stressoren auf die Stressreaktion bzw. deren Folge, eine Depression, wird also von Vulnerabilitätsfaktoren moderiert. Allerdings gibt es auch Protektivfaktoren, also Faktoren, die gegen eine Entstehung von Depression wirken, z.B. emotionale Unterstützung durch stabile soziale Beziehungen (ebd.).

Dass auch eine frühere depressive Phase ein Vulnerabilitätsfaktor darstellen kann, wird in der Kindling-Hypothese beschrieben (Post 1992). Demnach ist eine (weitere) depressive Phase wahrscheinlicher, je mehr depressive Phasen bereits zuvor aufgetreten sind. Der Theorie zufolge können Stressoren und depressive Phasen neurobiologische Veränderungen im Körper auslösen, wodurch weitere depressive Phasen mehr und mehr unabhängig von äußeren Stressoren werden. Bedeutende Ereignisse oder Situationen im Leben wären somit für die Entstehung der ersten Depression von großer Bedeutung, während wiederholte Depressionen möglicherweise schon durch Kleinigkeiten oder ohne erkennbaren Grund ausgelöst werden (vgl. Lewinsohn et al. 1999; Ormel/Oldehinkel/Brilman 2001; Daley/Hammen/Rao 2000).

In den nächsten Abschnitten werden die wichtigsten Erklärungsansätze für die Entstehung von Depressionen dargestellt. Dabei wird zwischen biologischen, evolutionären, genetischen, psychologischen und sozialen Ursachen unterschieden. Anschließend wird in Kapitel 2.3.6 speziell auf Depressionen im höheren Alter eingegangen. Da die beschriebenen Ursachen von Depression allgemein gehalten sind und ein weites Spektrum - von Genen bis zu Lebensereignissen - abdecken, folgt abschließend in Kapitel 2.3.7 eine Zusammenfassung, die in erster Linie den Zusammenhang von finanziellen Umständen und Depression beschreibt. 


\subsubsection{Biologische Ursachen}

Es gibt verschiedene biologische Faktoren, die als Ursache für Depression in Frage kommen. Eine mögliche Erklärung für Depression ist die mangelhafte Anpassung an Stress (Dickerson/Kemeny 2004). Erklärt wird der Zusammenhang von Stress und Depression demnach durch ein verändertes Hormonsystem bei depressiven Personen, wobei die Funktion der Hypothalamus-Hypophysen-Nebennierenrinden-Achse verändert ist (Burke et al. 2005; siehe auch Kapitel 2.2.2). Dies kann dazu führen, dass bei Stress mehr von dem Stresshormon Cortisol ausgeschüttet wird (Morris/Rao/Garber 2012). Die Ergebnisse einer Meta-Analyse deuten darauf hin, dass depressive und nicht depressive Personen zwar mit ähnlichem Cortisollevel auf einen Stressor reagieren, die depressiven Personen jedoch deutlich länger brauchen um sich davon zu erholen (Burke et al. 2005).

Eine recht neue Entdeckung ist, dass möglicherweise Teile des Immunsystems, die Entzündungen herbeiführen, mit Depression in Verbindung stehen (Slavich/Irwin 2014). Eine Entzündung ist eine Reaktion des Körpers auf physische Verletzungen und Infektionen. Jedoch gibt es Hinweise darauf, dass psychischer Stress die Entzündungsaktivität erhöhen und somit depressive Symptome verursachen kann (Glaser/Kiecolt-Glaser 2005; Anisman/Merali/Hayley 2008).

Eine weitere biologische Erklärung von Depression bezieht sich auf biochemische Prozesse im Gehirn und die dafür notwendigen Neurotransmitter. Diese Botenstoffe sind notwendig um Signale von einer Nervenzelle zur anderen zu übertragen. Auffälligkeiten bezüglich Neurotransmitter wurden bei depressiven Patienten vielfach beobachtet (Anisman/Merali/Hayley 2008). Die Ursache von Depression könnte ein Mangel oder Ungleichgewicht von verschiedenen Neurotransmitter sein (Delgado/Moreno 2000; Nutt 2008). Im Fokus steht hierbei vor allem Serotonin, da dieser Stoff viele Funktionen beeinflusst wie z.B. die Stimmung, den Schlaf, den Appetit, das Hormonsystem, die Körpertemperatur und kognitive Funktionen (Meltzer 1990). Andere Neurotransmitter, die mit Depression in Verbindung stehen sind Noradrenalin, es stimuliert das limbische System, welches einen Einfluss auf die Regulierung von Emotionen hat (Moret/Briley 2011), und Dopamin, es beeinflusst Motivation, Konzentration und Anhedonie (Dunlop/Nemeroff 2007).

In einigen Studien wurden Unterschiede in der Struktur im Gehirn zwischen depressiven und nicht depressiven Personen gefunden. In Untersuchungen mit Magnetresonanztomographie wurden Abweichungen im Volumen von bestimmten Regionen im Gehirn - z.B. Hippocampus, orbitofrontaler Cortex, Putamen und Thalamus - beobachtet (Sexton/Mackay/Ebmeier 2013; Schindler et al. 2018). Ob diese Unterschiede in der Gehirnstruktur Ursache oder Folge einer Depression sind, kann mit diesen Studien jedoch nicht geklärt werden (Videbech/Ravnkilde 2004). 
Ein weiterer Ansatz ist davon abgeleitet, dass eine Komorbidität von Depression u.a. mit Herzkrankheiten, Diabetes und Alzheimer vielfach festgestellt werden konnte (Anderson et al. 2001; Pieper et al. 2008; Härter/Baumeister 2007; Blazer/Hybels 2005). Physische Krankheiten können Auslöser und Folge einer Depression sein (Härter/Baumeister 2007). Die ,vascular depression hypothesis" geht davon aus, dass zerebrovaskuläre Krankheiten die Entstehung von depressiven Symptomen begünstigt (Taylor/Aizenstein/Alexopoulos 2013). Aber auch von anderen Krankheiten wird vermutet, dass sie - vor allem in höherem Alter - Depression verursachen können (Blazer/Hybels 2005; Stoppe 2008). Einschränkungen in der Bewegungsfähigkeit, die auch als Folge von anderen Krankheiten auftreten können, sind ebenfalls eine häufige Ursache von Depressionen im höheren Alter (Hays et al. 1997; Bruce 2001). Zudem können nicht nur die Krankheiten, sondern auch die verwendeten Medikamente gegen somatische Erkrankungen Ursache für das Auftreten depressiver Symptome sein (Härter/Baumeister 2007).

\subsubsection{Evolutionäre Erklärung}

Von der Vielzahl an evolutionären Erklärungen von Depression (siehe Gilbert/Allan 1998: 586; Gilbert 2006) sollen hier die zwei bedeutendsten, die Rangordnungshypothese der Depression ${ }^{4}$ (,social rank theory“) und die Bindungstheorie (,attachment theory“), dargestellt werden. Die Rangordnungshypothese basiert auf der Überlegung, dass Tiere mit ihren Artgenossen im Kampf um Ressourcen sind, z.B. um Gebiete oder Sexualpartner. Erkennt ein Tier, dass es gegen den Widersacher keine Chance hat, dann wendet es eine Rückzugsstrategie an, indem es zeigt, dass es unterwürfig ist. Dadurch kann es unnötige Auseinandersetzungen und Verletzungen vermeiden. Das „,defeat syndrome" beschreibt, dass Tiere mit niedrigem sozialem Rang ihren Artgenossen mit höherem Rang signalisieren, dass von ihnen keine Gefahr ausgeht. Diese Signale werden durch bestimmte Verhaltensweisen gesendet, die auch bei Depression auftreten, z.B. Rückzug, apathisches Verhalten, wenig Appetit, verringertes Sexualverhalten und Hypervigilanz (Gilbert 2006; Gilbert/Allan 1998). Für das unterlegene Tier selbst hat diese „,soziale Niederlage“ negative Folgen wie verringertes soziales Vertrauen (Gilbert/Allan 1998: 587). Es existieren zahlreiche Untersuchungen, die Stressreaktionen bei Tieren in Zusammenhang mit Unterordnung und ,defeat" feststellen (z.B. Vögel (Carere et al.

Diese Übersetzung des hauptsächlich im Englischen verwendeten Begriffs „,social rank theory“ stammt von (Pillmann 2001: 269): „In Ermangelung eines besseren deutschen Ausdrucks soll im Folgenden von der Rangordnungshypothese der Depression die Rede sein“. Eine alternative Übersetzung ist „,soziale Rangtheorie“, die jedoch zu unterscheiden ist von dem Begriff der „Rangtheorie“, der im deutschen für die philosophische „ranking theory“ verwendet wird (siehe Spohn 2012). 
2001), Eidechsen (Greenberg/Chen/Crews 1984), Krebse (Yeh/Fricke/ Edwards 1996)), und auch bei Menschen hängt das Erleben von Niederlagen und Minderwertigkeit mit Depression zusammen (Gilbert/Allan 1998). Zudem gibt es Hinweise darauf, dass eine Niederlage weniger problematisch ist, wenn die Möglichkeit zur Flucht besteht, und im Vergleich dazu größere Auswirkungen hat, wenn man gefangen ist und sich der Situation nicht entziehen kann (,entrapment") (ebd.).

Die andere Theorie, die hier noch Erwähnung finden soll ist die Bindungstheorie (siehe Gilbert 2006). Hierbei wird auf den engen Kontakt zwischen Mutter und Kleinkind verwiesen. Bleibt dieser Kontakt aus, so folgt eine Reaktion des Kindes in Form von Protest und Verzweiflung (,protest-despair reaction"). Zunächst wird mit der Protestreaktion versucht auf sich aufmerksam zu machen und Leid oder eine Notlage zu signalisieren. Gelingt dies nicht, folgt die Verzweiflung: „Despair is thus designed to make an animal stop signaling and moving in the environment (hunker down/ demobilize) when not to do so is dangerous." (ebd.: 290, Hervorhebung im Original). Zu dieser Theorie passen Studien, die zeigen, dass sowohl bei Tieren als auch bei Menschen der Verlust von Beziehungen und nahestehenden Personen oftmals Auslöser einer Depression ist (Kendler et al. 2003; Brown/Harris/Hepworth 1995; Schaan 2013b, siehe auch Kapitel 2.3.5). Damit verknüpft ist auch die Literatur, die aufzeigt, dass die Verfügbarkeit von sozialem Rückhalt die Entstehung von Depressionen beeinflusst. (George et al. 1989; Brown et al. 1986, siehe auch Kapitel 2.2.2).

\subsubsection{Genetische Ursachen}

Wissenschaftliche Studien haben eine genetische Disposition für Depression nachgewiesen. Demnach ist die Wahrscheinlichkeit einer Depression nach kritischen Ereignissen oder bei Belastung durch chronische Stressoren abhängig von genetischen Faktoren (Übersicht bei Lau/Eley 2010). Aber nicht nur Gene, sondern auch deren Verhältnis zur Umwelt und die daraus entstehenden Folgen, werden diskutiert. Die Gen-Umwelt-Korrelation, Gen-Umwelt-Interaktion sowie die Epigenetik sind in diesem Zusammenhang die relevanten Forschungsfelder (vgl. Lesch 2004; Heim/Binder 2012; Lau/Eley 2010). Eine Gen-Umwelt-Korrelation liegt vor, wenn ein Genotyp nicht zufällig verteilt ist, sondern in einer bestimmten Umwelt besonders häufig vorkommt. Genetische Merkmale und Umwelt können korreliert sein, da sich Personen mit einem bestimmten Genotyp in ihre Umwelt hineingeboren werden (passive Gen-Umwelt-Korrelation), sie sich die Umwelt selbst aussuchen (aktive Gen-UmweltKorrelation) oder die Umwelt durch Reaktionen von der sozialen Umgebung beeinflusst wird (reaktive/evozierte Gen-Umwelt-Korrelation). Ein oft genanntes Beispiel sind Kinder, die von ihren Eltern eine hohe Intelligenz geerbt 
haben: Diese Kinder leben in einer Familienumwelt, in der ihre Intelligenz gefördert wird (passiv), durch ihre Intelligenz werden sie auf eine entsprechende Schule geschickt (reaktiv) und sie suchen sich selbst einen Freundeskreis mit ähnlichen Interessen (aktiv). In Bezug auf Depression kann die Gen-UmweltKorrelation einen Beitrag zur Erklärung liefern, warum manche Bevölkerungsgruppen eine höhere Prävalenz haben. Zur Identifikation von kausalen Effekten kann sie jedoch nicht beitragen, sie ist eher ein Hinweis für Selektionsprozesse (Schaan 2013a: 6). Eine Gen-Umwelt-Interaktion liegt vor, wenn die Reaktion auf die Umwelt oder Ereignisse von genetischen Faktoren beeinflusst ist. Insbesondere anhand der Untersuchung von Zwillingen wurde gezeigt, dass die Wahrscheinlichkeit für die Entstehung einer Depression nach bestimmten Lebensereignissen von genetischen Faktoren abhängig ist (für einen Überblick siehe Lau/Eley 2010).

Die Epigenetik ist ein relativ neuer Forschungszweig in der Biologie. Sie untersucht, wie sich die Aktivität von Genen im Laufe des Lebens durch Umwelteinflüsse verändert (für biologische Definitionen siehe Haig 2004 sowie Deans/Maggert 2015). Bildlich gesprochen passiert das dadurch, dass Gene als Reaktion von äußeren Umständen ,aktiviert" oder ,ausgeschaltet“ werden. So haben eineiige Zwillinge zu Beginn des Lebens die genau gleichen Gene, im Alter lassen sich jedoch große Unterschiede feststellen (Fraga et al. 2005). Nachdem zunächst in den meisten epigenetischen Studien physische Krankheiten wie z.B. Krebserkrankungen untersucht wurden, gibt es inzwischen immer mehr Forschung in diesem Feld zu psychischen Störungen (Schaan 2013a: 7).

\subsubsection{Psychologische Erklärungen}

In psychologischen Studien wurde bereits vielfach gezeigt, dass bestimmte Persönlichkeitseigenschaften mit der Wahrscheinlichkeit an Depression zu leiden korrelieren. Depressionen treten demnach häufiger auf bei Personen mit ausgeprägtem Neurotizismus (Uliaszek et al. 2010; Kercher/Rapee/Schniering 2009; Klein/Kotov/Bufferd 2011; Blázquez Cuesta/Budría 2015), Perfektionismus (Nepon et al. 2011), Extraversion (Klein/Kotov/Bufferd 2011; Blázquez Cuesta/Budría 2015) und Gewissenhaftigkeit (Klein/Kotov/Bufferd 2011; Blázquez Cuesta/Budría 2015).

Eine psychologische Erklärung für Depression geht auf das Konzept der Selbstwirksamkeit (,self-efficacy“) von Bandura (1977) zurück. Selbstwirksamkeit bezeichnet die Erwartung einer Person ein bestimmtes Ziel aus eigener Kraft zu erreichen. Ein ähnliches Konzept ist die Kontrollüberzeugung (,locus of control"). Kontrollüberzeugung bezeichnet, inwieweit man glaubt, selbst die Kontrolle über das Auftreten oder die Folgen von Ereignissen in seinem Leben zu haben. Personen mit interner Kontrollüberzeugung glauben, dass sie selbst 
ihr „Schicksal in der Hand haben“. Personen mit externer Kontrollüberzeugung hingegen glauben, dass sie in bestimmten Situationen oder auch generell in ihrem Leben wenig selbst beeinflussen können und von äußeren Umständen (Glück, Zufall, usw.) abhängig sind. Ein erhöhtes Risiko an Depression zu leiden besteht für Personen mit geringer Selbstwirksamkeit (Bisschop et al. 2004) sowie bei externer Kontrollüberzeugung (Beekman et al. 2001; Cheng et al. 2013). Mit diesen individuellen Merkmalen sind zudem auch Angststörungen verbunden (Cheng et al. 2013; Vink/Aartsen/Schoevers 2008).

Mit dem kognitiven Modell der Depression beschreibt Beck (1987) kognitive Schemata als den Ausgangspunkt von depressiven Störungen. Zentral ist dabei die „kognitive Triade“, die (verzerrte) negative Wahrnehmung und Interpretation der Realität über sich selbst, der Umwelt und der Zukunft. „Die negativen kognitiven Schemata halten sich durch negative Erfahrungen und Lernprozesse aufrecht und führen zu einer Vielzahl von Fehlern bei der Interpretation der sozialen Realität. $\mathrm{Zu}$ diesen zählen automatische Gedanken, wie z.B. die Tendenz zu Übergeneralisierungen, das Über- oder Unterschätzen von Situationen, Alles-oder Nichts-Denken, ein Tunnelblick bzw. kognitiver Filter auf negative Aspekte und das Ignorieren positiver Aspekte“"(Scherr 2016: 78).

\subsubsection{Soziale Erklärungen}

Mirowsky und Ross (2003) haben sechs soziale Merkmale benannt, die mit Depression in Zusammenhang stehen. Diese Muster basieren auf Beobachtungen aus US-amerikanischen Studien und sind nicht zwangsweise kausal zu verstehen. Erstens spielen der sozioökonomische Status und die Bildung für gesundheitliche Ungleichheit eine bedeutende Rolle. Höhere Bildung ist mit besserer mentaler Gesundheit verknüpft (Schaan 2014; Ladin 2008; Clarke et al. 2011; Ross/Mirowsky 2006). Untersuchungen belegen auch, dass finanzielle Probleme mit einer höheren Wahrscheinlichkeit an Depressionen zu leiden verbunden sind (siehe Kapitel 3.1.1).

Zweitens kann ein Zusammenhang von Familienstand und Depression beobachtet werden. Sowohl physisch als auch mental gilt, dass Verheiratete im Vergleich zu Alleinstehenden eine bessere Gesundheit vorzuweisen haben (Carr/Springer 2010; Wright/Brown 2017). Erklärt wird dieser Zusammenhang u.a. durch die (emotionale) Unterstützung, die soziale Kontrolle, aber auch durch ökonomische Vorteile, die in einer Partnerschaft vorhanden sind (Grundy/Tomassini 2010).

Drittens lässt sich nach Ross und Mirowsky ein Zusammenhang zwischen Kindern (im Haushalt) und Depression beobachten. Obwohl das Großziehen von Kindern als soziale Norm und als freudige Angelegenheit wahrgenommen wird, zeigen Studien, dass Kinder im Haushalt das psychische Wohlbefinden nicht verbessern, teilweise sogar verschlechtern (Evenson/Simon 2005; 
Hansen/Slagsvold/Moum 2009; Hank/Wagner 2013). Mögliche Begründungen dafür könnten das erhöhte Risiko von finanziellen Problemen und mehr Konfliktpotential und Stresssituationen in der Beziehung sein (Mirowsky/Ross 2003: 92). Allerdings ist davon auszugehen, dass der Zusammenhang von Kindern und mentaler Gesundheit vom gesellschaftlichen Kontext abhängig ist und je nach den vorherrschenden Normen variiert (Huijts/Kraaykamp/Subramanian 2013).

Viertens werden in den meisten Studien Geschlechterunterschiede gefunden: Frauen sind häufiger depressiv als Männer. Als Gründe werden die traditionellen Rollen der Frau genannt, mit denen viel Stress verbunden ist: Ehefrau und Mutter. Auch heute noch übernehmen Frauen mehr Hausarbeit und eine größere Verantwortung in der Familie als Männer (Wengler/Trappe/Schmitt 2008; Schulz/Blossfeld 2006; Lenz 2009). Hinzu kommt, dass Frauen öfter ihre Berufskarriere unterbrechen oder nur in schlechter bezahlter Teilzeit beschäftigt sind, um sich der Kindererziehung zu widmen (vgl. Warren 2010; Wolf 2002; Simonson/Gordo/Titova 2011). All diese Ungleichheiten zwischen den Geschlechtern gehen zulasten der Frauen und lassen die empirisch festgestellten Unterschiede plausibel erscheinen. Dass bei der Auswertung von Umfragedaten eine höhere Quote an Depression bei Frauen festgestellt wird, könnte aber auch - zumindest teilweise - auf unterschiedliches Antwortverhalten zurückzuführen sein. Diesem Argument zufolge kennen Frauen ihre Gefühle besser und sind eher dazu bereit diese auch in einer Umfrage auszudrücken. Solche Unterschiede im Antwortverhalten wurden zwar bestätigt, allerdings erklären sie bei weitem nicht die Geschlechterunterschiede in der Prävalenz von Depression (Acciai/Hardy 2017; Mirowsky/Ross 2003: 107). Als Erklärung für die höhere Prävalenz von Depression bei Frauen sind auch Vorurteile und Rollenbilder der Geschlechter von Bedeutung. Psychische Erkrankungen gelten häufig als Frauenkrankheit und widersprechen dem Ideal von Männlichkeit (Kinzl 2013: 526). Auch das Verhalten der Ärzte ist von diesen geschlechtsspezifischen Zuschreibungen beeinflusst. Bei denselben Symptomen wird bei Frauen tendenziell häufiger eine psychische Diagnose gegeben als bei Männern, wodurch psychische Krankheiten bei Männern eher unentdeckt bleiben (Kinzl 2013: 526; Perrig-Chiello/Hutchison 2010: 210).

Fünftens scheint die Prävalenz von Depression auch in Zusammenhang mit dem Alter zu stehen. Depressionen kommen vor allem in der Jugend sowie in höherem Alter vor, im mittleren Erwachsenenalter ist die Prävalenz am geringsten (Mirowsky/Kim 2007; Clarke/Wheaton 2005). In Kapitel 2.3.6 wird genauer auf Depressionen im höheren Alter eingegangen.

Sechstens spielen Ereignisse im Lebenslauf eine wichtige Rolle. Es sind demnach nicht nur die Zustände, sondern auch die Veränderungen in den oben genannten Merkmalen - sozialer Status, Familienstand, Partnerschaft, Kinder - von Bedeutung. Früher wurde vermutet, dass jedes Ereignis mit einer Umstellung der Lebenssituation und somit mit Stress verbunden ist und daher 
(mentale) gesundheitliche Auswirkungen haben kann. Seit einiger Zeit scheint jedoch klar zu sein, dass vor allem negative empfundene Ereignisse die Wahrscheinlichkeit von Depression erhöhen. Gut belegt ist dieser Effekt für eine Trennung vom Partner und Verwitwung (Williams/Frech/Carlson 2010; Schaan 2013b), den Tod von nahen Angehörigen (Brent et al. 2009; Kristensen/Weisæth/Heir 2012) sowie für den Verlust der Arbeit (Lennon/Limonic 2010).

\subsubsection{Depression im Alter}

In der Jugend und bei jungen Erwachsenen ist die Prävalenz von Depression am höchsten. Sie nimmt im mittleren Alter ab und steigt im hohen Alter wieder an. Dieses Muster wird immer wieder berichtet, vor allem in den USA (Clarke/Wheaton 2005; Mirowsky/Kim 2007). Für Deutschland und Europa wird der Anstieg der Depressionsrate am Ende des Lebens teilweise (Wolff/Tesch-Römer 2017; Calvó-Perxas et al. 2016; Luppa et al. 2012), aber nicht in allen Studien bestätigt (Busch et al. 2013; Wittchen et al. 2010; Copeland et al. 1999), wobei Abweichungen auch durch unterschiedliche Methodik in der Erfassung der Depression zustande kommen kann. Mit den in der vorliegenden Studie verwendeten Daten lässt sich der Anstieg der (selbstberichteten) Depressionsprävalenz im höheren Alter bestätigen (siehe Kapitel 5.1). Hierbei gibt es jedoch Unterscheide zwischen den beobachteten Ländern, so ist der Anstieg in Deutschland geringer als in den meisten anderen Ländern und stimmt somit mit den bisherigen Ergebnissen überein (vgl. Wittchen et al. 2010; Busch et al. 2013).

Das Alter kann auf verschiedene Weisen betrachtet werden. Mirowsky und Ross (1992) unterscheiden fünf Ansichten des Alters, wovon jeder Aspekt zur Erklärung von Altersunterschieden in der Depression beitragen kann. Alter kann als Reife betrachtet werden. Durch Anhäufung von Lebenserfahrung und Erkenntnis sowie durch die persönliche Entwicklung geht mit höherem Alter meist auch ein ruhigeres, geordneteres und risikoärmeres Leben einher (ebd.: 188). Auftretende Probleme könnten dadurch in höherem Alter zu weniger Stress führen als in früheren Lebensphasen (vgl. Mirowsky/Ross 2001). Entgegengesetzt können der körperliche Verfall sowie die Anhäufung von Misserfolgen, Fehlern, Mängeln und Verletzungen als Verfall bezeichnet werden. So wird erklärt, warum kognitive und physische Fähigkeiten im Alter schwinden, und sich Krankheiten häufen. Biologisch wird dies mit der DisposableSoma-Theorie erklärt, wonach Altern die Kumulation von Schäden in Zellen und Organen darstellt (Westendorp/Kirkwood 2008). Die allmähliche Anhäufung von Zellschäden wird durch Stress und einen ungesunden Lebensstil beschleunigt. Die dritte Sichtweise versteht Altern als eine Abfolge von Lebensphasen. So hat nach Clarke et al. (2011) nicht das Altern per se einen Einfluss 
auf die mentale Gesundheit, sondern die Struktur des Lebenslaufes, der durch verschiedene soziale Rollen geprägt ist. Veränderungen in den Rollen sind kritische Ereignisse im Lebenslauf, die das Wohlbefinden beeinflussen können und sich auch auf die verfügbaren Ressourcen auswirken. Rollen und Positionen, die im frühen und mittleren Erwachsenenalter begonnen werden sind eher mit Ressourcen verbunden, die sich positiv auf die (mentale) Gesundheit auswirken. Im höheren Alter hingegen sind Beendigungen von Rollen bzw. Lebensphasen häufiger, die eher negative Effekte auf Ressourcen und Wohlbefinden haben, am bedeutendsten sind hierbei wohl die Erwerbstätigkeit und Ehen (vgl. Schaan 2013a: 14). Ein weiterer Aspekt von Alter ist das Überleben, wobei das Alter mit Eigenschaften zusammenhängt, die die Überlebenswahrscheinlichkeit beeinflussen. Dies führt zu einer Selektion: Eigenschaften, die ein längeres Leben ermöglichen, werden im Alter häufiger, da Personen ohne diese Eigenschaften früher sterben. Da Depression mit der Lebenserwartung in Zusammenhang steht, kann die beobachtete Korrelation von Depression und Alter verzerrt sein (vgl. Mirowsky/Ross 1992). Viele sozioökonomische Merkmale, z.B. Bildung, Einkommen und Ehe, sind sowohl mit geringerer Mortalität als auch mit einem geringeren Depressionsrisiko verknüpft. Eine Ausnahme von diesem Muster ist das Geschlecht: Frauen leiden eher an Depression, haben aber ein geringeres Mortalitätsrisiko (vgl. ebd.). Der fünfte Aspekt ist Altern als historischer Trend (oder Generation). Hierbei ist das Alter nur ein Maß für die Generation, die durch die ökonomischen und sozialen Bedingungen zu unterschiedlichen Zeiten beeinflusst ist. Jüngere Generationen profitieren von der Entwicklung der Gesellschaft und dem wachsenden Wohlstand. Vergleicht man bspw. die Bildung oder das Einkommen im selben Alter, so schneiden jüngere Generationen besser ab als Ältere. Demnach müsste höheres Alter mit mehr Depression einhergehen.

Analysiert man die Gesundheit von Menschen in höherem Alter, so ist zu bedenken, dass dies eine Lebensphase ist, die sich vom jungen und mittleren Erwachsenenalter in vielerlei Hinsicht unterscheidet. Wie in Kapitel 2.3.1 dargestellt, treten viele Erkrankungen, die typisch für das höhere Alter sind, in Zusammenhang von Depression auf. Oft ist unklar, welche dieser somatischen Krankheiten Folge oder Ursache von Depression ist. Man geht z.B. von komplexen Wechselwirkungen zwischen Depression und Demenz aus (Stoppe 2008). Auch Einschränkungen in der Mobilität und bei der Ausübung von alltäglichen Aufgaben können sowohl Depressionen begünstigen, als auch durch sie verursacht werden (Bruce 2001; Yang 2006). Die Komorbidität ist ein Grund dafür, dass Depressionen im Alter öfter chronisch sind als bei jüngeren Menschen (Stoppe 2008: 408). Weitere Gründe für die erhöhte Gefahr einer dauerhaften Depression sind die späte und inadäquate Behandlung (Stoppe 2008: 408; Gallo/Rabins 1999). Je älter der Patient oder die Patientin ist, umso seltener wird eine psychotherapeutische Behandlung durchgeführt, stattdessen kommen häufiger Medikamente zum Einsatz (Stoppe 2008: 408; Unützer et al. 
2003). Zudem werden manche Symptome einer Depression wie bspw. Schlafstörungen, Desinteresse und Rückzug, zum Teil sowohl von Patienten und Patientinnen als auch von Ärzten und Ärztinnen fälschlicherweise als ,normal für das hohe Alter" betrachtet oder es wird als Grund für die depressiven Symptome die Komorbidität ausgemacht und nur die körperlichen Erkrankungen behandelt (Gallo/Rabins 1999; Stoppe 2008: 406).

Besonders hoch ist der Anteil der depressiven Älteren in Seniorenheimen (Jongenelis et al. 2004; Seitz/Purandare/Conn 2010). In einer Studie wird anhand von Daten aus Australien bei Bewohnern von Pflegeheimen im Vergleich mit Älteren, die nicht in einem Heim wohnen, eine mehr als doppelt so hohe Depressionsrate berichtet (32 Prozent im Vergleich zu 14 Prozent) (Anstey et al. 2007). Dabei ist zu bedenken, dass der Umzug und der Aufenthalt in einer Pflegeheim sowohl Folge als auch Ursache von depressiven Symptomen sein kann (Stoppe 2008: 408).

Ein weiterer Aspekt, der sich im höheren Alter von früheren Lebensphasen unterscheidet ist die hohe Selbstmordrate. Absolut gibt es zwar bei jungen Menschen die meisten Suizide, die meisten davon finden im Alter von 20 bis 30 Jahren statt. Die Rate der Selbsttötungen ist jedoch weltweit im hohen Alter am höchsten: bei über 80-Jährigen mehr als doppelt so hoch wie im jungen und mittleren Erwachsenenalter (IHME 2017). Die Suizidrate steigt über den gesamten Lebensverlauf immer weiter an, ab einem Alter von ca. 65 Jahren ist der Anstieg der Rate besonders stark (ebd.). Depression ist einer der wichtigsten Risikofaktoren für Suizid im Alter (Conwell/Duberstein/Caine 2002; Waern/Rubenowitz/Wilhelmson 2003). Der Zusammenhang von Depression und Selbstmord ist in allen Altersgruppen sehr stark (Turvey et al. 2002), aber er wird mit zunehmendem Alter noch stärker (Conwell et al. 1996).

\subsubsection{Einfluss der finanziellen Situation auf Depression}

„Geld macht nicht glücklich, aber es beruhigt die Nerven“. Mit diesem Sprichwort kann der Einfluss der finanziellen Situation auf Depression beschrieben werden. Steht (zu) wenig Geld für das Leben zur Verfügung, so entstehen mentale Belastungen wie Stress und Zukunftsängste. Ausbleibende (finanzielle) Erfolge können psychologische Ursachen von Depression befördern, z.B. die eigene negative Wahrnehmung und geringe Selbstwirksamkeit. Verschlechterungen in der finanziellen Situation sind häufig mit Veränderungen in anderen Lebensbereichen verknüpft. Typisch hierfür sind der Verlust des Arbeitsplatzes, der Renteneintritt, eine Trennung und der Tod des Partners. Hierbei ist es wichtig, zwischen den psychischen Belastungen, die aufgrund des Ereignisses auftreten, und dem Effekt des verringerten Einkommens zu unterscheiden. Auch biologische bzw. genetische Faktoren sind in der Entstehung von De- 
pression von Bedeutung. So kann die genetische Disposition individuelle Unterschiede in der Reaktion auf Ereignisse erklären. Eine psychische Belastung stellt auch der soziale Vergleich dar. Einkommen kann als wichtiges Merkmal in der Statushierarchie angesehen werden. Verfügt man selbst über ein (dauerhaft) geringes Einkommen - und somit über einen geringen Status - so erkennt man der Rangordnungshypothese zufolge diese „Niederlage“ an, was zu depressiven Symptomen führen kann.

Ebenfalls mit dem sozialen Vergleich wird der Einfluss von Einkommensungleichheit auf die Gesundheit begründet. Bei großer Ungleichheit in der Gesellschaft ist der Stress und die Frustration - und damit die psychische Belastung - größer. Zudem ist dieser Erklärung zufolge auch die durch Ungleichheit entstehende geringe gegenseitige Unterstützung in einer Gesellschaft eine fehlende Ressource für den Umgang mit Stress und anderen Problemen. Die neomaterialistische Theorie geht hingegen davon aus, dass in Gesellschaften mit großer Ungleichheit die infrastrukturellen Bedingungen schlechter sind als in Gesellschaften mit geringerer Ungleichheit. Diese Faktoren (z.B. Umweltbedingungen, Gesundheitssystem) sind demnach auch für die psychische Gesundheit von Bedeutung. Sowohl der psychosozialen als auch der neomateriellen Hypothese nach geht eine hohe Ungleichheit mit schlechterer mentaler und physischer Gesundheit einher. Es ist jedoch zu vermuten, dass die negativen Effekte von Ungleichheit je nach Gesundheitsindikator variieren. Mentale Gesundheit ist am direktesten mit (der Wahrnehmung von) Ungleichheit verknüpft. Der ständige soziale Vergleich mit anderen, erfolgreicheren Leuten ist ein plausibler Pfad, auf dem Depressionen begünstigt werden, z.B. durch geringes Selbstwertgefühl (vgl. Wilkinson/Pickett 2017: 15f.). Auch das Argument des geringeren gesellschaftlichen Zusammenhaltes lässt sich anwenden: Durch Misstrauen und geringen sozialen Kontakt können depressive Symptome begünstigt werden. Die neomaterielle Erklärung - z.B. die Rolle des Staates bei der Gesundheitsversorgung - spielt für mentale Erkrankungen ebenso eine wichtige Rolle. Was das Angebot und die Kapazität von Behandlungsmöglichkeiten sowie die Wartezeit auf eine Behandlung von mentalen Krankheiten angeht, gibt es Unterschiede zwischen den europäischen Ländern (Barbato et al. 2016; Gutiérrez-Colosía et al. 2017). Teilweise ist es sehr schwierig für Betroffene einen Behandlungsplatz für mentale Erkrankungen zu bekommen. So variiert bspw. in den deutschen Bundesländern die Zeit von Erstkontakt zum Behandlungsbeginn von Psychotherapien zwischen 3 und 6 Monaten (BPtK 2018). 


\subsection{Einschränkungen in den Aktivitäten des täglichen Lebens}

Um physische Gesundheit zu messen wird in dieser Untersuchung ein Maß für Behinderung verwendet: die Einschränkungen in den Aktivitäten des täglichen Lebens (siehe Kapitel 4.2.2). Konzeptionell kann zwischen Aktivitäten des täglichen Lebens (ADL, ,activities of daily living“) und instrumentellen Aktivitäten des täglichen Lebens (IADL, ,instrumental activities of daily living“) unterschieden werden (Verbrugge/Jette 1994). ADL beschreibt vor allem die Körperpflege, dazu zählt u.a. sich anziehen, sich waschen und essen. Unter IADL werden vor allem Aufgaben im Haushalt zusammengefasst, z.B. kochen und einkaufen.

Die körperliche Funktionsfähigkeit ist ein besonders wichtiger Teil der Gesundheit im Alter. Sie ist mit Mortalität verknüpft (Cooper/Kuh/Hardy 2010) und vor allem ist die Eigenständigkeit im Alltag ein bedeutender Faktor für die Lebenszufriedenheit im Alter (Netuveli et al. 2006; Andersen et al. 2004). Jedes gesundheitliche Probleme ist belastend für Betroffene, jedoch werden Beeinträchtigungen in Aktivitäten oft am schlimmsten wahrgenommen (Mirowsky/Ross 2010: 376).

Einschränkungen in alltäglichen Aktivitäten können als Behinderung bezeichnet werden. In vielen Studien wird anhand dieser Kriterien die Behinderung von Personen gemessen (vgl. Topinková 2008). ${ }^{5}$ In modernen Definitionen von Behinderung sind allerdings nicht nur die medizinischen, sondern auch die sozialen Aspekte von Bedeutung. Siegrist definiert Behinderung daher als ,jede Form längerfristig bestehender körperlicher Schädigung, reduzierter geistiger Fähigkeit oder seelischer Gesundheit [...], die zu Einschränkungen des üblichen Leistungsvermögens führt und die dadurch die Teilhabe am gesellschaftlichen Leben beeinträchtigt oder erschwert" (Siegrist 2005: 305).

Verbrugge und Jette (1994) beschreiben mit dem „disablement process“ ein Schema für die Entstehung von Behinderung. Basierend auf dem Konzept von Nagi (1991) werden vier Stufen unterschieden. Physiologische Auffälligkeiten oder Abnormalitäten, die anhand medizinischer Befunde beschrieben werden können, werden als Pathologie (,,pathology“) bezeichnet. Darauf folgen Schädigungen (,impairments“), das sind Fehlfunktion und Schädigungen in Körpersystemen, z.B. im Herz-Kreislauf-System, dem Nervensystem oder dem Bewegungsapparat. Funktionale Beeinträchtigungen (,functional limitations") bezeichnen Beeinträchtigungen in grundlegenden physischen und mentalen Fähigkeiten, z.B. gehen, bücken, Treppen steigen, reden, hören, wenn

5 Eine alternative Definition richtet sich danach, ob man für die Aktivitäten Hilfe von anderen benötigt (Topinková 2008). 
sich Leute im selben Raum unterhalten, oder ein funktionierendes Kurzzeitgedächtnis. Die vierte Stufe ist Behinderung (,disability“), womit Schwierigkeiten bei Aktivitäten im täglichen Leben gemeint sind. Dazu zählen auch, aber nicht nur, ADL und IADL. Das Erwerbsleben, sowie alle anderen Bereiche im Leben, z.B. Hobbys, sind auch von Bedeutung. Einschränkungen in den ADL und IADL werden explizit als Behinderung und nicht als funktionale Beeinträchtigung angesehen. In dieser Sichtweise bezieht sich eine funktionale Beeinträchtigung nur auf die Fähigkeit eine bestimmte Aufgabe ausüben zu können (z.B. einen 10 Kilogramm schweren Gegenstand hochheben). Eine Behinderung ist hingegen ein Ausdruck oder eine Folge der funktionalen Beeinträchtigung im sozialen Kontext. Auch wenn die meisten Aktivitäten, die mit ADL und IADL bezeichnet werden, zuhause im Privaten ausgeübt werden, so sind sie dennoch Teil von sozialen Rollen und notwendig für Teilhabe am sozialen Leben (Verbrugge/Jette 1994). Zudem ist dieser Theorie zufolge nicht nur die persönliche Fähigkeit für die Definition von Behinderung von Bedeutung, sondern auch die äußeren Umstände. Behinderung ist demnach nicht Merkmal einer Person, sondern entsteht, wenn zwischen der Fähigkeit einer Person und der Anforderung einer Aktivität eine Diskrepanz besteht. Hat zum Beispiel eine Person Probleme längere Strecken zu gehen, dann hängt es auch von der Distanz zwischen Wohnung und Einkaufsmöglichkeit ab, ob sie selbst Einkäufe erledigen kann oder nicht.

Vor allem in höherem Alter treten Beeinträchtigungen in alltäglichen Aktivitäten auf: Je höher das Alter, desto größer ist die Wahrscheinlichkeit von körperlichen Einschränkungen im Alltag (Leopold/Engelhardt/Engelhartdt 2013; Andersen-Ranberg et al. 2005; Andersen-Ranberg et al. 1999). Im mittleren Erwachsenenalter ist der Anstieg gleichmäßig und eher langsam, ab ca. 65 Jahren steigt die Anzahl der betroffenen Personen deutlich stärker (Mirowsky/Ross 2010; vgl. auch Peeters et al. 2013). Dies entspricht dem Verlauf der körperlichen Funktionsfähigkeit (,functional capacity“) über den Lebenslauf wie ihn das Modell der WHO beschreibt (vgl. World Health Organization 2000). Demnach steigt die körperliche Funktionsfähigkeit zunächst an und erreicht im jungen Erwachsenenalter ihren Höhepunkt und fällt danach stetig ab. Die Rate des Abfalls der physischen Fähigkeiten ist dabei individuell verschieden und von vielen Faktoren abhängig. Unterschreitet die körperliche Funktionsfähigkeit einen bestimmten Punkt, dann kann von Behinderung gesprochen werden. Der Schwellenwert der Behinderung ist dabei - ähnlich wie bei dem Modell von Verbrugge und Jette - von der Umwelt abhängig.

In der Entwicklung von Behinderungen im Alter kann zwischen progressiver und plötzlicher (,,catastrophic“) Behinderung unterschieden werden (Ferrucci et al. 1996). In einer Studie mit Daten aus den USA aus den 1980er Jahren wurde ungefähr die Hälfte der Behinderungen als allmählich fortschreitende Entwicklung und die andere Hälfte im Zusammenhang mit plötzlichen Ereig- 
nissen kategorisiert (Ferrucci et al. 1996). In anderen Studien wird jedoch häufiger von einer progressiven Entstehung von Behinderungen berichtet, wobei die progressive Entwicklung besonders häufig in sehr hohem Alter ab 85 Jahren zu beobachten ist (Guralnik et al. 2001). ${ }^{6}$ Obwohl es große individuelle Unterschiede in der Entwicklung von Behinderungen gibt, wird dennoch ein gewisses Muster bei progressiven Pfaden beschrieben (vgl. Manini 2001). Legt man das Modell von Verbrugge und Jette zugrunde, so wird eine ,pre-clinical““ Phase der Behinderung beschrieben, die zwischen der Entstehung der funktionalen Beeinträchtigungen und der eigentlichen Behinderung verortet wird. Diese Phase zeichnet sich dadurch aus, dass Aktivitäten ausgeführt werden können, sie jedoch weniger häufig ausgeübt werden oder eine Anpassung der Methode (z.B. durch Hilfsmittel) stattfindet (ebd.). Die plötzliche Entstehung von körperlichen Beeinträchtigungen ist häufig mit Krankheiten, z.B. Krebserkrankungen, oder einem kurz darauf folgenden Tod verbunden (Lunney et al. 2003; Gill et al. 2010). Dennoch ist es durchaus üblich, dass auch im Alter Verbesserungen in der körperlichen Leistungsfähigkeit bzw. Erholung von Behinderungen möglich sind (Hardy/Gill 2004; Litwin/Stoeckel 2013). Allerdings ist die Erholung in vielen Fällen nicht von Dauer. Vor allem bei längeren Phasen von Behinderung ist eine erneut auftretende Einschränkung wahrscheinlich (Hardy/Gill 2004).

Nach dem „disablement process“ ist die Ursache einer Einschränkung in Aktivitäten des täglichen Lebens sowohl in den individuellen funktionalen Beeinträchtigungen, wie auch in den Umweltbedingungen zu suchen. Solche Umweltfaktoren betreffen oftmals die Ausstattung der Wohnung oder Hilfsmittel, Beispiele hierfür sind eine barrierefreie Wohnung, eine Brille und ein Hörgerät. Diese Art der Umweltfaktoren ist mit finanziellen Ressourcen verknüpft, die aus dem Privatvermögen oder von staatlichen Einrichtungen stammen können. Durch das Sozialsystem, aber auch durch die Förderung von Barrierefreiheit, hat der Staat somit direkten Einfluss auf das Ausmaß der Behinderung der Bürger (vgl. World Health Organization 2007). Die andere Ursache von Behinderung ist die funktionale Beeinträchtigung der Individuen. Die staatlichen Möglichkeiten sind hier deutlich geringer. Zwar kann durch die Gesundheitsversorgung und Präventionsmaßnahmen auf die Gesundheit der Bürger Einfluss genommen werden, jedoch ist zu vermuten, dass der größere Teil des staatlichen Einflusses über die Regelung des Zugangs zu Rehabilitationsmaßnahmen erfolgt.

Die bedeutendsten Auslöser von funktionalen Beeinträchtigungen sind ohne Zweifel Krankheiten und Unfälle. Besonders am Lebensende ist die körperliche Funktionsfähigkeit sehr stark durch Krankheiten wie Krebs und Or-

6 Hierbei sind jedoch Selektionsprozesse zu beachten. Betroffene von plötzlicher Erkrankung haben vermutlich eine höhere Mortalitätsrate und erreichen das hohe Alter daher unwahrscheinlicher. 
ganversagen beeinflusst (Lunney et al. 2003). Eine weitere Krankheit, die Einschränkungen in Aktivitäten begünstigen kann, ist Depression (Hays et al. 1997; Stuck et al. 1999; Penninx 1998; Connolly/Garvey/McKee 2017). Adipositas erhöht das Risiko von körperlichen Einschränkungen, vor allem des Unterkörpers (Backholer et al. 2012; Stuck et al. 1999; Ferraro/Kelley-Moore 2003; Ferraro et al. 2002; Peeters et al. 2004). Aber auch Untergewicht ist ein Faktor der die Entstehung von Behinderungen begünstigt (Ferraro et al. 2002; Stuck et al. 1999).

Auch das Gesundheitsverhalten - Rauchen, Alkoholkonsum und vor allem physische Aktivität - spielt eine Rolle bei der Entstehung von körperlichen Einschränkungen (Stuck et al. 1999; Tak et al. 2013). Ohne aktive Gegenmaßnahmen verliert der menschliche Körper in der zweiten Lebenshälfte zunehmend an Muskelmasse. Unabhängig vom Geschlecht verringern sich ab einem Alter von ca. 50 Jahren die Skelettmuskeln, wobei vor allem der Unterkörper betroffen ist (Janssen et al. 2000; Gallagher et al. 1997; vgl. auch Frontera et al. 2000). Studien zeigen auch, dass die Kraft in den Händen mit dem Alter geringer wird (Frederiksen et al. 2006: 561; Andersen-Ranberg et al. 2009; Rantanen et al. 1998) und die Greifkraft ein Prädiktor für spätere körperliche Einschränkungen ist (Rantanen 1999). Diese Ergebnisse machen deutlich, warum physische Aktivität für die Entstehung von körperlichen Beeinträchtigungen von großer Bedeutung ist (vgl. Stuck et al. 1999). Werden Muskeln nicht beansprucht, so verlieren sie an Masse und Stärke. Der Abbau von Muskelmasse bei Inaktivität schreitet dabei viel schneller voran, als der Muskelaufbau durch körperliches Training. Ein Experiment hat gezeigt, dass sowohl bei jungen wie auch bei älteren Männern die Schwächung der Muskeln, die durch eine zweiwöchige Ruhigstellung der Beine entsteht, durch ein sechswöchiges Training nicht komplett ausgeglichen werden kann (Vigelsø et al. 2015). Was die Einschränkungen in Aktivitäten im Alter betrifft, so gibt es Hinweise darauf, dass vor allem die Muskelstärke und nicht die Muskelmasse von Bedeutung ist (Newman et al. 2006; Visser et al. 2005).

Einen unmittelbaren Einfluss von finanzieller Situation auf funktionale Beeinträchtigungen ist nicht zu erwarten. Allerdings sind dennoch Zusammenhänge und Pfade zwischen finanzieller Situation und Behinderung denkbar. Wie oben beschrieben führen finanzielle Probleme zu Sorgen und Stress wodurch der Körper reagiert, z.B. mit Ausschüttung von Hormonen. Auch wenn kein sofortiger Effekt sichtbar ist, kann dies langfristig auch auf die körperliche Leistungsfähigkeit wirken. Zudem gibt die finanzielle Situation vor, welche Hilfsmittel angeschafft werden können, um eine Beeinträchtigung auszugleichen. Und auch mögliche Behandlungen können kostspielig und daher nicht für alle Statusgruppen in gleichem Maße zugänglich sein.

Auch ein möglicher Einfluss von Einkommens- und Vermögensungleichheit auf funktionale Beeinträchtigungen ist durch langfristig wirkende psychische Belastungen denkbar. Chronischer Stress - wie er der psychosozialen 
Theorie nach durch den sozialen Vergleich und den Wettbewerb entsteht - ist für den Ausbruch vieler Erkrankungen (mit-) verantwortlich. Dass in ungleichen Gesellschaften vermehrt körperliche Beeinträchtigungen auftauchen ist somit plausibel. Aber nicht nur bei der Entstehung, sondern auch bei der Regeneration von einer Krankheit und für das Leben mit einer Krankheit sind die in den Theorien genannten Mechanismen von Bedeutung. Zum einen ist Unterstützung im näheren Umfeld eine wichtige Ressource für die Regeneration (vgl. Berkman/Krishna 2014). Zum anderen ist der Zugang zu Gesundheitsversorgung und Rehabilitationsmaßnahmen sowie staatliche Unterstützung im Pflegefall ein wesentlicher Faktor für eine bestmögliche Erholung.

\subsection{Subjektive Gesundheit}

Neben Depression und Einschränkungen in Aktivitäten wird als dritter Indikator die subjektive Gesundheit untersucht. Zum einen, weil die subjektive Gesundheit in vielen Studien verwendet wird und somit die Vergleichbarkeit der hier dargestellten Analysen erhöht wird. Zum anderen ist die subjektiv abgefragte Gesundheit ein Maß, das physische und mentale Gesundheit vereint, da nach dem allgemeinen Gesundheitszustand gefragt wird.

Jylhä (2009) hat ein theoretisches Modell vorgeschlagen, das versucht die Bewertung der eigenen Gesundheit zu erklären und diesen Vorgang als kognitiven Prozess beschreibt. Das Modell unterscheidet drei Ebenen, auf denen Entscheidungen getroffen werden müssen. Alle diese Entscheidungen sind dabei nicht nur von den individuellen Informationen und Empfindungen abhängig, sondern auch von kontextuellen Faktoren. Mit der Frage nach dem allgemeinen Gesundheitszustand konfrontiert, muss der oder die Befragte zunächst entscheiden was „Gesundheit“ überhaupt ist, also welche Aspekte der Gesundheit für die Bewertung eine Rolle spielen. Mögliche Aspekte sind z.B. medizinische Diagnosen, körperliche Leistungsfähigkeit, empfundene Symptome, körperliche Empfindungen, verschriebene Medikamente, Krankschreibungen sowie bekannte Risikofaktoren für die zukünftige Gesundheit (Verhalten, Gene). Für die individuelle Definition von Gesundheit ist auch der gesellschaftliche und kulturelle Rahmen von Bedeutung, da dieser eine Orientierung bietet (vgl. Jürges 2007). Ist die eigene Gesundheit definiert, so muss im zweiten Schritt erörtert werden, wie sie bewertet werden kann in Anbetracht des eigenen Alters, der Personen im Umfeld sowie dem eigenen früheren und zukünftig erwartbaren Gesundheitszustand. Im letzten Schritt werden die verfügbaren Antwortkategorien mit der Bewertung der eigenen Gesundheit verglichen und eingeordnet, wobei auch Normen von Bedeutung sind (soziale Erwünschtheit, Angemessenheit von positiven und negativen Meinungen, gängige Interpretation der Skala) (Jylhä 2009). Die Bezeichnung dieser Schritte 
als kognitiven Prozess meint nicht, dass die Antwort zwingend aufgrund von bewusster, logischer Schlussfolgerung entsteht, sondern, dass zur Einschätzung der eigenen Gesundheit eine Auswahl und Verarbeitung von Informationen und deren Interpretation notwendig ist (ebd.: 308). Dieses Modell zeigt, wie viele Entscheidungen für die Beantwortung einer ,einfachen“ Frage nach dem Gesundheitszustand nötig sind und erklärt somit, weshalb subjektive Bewertungen der gleichen objektiven Situation individuell stark abweichen können. Zudem bezieht es Kontextfaktoren ein und erklärt somit unterschiedliches Antwortverhalten zwischen Ländern, das durch Normen oder auch durch Sprache (Formulierung der Frage und der Antwortmöglichkeiten) zustande kommen kann (vgl. Jürges 2007).

Es gibt Unterschiede bei der genauen Formulierung der Frage und bei den möglichen Antwortkategorien. Die in den USA am häufigsten verwendeten Antwortmöglichkeiten sind ,ausgezeichnet“, ,sehr gut“, ,gut“, ,mittelmäßig“ und ,schlecht“. Von der WHO (Bruin/Picavet/Nossikov 1996) sowie einer Expertengruppe (Robine/Jagger/The Euro-Reves Group 2003) wird hingegen die Variante mit den Antwortmöglichkeiten „sehr gut", „gut“, „mittelmäßig“, „schlecht", ,sehr schlecht" empfohlen. Es existieren auch andere, weniger geläufige Variationen (vgl. Jylhä 2009: 308). Auch wenn die verschiedenen Skalen nicht genau miteinander Vergleichbar sind, so zeigen Auswertungen, dass sie prinzipiell das gleiche latente Konstrukt messen (Jürges/Avendano/Mackenbach 2008).

Die subjektive Bewertung der Gesundheit führt zu Problemen bei der Interpretation und Vergleichbarkeit der Antworten. Wenn Personen ihrem Bewertungsstil treu bleiben ist es jedoch für Längsschnittuntersuchungen kein Problem, da hierbei nur individuelle Veränderungen betrachtet werden (siehe Kapitel 4.6). Es gibt allerdings Hinweise darauf, dass Befragte teilweise inkonsistente Antworten geben und die Erfassung der subjektiven Gesundheit zu Messfehlern führt (vgl. Gunasekara/Carter/Blakely 2012). In einer australischen Studie wurde die subjektive Gesundheit in einem Fragebogen zweimal erfragt, wobei bei $28 \%$ der Befragten die Antworten nicht übereinstimmten (Crossley/Kennedy 2002; vgl. auch Jürges/Avendano/Mackenbach 2008).

Trotz dieser Nachteile wird die subjektive Gesundheit in vielen Studien verwendet und als valides Maß angesehen (Benyamini 2011). Als Begründung wird angegeben, dass die subjektiv abgefragte Gesundheit die Multidimensionalität von Gesundheit erfasst und sowohl mit Mortalität (Idler/Benyamini 1997; DeSalvo et al. 2006) als auch anderen objektiven Gesundheitsindikatoren korreliert (Jylhä et al. 2001; Christian et al. 2011; Pinquart 2001).

Da das aktuelle Wohlbefinden und psychische Belastungen die Beurteilung der eigenen Gesundheit beeinflussen, ist davon auszugehen, dass die finanzielle Situation einen direkten Effekt auf die subjektive Gesundheit hat. Gleiches gilt auch für die Einkommens- und Vermögensungleichheit. Psychische Belastungen durch eine starke Wettbewerbssituation, Vergleiche mit anderen und 
mangelnde soziale Unterstützung können direkt auf das Wohlbefinden und die subjektiv empfundene Gesundheit wirken. Hinzu kommen langfristigen Folgen einer dauerhaften Belastung durch Stress und Frustration, die sich auch in physischen Symptomen niederschlagen können. 


\section{Forschungsstand}

In diesem Kapitel werden für alle aufgeworfenen Forschungsfragen die bisherigen relevanten Forschungsergebnisse dargestellt. Dass Menschen mit geringen finanziellen Mitteln im Durchschnitt eine schlechtere Gesundheit und eine höhere Sterblichkeit vorweisen, ist durch eine Vielzahl von wissenschaftlichen Studien belegt. Allerdings existieren bisher nur wenige Studien, die den $\mathrm{Zu}-$ sammenhang von finanzieller Situation und Gesundheit im Längsschnitt betrachten. Und davon wiederum nur wenige, die Armutsindikatoren verwenden, die nicht auf Einkommen basieren oder speziell ältere Menschen untersuchen (siehe Kapitel 3.1). Auch zu der Hypothese der Verlustaversion - die Unterscheidung der Effekte von Verbesserung und Verschlechterung der finanziellen Lage - existieren bislang wenige Erkenntnisse. Die Studien, die hierfür von Bedeutung sind werden in Kapitel 3.2 zusammengefasst. Der Abschnitt 3.3 beschreibt den Forschungsstand zum Zusammenhang von Einkommensungleichheit und Gesundheit. Die Vermögensungleichheit wird nur sehr kurz thematisiert, da bislang fast keine Forschung auf diesem Feld existiert.

\subsection{Finanzielle Probleme und Gesundheit}

Es existieren sehr viele Studien, die im Querschnitt zeigen, dass finanzielle Schwierigkeiten oder ein niedriges Einkommen mit der Gesundheit korrelieren (z.B. Terraneo 2017; Foulds/Wells/Mulder 2014; Lampert/Kroll 2006; Präg/Mills/Wittek 2014; Hong/Knapp/McGuire 2011; Meltzer et al. 2010). Ob die gesundheitlichen Unterschiede mit zunehmendem Alter eher größer werden, stabil bleiben oder geringer werden ist umstritten (vgl. Leopold/Engelhardt/Engelhartdt 2013; Huisman/Kunst/Mackenbach 2003). Fest steht jedoch, dass dieser Zusammenhang auch im hohen Alter besteht (Dahl/Birkelund 1997; Huisman/Kunst/Mackenbach 2003). In diesem Abschnitt wird der Forschungsstand bezüglich der drei Gesundheitsindikatoren - Depression, Einschränkungen in Aktivitäten, subjektive Gesundheit - genauer dargestellt. Den Forschungsfragen entsprechend liegt das Hauptaugenmerk hierbei auf Längsschnittanalysen, die die gesundheitlichen Folgen von finanziellen Problemen untersuchen.

\subsubsection{Depression}

In der sozialwissenschaftlichen Forschung existieren zahlreiche Studien, die verschiedene psychische Krankheiten analysieren, wie z.B. Angststörungen 
(Vink/Aartsen/Schoevers 2008) und Schizophrenie (Read 2010). Am häufigsten wird jedoch Depression untersucht. Zum einen, da es eine relativ häufige Krankheit ist, und so auch in Zufallsstichproben eine ausreichende Fallzahl zur Verfügung steht. Andererseits kann die Abfrage von depressiven Symptomen relativ unkompliziert in sozialwissenschaftlichen Umfragen integriert werden (vgl. Radloff 2016; Prince et al. 1999).

Wie auch für viele andere Gesundheitsindikatoren ist der Zusammenhang von finanziellen Problemen und Depression im Querschnitt vielfach belegt worden (z.B. Kuo/Chiang 2013; Kim/Park/Kim 2018; Franzese 2015; Madianos et al. 2011). Im Folgenden werden die wichtigsten Studien vorgestellt, die den Zusammenhang von finanziellen Notlagen und Depression im Zeitverlauf betrachten. Im Vergleich zu Querschnittsuntersuchungen ist es ein Vorteil, von denselben Personen Informationen zu mehreren Zeitpunkten zur Verfügung zu haben. Um den vermuteten kausalen Zusammenhang besser zu beschreiben, kann mit diesen Daten die zeitliche Abfolge berücksichtigt werden (vgl. z.B. Carter et al. 2009). So beschreibt eine Studie anhand von Daten aus den USA, dass die Zwangsvollstreckung des Hauses zwischen zwei Befragungszeitpunkten die Anzahl der depressiven Symptome bei der zweiten Befragung erhöht (McLaughlin et al. 2012). Mit ähnlicher Vorgehensweise zeigt eine australische Studie zunächst, dass finanzielle Schwierigkeiten bei der ersten Befragung einen Einfluss auf das Depressionsrisiko bei der Wiederholungsbefragung haben. Dieser Effekt ist jedoch nicht mehr vorhanden, sobald in den Berechnungen die Depression zum ersten Zeitpunkt kontrolliert wird (Butterworth/Rodgers/Windsor 2009). Bei der Auswertung des Southampton Women's Survey stellen Dunn et al. (2008) fest, dass Armut, gemessen anhand des Bezugs von Sozialleistungen, die Wahrscheinlichkeit erhöht, innerhalb von zwei Jahren eine Depression zu entwickeln. Die subjektiv erfasste finanzielle Belastung ist ebenfalls mit einer erhöhten Depressionsrate verknüpft, wobei dieser Zusammenhang unter Berücksichtigung anderer Faktoren nicht mehr bestätigt wird.

Noch besser als die Veränderung zwischen zwei Zeitpunkten mit Querschnittsmethoden zu analysieren, ist die Verwendung von Methoden, die für die Analyse von Längsschnittinformationen entwickelt wurden, um die vorhandenen Informationen bestmöglich zu nutzen. Mit dem Fixed-Effects-Modell werden nicht Unterschiede zwischen den Befragten, sondern nur individuelle Veränderungen im Zeitverlauf analysiert (siehe Kapitel 4.6.3). FixedEffects-Berechnungen lassen zwar auch nicht ohne weiteres kausale Interpretationen von beobachteten Zusammenhängen zu, allerdings kommt man damit dem kausalen Effekt näher als mit Querschnittsberechnungen. Unveränderliche Eigenschaften werden kontrolliert, bzw. spielen keine Rolle, somit ist dies eine gute Methode, um Veränderungen in der Gesundheit zu analysieren (v.a. im Vergleich zu Querschnittsmodellen). 
Für die Frage, wie sich Veränderungen in der finanziellen Situation auf Depressionen oder depressive Symptome auswirken, gibt es einige Studien, die Längsschnittdaten mit Fixed-Effects-Modellen auswerten. Die Ergebnisse dieser Studien sprechen einheitlich dafür, dass finanzielle Probleme mit einem erhöhten Depressionsrisiko verbunden sind. Heflin und Iceland (2009) untersuchen den Zusammenhang von materiellen Notlagen und Depression bei Müttern. Die Analysen basieren auf Daten einer US-amerikanischen Umfrage, bei der die Mütter zu zwei Zeitpunkten - ein Jahr und drei Jahre nach der Geburt - befragt wurden. Zwei der fünf Indikatoren materieller Not - Probleme, Rechnungen bezahlen zu können und Abschaltung des Telefons - gehen in der Längsschnittanalyse mit erhöhter Wahrscheinlichkeit von Depression einher. Für eine andere Studie wurden in einer ländlichen Gegend Kanadas dieselben Personen zu drei Zeitpunkten, jeweils mit neun Monaten Abstand, zu ihrer Gesundheit und finanziellen Lage befragt. Die Analyse dieser Daten zeigt auch hier, dass finanzielle Schwierigkeiten mit mehr depressiven Symptomen korrelieren (Frank/Davis/Elgar 2014). Auch eine Panelstudie aus Großbritannien kommt zu dem Ergebnis, dass finanzielle Probleme die Wahrscheinlichkeit erhöhen eine Depression zu bekommen (Bridges/Disney 2010). Monserud und Markides (2017) kommen bei der Berechnung von Wachstumskurven anhand von sieben Befragungswellen einer Längsschnittbefragung von Mexikanern, die in den USA leben, zu dem Ergebnis, dass finanzielle Belastungen mit einer höheren Anzahl depressiver Symptome einhergehen. Auch in den Arbeiten von Lorant et al. (2007) und Mckenzie et al. (2014) wird ein Zusammenhang von verschiedenen Armutsindikatoren und Depression anhand von FixedEffects-Berechnungen bestätigt (beide Studien werden in Kapitel 3.2 genauer beschrieben). Taylor et al. (2007) untersuchen anhand von 13 Befragungswellen des British Household Panel Survey die Auswirkungen einer bestimmten finanziellen Notsituation: Probleme bei der Zahlung der Wohnung (Miete oder Hypothek). Zahlungsprobleme und Verzug der Zahlungen sind mit mehr Symptomen einer Depression verbunden, wobei für Frauen die Effektstärken von andauernden Problemen mit Zahlungen für die Wohnung deutlich stärker sind als andere Indikatoren der finanziellen Situation. Die Auswertung von australischen Längsschnittdaten hat ergeben, dass finanzielle Risikofaktoren wie Arbeitsplatzunsicherheit, Unzufriedenheit mit der finanziellen Situation und fehlende Rücklagen für Notfälle die mentale Gesundheit (gemessen anhand eines Index, der vor allem aus depressiven Symptomen besteht) verschlechtert (Rohde et al. 2016).

Es existieren bereits Studien, die mit Längsschnittdaten und -methoden die Veränderungen der depressiven Symptome im höheren Alter analysieren. In einer Studie mit Daten der Longitudinal Aging Study Amsterdam wird untersucht, wie sich verschiedene Ereignisse im höheren Alter auf die mentale Gesundheit auswirken (Lindeboom/Portrait/van den Berg, Gerard J 2002). Drei Messungen von Personen ab 55 Jahren im Zeitraum von 1992 bis 1999 werden 
anhand von Fixed-Effects Modellen analysiert. Das Auftreten von finanziellen Problemen geht mit einem erhöhten Wert auf der CES-D Depressionsskala einher. Eine Stärke dieser Studie ist, dass auf eine Vielzahl von Ereignissen kontrolliert wird, die die mentale Gesundheit und zum Teil auch die finanzielle Situation beeinflussen, wie z.B. Trennung vom Partner, Verwitwung, Tod von nahen Verwandten, Verlust des Arbeitsplatzes und verschiedene Krankheiten. Eine Längsschnittuntersuchung aus Südkorea beschreibt, welche Auswirkungen verschiedene Notlagen - ausgelassene Mahlzeiten, Schwierigkeiten beim Bezahlen von Rechnungen, Miete, Heizung und medizinischer Versorgung für die mentale Gesundheit von älteren Menschen haben $(\mathrm{Kim} / \mathrm{Shim} / \mathrm{Lee}$ 2016). Die Auswertungen belegen einen Zusammenhang von materieller Not und Depression, wobei dieser Effekt für Personen, die älter als 65 Jahre alt sind und alleine wohnen, am stärksten ist. Mirowsky und Ross (2001) untersuchen mit Daten einer Telefonumfrage aus den USA, wie sich der Einfluss von finanziellen Problemen auf Depression im Alter entwickelt. Die Ergebnisse zeigen auf, dass finanzielle Probleme mit Depression verknüpft sind, dieser $\mathrm{Zu}-$ sammenhang jedoch mit zunehmendem Alter geringer wird. Hiilamo \& Grundy (2018) finden in ihrer Analyse von Personen ab 50 Jahren einen positiven Zusammenhang von Verschuldung und Depression.

Mit der Forschungsfrage nach den Auswirkungen von finanzieller Not eng verknüpft sind Analysen zu Änderungen im Einkommen. Zwar legen Untersuchungen sowohl mit Quer- (Foulds/Wells/Mulder 2014; Franzese 2015; French/McKillop 2017) als auch mit Längsschnittdaten (Rohde et al. 2016; McKenzie et al. 2014; Bridges/Disney 2010; Adena/Myck 2014) nahe, dass die Korrelation mit Gesundheit stärker ist, wenn Maße für die finanzielle Situation verwendet werden, die nicht auf Einkommen basieren, dennoch sollen einige der Studien zum Zusammenhang von Einkommen und einkommensbasierten Maßen mit Depression dargestellt werden. Frijters und Ulker (2008) können bspw. anhand der US-amerikanischen Health and Retirement Study feststellen, dass eine Erhöhung des Einkommens mit einer Verringerung der depressiven Symptome einhergeht. Auch in einer Längsschnittuntersuchung von Müttern, die von der Geburt des Kindes an für drei Jahre befragt wurden, sind sowohl das Einkommen, als auch ein einkommensbasiertes Maß von Armut mit depressiven Symptomen korreliert (Dearing/Taylor/McCartney 2004). Jones und Wildman (2008) analysieren mit elf Wellen des British Household Panel Survey wie das Haushaltseinkommen und relative Deprivation (ein Maß basierend auf dem Haushaltseinkommen und der Einkommensverteilung auf nationaler Ebene) auf das psychische Wohl auswirkt. Einen Zusammenhang von Einkommen mit der psychischen Verfassung kann in den Längsschnittanalysen nur für Frauen festgestellt werden. Eine Längsschnittstudie mit dem Sozioökonomischen Panel legt dar, dass relative Deprivation, gemessen anhand des eigenen Einkommens im Vergleich zum Einkommen einer Vergleichsgruppe, einen negativen Einfluss auf die mentale Gesundheit hat (Blázquez 
Cuesta/Budría 2015). Da in der Analyse auch das Haushaltseinkommen berücksichtigt wird, können die Ergebnisse als Hinweis aufgefasst werden, dass die negativen Folgen von geringem Einkommen teilweise auf nachteilige soziale Vergleiche zurückzuführen sind (ebd.: 23).

Im Vergleich zu der Vielzahl an Untersuchungen zum Einkommen gibt es weniger Forschung, die sich den Zusammenhang von Gesundheit mit dem Vermögen anschaut. Adena und Myck (2014) berichten bei der Auswertung des Survey of Health, Ageing and Retirement in Europe, dass ein Armutsindikator, der auf dem Vermögen basiert, stärker mit Veränderungen in der Gesundheit korreliert als die Einkommensarmut. Zimmerman und Katon (2005) analysieren drei Befragungswellen des in den 1990er Jahren in den USA erhobenen National Longitudinal Survey of Youth. Anhand von Panelanalysen kann kein Zusammenhang von Depression und Einkommen festgestellt werden, allerdings beschreiben sie einen Zusammenhang mit einem Indikator für Vermögen. Eine alternative Analysemethode, der Instrumentalvariablen-Ansatz, kann einen Einfluss von dem Vermögensmaß jedoch nicht bestätigen (ebd.).

Ein anderer Zweig in der Literatur untersucht die gesundheitlichen Folgen von Einkommensschocks. In Studien werden hierfür oft Lotteriegewinne analysiert. Die Gewinne sind unvorhersehbar und somit nicht wie die sonstigen Einkommensveränderungen mit anderen Ereignissen - z.B. Kündigung, neuer Arbeitsplatz, Beförderung, Renteneintritt - verknüpft. Die Ergebnisse dieser Studien sind uneinheitlich. So wird zum Teil von besserer mentalen Gesundheit nach einem Gewinn berichtet (Gardner/Oswald 2007; Apouey/Clark 2015), teilweise jedoch auch von einer Verschlechterung der mentalen Gesundheit (Raschke 2015; Nisslé/Bschor 2002) oder keinem Zusammenhang (Lindqvist/Östling/Cesarini 2018).

Schwandt (2014) nutzt die Schwankungen des Aktienmarkts aus, um den Effekt von Veränderungen im Vermögen auf die Anzahl depressiver Symptome von älteren Menschen zu untersuchen. Ähnlich wie bei Lotteriegewinnen lässt der Zusammenhang von Aktienkurs und psychischer Verfassung nur eine kausale Interpretation zu: Die psychische Gesundheit kann durch das Auf und $\mathrm{Ab}$ der Aktienkurse beeinflusst werden, die Entwicklungen an den Börsen ist hingegen nicht von der Gesundheit des Aktienbesitzers abhängig. Die Analysen zeigen, dass höhere Aktienkurse und die damit einhergehende Vermehrung des Vermögens mit geringeren Werten auf dem verwendeten Depressionsindex einhergeht (ebd.).

\subsubsection{Physische Gesundheit}

Es existiert eine Vielzahl an Studien, die Einkommen oder finanzielle Probleme in Zusammenhang mit physischer Gesundheit untersuchen, wobei in der Regel eine Korrelation aufgezeigt werden kann. Beispielsweise kommt Deindl 
(2015) zu dem Ergebnis, dass finanzielle Probleme und Stress im Lebenslauf einen Einfluss darauf haben im späteren Leben an einer Herzerkrankung zu leiden. Es ist zudem bekannt, dass ein höheres Einkommen mit geringerem Mortalitätsrisiko einhergeht (Lampert/Kroll 2006; Martikainen/Valkonen/ Moustgaard 2009; Klein/Unger 2001; Backlund/Sorlie/Johnson 1996; Tjepkema/Wilkins/Long 2013), wobei jedoch ,vor allem ein Leben in Armut gesundheitsabträglich ist, während sich Einkommensunterschiede innerhalb des oberen Einkommensbereichs nur eher marginal auf die Mortalität auswirken“ (Klein/Unger 2001: 146; vgl. auch Backlund/Sorlie/Johnson 1996; Tjepkema/Wilkins/Long 2013; Adena/Myck 2014). Seltener untersucht wurde das Mortalitätsrisiko in Abhängigkeit von finanziellen Problemen. Szanton et al. (2008) untersuchen diesen Zusammenhang bei älteren Frauen und beobachten dabei auch unter der Kontrolle des Einkommens ein erhöhtes Mortalitätsrisiko bei finanziellen Problemen.

\subsubsection{Einschränkungen in Aktivitäten des täglichen Lebens}

Da körperliche Einschränkungen in Aktivitäten vor allem im höheren Alter auftreten, besteht der Großteil der Forschung aus Analysen von Daten, die sich auf die ältere Bevölkerung spezialisieren. Die meisten Analysen, die dieses Thema in Zusammenhang mit finanziellen Ressourcen untersuchen, wurden zudem anhand von Querschnittsberechnungen durchgeführt.

Eine Studie, die Einschränkungen in instrumentellen Aktivitäten und der Mobilität bei älteren Menschen in den USA und Europa untersucht, zeigt auf, dass mit hohem Vermögen geringere Einschränkungen verknüpft sind (Wahrendorf/Reinhardt/Siegrist 2013). Ein Zusammenhang von Vermögen, Einkommen und Einschränkungen in Aktivitäten wird auch für Südkorea (Park/Jung/Lee 2009), Griechenland (Tsimbos/Verropoulou 2008) und Deutschland (Knesebeck et al. 2003) berichtet. Studien aus Polen (ĆwirlejSozańska et al. 2018) und den Niederlanden (Gobbens/van Assen 2014) kommen zu dem Ergebnis, dass Probleme bei der Ausführung von alltäglichen Aktivitäten mit dem Einkommen bei bivariaten Analysen korrelieren, dieser $\mathrm{Zu}-$ sammenhang jedoch nicht Bestand hat, wenn für andere Einflussfaktoren kontrolliert wird.

Es existieren deutlich weniger Querschnittstudien, die körperliche Einschränkungen mit Armutsindikatoren verknüpfen, die nicht auf Einkommen oder Vermögen basieren. So stellen Mottram et al. (2008) in einer regionalen Studie aus England fest, dass Probleme mit dem Einkommen über die Runden zu kommen mit einer erhöhten Wahrscheinlichkeit von Einschränkungen in der Mobilität korrelieren. Auch Untersuchungen aus den USA zeigen, dass finanzielle Probleme mit Einschränkungen in Aktivitäten des täglichen Lebens 
im Zusammenhang stehen (Szanton/Thorpe/Whitfield 2010; Angel et al. 2003).

Ebenfalls ein kaum erforschtes Gebiet sind Längsschnittstudien von Einschränkungen in Aktivitäten. Die vorhandenen Arbeiten werden kurz dargestellt, wobei keine davon Fixed-Effects-Analysen anwendet. Adena und Myck (2014) untersuchen mit Daten des Survey of Health, Ageing and Retirement in Europe die Veränderung der Gesundheit zwischen zwei Zeitpunkten. Unter anderem betrachten sie die Anzahl der Einschränkungen bei Aktivitäten des täglichen Lebens, wobei ein binärer Indikator für schlechte Gesundheit (drei oder mehr Einschränkungen) für die Analysen verwendet wird. Neben Indikatoren für Einkommensarmut und Vermögensarmut ist die „subjektive Armut“ Probleme mit dem verfügbaren Einkommen über die Runden zu kommen - die zentrale unabhängige Variable. Die Ergebnisse von logistischen Regressionen zeigen, dass subjektive Armut und Vermögensarmut zum ersten Zeitpunkt die Wahrscheinlichkeit einer Verschlechterung der Gesundheit erhöhen und eine Verbesserung der Gesundheit unwahrscheinlicher machen. Ein ähnliches Vorgehen verwenden McMunn/Nazroo/Breeze (2009) mit zwei Befragungswellen der English Longitudinal Study of Ageing. Sie können belegen, dass bei Personen ab 50 Jahren das Vermögen, das Wohneigentum, wie auch das Einkommen mit der Entstehung von Einschränkungen in Aktivitäten des täglichen Lebens negativ korrelieren, wobei jedoch der Gradient für das Einkommen am geringsten ist. Matthews et al. (2005) untersuchen Daten einer Studie aus Großbritannien, in der Personen ab 75 Jahre zwischen 1988 und 2003 bis zu sieben Mal zu ihrer Gesundheit befragt wurden. Zur Erfassung der sozioökonomischen Situation wurde erhoben, ob die Befragten ihr Einkommen als angemessen erachten oder ob sie Probleme haben, damit auszukommen. Als Indikator für Behinderung dient die Information, ob die Befragten mindestens eine Einschränkung in fünf erfragten Aktivitäten des täglichen Lebens haben. In einer Querschnittsberechnung der Daten der ersten Befragung aus dem Jahr 1988 kann ein Zusammenhang von einem zu niedrig empfundenen Einkommen und einer Behinderung aufgezeigt werden. Und auch in der Ereignisdatenanalyse, in der nur Personen, die zu Beginn keine Behinderung aufweisen, über mehrere Befragungswellen analysiert werden, zeigt sich, dass Probleme mit dem Einkommen auszukommen mit einer erhöhten Wahrscheinlichkeit eine Behinderung zu bekommen verknüpft sind. Dieser Zusammenhang wird jedoch mit zunehmendem Alter geringer.

\subsubsection{Subjektive Gesundheit}

Viele Studien, die den Zusammenhang von finanzieller Situation und Gesundheit untersuchen, tun dies anhand der von den Befragten berichteten subjekti- 
ven Gesundheit. Im Querschnitt zeigt sich in den vielen vorliegenden Forschungsarbeiten ein einheitliches Bild. So sind beispielsweise ein geringes Vermögen (Aittomäki et al. 2010) und Probleme mit dem verfügbaren Einkommen über die Runden zu kommen (z.B. Tucker-Seeley et al. 2013; Terraneo 2017; Ahnquist/Wamala/Lindstrom 2012; Angel et al. 2003) mit einer schlechteren Bewertung der eigenen Gesundheit verknüpft.

Mit den Daten einer Organisation, die Menschen mit finanziellen Schwierigkeiten hilft, liefern O'Neill et al. (2006) Hinweise darauf, dass die subjektive Gesundheit umso besser ist, je weniger ,negative finanzielle Ereignisse“ auftreten. Die oben erwähnte Studie von Adena und Myck (2014) untersucht auch Veränderungen in der subjektiven Gesundheit. Die Ergebnisse zeigen, dass subjektive Armut, Vermögensarmut und - im Gegensatz zu den anderen Gesundheitsindikatoren - auch Einkommensarmut zum ersten Zeitpunkt mit einer Verschlechterung der subjektiven Gesundheit bzw. einer unwahrscheinlicheren Verbesserung der Gesundheit einhergeht. Ahnquist et al. (2007) verwenden drei Beobachtungszeitpunkte der Swedish Survey of Living Conditions, um langfristige kumulative Effekte von finanziellen Schwierigkeiten auf die subjektive Gesundheit zu analysieren. Die Berechnungen zeigen, dass eine schlecht bewertete Gesundheit zum dritten Beobachtungszeitpunkt umso wahrscheinlicher ist, je häufigen von finanziellen Problemen bei den vorherigen Befragungen berichtet wurde.

Neben den zahlreichen Studien, die den Zusammenhang von finanzieller Situation und subjektiver Gesundheit mit den gängigen Querschnittsmethoden untersucht haben, gibt es inzwischen auch einige Analysen, die Panelmodelle verwenden, um den Einfluss von Veränderungen der finanziellen Situation auf Veränderungen der Gesundheit genauer zu untersuchen. Die meisten Studien, die den Zusammenhang von Einkommen und subjektiver Gesundheit im Längsschnitt untersuchen, finden einen geringen positiven Effekt des Einkommens auf die Gesundheit (Imlach Gunasekara/Carter/Blakely 2011). Wie auch bei den anderen Gesundheitsindikatoren sind Untersuchungen, die die finanzielle Situation nicht anhand von Einkommen messen, auch bei der subjektiven Gesundheit seltener (vgl. auch Georgiades et al. 2009).

Die Studie von Imlach Gunasekara et al. (2011) ist eine der wenigen, die eine Fixed-Effects-Analyse anwendet, um Veränderungen der subjektiven Gesundheit zu untersuchen. Anhand von drei, innerhalb von vier Jahren erhobenen, Befragungswellen des Survey of Family, Income and Employment aus Neuseeland kommen sie zu dem Ergebnis, dass das Leben in Deprivation mit schlechterer subjektiver Gesundheit verknüpft ist. Piumatti (2017) untersucht mit jährlich erhobenen Paneldaten von älteren Menschen den Zusammenhang von Veränderung in der finanziellen Situation - gemessen anhand der Schwierigkeit mit dem Einkommen über die Runden zu kommen - und der subjektiven Gesundheit. Auf Basis der Entwicklung der finanziellen Situation über vier Jahre wurden die Befragten in drei Gruppen eingeteilt. Etwa zwei Drittel der 
Befragten befindet sich in der Gruppe mit einer konstant guten finanziellen Situation. Die restlichen Befragten teilen sich ungefähr gleichmäßig in Gruppen auf mit einer konstant schlechten sowie einer zu Beginn mittleren, im Zeitverlauf schlechter werdenden finanziellen Situation. Bei der anschließenden Betrachtung der Entwicklung der subjektiven Gesundheit fällt auf, dass die beiden Gruppen mit konstanter finanzieller Situation über den Beobachtungszeitraum auch eine konstante Gesundheit aufweisen. Zwischen den beiden Gruppen besteht jedoch ein deutlicher Unterschied bei der Bewertung der Gesundheit zugunsten der Personen mit einer besseren finanziellen Situation. Für die Gruppe, die innerhalb der vier Jahre eine Verschlechterung der finanziellen Situation erlebt, verschlechtert sich die Gesundheit in dieser Zeit deutlich, in etwa vom Niveau der Gruppe mit wenig finanziellen Problemen auf das Niveau der Gruppe mit konstant größeren Problemen.

Anhand von drei Wellen des Sozioökonomischen Panels und RandomEffects-Analysen untersuchen Pfoertner et al. (2011), wie zwei unterschiedliche Maße für Armut - Einkommensarmut und inadäquater Lebensstandard mit der subjektiven Gesundheit verbunden sind. Einkommensarmut ist dabei definiert als ein Äquivalenzhaushaltseinkommen von weniger als 50\% des Medianeinkommens. Der Lebensstandard wird als inadäquat betrachtet, wenn drei oder mehr von elf Kriterien aufgrund finanzieller Gründe nicht zutreffen. Dazu gehört u.a. die Verfügbarkeit eines Fernsehers, Autos und Telefons, eine adäquate Wohnung und die Möglichkeit für Notfälle zu sparen. Die Ergebnisse zeigen, dass beide Armutsindikatoren mit schlechter Gesundheit verknüpft sind. Der Einfluss eines inadäquaten Lebensstandards ist jedoch stärker als der Einfluss von Einkommensarmut. In Berechnungen, die beide Maße als unabhängige Variable enthalten, wird für Einkommensarmut kein Effekt gefunden, im Gegensatz dazu bleibt der signifikante Effekt des Lebensstandards bestehen. Ein weiteres Ergebnis der Studie ist, dass die Wahrscheinlichkeit schlechter Gesundheit kontinuierlich ansteigt, je länger eine Person mit einem inadäquaten Lebensstandard lebt. Auch eine Studie aus Großbritannien kommt anhand von Random-Effects-Modellen und Längsschnittdaten aus zwei großen Studien zu dem Ergebnis, dass finanzielle Schwierigkeiten mit einer schlechten subjektiven Gesundheit verknüpft sind (French 2018).

\subsubsection{Vergleich von Quer- und Längsschnittanalysen}

Betrachtet man die bisherige Forschung, so fällt auf, dass die Studien, die den Zusammenhang von finanzieller Situation und Gesundheit im Längsschnitt betrachtet haben, zu weniger übereinstimmenden Ergebnissen kommen wie die zahlreichen Querschnittsanalysen. Es existieren einige Studien, die mit denselben Daten Quer- und Längsschnittberechnungen durchführen. Diese kommen alle zu dem Ergebnis, dass in den Längsschnittanalysen die Veränderung der 
Gesundheit deutlich schwächer, in manchen Fällen auch überhaupt nicht mehr, von der finanziellen Situation abhängt, als es in den Querschnittsanalysen den Anschein hat. Gezeigt wurde dies für die subjektive Gesundheit (Überblick bei Imlach Gunasekara/Carter/Blakely 2011; siehe auch Imlach Gunasekara et al. 2013), Depression (Heflin/Iceland 2009; Bridges/Disney 2010; Franzese 2015; Frijters/Ulker 2008; Jones/Wildman 2008; Zimmerman/Katon 2005) und Einschränkungen in Aktivitäten des täglichen Lebens (Frijters/Ulker 2008). Diese Ergebnisse lassen darauf schließen, dass die Zusammenhänge, die im Querschnitt sichtbar sind, zum Großteil auf individuelle (unveränderliche) Eigenschaften zurückzuführen sind, die in den Querschnittsmodellen nicht berücksichtigt werden (siehe Kapitel 4.6).

\subsection{Verbesserung und Verschlechterung der finanziellen Situation}

Der Forschungsstand zu der Frage nach unterschiedlichen Effektstärken bei Verbesserung und Verschlechterung ist sehr übersichtlich. Diese Frage wurde bisher vor allem anhand von mentaler Gesundheit erforscht. Die Studien von Lorant et al. (2007) sowie McKenzie et al. (2014) sind die bislang aussagekräftigsten, da sie sich vor allem methodisch (Fixed-Effects-Modelle) aber auch durch die verwendeten Daten (mehr als zwei Zeitpunkten) von den anderen Untersuchungen abheben.

Lorant et al. (2007) verwenden Daten von acht Wellen (1992-1999) des Belgian Household Panel Survey, um den Zusammenhang von sozioökonomischen Faktoren und Depression zu untersuchen. Depression wurde hierbei mit einer Skala von 0 bis 72 gemessen, die aus den Antworten von 18 Fragen resultiert. Als sozioökonomische Indikatoren wurden das Äquivalenzhaushaltseinkommen, Deprivation, Einkommensarmut und Probleme mit dem verfügbaren Einkommen auszukommen verwendet. Bivariate Fixed-Effects-Regressionen deuten darauf hin, dass finanzielle Probleme, Einkommensarmut und Deprivation mit einem erhöhten Wert auf der Depressionsskala verknüpft sind. Im zweiten Schritt der Analyse wurde die Richtung der Veränderungen in den ökonomischen Variablen betrachtet. Im Vergleich zu keiner Veränderung, kann bei einer Verschlechterung der finanziellen Situation höhere und bei einer Verbesserung der finanziellen Situation geringere Werte auf der Depressionsskala beobachtet werden. Die Effektstärke ist bei Verschlechterung größer als bei einer Verbesserung der finanziellen Lage, wobei zwischen den Koeffizienten jedoch nur für Probleme mit dem Einkommen auszukommen ein signifikanter Unterschied besteht.

Auch Mckenzie et al. (2014) verwenden Längsschnittdaten, um den Einfluss von Armutsindikatoren auf die mentale Gesundheit zu untersuchen. Sie 
analysieren drei Beobachtungszeitpunkte des New Zealand Survey of Family, Income and Employment. Als Maße für die finanzielle Lage werden der Erwerbsstatus, das Haushaltseinkommen sowie individuelle Deprivation und Deprivation auf regionaler Ebene betrachtet. Um die mentale Gesundheit zu messen, kommen zwei verschiedene Maße zum Einsatz. Ein Index basierend auf fünf Fragen zu positiven und negativen Gefühlen zur Messung der allgemeinen mentalen Gesundheit sowie die Kessler-10-Skala, die anhand von zehn Fragen zu negativen Gefühlen den psychologischen Stress abbildet (ebd.: 254). Die Ergebnisse der Fixed-Effects-Analysen zeigen keine signifikanten Zusammenhänge von Arbeitslosigkeit, Haushaltseinkommen und Deprivation auf regionaler Ebene mit den beiden Skalen für mentale Gesundheit. Individuelle Deprivation ist hingegen mit schlechter mentaler Gesundheit und psychologischem Stress verknüpft. Im nächsten Schritt wird für individuelle Deprivation ein First-Difference-Modell berechnet, wobei Variablen für keine Veränderung, Verbesserung und Verschlechterung von jeweils ein, zwei und drei oder mehr Einheiten verwendet werden. Diese Berechnungen zeigen, dass die $\mathrm{Zu}-$ sammenhänge von Deprivation und den beiden Indikatoren mentaler Gesundheit nicht linear sind, d.h., dass z.B. eine Veränderung um zwei Einheiten im First-Difference-Modell eine größere Veränderung in der mentalen Gesundheit zur Folge hat, als es das lineare Modell vermuten lässt. Zudem wird getestet, ob die Effektstärken von den entsprechenden positiven und negativen Veränderungen (z.B. Verbesserung um eine Einheit im Vergleich zur Verschlechterung um eine Einheit) unterschiedlich sind. Hierbei können jedoch keine signifikanten Unterschiede festgestellt werden.

Eine Studie von Mirowsky und Ross (2001) untersucht auch die Veränderung der finanziellen Situation zwischen den beiden Untersuchungszeitpunkten. Die Betrachtung der Veränderung der depressiven Symptome kommt zu dem Ergebnis, dass finanzielle Probleme zum zweiten Zeitpunkt - unabhängig vom ersten Zeitpunkt - mit mehr depressiven Symptomen einhergehen. Dieser Effekt wird mit zunehmendem Alter geringer. Das Überwinden der finanziellen Probleme (Probleme zum ersten, aber nicht mehr bei der zweiten Befragung) scheint nur bei jüngeren Altersgruppen mit einem geringen Rückgang der depressiven Symptome verknüpft zu sein.

Mit Daten aus zwei Wellen der Australian Personality and Total Health (PATH) Through Life Study untersuchen Butterworth et al. (2009) den Zusammenhang von finanziellen Schwierigkeiten und Depression. Als abhängige Variable wird ein binärer Indikator für Depression verwendet, wobei eine Person bei einem Wert von mindestens 7 auf der Goldberg-Depressionsskala als depressiv eingestuft wird (mögliche Werte 0-9). Eine Schwäche dieser Studie ist, dass finanzielle Schwierigkeiten in den beiden Wellen nicht in der gleichen Weise erhoben wurden. Das geringste Risiko einer Depression kann für die Befragten beobachtet werden, die zu keinem Zeitpunkt finanzielle Probleme 
hatten. Eine Verschlechterung der finanziellen Situation ist mit erhöhtem Depressionsrisiko verbunden. Denn die Berechnungen zeigen, dass aktuelle finanzielle Probleme mit einer höheren Wahrscheinlichkeit depressiv zu sein verbunden sind, unabhängig von der finanziellen Situation bei der ersten Befragung. Im Vergleich dazu haben Personen, die finanzielle Schwierigkeiten in der ersten Welle, aber keine Schwierigkeiten in der zweiten Welle hatten, ein geringeres Depressionsrisiko. Das deutet darauf hin, dass durch die Verbesserung der finanziellen Situation das Risiko einer Depression gesenkt wird.

Sareen et al. (2011) analysieren die mentale Gesundheit im Zusammenhang mit Veränderungen im Haushaltseinkommen zwischen zwei Zeitpunkten mit einem Abstand von drei Jahren. Hierbei wird zwischen drei Gruppen unterschieden: verringertes, konstantes und steigendes Einkommen. Die Berechnungen zeigen, dass eine Verschlechterung der finanziellen Lage - im Vergleich zu keiner Veränderung - die Wahrscheinlichkeit an einer psychischen Krankheit zu leiden erhöht. Bei einem Anstieg des Einkommens kann hingegen kein Unterschied zur Gruppe des konstanten Einkommens festgestellt werden.

Im Rahmen der Forschung zu sozialer Mobilität untersuchen Dolan und Lordan (2013) Auf- und Abstiege der finanziellen Position zwischen der Kindheit und dem Erwachsenenalter. Veränderungen in der relativen Einkommensposition sind in den Analysen mit mentaler Gesundheit und Lebenszufriedenheit verknüpft. Zudem stimmen die Ergebnisse mit der Hypothese der Verlustaversion überein: Abstieg hat einen größeren Einfluss als Aufstieg.

Es existieren nur sehr wenige Studien, die Veränderungen in der ökonomischen Situation nach Verbesserung und Verschlechterung aufteilen und in $\mathrm{Zu}$ sammenhang mit physischer Gesundheit betrachten. Piumatti $(2017)^{7}$ stellt fest, dass eine Verschlechterung der subjektiven finanziellen Situation mit schlechter werdender subjektiver Gesundheit einhergeht. Es kann keine Aussage darüber gemacht werden, wie sich eine bessere finanzielle Lage auf die Gesundheit auswirkt, da die verfügbaren Fallzahlen dieser Situation zu gering waren (ebd.: 326). Giordano und Lindstrom (2010) untersuchen die Veränderung der subjektiven Gesundheit zwischen zwei Zeitpunkten mit einem Abstand von 6 Jahren in Abhängigkeit von der Veränderung des Einkommens. In den bivariaten Analysen geht das Aufsteigen aus dem untersten in ein höheres Einkommensquartil mit einer höheren Wahrscheinlichkeit von guter Gesundheit einher. Allerdings ist dieser Zusammenhang unter Kontrolle weiterer Faktoren nicht mehr signifikant. Ein Abrutschen in das unterste Einkommensquartil ist hingegen auch in multivariaten Berechnungen mit einer höheren Wahrscheinlichkeit von schlechter Gesundheit verknüpft.

Die Frage, ob negative und positive Veränderungen der finanziellen Lage einen Einfluss auf die Gesundheit haben, wurde in den dargestellten Studien

Die Studie wurde bereits im vorangegangenen Kapitel näher beschrieben. 
anhand von verschiedenen Maßen für die finanzielle Situation und Gesundheit behandelt. Die Ergebnisse stimmen nicht vollständig überein, dennoch ist eine Tendenz erkennbar. Alle Analysen können negative Effekte auf die Gesundheit bei einer Verschlechterung der finanziellen Situation aufzeigen. Zudem wird meistens ein schwächerer oder gar kein Zusammenhang von Gesundheit und verbesserter finanzieller Lage gefunden. Die bisherige Forschung spricht also für die Hypothese der Verlustaversion.

\subsection{Einkommens- und Vermögensungleichheit und Gesundheit}

Es gibt vereinzelt Studien (z.B. Qi 2012), die von Hinweisen berichten, dass der sichtbare Zusammenhang von Ungleichheit und Gesundheit nur ein statistisches Artefakt ist (wie in Kapitel 2.2.8 beschrieben). Trotz dieser Ergebnisse - und obwohl die Ergebnisse der vielen Studien nicht vollständig übereinstimmen - herrscht weitgehend Konsens, dass es sich bei dem Zusammenhang nicht um ein Artefakt handelt (Wilkinson/Pickett 2006, 2009b; siehe auch Layte/Whelan 2014; Auger et al. 2012).

In den folgenden Abschnitten wird der Forschungsstand zum Zusammenhang von Einkommensungleichheit und Gesundheit dargestellt. Zuerst wird ein kurzer Überblick über Analysen gegeben, die dieses Thema auf der Makroebene betrachten, anschließend folgt eine Zusammenfassung von Studien, die Individualdaten analysieren. Es wird jeweils der Forschungsstand zu verschiedenen Gesundheitsindikatoren dargestellt, wobei der Fokus auf den in dieser Arbeit verwendeten Maßen liegt: Depression, Einschränkungen in Aktivitäten und subjektive Gesundheit. Im Anschluss wird auf eine mögliche Zeitverzögerung der Wirkung der Ungleichheit eingegangen und die bekannten Forschungsergebnisse berichtet. Weiterhin wird der Forschungsstand dargestellt bezüglich der Frage, ob die ganze Bevölkerung oder nur bestimmte Gruppen unter der Ungleichverteilung des Einkommens leiden. Darauf folgt ein Überblick über die bisherigen Studien, die die psychosoziale und neomaterielle Theorie überprüft haben. Zum Abschluss des Kapitels wird der - kaum vorhandene - Stand der Forschung zu den gesundheitlichen Auswirkungen der Vermögensungleichheit beschrieben.

\subsubsection{Makrostudien}

Bei den Studien, die den Zusammenhang von Einkommensungleichheit und physischer Gesundheit anhand von Makroindikatoren untersuchen, wird überwiegend der vermutete Zusammenhang gefunden (Überblick bei Torre/ 
Myrskylä 2014). Am häufigsten wurde dabei Mortalität als abhängige Variable verwendet. Es gibt jedoch auch Analysen anderer Gesundheitsmaße wie z.B. Übergewicht (Pickett et al. 2005) und Greifkraft (Vries/Blane/Netuveli 2014). Es scheint dabei methodische Gründe zu haben, warum einige Untersuchungen keinen Zusammenhang feststellen konnten: zu kleine Untersuchungseinheiten (z.B. Regionen oder Stadtteile), eine Stichprobe mit homogenen Ländern oder die Verwendung von Panelanalysen, bei denen für unbeobachtete Heterogenität kontrolliert wird (Torre/Myrskylä 2014; Wilkinson/Pickett 2006; Hu/van Lenthe/Mackenbach 2015). Zahlenmäßig sind die Querschnittsstudien, die eine Korrelation feststellen, deutlich in der Überzahl. Allerdings ist der $\mathrm{Zu}$ sammenhang in den wenigen Längsschnittstudien längst nicht so eindeutig wie Pickett und Wilkinson es darstellen (Pickett/Wilkinson 2015). Die Studie von Hill und Jorgenson (2018) bietet einen Überblick über bisherige Untersuchungen, die mit Fixed-Effects-Analysen den Einfluss von Einkommensungleichheit auf Lebenserwartung und Mortalität untersucht haben. Hierbei sind in mehr als der Hälfte der Studien keine Zusammenhänge zu erkennen.

Viele Studien belegen einen (starken) Makrozusammenhang von Einkommensungleichheit und mentalen Erkrankungen (Wilkinson/Pickett 2017; Pickett/Wilkinson 2010; Pickett/James/Wilkinson 2006). Untersucht wurde bisher u.a. Schizophrenie (Burns/Tomita/Kapadia 2014; Boydell et al. 2004), psychotische Symptome (Johnson/Wibbels/Wilkinson 2015), Angststörungen (Pickett/Wilkinson 2010) und allgemeine Indikatoren mentaler Gesundheit (Layte 2012). Auch Depression wurde in diesem Zusammenhang untersucht, wobei ebenfalls signifikante Zusammenhänge gefunden wurden (van Deurzen/ van Ingen/van Oorschot, Wim J. H. 2015; Messias/Eaton/Grooms 2011).

\subsubsection{Individualebene}

Neben den Studien, die lediglich Makroindikatoren untersuchen, gibt es eine Reihe an Studien, die Individualdaten verwenden und somit sowohl auf Makroindikatoren, als auch auf individuelle Eigenschaften kontrollieren können. Eine Metaanalyse von Mehrebenenanalysen kommt zu dem Ergebnis, dass das Mortalitätsrisiko in Regionen mit hoher Einkommensungleichheit erhöht ist (Kondo et al. 2009). Auch die Untersuchung von Maio (2008) bestätigt anhand von Daten aus Argentinien einen Zusammenhang von Ungleichheit und Lebenserwartung. Allerdings ist die Ungleichheit nicht korreliert mit anderen untersuchten Gesundheitsindikatoren. Maio leitet draus ab, dass - im Gegensatz zur gängigen Praxis - Mortalität und Morbidität nicht als austauschbare Maße verwendet werden können (ebd.). Dies zeigen auch Studien, die verschiedene Aspekte von Gesundheit untersuchen und weniger deutliche Effekte beschreiben als die Literatur zu Mortalität (siehe z.B. Anderson/Bjorklund/Rambotti 2017). Es konnte beispielsweise gezeigt werden, dass in den USA das Risiko 
eines Herzinfarktes in Bundesstaaten mit größerer Ungleichheit erhöht ist (Pabayo/Kawachi/Gilman 2015). Ein Zusammenhang von Einkommensungleichheit der US-Bundesstaaten und hohem Blutdruck sowie Körpergewicht konnte jedoch nur bei Frauen beobachtet werden (Diez-Roux/Link/Northridge 2000). Basierend auf kolumbianischen Daten berichten auch Lucumi et al. (2017) von negativen Auswirkungen für den Blutdruck ausschließlich für Frauen. Uneinheitliche Ergebnisse liefern die bisherigen Studien zur Gesundheit von Zahnfleisch und Zähnen (Singh et al. 2018; Bernabé/Marcenes 2011; Sun et al. 2017).

Es gibt Studien, die verschiedene Indikatoren für mentale Gesundheit im Zusammenhang mit Einkommensungleichheit auf verschiedenen Ebenen betrachten und dabei zu uneinheitlichen Ergebnissen kommen. Sowohl in Nachbarschaften in Schweden (Lofors/Ramírez-León/Sundquist 2006) als auch im Großraum São Paulo (Chiavegatto Filho, Alexandre Dias Porto et al. 2013) sowie bei der Analyse von 60 Metropolregionen in den USA (Sturm/Gresenz 2002) konnte kein Zusammenhang von Einkommensungleichheit und Angststörungen festgestellt werden. Im Gegensatz dazu stehen Studien, die eine Rolle der Einkommensungleichheit für das Auftreten einer Psychose nahelegen (Burns/Esterhuizen 2008; Kirkbride et al. 2014). Eine Analyse von Einkommensungleichheit auf Länderebene kommt zu dem Ergebnis, dass eine größere Ungleichheit mit schlechterem mentalem Wohlbefinden verknüpft ist (Layte 2012). In einem Überblick über die Literatur zu Einkommensungleichheit und mentaler Gesundheit werden gemischte Ergebnisse berichtet: Neun Studien finden einen Zusammenhang, zehn Studien bestätigen einen Zusammenhang nur für bestimmte Gruppen und in acht Studien wird kein Zusammenhang gefunden (Ribeiro et al. 2017). Das Ergebnis der anschließenden Metaanalyse, in der nur neun der 27 Analysen berücksichtigt werden konnten, bestätigt einen schwachen Zusammenhang von Ungleichheit und mentaler Gesundheit. Außerdem konnte kein Muster erkannt werden, das die Heterogenität zwischen den Studien erklärt. Weder die Stichprobengröße, noch die Ebene der Ungleichheitsmessung, die Methode der Messung der mentalen Gesundheit, die Bewertung der Qualität der Studie oder Verwendung von individuellem Einkommen als Kontrollvariable erklären die uneinheitlichen Ergebnisse der Studien (ebd.).

Für Depression - der Indikator von mentaler Gesundheit, der in dieser Arbeit untersucht wird - wird die Theorie des schädlichen Einflusses von großer Ungleichheit überwiegend bestätigt (z.B. van Deurzen/van Ingen/van Oorschot, Wim J. H. 2015; Pabayo/Kawachi/Gilman 2014; Melar/Rossi 2012; Cifuentes et al. 2008; Muramatsu 2003; Fiscella/Franks 2000; Kahn et al. 2000). Wie in dem Übersichtsartikel von Patel et al. (2018) deutlich wird, berichten fast zwei Drittel der Studien - und fünf von sechs Längsschnittstudien - einen signifikant positiven Zusammenhang zwischen Einkommensungleichheit und Depression. Hierbei scheint die Ebene, auf der Ungleichheit gemessen wird, 
keine Rolle zu spielen. Auf allen untersuchten Ebenen - lokal, regional, national - werden signifikante Zusammenhänge gefunden.

Die subjektive Gesundheit ist auch in der Literatur zu Einkommensungleichheit ein oft verwendetes Maß. Signifikante Zusammenhänge von schlechterer subjektiver Gesundheit bei mehr Ungleichheit werden von einigen Studien berichtet (Kennedy et al. 1998; Adeline/Delattre 2017; Präg/Mills/Wittek 2014; Hildebrand/van Kerm 2009; Kondo et al. 2009; Rajan/Kennedy/King 2013; Subramanian/Kawachi/Kennedy 2001; Subramanian/Kawachi 2006; Fiscella/Franks 2000; Kondo et al. 2012). Es gibt jedoch auch Analysen, die keine Korrelation aufzeigen (Qi 2012; Maio 2008; Ding/Billari/Gietel-Basten 2017; Olsen/Dahl 2007; Arezzo 2018). Auch Subramanian/Kawachi (2004) stellen in ihrer Übersicht von Studien, die sich diesem Thema widmen und bis 2004 veröffentlicht wurden, uneinheitliche Ergebnisse fest. Karlsson et al. (2010) finden in ihren Analysen nur in Ländern mit hohem Einkommen einen Zusammenhang von Ungleichheit und subjektiver Gesundheit.

Studien, die körperliche Einschränkungen bei der Ausführung von Aktivitäten untersuchen, sind ebenfalls durch uneinheitliche Forschungsergebnisse gekennzeichnet. In Studien mit chinesischen (Li/Zhu 2006) sowie US-amerikanischen Daten (Fuller-Thomson/Gadalla 2008; Gadalla/Fuller-Thomson 2008; Montez/Hayward/Wolf 2017) ${ }^{8}$ konnten signifikante Effekte von Einkommensungleichheit auf die Einschränkungen bei Aktivitäten festgestellt werden. In einer argentinischen Untersuchung (Maio 2008) sowie in einer Studie, die Einkommensungleichheit auf der Ebene von Nationalstaaten untersucht (Karlsson et al. 2010), ist hingegen kein Zusammenhang mit Einschränkungen in Aktivitäten festzustellen. Vries/Blane/Netuveli (2014) untersuchen die USA und europäische Länder, um die Hypothese der Einkommensungleichheit mit Daten der älteren Bevölkerung mit verschiedenen Gesundheitsindikatoren zu testen. Während ein Einfluss von Ungleichheit auf objektive Maße (Greifkraft und exspiratorischer Atemstrom) bestätigt wird, ist dies für die selbst berichteten Einschränkungen nicht der Fall. Allerdings belegt die Studie, dass eine im Zeitverlauf steigende Ungleichheit mit mehr Einschränkungen verknüpft ist (ebd.). Anhand von europäischen Daten analysieren Brandt/Deindl/Hank (2012) ,successful ageing“, das u.a. durch geringe körperliche Einschränkungen gekennzeichnet ist. Sie kommen zu dem Ergebnis, dass größere Ungleichheit mit einer geringeren Wahrscheinlichkeit von ,successful ageing" verbunden ist.

Die stärksten Effekte von Ungleichheit wurden festgestellt bei der Untersuchung von Kindersterblichkeit und der Gesundheit von jungen Menschen

Alle diese Analysen verwenden Daten der gleichen Umfrage, des American Community Survey, allerdings aus verschiedenen Jahren: (Fuller-Thomson/Gadalla 2008) verwenden Daten aus 2003, (Gadalla/Fuller-Thomson 2008) nutzen Daten aus 2006 und (Montez/Hayward/Wolf 2017) analysieren die Jahre 2010-2014. 
(vgl. Rözer/Volker 2016; Vries/Blane/Netuveli 2014). Die Hypothesen anhand von älteren Menschen zu testen ist jedoch von besonderer Bedeutung, da sie deutlich mehr Krankheiten und höhere Mortalitätsraten haben als die jüngere Bevölkerung und daher die gesundheitlichen Unterschiede zwischen Ländern stark beeinflussen (Vries/Blane/Netuveli 2014). Es existieren einige Studien, die den Zusammenhang von Einkommensungleichheit und Gesundheit für die ältere Bevölkerung angeschaut haben (Feng et al. 2012; Vries/Blane/Netuveli 2014; Ding/Billari/Gietel-Basten 2017). Vor allem die Studien, die Depression untersuchen, kommen dabei zu sehr unterschiedlichen Ergebnissen (Muramatsu 2003; Ladin/Daniels/Kawachi 2010; Choi et al. 2015; Fernández-Niño et al. 2014; Marshall et al. 2014). Diese Studien basieren in der Regel auf Querschnittsdaten und berücksichtigen nur die aktuelle Ungleichheit. Vermutlich spielen jedoch auch, oder vor allem, die Bedingungen im früheren Leben eine Rolle. Die älteren Menschen haben im Laufe ihres Lebens verschiedene Niveaus und Veränderungen in der Ungleichheit erlebt. Um dies zu berücksichtigen, analysieren Vries/Blane/Netuveli (2014) die Ungleichheit über einen langen Zeitraum, wobei sie einen Zusammenhang mit der Gesundheit feststellen können.

\subsubsection{Zeitverzögerte Messung der Ungleichheit}

Eine weitere Möglichkeit zur Berücksichtigung von früheren Erfahrungen ist die zeitversetzte Messung der Indikatoren. Zudem ist die zeitliche Verzögerung mit der die Einkommensungleichheit wirkt ein Aspekt, der angeführt wird, um widersprüchliche Forschungsergebnisse $\mathrm{zu}$ erklären (siehe Avendano/Hessel 2015). Die meisten Studien verknüpfen Daten zu Einkommensungleichheit und Gesundheit, die zum selben Zeitpunkt erhoben wurden. Es ist jedoch plausibel, dass Änderungen in der Ungleichheit einer Gesellschaft nicht sofortige Effekte nach sich ziehen, sondern eher erst durch längere Exposition und mit zeitlicher Verzögerung wirken. Es existieren einige Studien, die dieser Vorstellung Rechnung tragen und Ungleichheit zu einem früheren Zeitpunkt mit der Gesundheit zu einem späteren Zeitpunkt in Verbindung setzen.

Ein Ansatz ist hierbei die Betrachtung der Ungleichheit während der Kindheit. Umwelteinflüsse können in der Wachstumsphase des Körpers einen besonders starken Effekt auf die Gesundheit haben, da die Entwicklung des Gehirns und anderer Organe gestört und somit dauerhaft beschädigt werden kann (Dragano/Siegrist 2006; siehe auch Kapitel 2.2.5). In einer Auswertung von Daten von Jugendlichen aus Europa und Nordamerika wurde ein positiver $\mathrm{Zu}$ sammenhang zwischen Einkommensungleichheit in den ersten Lebensjahren und psychosomatischen Symptomen in der Jugend festgestellt (Elgar et al. 
2017). Zwei Studien zeigen für die USA einen Zusammenhang von Ungleichheit in der Kindheit mit der Gesundheit im Erwachsenenalter (Burkhauser et al. 2016; Lillard et al. 2015). Für Großbritannien wird dieser Zusammenhang zwar beobachtet, allerdings erweist er sich in multivariaten Analysen unter Kontrolle von anderen Einflussfaktoren nicht als robust und nicht signifikant (Burkhauser et al. 2016).

Inzwischen lassen sich auch einige Studien finden, die eine andere Herangehensweise verfolgen. Hierbei wird durch das Testen von verschiedenen zeitlichen Verzögerungen ermittelt, wann der stärkste Zusammenhang zu finden ist. (Hu/van Lenthe/Mackenbach 2015) untersuchten Mortalität und haben mit bis zu zehn Jahren Verzögerung keine signifikanten Zusammenhänge entdeckt. (Mellor/Milyo 2003) testen 10 sowie 20 Jahre Verzögerung und haben dabei für die Sterblichkeit aufgrund von kardiovaskulären Krankheiten und Tumorerkrankungen, aber nicht für allgemeine Mortalität, einen Zusammenhang gefunden. Eine andere Studie beschreibt eine Korrelation von Ungleichheit und Mortalität bei einem zeitlichen Abstand von fünf bis zwölf Jahren, wobei das Maximum bei sieben Jahren liegt (Zheng 2012). Für Einschränkungen in der Mobilität wurde der stärkste Zusammenhang bei einem Zeitabstand von 20 Jahren berichtet (Gadalla/Fuller-Thomson 2008), in zwei Analysen subjektiver Gesundheit ist es mit 15 Jahren etwas weniger (Blakely 2000; Subramanian/Kawachi 2004). Auch eine Metaanalyse kam zu dem Ergebnis, dass Studien, die Gesundheit und Ungleichheit zeitversetzt gemessen haben, stärkere Zusammenhänge berichten (Kondo et al. 2012). Jedoch ist auch ein Ergebnis bisheriger Forschung, dass die Gesundheit und die Ungleichheit bei gleichzeitiger Messung signifikant miteinander verknüpft sind, auch wenn auf frühere Ungleichheit kontrolliert wird (Subramanian/Kawachi 2006). (Glymour 2008) stellt zudem fest, dass eine unangemessen spezifizierte zeitliche Verzögerung der Einkommensungleichheit die Ergebnisse von Längsschnittberechnungen verzerren kann und sogar eine Umkehrung des Vorzeichens möglich ist.

\subsubsection{Wer leidet unter der Ungleichheit?}

Die Frage, ob alle oder nur bestimmte Gruppen in einer Gesellschaft von negativen Effekten der Ungleichheit betroffen sind, wird theoretisch diskutiert (siehe Kapitel 2.2.8), aber auch in vielen Studien empirisch getestet. Der Stand der Forschung ist in diesem Punkt uneinheitlich. Manche Untersuchungen stützen die schwache Hypothese, dass nur bei der unteren (Einkommens-) Schicht die Auswirkungen von Ungleichheit zu beobachten sind (z.B. Kahn et al. 2000; Wagstaff/van Doorslaer 2000). Andere Studien stützen hingegen die starke Hypothese, dass alle Bevölkerungsschichten von der Ungleichheit beeinflusst werden. (z.B. Subramanian/Kawachi/Kennedy 2001; Subramanian/Kawachi 
2006; Adeline/Delattre 2017; Hildebrand/van Kerm 2009). Wilkinson und Pickett $(2008 ; 2009 b)$ fassen die Ergebnisse folgendermaßen zusammen: Alle profitieren von größerer Gleichheit, aber die Armen etwas mehr als die Reichen. Zum Teil werden jedoch auch Ergebnisse berichtet, die nicht in dieses Muster passen. Eine Studie, die den Zusammenhang von Einkommensungleichheit und Depression in Europa untersucht, legt nahe, dass der negative Effekt von hoher Ungleichheit am stärksten die mittlere Schicht trifft (van Deurzen/van Ingen/van Oorschot, Wim J. H. 2015). Weich/Lewis/Jenkins (2001) kommen bei der Analyse von Daten aus Großbritannien zu dem Ergebnis, dass der Zusammenhang von Einkommensungleichheit und mentaler Gesundheit mit dem individuellen Einkommen variiert. Bei Personen mit hohem Einkommen ist die mentale Gesundheit in Regionen mit hoher Ungleichheit schlechter als in Regionen mit geringerer Ungleichheit. In der unteren Einkommensgruppe ist es jedoch umgekehrt. Bei höherer Ungleichheit ist die mentale Gesundheit besser als bei geringer Ungleichheit.

\subsubsection{Kausale Effekte?}

Wilkinson und Pickett sehen einen Zusammenhang zwischen Ungleichheit und Gesundheit als bewiesen und einen kausalen Effekt als wahrscheinlich an (Wilkinson/Pickett 2006; Pickett/Wilkinson 2015). Sie verweisen auf die große Anzahl an Studien in diesem Bereich und argumentieren, dass die entscheidenden Kriterien eines kausalen Zusammenhangs erfüllt sind (Pickett/Wilkinson 2015). Allerdings stehen wie oben beschrieben nicht alle Forschungsergebnisse im Einklang mit der Theorie. Die Studien, deren Ergebnisse nicht ins Bild passen, erklären Pickett \& Wilkinson folgendermaßen: „Of the small minority of studies which find no association, most can be explained by income inequality being measured at an inappropriate scale, the inclusion of mediating variables as controls, the use of subjective rather than objective measures of health, or follow up periods which are too short" (ebd.: 323).

Im Gegensatz zu Wilkinson und Pickett, fragen (Avendano/Hessel 2015), ob die Theorie verworfen werden muss. Auch an anderer Stelle wurde der (kausale) Effekt von Einkommensungleichheit angezweifelt (Lynch et al. 2004; Coburn 2015). Denn die Analysen, die die Theorie untermauern, sind zumeist reine Makroanalysen, die zweierlei methodische Probleme aufweisen. Erstens hängen die Ergebnisse stark von der Auswahl der Stichprobe ab. Pop et al. (2013) zeigen, dass sich der Zusammenhang von Einkommensungleichheit und Lebenserwartung in Ländern mit hohem Einkommen ändert, je nachdem wie viele und welche Länder berücksichtigt werden. Zweitens verwenden die meisten dieser Studien Daten im Querschnitt und berücksichtigen keine anderen Merkmale der untersuchten Länder, Regionen oder Nachbarschaften. 
Unterschiede bspw. im Gesundheitssystem, der Bevölkerungsstruktur, gruppenspezifischen Gesundheitsverhalten und Umweltbedingungen werden in diesen Analysen nicht beachtet. Die unbeobachtete Heterogenität in diesen Merkmalen könnte jedoch durch Fixed-Effects-Modelle aufgefangen werden, um präzisere Analyseergebnisse zu erhalten (Beckfield 2004). (Kondo et al. 2012) zeigen für subjektive Gesundheit in einer Metaanalyse, dass der Zusammenhang von Einkommensungleichheit und Gesundheit unter Kontrolle des durchschnittlichen Einkommen der untersuchten Gebiete signifikant schwächer ausfällt.

Auch bei Studien, die auf Individualdaten basieren, macht es einen Unterschied, welche Methoden verwendet werden. Lange Zeit waren auch hier die meisten Studien im Querschnitt (Vries/Blane/Netuveli 2014), doch seit einiger Zeit kommen nach und nach immer mehr Analysen von Längsschnittdaten hinzu. Neuere Studien in der Literatur zu Mortalität zeigen, dass die Verwendung von Paneldaten und -methoden die Ergebnisse beeinflussen. Einige Untersuchungen zeigen, dass der in Querschnittsberechnungen oft gezeigte negative Zusammenhang von Einkommensungleichheit und Gesundheit schwächer wird, verschwindet oder sich sogar umdreht, wenn auf zusätzliche Eigenschaften - im besten Fall durch die Fixed-Effects-Methode die unbeobachtete Heterogenität - kontrolliert wird (Hu/van Lenthe/Mackenbach 2015; Pop/van Ingen/van Oorschot 2013; Siddiqi/Jones/Erwin 2015; Clough-Gorr/Egger/Spoerri 2015; Beckfield 2004; Regidor et al. 2015; Biggs et al. 2010; Mellor/Milyo 2001). Vereinzelt konnte jedoch auch unter Anwendung von Fixed-EffectsModellen zumindest teilweise ein Zusammenhang von Einkommensungleichheit und Mortalität gefunden werden (Hill/Jorgenson 2018; Rasella/Aquino/Barreto 2013; Rebeira et al. 2017).

Auch bei der Analyse von mentaler (Bechtel/Lordan/Rao 2012; Medeiros 2017) und subjektiver Gesundheit (Lorgelly/Lindley 2008; Latif 2015) wurde aufgezeigt, dass die negativen Auswirkungen von Ungleichheit in Längsschnittstudien nicht bestätigt werden. (Hildebrand/van Kerm 2009) finden zwar einen signifikanten Zusammenhang von Einkommensungleichheit auf die subjektive Gesundheit, die Stärke dieses Effektes ist jedoch sehr gering. Welcher Bedeutung die Wahl der Kontrollvariablen und der Analysemethode hat, zeigt eine Studie, die verschiedene Methoden vergleicht (Kragten/Rözer 2017). Unter Kontrolle von sozialem Vertrauen, Wirtschaftskraft (BIP), Durchschnittsalter und einem Index für Humankapital ist der Zusammenhang im Querschnittsmodell anders als erwartet: Größere Ungleichheit korreliert mit besserer Gesundheit. Der Effekt dreht sich unter Verwendung eines FixedEffects-Modells jedoch wieder um. Die genannten Studien zeigen, dass unbeobachtete Heterogenität eine Rolle spielt und somit einfache Korrelationen im Querschnitt vorsichtig interpretiert werden sollten.

Berechnungen mit Paneldaten sind eine gute Methode, um sich dem kausalen Effekt anzunähern. Noch besser wären jedoch Experimente, bei denen 
man beobachten kann, wie Personen auf zufällig zugewiesene Ungleichheiten reagieren. Solche Experimente sind selbstverständlich nicht durchführbar. (Grönqvist/Johansson/Niknami 2012) kommen in ihrer Studie dieser Methode jedoch sehr nahe. Sie können den kausalen Zusammenhang von Einkommensungleichheit und Gesundheit in Schweden untersuchen. Da Flüchtlinge nach ihrer Ankunft in Schweden (unsystematisch) einem Wohnort zugewiesen wurden, kann dies als natürliches Experiment betrachtet werden. Die Informationen zur Einkommensungleichheit des Wohnortes wurden mit Daten zu Krankenhausaufenthalten verknüpft. In dieser Untersuchung konnte kein $\mathrm{Zu}$ sammenhang von Einkommensungleichheit und Krankenhausaufenthalten gefunden werden.

(Auger et al. 2012) analysieren Daten aus Kanada, um die Effekte von Ungleichheit auf Mortalität bei Migranten und Nicht-Migranten zu untersuchen. Sie kommen zu dem Ergebnis, dass ein Zusammenhang von Einkommensungleichheit und Mortalität bei Nicht-Migranten existiert, jedoch nicht bei Migranten. In Kanada ist die Verteilung der Migranten zwar nicht zufällig, sie siedeln sich eher in Großstädten an, jedoch wird dadurch deutlich, dass die Verteilung des Einkommens innerhalb der unmittelbaren Umgebung zumindest keinen sofortigen Effekt auf die Mortalität hat. Eine ähnliche Herangehensweise wenden (Gadalla/Fuller-Thomson 2008) in der Analyse von US-amerikanischen Bundesstaaten an. Sie kommen zu dem Ergebnis, dass der Zusammenhang von Ungleichheit und zwei untersuchten Indikatoren für körperliche Einschränkungen in einer Teilstichprobe, in der ausschließlich die Personen enthalten sind, die im Staat, in dem sie Leben, auch geboren wurden, stärker ist als in der gesamten Stichprobe, die alle Studienteilnehmer, unabhängig ihres Geburtsortes, enthält. Die Autoren sehen das als Hinweis auf einen kausalen Effekt, da die Personen, die länger (seit der Geburt) in einem Staat leben und damit dessen Ungleichheit ausgesetzt sind - stärker davon beeinflusst werden.

Wie in den vorhergehenden Abschnitten deutlich geworden ist, sind die Ergebnisse der bisherigen Forschung zu den Auswirkungen von Einkommensungleichheit sehr heterogen. Auch wenn es teilweise methodische Erklärungen dafür gibt (siehe Kondo et al. 2012), fallen, wie oben beschrieben, die Interpretationen recht unterschiedlich aus. Die häufig berichteten Korrelationen von Einkommensungleichheit mit verschiedenen Indikatoren von Gesundheit auf Makroebene im Querschnitt sind unbestritten. Eine kausale Interpretation ist in vielen Studien vor allem aufgrund des methodischen Vorgehens (Querschnittsdaten und fehlende Kontrollvariablen) nicht möglich. Analysen, die den Zusammenhang im Längsschnitt untersuchen, sind vorhanden, jedoch ist weitere Forschung nötig, um der Antwort nach der Frage der Kausalität näher zu kommen. Die in dieser Arbeit präsentierten Analysen sind hierfür ein weiterer Schritt. 


\subsubsection{Tests der neomateriellen und psychosozialen Theorie}

Studien haben gezeigt, dass Einkommensungleichheit negativ korreliert ist mit Indikatoren für gesellschaftlichen Zusammenhalt wie Vertrauen (Uslaner/Brown 2005; Kawachi et al. 1997; Elgar et al. 2011; Ichida et al. 2009), Solidarität (Paskov/Dewilde 2012) und Bürgerbeteiligung (Lancee/Van de Werfhorst, Herman G. 2012; Alesina/La Ferrara 2000; Oliver 1999). Die entscheidende Frage ist jedoch, ob - wie in der psychosozialen Hypothese angenommen - geringer sozialer Zusammenhalt einen Einfluss auf die Gesundheit hat. Der aktuelle Forschungsstand hierzu ist uneinheitlich. Während manche Studien einen Zusammenhang von sozialem Vertrauen und Gesundheit feststellen (Subramanian/Kawachi/Kennedy 2001; Snelgrove/Pikhart/Stafford 2009), kann dies in anderen Untersuchungen nur teilweise (Rözer/Volker 2016; Poortinga 2006) oder gar nicht (Elgar et al. 2011; Olsen/Dahl 2007) bestätigt werden.

Unter den zahlreichen Studien, die den Effekt von Einkommensungleichheit auf Gesundheit untersuchen, widmen sich manche auch speziell der Frage, ob dieser Zusammenhang über das soziale Vertrauen verknüpft ist. Mehrere Untersuchungen konnten aufzeigen, dass soziales Vertrauen als Mediator wirkt (Kragten/Rözer 2017; Kawachi et al. 1997; Ichida et al. 2009; Delhey/Dragolov 2014). Weitere Studien können diese Ergebnisse zumindest teilweise bestätigen (Rözer/Volker 2016; Aida et al. 2011).

Es wurden auch zum Teil Interaktionen zwischen der Ungleichheit im Einkommen und dem individuellen sozialen Vertrauen oder Sozialkapital betrachtet. Die Untersuchung von Rözer/Kraaykamp/Huijts (2016) beschreibt, dass das individuelle Maß an sozialem Vertrauen einen Einfluss hat auf den Zusammenhang von Ungleichheit und subjektiver Gesundheit: Ungleichheit ist vor allem schädlich für diejenigen, die viel soziales Vertrauen haben. Bei Personen ohne Vertrauen spielt die Ungleichheit hingegen keine Rolle (ebd.). Van Deurzen et al. (2015) kommen zu dem Ergebnis, dass nichtmaterielle Ressourcen, wie unterstützende soziale Beziehungen, einen Einfluss auf Depression haben, aber der Zusammenhang von Einkommensungleichheit und Depression sich damit nicht erklären lässt.

Die psychosoziale Theorie zur Erklärung des Zusammenhangs von Ungleichheit und Gesundheit bezieht sich neben dem sozialen Kapital und Vertrauen auch auf das Argument, dass soziale Vergleiche bei großer Ungleichheit negative Folgen haben. Empirisch kann gezeigt werden, dass das Ausmaß, in dem Menschen ihr Einkommen vergleichen, nach Ländern variiert. Layte/Whelan (2014) berichten, dass ,status anxiety“, also die Angst von anderen aufgrund der sozialen Position (negativ) bewertet zu werden, in Ländern mit geringer Ungleichheit weniger stark ausgeprägt ist als in Ländern mit größerer Ungleichheit. Mit den Daten des European Social Survey wurde untersucht, wie wichtig den Befragten der Vergleich des Einkommens ist (Clark/Senik 
2010). Die Wichtigkeit des Einkommensvergleichs unterscheidet sich zwischen Ländern, aber auch innerhalb der Länder gibt es Unterschiede: Personen, die in einem Haushalt mit niedrigem Einkommen leben, vergleichen eher ihr Einkommen mit anderen. Insgesamt lässt sich ein moderates Level beobachten, der Einkommensvergleich ist nicht sehr stark ausgeprägt, aber durchaus vorhanden.

Mehrere Studien kommen zu dem Ergebnis, dass auf individueller Ebene eine hohe Vergleichsorientierung mit geringerer Lebenszufriedenheit einhergeht (Goerke/Pannenberg 2015; Mayraz/Wagner/Schupp 2009; Clark/Senik 2010; Delhey/Dragolov 2014). Präg/Mills/Wittek (2014) berichtet zudem einen Zusammenhang mit der subjektiven Gesundheit. Für die Theorie, dass der Pfad von Ungleichheit zu Gesundheit über den sozialen Vergleich oder „status anxiety" vermittelt ist, werden nur in der Studie von Delhey/Dragolov (2014) Hinweise gefunden. In dieser Untersuchung wird allerding nicht Gesundheit, sondern Lebenszufriedenheit betrachtet. Dabei wird berichtet, dass „status anxiety“ ein Mediator des Zusammenhangs in ärmeren Ländern darstellt. Die Arbeiten, die diese Hypothese anhand von Gesundheitsindikatoren überprüft haben, konnten nicht bestätigen, dass sozialer Vergleich ein Mediator der Beziehung von Ungleichheit und Gesundheit darstellt. Anhand derselben Datengrundlage, dem European Social Survey aus den Jahren 2006/2007, ist dies für Depression (van Deurzen/van Ingen/van Oorschot, Wim J. H. 2015) und subjektive Gesundheit (Präg/Mills/Wittek 2014) getestet worden.

Bezüglich der neomateriellen Hypothese kann zunächst festgehalten werden, dass für Europa gezeigt wurde, dass höhere staatliche Gesundheitsausgaben mit besserer Gesundheit (Adeline/Delattre 2017; Bergqvist/Yngwe/Lundberg 2013; siehe aber auch Castilla 2004) und geringeren gesundheitlichen Unterschieden der Bevölkerung (Dahl/van der Wel, Kjetil A 2013) einhergehen. Eine andere Untersuchung zeigt, dass die größten Lücken in der Krankenversicherung in Ländern mit geringen Gesundheitsausgaben und großer Einkommensungleichheit zu finden sind (Jürges 2015). Solche Forschungsergebnisse sprechen für die neomaterielle Theorie, stellen aber keinen expliziten Test dar, wie ihn van Deurzen/van Ingen/van Oorschot, Wim J. H. (2015) und Bhandari/Newton/Bernabé (2015) durchgeführt haben. Beide Studien untermauern die Hypothese, dass der Zusammenhang von Einkommensungleichheit und Gesundheit - zumindest teilweise - durch staatliche Investitionen vermittelt ist.

Neben der bereits genannten Forschung, die die psychosoziale oder die neomaterielle Theorie testen, existieren auch Arbeiten, die beide Theorien anhand gleicher Daten und Methoden gegenüberstellen. In multivariaten Berechnungen, unter Kontrolle von anderen Einflussfaktoren, können Anderson/Bjorklund/Rambotti (2017) keinen signifikanten Zusammenhang von Einkommensungleichheit und drei verschiedenen Gesundheitsindikatoren feststellen. Ihre Ergebnisse sprechen vielmehr für einen Einfluss von staatlichen 
Gesundheitsausgaben und anderen politischen Faktoren. Olsen/Dahl (2007) kommen ebenfalls zu dem Ergebnis, dass unter gegenseitiger Kontrolle weder die Einkommensungleichheit noch soziales Vertrauen (gemessen auf der Länderebene) mit subjektiver Gesundheit verknüpft ist. Dass mit höheren Sozialausgaben des Staates bessere Gesundheit einhergeht, konnte in dieser Analyse nur für Frauen festgestellt werden. Noch detailliertere Analysen der beiden Theorien liefern Elgar (2010) und Layte (2012). Beide Untersuchungen testen, ob soziales Vertrauen bzw. Sozialkapital und staatliche Ausgaben für den Zusammenhang von Einkommensungleichheit und Gesundheit verantwortlich sind. Übereinstimmend berichten die Studien, dass - entgegen den Ergebnissen von Anderson/Bjorklund/Rambotti (2017) sowie Olsen/Dahl (2007) - Vertrauen bzw. Sozialkapital ein Mediator darstellt, staatliche Investitionen hingegen nicht.

\subsubsection{Vermögensungleichheit}

Bislang existieren kaum Studien, die Vermögensungleichheit in Zusammenhang mit der Gesundheit betrachten. Ein Grund hierfür ist, dass es zum Vermögen deutlich weniger Daten gibt als zum Einkommen und die vorhandenen Daten weniger stark vereinheitlicht sind. Nowatzki (2012) untersucht anhand von 14 OECD-Staaten für das Jahr 2000 den Zusammenhang von Vermögensungleichheit mit Lebenserwartung und Säuglingssterblichkeit. Damit ist dies die erste Studie, die die seit langem geforderte Überprüfung der bekannten Ergebnisse von Ungleichheit anhand des Vermögens aufgreift und empirisch testet. Auch unter Kontrolle verschiedener Indikatoren auf staatlicher Ebene u.a. Sozialausgaben, Lohnungleichheit, Großzügigkeit des Rentensystems, Wirtschaftskraft - sind eine geringere Lebenserwartung und eine höhere Säuglingssterblichkeit mit größerer Ungleichheit im Vermögen verbunden. Ein weiteres Ergebnis ist, dass diese Zusammenhänge teilweise über die Großzügigkeit des Rentensystems vermittelt sind. Zudem zeigen die Analysen, dass die Gesundheitsmaße stärker mit Vermögensungleichheit korrelieren als mit der Lohnungleichheit.

Eine andere Situation, was die Verfügbarkeit von Daten bzw. deren Qualität betrifft, ist in Ländern mit mittlerem und geringem Wohlstand vorhanden. Hier werden Ungleichheiten anhand des Vermögens erfasst (vgl. van Deurzen/van Oorschot/van Ingen 2014; La Ferrara 2002; Fox 2012), mit der Begründung, dass dies in armen Gesellschaften ein geeigneteres Maß ist, um die ökonomischen Bedingungen zu erfassen und Informationen zum Einkommen schwieriger zu erheben sind (van Deurzen/van Oorschot/van Ingen 2014: 8). Eine Studie, in der Daten von Frauen aus ärmeren Ländern analysiert wurden, kommt zu dem Ergebnis, dass eine hohe Vermögensungleichheit mit einer grö- 
Beren Wahrscheinlichkeit einhergeht, an Blutarmut zu leiden und den Tod eines Kindes erlebt zu haben (ebd.). Zudem beobachten die Autoren, dass dieser Zusammenhang teilweise über das Gesundheitssystem und die Infrastruktur des Landes vermittelt ist. 


\section{Daten und Methode}

Für die empirische Überprüfung der Forschungsfragen wird auf Daten des Survey of Health, Ageing and Retirement in Europe (SHARE) zurückgegriffen. ${ }^{9}$ SHARE ist eine Panelstudie, in der Personen ab 50 Jahren und ihre Partner u.a. zur finanziellen und gesundheitlichen Situation befragt werden (Börsch-Supan et al. 2013). Für die Analysen werden fünf der sechs verfügbaren Befragungswellen verwendet. Welle 3 kann nicht verwendet werden, denn dabei handelt es sich um eine retrospektive Befragung zum Lebenslauf (SHARELIFE), die sich grundlegend von den restlichen Befragungen unterscheidet. Bei SHARE wird der Fragebogen vor der Befragung harmonisiert, wodurch sichergestellt wird, dass die Daten aus den verschiedenen Ländern bestmöglich vergleichbar sind (Harkness 2005; Börsch-Supan et al. 2013).

Die erste Befragung von SHARE wurde im Jahr 2004 in elf europäischen Ländern und Israel durchgeführt. Im Laufe der Zeit hat sich die Zahl der teilnehmenden Länder erhöht. Die sechste Erhebung fand im Jahr 2015 in 18 Ländern statt. Allerdings sind nicht für alle Länder Daten aus allen Wellen vorhanden. So gibt es bspw. in Portugal, Polen und Griechenland Lücken bei den Befragungen. Zudem weichen die Zeiträume, in denen die Befragungen durchgeführt wurden, teilweise zwischen den Ländern ab. Ein Überblick über die verfügbaren Daten und den Erhebungsjahren von SHARE ist in Tabelle 4.1 dargestellt.

\subsection{Finanzielle Situation}

Die Finanzielle Situation wird in den Analysen anhand von zwei Indikatoren betrachtet: subjektive finanzielle Schwierigkeiten und Verschuldung. Für

9 Diese Arbeit verwendet Daten der SHARE Wellen 1, 2, 4, 5 und 6 in der Version 6.1.1 (DOIs: 10.6103/SHARE.w1.600, 10.6103/SHARE.w2.600, 10.6103/SHARE.w4.600, 10.6103/SHARE.w5.600, 10.6103/SHARE.w6.600; siehe Börsch-Supan et al.(2013) für Informationen zur Methodik). Die Finanzierung der SHARE Datenerhebung erfolgte primär durch die Europäische Kommission, insbesondere durch FP5 (QLK6-CT-2001-00360), FP6 (SHARE-I3: RII-CT-2006-062193, COMPARE: CIT5-CT-2005-028857, SHARELIFE: CIT4-CT-2006-028812) und FP7 (SHARE-PREP: N²11909, SHARE-LEAP: N²27822, SHARE M4: N²61982). Für zusätzliche finanzielle Unterstützung wird dem Bundesministerium für Bildung und Forschung, der Max-Planck-Gesellschaft zur Förderung der Wissenschaften, dem U.S. National Institute on Aging (U01_AG09740-13S2, P01_AG005842, P01_AG08291, P30_AG12815, R21_AG025169, Y1-ĀG-4553-01, IAG_BSR06-11, OGHĀ_04-064, HHSN $271201300071 \bar{C}$ ) sowie zahlreichen nationalen Geldgebern gedankt (siehe www.shareproject.org). 
Tabelle 4.1: Übersicht aller SHARE Wellen und Länder

\begin{tabular}{lcccccc} 
Land & Welle 1 & Welle 2 & $\begin{array}{l}\text { Welle 3 } \\
\text { SHARELIFE }\end{array}$ & Welle 4 & Welle 5 & Welle 6 \\
\hline Österreich & 2004 & $2006 / 07$ & $2008 / 09$ & 2011 & 2013 & 2015 \\
Deutschland & 2004 & $2006 / 07$ & $2008 / 09$ & $2011 / 12$ & 2013 & 2015 \\
Schweden & 2004 & $2006 / 07$ & $2008 / 09$ & 2011 & 2013 & 2015 \\
Niederlande & 2004 & 2007 & $2008 / 09$ & 2011 & 2013 & - \\
Spanien & 2004 & $2006 / 07$ & $2008 / 09$ & 2011 & 2013 & 2015 \\
Italien & 2004 & $2006 / 07$ & $2008 / 09$ & 2011 & 2013 & 2015 \\
Frankreich & $2004 / 05$ & $2006 / 07$ & 2009 & 2011 & 2013 & 2015 \\
Dänemark & 2004 & $2006 / 07$ & $2008 / 09$ & 2011 & 2013 & 2015 \\
Griechenland & $2004 / 05$ & 2007 & $2008 / 09$ & - & - & 2015 \\
Schweiz & 2004 & $2006 / 07$ & $2008 / 09$ & 2011 & 2013 & 2015 \\
Belgien & $2004 / 05$ & $2006 / 07$ & $2008 / 09$ & 2011 & 2013 & 2015 \\
Israel & $2005 / 06$ & $2009 / 10$ & - & - & 2013 & 2015 \\
Tschechien & - & $2006 / 07$ & $2008 / 09$ & 2011 & 2013 & 2015 \\
Polen & - & $2006 / 07$ & $2008 / 09$ & $2011 / 12$ & - & 2015 \\
Irland & - & 2007 & $2009 / 10 / 11$ & - & - & - \\
Luxemburg & - & - & - & - & 2013 & 2015 \\
Ungarn & - & - & - & 2011 & - & - \\
Portugal & - & - & - & 2011 & - & 2015 \\
Slowenien & - & - & - & 2011 & 2013 & 2015 \\
Estland & - & - & - & $2010 / 11$ & 2013 & 2015 \\
Kroatien & - & - & - & - & - & 2015 \\
\hline
\end{tabular}

Quelle: Stuck et al. 2018: 8

die Verwendung dieser Indikatoren sprechen mehrere Punkte. In der Diskussion um ein geeignetes Maß für den sozioökonomischen Status im Alter wird immer wieder darauf verwiesen, dass die üblicherweise verwendeten Indikatoren Bildung, soziale Klasse oder Einkommen für das höhere Alter nicht angemessen und weniger relevant sind (Matthews et al. 2005; Terraneo 2017: 115f; Grundy/Holt 2001). Geeigneter erscheinen hingegen subjektive Einschätzungen der finanziellen Situation und Maße für Deprivation, mit denen gemessen werden kann, ob den Befragten finanzielle Mittel fehlen, um ohne Probleme leben zu können. Diese Maße korrelieren nicht sehr stark mit der Einkommensarmut (Nolan/Whelan 2011; Adena/Myck 2014). Deprivation und subjektive Armut kann in vielen Haushalten festgestellt werden, die nach der Definition der Einkommensarmut nicht als arm gelten. Umgekehrt werden Haushalte als einkommensarm angesehen, die von keinen finanziellen Problemen berichten. Subjektive Armut hängt hingegen stärker vom Vermögen als vom Einkommen ab (Litwin/Sapir 2009). Mit den Daten von SHARE wurde zudem gezeigt, dass subjektiv berichtete finanzielle Probleme mit den objektiven Kriterien materieller Deprivation korrelieren (Adena/Myck/Oczkowska 2015). Ein weiteres Argument, das für die Verwendung der subjektiven finanziellen Situation spricht, ist, dass aus anderen Studien bekannt ist, dass dieses Maß stärker mit der Gesundheit korreliert ist als andere, auf Einkommen basierte Maße (Franzese 2015; Foulds/Wells/Mulder 2014; Rohde et al. 2016; McKenzie et al. 2014). Gegen eine Armutsmessung anhand des Einkommens 
spricht zudem, dass nicht nur das Einkommen sondern auch die nötigen Ausgaben von Bedeutung sind, welche auch durch die Größe und Zusammensetzung des Haushaltes abhängig sind (Terraneo 2017: 115f.). Zudem sind in der Abfrage von genauen Beträgen, sowohl beim Einkommen, als auch beim Vermögen, mit Unwissenheit, Verweigerung und Schätzungen zu rechnen, womit diese Informationen an Genauigkeit verlieren (vgl. Moore/Stinson/Welniak 2000; Schräpler 2016; Meyer/Mok/Sullivan 2009).

\subsubsection{Finanzielle Schwierigkeiten}

Die finanzielle Situation des Haushaltes wird in SHARE u.a. anhand eines Indikators erhoben, der auch als subjektive Armut (vgl. Adena/Myck 2014; Fonseca et al. 2013) oder ,income adequacy“ (Matthews et al. 2005; Litwin/Sapir 2009) bezeichnet wird (siehe Kapitel 2.1). Die Frage lautet: „Wenn Sie jetzt an das gesamte Monatseinkommen Ihres Haushalts denken - was würden Sie dann sagen, wie Ihr Haushalt finanziell über die Runden kommt?". Die Antwortkategorien sind „mit großen Schwierigkeiten“, „mit einigen Schwierigkeiten“, „einigermaßen leicht" und „leicht". Die Frage wird nur einmal pro Haushalt gestellt, auch wenn beide Partner an dem Interview teilnehmen. Dies hat zur Folge, dass über die Wellen hinweg unterschiedliche Personen diese Frage beantworten können. Auch wenn sie zum selben Haushalt gehören, so ist doch eine abweichende Einschätzung der finanziellen Situation möglich. Bei der Verwendung der subjektiven Einschätzung der finanziellen Situation ist jedoch zu bedenken, dass die gegebene Antwort von der mentalen Verfassung der/des Befragten abhängen kann. Dies ist insbesondere im Zusammenhang mit den Analysen zur Depression zu beachten. Es ist möglich, dass ein/e Befragte/r keine Veränderung in der finanziellen Situation erlebt hat, diese jedoch aufgrund einer Depression schlechter einschätzt.

\subsubsection{Schulden}

Neben der subjektiven finanziellen Situation kommt in den Analysen ein weiterer ökonomischer Indikator zum Einsatz, der sowohl das finanzielle als auch das psychische Wohlergehen beeinflussen kann, ein binärer Indikator für Schulden des $/ \mathrm{r}$ Befragten oder des/der Partners/Partnerin (vgl. Hiilamo/Grundy 2018). Hierbei wird jede Art von Schulden berücksichtigt, egal ob es sich um Kredite von Banken, Schulden bei Freunden, überfällige Rechnungen oder Hypotheken handelt. Mit dieser Vorgehensweise wird nicht das Nettovermögen abgebildet, bei dem bspw. der Wert des Hauses mit dem dafür 
aufgenommenen Kredit verrechnet wird. Es geht hier lediglich um die Verpflichtung von zukünftigen Zahlungen, da diese mit psychischen Belastungen verbunden sein können (vgl. ebd.).

\subsection{Gesundheit}

Die Gesundheit wird anhand von drei Indikatoren gemessen, die verschiedene Aspekte abdecken. Mit der Depression steht ein Maß der mentalen Gesundheit im Mittelpunkt der Analysen. Hinzu kommen die Einschränkungen in Aktivitäten des täglichen Lebens, ein Indikator für physische Gesundheit, der v.a. im höheren Alter von Bedeutung ist. Drittens wird die subjektive Gesundheit untersucht. Alle Maße werden zu binären Indikatoren zusammengefasst, mit denen das Vorliegen von Depression, Einschränkungen in Aktivitäten und schlechter Gesundheit beschrieben wird. Auf diese Weise können die Berechnungen für alle Gesundheitsindikatoren auf die gleiche Weise durchgeführt werden. Um sicherzustellen, dass diese Vorgehensweise nicht zu einer Verzerrung der Ergebnisse führt, werden die Analysen von Depression und Einschränkungen in Aktivitäten des täglichen Lebens auch anhand der metrischen Variablen durchgeführt (siehe Kapitel 5.3).

\subsubsection{Depression}

In SHARE werden Symptome einer Depression mit der Euro-D Skala abgefragt. Die Euro-D Skala wurde mit dem Ziel entwickelt ein einheitliches Messinstrument von Depression im Alter zur Verfügung zu stellen, welches in ganz Europa zu validen Ergebnissen führt (Prince et al. 1999). Für den Zeitraum des letzten Monats vor der Befragung werden zwölf Symptome abgefragt, die zu einem Index aufsummiert werden, der somit Werte von 0 bis 12 annehmen kann. Die Symptome sind Traurigkeit, Pessimismus (keine Hoffnungen für die Zukunft), der Wunsch tot zu sein, Schuldgefühle oder Selbstvorwürfe, Schlafstörungen, Desinteresse am Umfeld, Reizbarkeit, Appetitlosigkeit, wenig Energie, Schwierigkeiten mit der Konzentration, keine Freude an Aktivitäten sowie weinen. Aus der Skala wird ein binärer Indikator für Depression gebildet, wobei die empfohlene Einteilung verwendet wird, bei der eine Person ab vier Symptomen als depressiv gilt (ebd.). Ein geringerer Schwellenwert würde vermutlich zu einer hohen Anzahl falscher Depressionsbefunde führen. Denn manche der Symptome können gemeinsam auftreten ohne dass es sich um eine Depression handelt, z.B. kann wenig Energie und Schwierigkeiten mit der Konzentration die Folge von Schlafstörungen sein. 


\subsubsection{Einschränkungen in den Aktivitäten des täglichen Lebens}

Um die physische Gesundheit zu messen, werden die Einschränkungen in Aktivitäten des täglichen Lebens verwendet. Konzeptionell können Aktivitäten des täglichen Lebens (ADL - ,activities of daily living“) und instrumentelle Aktivitäten (IADL - ,instumental activities of daily living“) unterschieden werden. ADL beziehen sich eher auf Körperpflege und Mobilität innerhalb der Wohnung, IADL hingegen auf Aufgaben im Haushalt. Die in SHARE abgefragten ADL sind sich anziehen, durch einen Raum gehen, baden oder duschen, essen, ins Bett legen oder aus dem Bett aufstehen sowie das Benutzen der Toilette. $\mathrm{Zu}$ den IADL zählen das Benutzen einer Karte, um sich in einer fremden Umgebung zurecht zu finden, das Zubereiten einer warmen Mahlzeit, das Einkaufen von Lebensmitteln, telefonieren, Medikamente einnehmen, Haus- oder Gartenarbeit sowie der Umgang mit Geld, zum Beispiel Rechnungen bezahlen oder Ausgaben kontrollieren. ${ }^{10}$ Oftmals werden nur Einschränkungen in ADL untersucht, in dieser Arbeit werden hingegen ADL und IADL zusammengefasst, wie es auch in anderen Studien gemacht wurde (BörschSupan/Bucher-Koenen/Hanemann 2017a; Adena/Myck 2014; Gobbens/van Assen 2014). Theoretisch kann eine Person also bis zu 13 Einschränkungen angeben. Für die Analysen wird allerdings nur zwischen keiner und mindestens einer Einschränkung unterschieden.

\subsubsection{Schlechte subjektive Gesundheit}

Zusätzlich zu Depression und Einschränkungen in den ADL wird auch der subjektive allgemeine Gesundheitszustand untersucht. Dieser wird erhoben anhand der Frage „Würden Sie sagen, Ihr Gesundheitszustand ist..." mit den Antwortmöglichkeiten ,ausgezeichnet“, „,sehr gut“, „gut“, ,mittelmäßig“ und „schlecht“. Diese Antwortkategorien werden so zusammengefasst, dass ein binärer Indikator für schlechte Gesundheit entsteht, wenn ,,mittelmäßig“ oder „schlecht" geantwortet wurde.

10 In Welle 6 wurde zusätzlich noch ,Alleine das Haus verlassen und öffentliche Verkehrsmittel nutzen“ sowie „Wäsche waschen“ in die Liste der IADL aufgenommen. Um Verzerrungen zu vermeiden und die Variable über alle Untersuchungszeitpunkte hinweg konstant zu halten, wurden diese beiden Aktivitäten nicht berücksichtigt. 


\subsection{Makroindikatoren}

Alle Daten auf Individualebene stammen aus SHARE. Aus ihnen wird auch für die deskriptiven Analysen die durchschnittliche Gesundheit der Bevölkerung in den einzelnen Ländern berechnet. Die restlichen Maßzahlen auf Ebene der Nationalstaaten stammen hingegen aus verschiedenen Datenquellen. In den folgenden Abschnitten werden die Daten dargestellt, die in den Analysen verwendet werden. Die zentrale Rolle spielen hierbei die Ungleichheiten im Einkommen und im Vermögen. Für die Überprüfung der psychosozialen und neomateriellen Theorie kommen zudem Indikatoren für soziales Vertrauen und staatliche Investitionen im Gesundheitswesen zum Einsatz.

\subsubsection{Einkommensungleichheit}

Die Verteilung des Einkommens in einer Gesellschaft wird durch die Einkommensungleichheit angegeben. Als Ausgangspunkt hierfür dient die Sortierung der Bevölkerung nach dem Einkommen und ihre Einteilung in gleich große Teilgruppen (Perzentile). Eine gängige Methode ist die Einteilung in zehn Teile (Dezile). Als Beispiel sind in Tabelle 4.2 die Einkommensdezile für Deutschland im Jahr 2016 dargestellt. Hier wird das Vorgehen ersichtlich: Das unterste Dezil bildet die 10 Prozent der Bevölkerung mit dem geringsten Einkommen ab. Sie verfügen über 3,1 Prozent des Einkommens. Das zweite Dezil beschreibt die 10 Prozent der Bevölkerung mit dem zweitgeringsten Einkommensanteil, usw.

Tabelle 4.2: Verteilung des Bruttoäquivalenzeinkommens in Deutschland (2016)

\begin{tabular}{rccc} 
& & \multicolumn{2}{c}{ kummuliert } \\
& \% Einkommen & \% Bevölkerung & $\%$ Einkommen \\
\hline 1. Dezil & 3,1 & 10 & 3,1 \\
2. Dezil & 5,1 & 20 & 8,2 \\
3. Dezil & 6,3 & 30 & 14,5 \\
4. Dezil & 7,3 & 40 & 21,8 \\
5. Dezil & 8,3 & 50 & 30,1 \\
6. Dezil & 9,4 & 60 & 39,5 \\
7. Dezil & 10,6 & 70 & 50,1 \\
8. Dezil & 12,2 & 80 & 62,3 \\
9. Dezil & 14,5 & 90 & 76,8 \\
10. Dezil & 23,3 & 100 & 100,1
\end{tabular}

Quelle: Eurostat (2018b) 
Es existieren verschieden Maßzahlen zur Beschreibung der ungleichen Verteilung des Einkommens (vgl. Latif 2015; Adeline/Delattre 2017). Eine recht einfacher Indikator für die Ungleichverteilung des Einkommens, die in manchen Studien Anwendung findet, ist der Anteil des Einkommens der einkommensstärksten 10 Prozent der Bevölkerung (vgl. Leigh/Jencks 2007; Hill/Jorgenson 2018). Eine andere Variante, die etwas mehr Informationen berücksichtigt, ist die Berechnung des Verhältnisses der Einkommensanteile der Gruppe mit dem höchsten Einkommen im Vergleich zu der Gruppe mit dem geringsten Einkommen (vgl. Pickett/James/Wilkinson 2006; Latif 2015).

Abbildung 4.1: Lorenzkurve des Bruttoäquivalenzeinkommens 2016

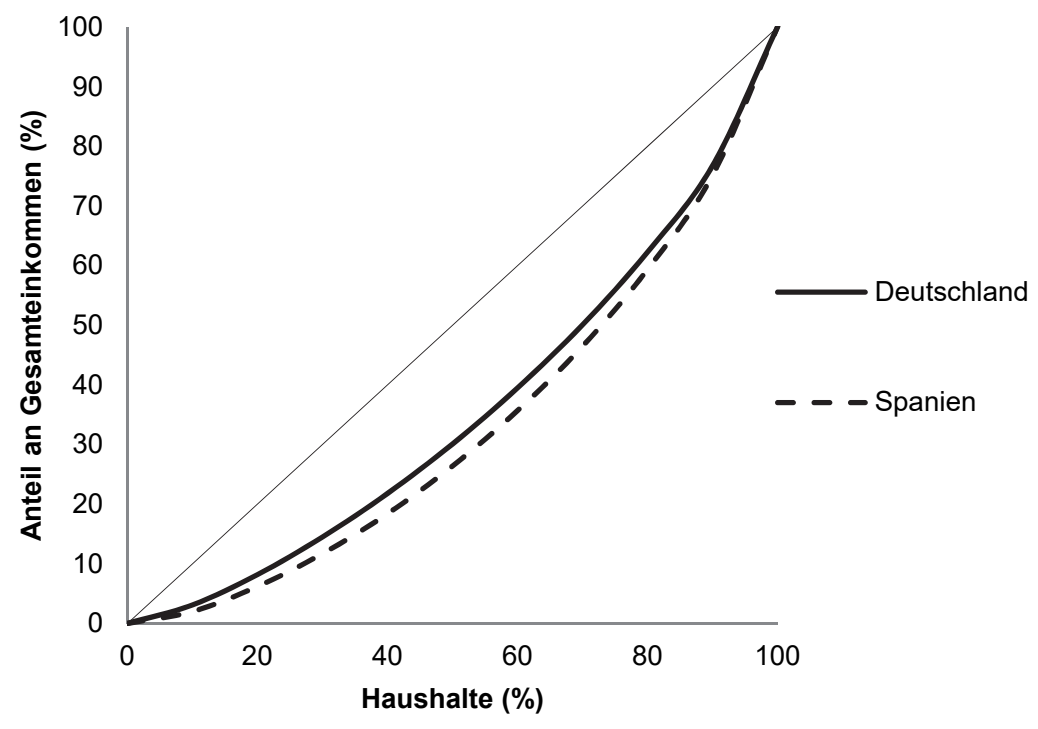

Quelle: Eurostat (2018b). Eigene Darstellung

Die Maßzahl, die jedoch mit Abstand am häufigsten verwendet wird ist der Gini-Koeffizient (Ribeiro et al. 2017). Der Gini-Koeffizient gibt in einer Maßzahl die Verteilung des Einkommens über alle Einkommensgruppen hinweg an. Um die Berechnung des Gini-Koeffizienten zu verstehen, muss zunächst die Lorenzkurve erklärt werden. Hierzu werden, wie in Abbildung 4.1 dargestellt, die kumulierten Werte aus Tabelle 4.2 in ein Koordinatensystem eingetragen. Hierbei gibt die X-Achse den Anteil der Bevölkerung und die Y-Achse den entsprechende Anteil des Einkommens an. Die eingezeichnete 45-GradLinie stellt den Fall einer perfekten Gleichverteilung dar: jeder verfügt über das gleiche Einkommen: 10 Prozent der Bevölkerung haben 10 Prozent des 
Einkommens, 20 Prozent der Bevölkerung haben 20 Prozent des Einkommens, usw. Trägt man die Werte aus Tabelle 4.2 in das Koordinatensystem ein, so erhält man die Lorenzkurve. Sie beschreibt die Verteilung des Einkommens in Deutschland. Je näher diese Kurve an der 45-Grad-Linie ist, desto gleicher ist das Einkommen verteilt. Zum Vergleich ist auch die Einkommensverteilung in Spanien als gestrichelte Linie eingezeichnet. Sie zeigt, dass im Jahr 2016 in Spanien die Ungleichheit größer war als in Deutschland. Der Gini-Koeffizient „gibt den Anteil der realisierten Ungleichheit an der maximal möglichen Ungleichheit an“ (Klein 2016: 260). Um dies zu berechnen, wird die Fläche zwischen der Lorenzkurve und der 45-Grad-Linie (realisierte Ungleichheit) in Verhältnis gesetzt zu der Fläche des Dreiecks unterhalb der 45-Grad-Linie (maximal mögliche Ungleichheit). Für die Berechnung des Gini-Koeffizienten gilt folgende Formel:

$$
G=1-\sum_{i=1}^{n} \frac{\left(L_{i}+L_{i-1}\right)}{2_{n}}
$$

Hierbei bezeichnet $L_{i}$ den Wert der Lorenzkurve am Ende und $L_{i-1}$ den Wert am Anfang des i-ten Perzentils, wobei es $n$ Perzentile mit der Größe 1/n gibt (ebd.: 261). Der Gini-Koeffizient variiert zwischen 0 und 1, wobei ein Wert von 0 eine perfekte Gleichverteilung und ein Wert von 1 die größtmögliche ungleiche Verteilung darstellt. Für die Analysen in Kapitel 7 werden die Werte des Gini-Koeffizienten mit 100 multipliziert um eine bessere Lesbarkeit der Ergebnisse in den multivariaten Analysen zu ermöglichen.

Die Informationen zur Einkommensungleichheit stammen von Eurostat (2016). Die Eurostat-Datenbank stellt für alle EU-Staaten und die Schweiz Daten zur Verfügung, die nach den gleichen Methoden erhoben und berechnet werden. Eurostat beinhaltet keine Informationen von Israel, daher werden die Werte der Einkommensungleichheit in Israel aus der Datenbank der OECD (2016) verwendet. Bei den Berechnungen der OECD gab es im Jahr 2011 eine methodische Umstellung bezüglich der Definition des Einkommens. Daraus resultiert eine geringfügige Abweichung der berechneten Werte der Einkommensungleichheit im Zeitverlauf. Da diese Änderung im Zeitraum liegt, in dem SHARE durchgeführt wurde, werden die Daten von Eurostat (alle Länder außer Israel) mit der Ergänzung der OECD Werte (für Israel) verwendet. Auf diese Weise sind die meisten Informationen durch dieselben Methoden entstanden. Eine Verwendung ausschließlich der Werte der OECD würde hingegen eine Verzerrung im Zeitverlauf aller Länder mitsichführen. 


\subsubsection{Vermögensungleichheit}

Da nur wenige Daten zur Verteilung des Vermögens verfügbar sind, ist es nicht möglich, die Ungleichheit im Vermögen im Zeitverlauf zu betrachten. Das Credit Suisse Research Institute (2016) liefert eine umfangreiche Sammlung an Daten zum Vermögen und Vermögensungleichheit. Hier ist für jedes Land dieser Untersuchung die Ungleichheit anhand eines Gini-Koeffizienten berechnet worden. Die Informationen sollten jedoch mit Vorsicht behandelt werden, da sie auf unterschiedlichen Datenquellen und Methoden beruhen. So ist die Untersuchungseinheit in den meisten Fällen der Haushalt oder die Familie, manchmal jedoch auch Individuen. Zudem werden meist Umfragedaten für die Berechnungen verwendet, in Schweden und der Schweiz basieren die Informationen hingegen auf Registerdaten. Die Daten stammen außerdem aus verschiedenen Jahren, zum Großteil aus 2009 oder 2010, jeweils ein Land aus 2008 und 2007, nur die Schweiz weicht mit Informationen aus 1997 stärker ab. Die größte Einschränkung der Daten ist, dass für Estland, Israel, Polen und Tschechien keine Informationen zur Vermögensungleichheit vorliegen und diese anhand von anderen Daten - hauptsächlich Einkommen - imputiert wurden. In den Analysen in Kapitel 7 wird auch die Vermögensungleichheit anhand des Gini-Koeffizienten angegeben und mit 100 multipliziert.

\subsubsection{Soziales Vertrauen}

Ob der Zusammenhang von Ungleichheit und Gesundheit über soziales Vertrauen vermittelt ist, wie es die psychosoziale Theorie beschreibt, ist eine der Forschungsfragen dieser Arbeit. Um dies zu testen werden Informationen zum sozialen Vertrauen in den einzelnen Ländern verwendet, die aus dem European Social Survey (ESS) stammen. Der ESS ist eine Querschnittsstudie, die seit 2002 alle zwei Jahre in vielen Europäischen Ländern durchgeführt wird (European Social Survey 2016). Erfragt werden Einstellungen und Meinungen zu einer Vielzahl von Themen, so auch zu sozialem Zusammenhalt und Vertrauen zu den Mitmenschen. Anhand von drei Fragen, die in jeder Welle des ESS enthalten sind, kann ein Index erstellt werden, die „Social Trust Scale“ (Breyer 2015). Abgefragt wird erstens, inwieweit man anderen Menschen vertraut, zweitens, ob man glaubt, dass sich die meisten anderen Menschen fair verhalten, und drittens, ob andere Menschen hilfsbereit sind. Alle Fragen werden mit einem Wert auf einer Skala von 0 bis 10 beantwortet. Der Index für soziales Vertrauen wird gebildet, indem die angegebenen Werte von allen drei Fragen addiert werden. Je höher der Wert, desto größer ist das soziale Vertrauen des Befragten. Durch die regelmäßigen Erhebungen des ESS können die Daten der einzelnen Wellen den SHARE Wellen zugeordnet werden, dies ist in Tabelle 
4.3 dargestellt. Für die Analysen werden somit die ESS-Wellen 2 bis 7 verwendet (ESS 2004, 2006, 2008, 2010, 2012, 2014).

Tabelle 4.3: Zuordnung von ESS und SHARE Wellen

\begin{tabular}{ll} 
SHARE & ESS \\
\hline Welle $1(2004 / 2005)$ & Welle $2(2004 / 2005)$ \\
Welle 2, außer Israel (2006/2007) & Welle $3(2006 / 2007)$ \\
Welle 2, Israel (2009) & Welle $4(2008 / 2009)$ \\
Welle 4 (2010/2011) & Welle $5(2010 / 2011)$ \\
Welle 5 (2013) & Welle 6 (2012/2013) \\
Welle 6 (2015) & Welle $7(2014 / 2015)$ \\
\hline
\end{tabular}

Quelle: SHARE, ESS. Eigene Darstellung

\subsubsection{Gesundheitsausgaben}

Um die neomaterielle Theorie zu testen, wird ein Maß für staatliche Ausgaben in der Gesundheitsversorgung der Bevölkerung verwendet. Anstatt das Verhältnis von staatlichen Gesundheitsausgaben und dem Bruttoinlandsprodukt zu betrachten (vgl. Olsen/Dahl 2007; Elgar 2010; Layte 2012), wird hier auf denAnteil der von der Bevölkerung privat bezahlten Ausgaben an den gesamten Gesundheitsausgaben zurückgegriffen (vgl. Klein/Unger 2001). Die Daten stammen aus der OECD-Datenbank und sind, abgesehen von Griechenland, für alle Länder über den gesamten Beobachtungszeitraum verfügbar (OECD 2018a). Mit diesem Indikator wird abgebildet, wie stark individuelle ökonomische Ressourcen mit der Möglichkeit einer medizinischen Versorgung verknüpft sind. Zudem hängt dieses Maß nicht von der wirtschaftlichen Lage des Landes ab, wie es im Fall der Gesundheitsausgaben in Relation zum Bruttoinlandsprodukt der Fall ist.

\subsection{Kontrollvariablen}

Neben der finanziellen Situation des Haushaltes und den beschriebenen Makroindikatoren wird in den multivariaten Analysen noch auf weitere Faktoren, die die Gesundheit beeinflussen, kontrolliert. Hierbei kann zwischen zeitkonstanten und zeitveränderlichen Faktoren unterschieden werden. Zeitkonstante Eigenschaften sind nur für manche der angewendeten Methoden relevant 
(siehe Kapitel 4.6). Das Geschlecht wird kontrolliert, um die Unterschiede in der Gesundheit, aber auch im Antwortverhalten - v.a. bei mentaler Gesundheit - zu erfassen. Mit dem Land werden kulturelle Unterschiede im Antwortverhalten und im Gesundheitssystem sowie andere gesellschaftliche Bedingungen berücksichtigt. Die Bildung ist von Bedeutung, da sie eng mit beruflichen Chancen, Einkommen und Arbeitsbedingungen verknüpft ist (vgl. Kapitel 2.2.3). Operationalisiert ist der Bildungsabschluss in den Kategorien „keine oder Grundschulbildung“, „,höhere Schulbildung“ und „Hochschulabschluss“.

Da sich das Land, das Geschlecht und die Bildung in der Stichprobe im Zeitverlauf nicht ändern ${ }^{11}$, werden diese Variablen in den Fixed-Effects-Berechnungen nicht berücksichtigt (siehe Kapitel 4.6.3). Es gibt jedoch eine Reihe von Faktoren, die zeitveränderlich sind und daher in die Modelle aufgenommen werden sollten. Dazu gehört zweifelsohne das Alter, denn vor allem in der zweiten Lebenshälfte wird die Gesundheit mit zunehmendem Alter immer schlechter (Leopold/Engelhardt/Engelhartdt 2013; Jylhä et al. 2001). Die Gesundheit steht auch mit dem Partnerschaftsstatus in Zusammenhang. Viele Studien belegen, dass Ehen und Partnerschaften mit besserer mentaler und physischer Gesundheit verknüpft sind (Schütz 2019; Rapp/Stauder 2019; Grundy/Tomassini 2010; Lorant et al. 2007). Als Mechanismen hinter diesem Zusammenhang werden soziale Kontrolle und Unterstützung, aber auch ökonomische Skaleneffekte genannt. In der Längsschnittanalyse - in der die Veränderung von Zuständen untersucht wird - ist vor allem der Verlust des Partners durch Trennung oder Tod von Bedeutung, da dies insbesondere für die mentale Gesundheit eine Belastung darstellt (Lindeboom/Portrait/van den Berg, Gerard J 2002). Zudem kann eine Verwitwung der Grund für eine Verschlechterung der finanziellen Lage sein. In den Analysen wird daher berücksichtigt, ob der oder die Befragte zusammen mit einem Partner im Haushalt lebt. Ein weiterer Faktor, der sowohl mit der finanziellen Situation, als auch mit der Gesundheit in Verbindung steht, ist der Erwerbsstatus. Z.B. kann schlechte Gesundheit der Grund für einen vorgezogenen Renteneintritt sein (Rice et al. 2011; Börsch-Supan/Bucher-Koenen/Hanemann 2017b) und Arbeitslosigkeit stellt eine psychische Belastung dar (Weich/Lewis/Jenkins 2001). Eine Studie mit Daten von SHARE kommt zu dem Ergebnis, dass der Eintritt in die Rente einen positiven Effekt auf die Gesundheit hat (Hanemann 2018). In den multivariaten Analysen wird daher zwischen Personen in Ruhestand, Erwerbstätigen, Arbeitslosen, Erwerbsunfähigen, Hausfrauen und männern sowie einer Kategorie für sonstige Gruppen unterschieden. Neben den genannten ökonomischen Einflussfaktoren soll so gut wie möglich die fi-

11 In SHARE wird ein/e Befragte/r nicht weiterverfolgt sobald er/sie in ein anderes Land umzieht. Der Bildungsabschluss kann sich auch im höheren Alter noch ändern, allerdings dürfte das sehr selten der Fall sein. In SHARE wird die Bildung daher nur einmal, bei der ersten Teilnahme, erhoben. 
nanzielle Situation des Haushaltes dargestellt werden, daher wird in den multivariaten Analysen auch darauf kontrolliert, ob der Haushalt Miete zahlt oder nicht. Die Kategorie „mietfrei“ besteht hierbei zum Großteil aus Haus- bzw. Wohnungsbesitzern, zu einem kleinen Teil aber auch aus Haushalten, die in einer Wohnung, die nicht ihnen gehört, mietfrei wohnen. Zudem sind in den Berechnungen auch die Informationen enthalten, ob die Befragten oder ihre Partner in dem Jahr vor der Befragung ein größeres Geschenk oder eine Erbschaft erhalten haben. Mit Variablen für die Untersuchungswelle werden Periodeneffekte abgebildet. So kann z.B. die Finanzkrise, die im Untersuchungszeitraum ausgebrochen ist, berücksichtigt werden. Zudem enthalten die Berechnungen eine Variable, die angibt, ob die befragte Person selbst oder ihr Partner die Frage zur finanziellen Situation des Haushaltes beantwortet hat. Dies ist von Bedeutung, da es sich um eine subjektive Einschätzung handelt und die Person, die die Fragen zum Haushalt beantwortet, zwischen den Wellen wechseln kann.

$\mathrm{Ob}$ es angebracht ist, in den multivariaten Berechnungen, bei denen Gesundheit die abhängige Variable ist, auf andere Gesundheitsindikatoren zu kontrollieren, hängt von der jeweiligen Fragestellung ab. Misst die abhängige Variable eine ähnliche Dimension von Gesundheit wie die potentielle Kontrollvariable, so sollte diese nicht in das Modell mitaufgenommen werden. Das Ergebnis wäre, dass Gesundheit durch Gesundheit erklärt werden würde. Wird mit der Kontrollvariable jedoch ein anderer Aspekt der Gesundheit gemessen als mit der abhängigen Variable, dann kann die Berücksichtigung gerechtfertigt sein. Zudem gilt es zu beachten, welchen Zusammenhang man zwischen unabhängiger, abhängiger und Kontrollvariable vermutet und welchen Zusammenhang man messen will. Wie in Abbildung 4.2 dargestellt, wird angenommen, dass die finanzielle Situation die mentale Gesundheit beeinflusst. ${ }^{12}$ Erstens durch einen direkten Effekt, z.B. aufgrund von Zukunftsängsten und psychischem Stress durch Schulden. Zweitens besteht ein indirekter Effekt, der über physische Gesundheit vermittelt wird, d.h. durch finanzielle Probleme entstehen physische Krankheiten, die wiederum die mentale Gesundheit negativ beeinflussen. Dasselbe Schema kann auch angenommen werden, wenn mentale und physische Gesundheit in ihren Rollen als Mediator und abhängige Variable vertauscht werden. Will man wissen wie stark der Zusammenhang von finanzieller Situation und mentaler Gesundheit insgesamt ist, d.h. unabhängig vom Mechanismus, dann darf die physische Gesundheit nicht kontrolliert werden. Ist man jedoch am direkten Effekt von finanzieller Situation auf die mentale Gesundheit interessiert, dann muss die physische Gesundheit berücksichtigt werden.

12 Die Abbildung stellt lediglich ein vereinfachtes Schema dar. Zur besseren Übersicht wurde auf Pfeile für umgekehrte Kausalität verzichtet. 
Abbildung 4.2: Schema für den direkten und indirekten Effekt von finanzieller Situation auf die mentale Gesundheit

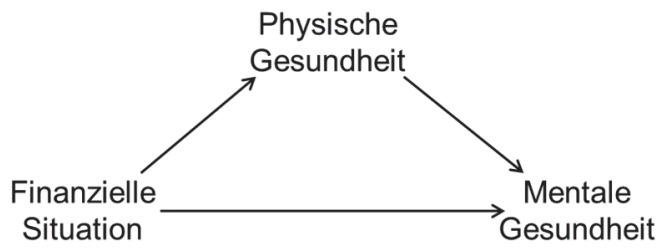

Quelle: Eigene Darstellung

In den hier vorgestellten Analysen werden bei Betrachtung der mentalen Gesundheit (Depression) die beiden Indikatoren der physischen Gesundheit kontrolliert. Umgekehrt wird bei den Analysen der physischen Gesundheit (Einschränkungen in ADL und subjektive Gesundheit) nur die mentale Gesundheit kontrolliert.

In den Analysen zu Einkommens- und Vermögensungleichheit (Kapitel 7) wird der Wohlstand des Landes, gemessen anhand des Bruttoinlandsprodukts (BIP), als Kontrollvariable mitaufgenommen. Das BIP ist eine wichtige Kontrollvariable, da in anderen Studien gezeigt wurde, dass der Zusammenhang von Ungleichheit und Gesundheit unter Kontrolle des BIP geringer wird (Lynch et al. 2000; van Deurzen/van Ingen/van Oorschot, Wim J. H. 2015). Das BIP eines Landes korreliert mit dessen Gesundheitsausgaben (van Deurzen/van Oorschot/van Ingen 2014) und zumindest bis zu einem gewissen Grad verbessert sich die Gesundheit der Bevölkerung bei steigendem Wohlstand (vgl. Preston 2007). Dass auch kurzfristige Veränderungen in der Wirtschaftskraft einer Gesellschaft Auswirkungen auf die Gesundheit haben können, zeigt eine Studie der letzten großen Finanzkrise (Karanikolos et al. 2013). Die Daten zum BIP stammen von der OECD (2018b), wobei die Werte des BIP pro Kopf, zu konstanten Preisen und kaufkraftbereinigt (an US-Dollar 2010) verwendet werden. Es wird angenommen, dass der positive Effekt von höherem Wohlstand auf die Gesundheit mit höherem Wohlstandsniveau abnimmt, so wie es Preston bereits 1975 für Mortalität beobachtet und beschrieben hatte (Preston 2007). In den Analysen wird daher der Wert des BIP logarithmiert, um den bekannten Zusammenhang von Wohlstand einer Gesellschaft und Gesundheit abzubilden (vgl. Kragten/Rözer 2017). 


\subsection{Stichprobe}

Die Informationen aller Befragten der SHARE-Erhebungswellen 1, 2, 4, 5 und 6 werden zu einem Längsschnittdatensatz zusammengefügt. In diesen Wellen sind Daten von insgesamt 120.047 Personen enthalten. Für die Analysen werden jedoch nicht alle vorhandenen Informationen verwendet. Die Definition der Stichprobe erfordert den Ausschluss von Fällen, dies ist in Abbildung 4.3 dargestellt. Da für die Längsschnittanalysen mindestens zwei Beobachtungszeitpunkte pro Person notwendig sind, können die Daten aus Irland, Ungarn und Kroatien nicht analysiert werden. In diesen Ländern wurde die reguläre SHARE-Erhebung jeweils nur einmal durchgeführt. Alle Beobachtungen, bei denen die Befragungsperson jünger als 50 Jahre war, werden gelöscht. Zwar werden bei der Stichprobenziehung in SHARE nur Personen ab 50 Jahren ausgewählt, allerdings werden auch Partner interviewt, wenn diese im selben

Abbildung 4.3: Generierungsprozess der Analysestichprobe

\begin{tabular}{|c|c|c|c|}
\hline $\begin{array}{c}\text { Welle 1 } \\
30.434 \\
\text { Personen }\end{array}$ & $\begin{array}{c}\text { Welle 2 } \\
37.174 \\
\text { Personen }\end{array}$ & $\begin{array}{c}\text { Welle 4 } \\
58.184 \\
\text { Personen }\end{array}$ & $\begin{array}{c}\text { Welle } 5 \\
66.221 \\
\text { Personen }\end{array}$ \\
Personen
\end{tabular}

Quelle: SHARE Rel. 6.1.1, Wellen 1, 2, 4, 5, 6. Eigene Berechnungen. Eigene Darstellung. 
Haushalt leben, unabhängig von ihrem Alter. Die Daten der Partner ab 50 Jahren werden für die Analyse verwendet. Fälle, die fehlende Werte in mindestens einer der Variablen haben, die in den Analysen verwendet werden, werden ebenfalls gelöscht. Im letzten Schritt kommen nur die Personen in die Analysestichprobe, die mindestens zwei Beobachtungszeitpunkte ohne fehlende Werte aufweisen. Nach Ausschluss von Beobachtungen nach diesen Kriterien stehen 189.647 Beobachtungen von 67.599 Personen zur Verfügung mit denen die Analysen durchgeführt werden.

Über alle Wellen fallen knapp 8 Prozent der verfügbaren Beobachtungen aus den Analysen aufgrund fehlender Werte in einer der benötigten Variablen. Eine Untersuchung der Fälle mit fehlenden Informationen zeigt, dass fehlende Werte eher bei Personen vorkommen, die schlechte Gesundheit haben, über einen geringen Bildungsabschluss verfügen und in einem Haushalt mit finanziellen Problemen leben. Somit ist die Analysestichprobe bezüglich dieser Eigenschaften leicht verzerrt.

In der Stichprobe befinden sich etwas mehr Frauen als Männer. Das Durchschnittsalter über alle Beobachtungen liegt bei ca. 66 Jahren. Bei etwa 10 Prozent der Fälle wird im Haushalt von großen finanziellen Schwierigkeiten berichtet. In über 64 Prozent der Beobachtungen kommen die Befragten einfach oder einigermaßen einfach über die Runden. Von Schulden wird in ca. 29 Prozent der Fälle berichtet. Was die Gesundheitsindikatoren betrifft, so ist die subjektive Gesundheit das Maß, bei dem am häufigsten schlechte Gesundheit berichtet wird, in über 36 Prozent der Fälle. Depression - nach der Definition von 4 oder mehr Symptomen - tritt bei 26 Prozent und Einschränkungen in ADL bei 19 Prozent der Beobachtungen auf. Bei deutlich über der Hälfte der Beobachtungen gibt der oder die Befragte an in Ruhestand zu sein. Der Anteil der Rentner dürfte jedoch noch etwas höher sein, da vor allem Frauen oftmals im hohen Alter trotz Rentenbezug als Erwerbsstatus „Hausfrau/-mann“ angeben. Tabelle 4.4 bietet einen Überblick über die Stichprobe.

Durchschnittlich sind pro Person 2,8 Beobachtungen vorhanden. 46,7 Prozent der Personen sind mit nur zwei Beobachtungen in der Stichprobe vertreten. Drei Beobachtungszeitpunkte gibt es von 34,1 Prozent der Personen. Vier Beobachtungen liegen nur für 11 Prozent und fünf Beobachtungen nur für 8,2 Prozent der Personen vor. Zudem stammen mehr Beobachtungen aus den späteren SHARE-Wellen, vor allem aus Welle 5 und 6. Dies lässt sich durch größere Stichproben und mehr teilnehmenden Ländern erklären. Eine Übersicht über die Fallzahlen in der Analysestichprobe nach Land und Welle ist in Tabelle 4.5 dargestellt.

Eine Besonderheit von SHARE ist, dass die Befragten auch in Alten- und Pflegeheimen interviewt werden. In der hier verwendeten Stichprobe sind allerdings nur Personen in Privathaushalten enthalten, da bei Interviews in Pflegeheimen ein verkürzter Fragebogen zum Einsatz kommt, der die Frage nach der finanziellen Situation nicht enthält. 
Tabelle 4.4: Beschreibung der Stichprobe

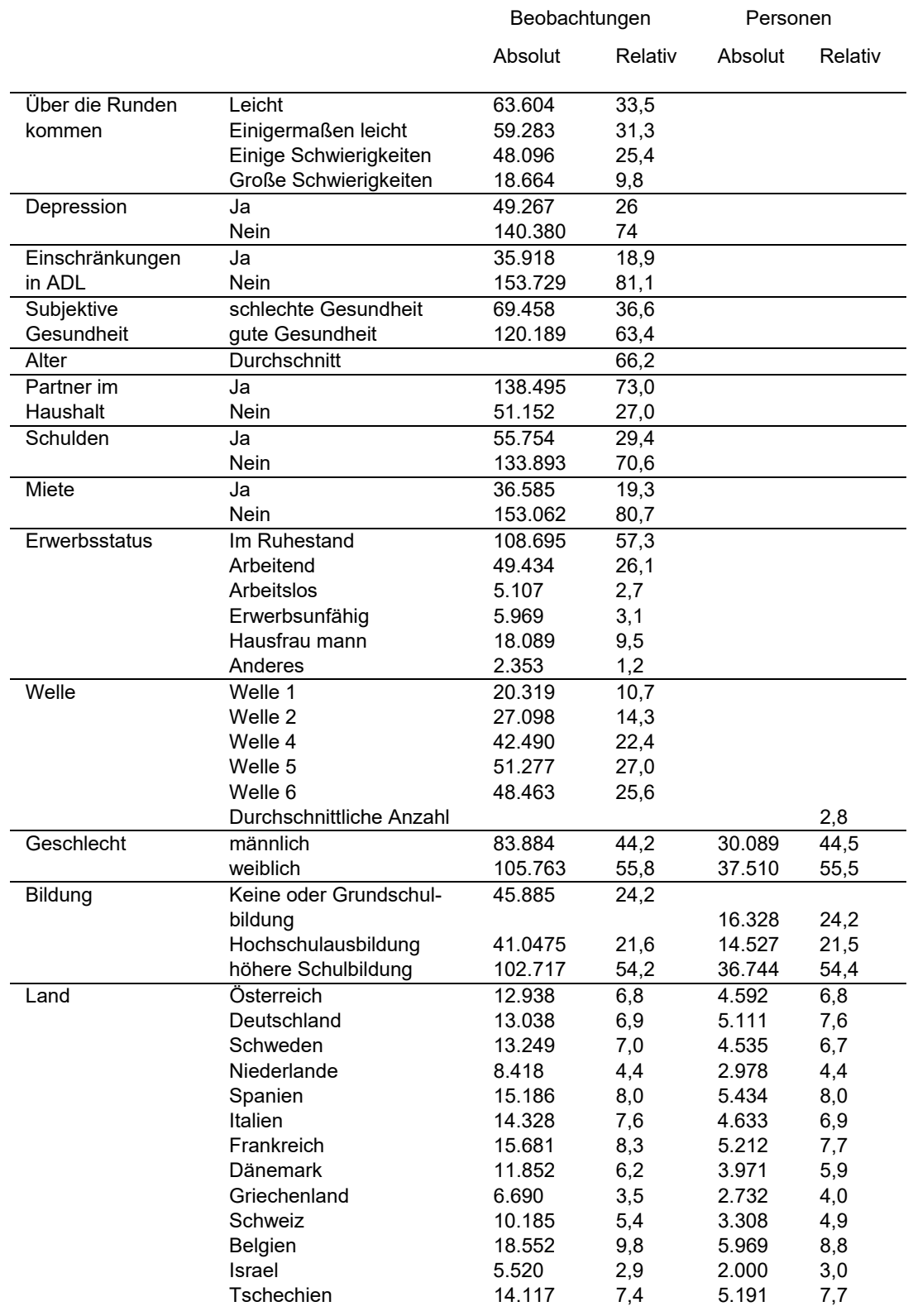


Fortsetzung der Tabelle 4.4: Beschreibung der Stichprobe

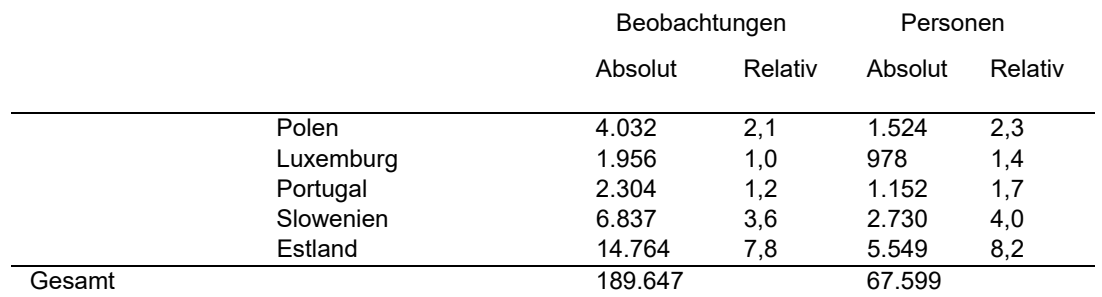

Quelle: SHARE Rel. 6.1.1, Wellen 1, 2, 4, 5, 6. Eigene Berechnung.

Eine große Anzahl an Befragten wird aus den Analysen ausgeschlossen, weil sie nur einmal an der Befragung teilgenommen haben. Daher ist es angebracht die Analysestichprobe mit den ausgeschlossenen Fällen zu vergleichen, um mögliche Verzerrungen der Stichprobe erkennen zu können. Die Fälle, die in den Analysen nicht berücksichtigt werden, da sie nur eine Beobachtung aufweisen, sind in den meisten Variablen der Analysestichprobe sehr ähnlich (siehe Onlineanhang 1). Die ausgeschlossenen Fälle haben bei allen Gesundheitsindikatoren einen etwas höheren Anteil an Personen mit schlechter Gesundheit. Dass kranke und depressive Personen eine höhere Ausfallquote in Panelbefragungen haben, wurde auch in anderen Panelstudien beobachtet (Mirowsky/Reynolds 2000; Chatfield/Brayne/Matthews 2005; zu einem anderen Ergebnis gelangen jedoch Banks/Muriel/Smith 2011). Zudem ist der Anteil

Tabelle 4.5: Fallzahlen pro Land und Welle

\begin{tabular}{lcccccr} 
& Welle 1 & Welle 2 & Welle 4 & Welle 5 & Welle 6 & Gesamt \\
\hline Österreich & 1.151 & 1.113 & 3.912 & 3.825 & 2.937 & 12.938 \\
Deutschland & 1.590 & 1.934 & 1.466 & 4.090 & 3.958 & 13.038 \\
Schweden & 2.203 & 2.345 & 1.780 & 3.499 & 3.422 & 13.249 \\
Niederlande & 1.858 & 2.099 & 2.312 & 2.149 & - & 8.418 \\
Spanien & 1.536 & 1.864 & 2.913 & 4.619 & 4.254 & 15.186 \\
Italien & 1.825 & 2.460 & 2.841 & 3.713 & 3.489 & 14.328 \\
Frankreich & 2.028 & 2.294 & 4.220 & 3.980 & 3.159 & 15.681 \\
Dänemark & 1.201 & 2.044 & 2.024 & 3.413 & 3.170 & 11.852 \\
Griechenland & 2.178 & 2.619 & - & - & 1.893 & 6.690 \\
Schweiz & 673 & 1.194 & 2.985 & 2.796 & 2.537 & 10.185 \\
Belgien & 2.729 & 2.835 & 4.150 & 4.669 & 4.169 & 18.552 \\
Israel & 1.347 & 1.560 & - & 1.389 & 1.224 & 5.520 \\
Tschechien & - & 1.272 & 4.054 & 4.584 & 4.207 & 14.117 \\
Polen & - & 1.465 & 1.425 & - & 1.142 & 4.032 \\
Luxemburg & - & - & - & 978 & 978 & 1.956 \\
Portugal & - & - & 1.152 & - & 1.152 & 2.304 \\
Slowenien & - & - & 1.975 & 2.468 & 2.394 & 6.837 \\
Estland & - & - & 5.281 & 5.105 & 4.378 & 14.764 \\
\hline Gesamt & 20.319 & 27.098 & 42.490 & 51.277 & 48.463 & 189.647
\end{tabular}

Quelle: SHARE Rel. 6.1.1, Wellen 1, 2, 4, 5, 6. Eigene Berechnungen. 
an Personen mit finanziellen Problemen im Haushalt bei der Befragten mit nur einer Beobachtung höher als in der Analysestichprobe.

Für bestimmte Fragestellungen und Robustheitsanalysen werden weitere (Unter-) Stichproben verwendet, die in der Verteilung der Variablen ebenfalls zum größten Teil nur geringfügig von dem Analysesample abweichen. An den entsprechenden Stellen in den Analysekapiteln wird auf die abweichende Stichprobe verwiesen.

\subsubsection{Veränderungen im Zeitverlauf}

Für eine Längsschnittanalyse ist es essentiell, dass in den zeitveränderlichen Variablen ausreichend zeitliche Variation besteht. Daher soll nun betrachtet werden, wie häufig Veränderungen in der finanziellen Situation im Analysesample vorkommen. Dazu wird in Tabelle 4.6 in einer Kreuztabelle die finanzielle Situation der Haushalte zu zwei aufeinanderfolgenden Zeitpunkten anhand von bedingten Häufigkeiten dargestellt. Die Tabelle zeigt zeilenweise Prozentangaben der finanziellen Situation zum Zeitpunkt $t_{1}$, abhängig von der finanziellen Situation zu Zeitpunkt $t_{0}$. Am stabilsten ist die Lage von Haushalten, die leicht über die Runden kommen, ca. 59 Prozent sind auch bei der darauffolgenden Beobachtung in dieser Kategorie. Ein Abstieg zu großen Schwierigkeiten ist hingegen selten, nur 1,6\% der Haushalte ohne finanzielle Probleme rutschen in die unterste Kategorie. Aus der Tabelle wird jedoch auch deutlich, dass es durchaus Veränderungen im Zeitverlauf gibt, was die finanzielle Lage der Haushalte betrifft. Vor allem Auf- und Abstiege in die benachbarten Kategorien kommen häufig vor, wobei Verbesserungen häufiger sind als Verschlechterungen. Während ca. 30 Prozent der Haushalte, die einige finanzielle Schwierigkeiten hatten, aufsteigen zu ,einigermaßen leicht", steigen nur 16 Prozent dieser Haushalte in die unterste Kategorie ab. Und immerhin 43,7 Prozent der Haushalt mit großen Schwierigkeiten berichten in der nächsten Befragung nur einige Schwierigkeiten. Diese Muster bestehen in den meisten Ländern, dennoch gibt es auch hier Ausnahmen. Portugal und Griechenland stechen hervor, da hier mehr Ab-als Aufstiege zu beobachten sind (siehe Tabellen nach Länder in Onlineanhang 2).

Für die Längsschnittanalysen sollte auch Variation in den abhängigen Variablen vorhanden sein. In gleicher Weise wie in Tabelle 4.6 zeigt Tabelle 4.7 die Veränderung in den Gesundheitsindikatoren. Hieraus wird ersichtlich, dass es häufig vorkommt, dass eine Person von schlechtem zu gutem Gesundheitszustand wechselt. Ca. 44 Prozent der depressiven gelten in der darauffolgenden Befragung nicht mehr als depressiv. Die Wahrscheinlichkeit depressiv zu werden liegt in der Stichprobe bei ca. 16 Prozent. Oben wurde bereits erwähnt, dass es unwahrscheinlicher ist, dass Kranke und Depressive bei Wiederho- 
Tabelle 4.6: Veränderung in der finanziellen Situation der Haushalte

\begin{tabular}{l|lllll} 
t0 & $\begin{array}{l}\mathrm{t} 1 \\
\text { Große } \\
\text { Schwierigkeiten }\end{array}$ & $\begin{array}{l}\text { Einige } \\
\text { Schwierigkeiten }\end{array}$ & $\begin{array}{lllll}\text { Einigermaßen } \\
\text { leicht }\end{array}$ & Leicht & Gesamt \\
\hline große Schwierigkeiten & 38,1 & 43,7 & 12,9 & 5,3 & 100,0 \\
einige Schwierigkeiten & 16,0 & 40,4 & 30,6 & 13,0 & 100,0 \\
einigermaßen leicht & 3,7 & 22,3 & 38,4 & 35,6 & 100,0 \\
leicht & 1,6 & 8,2 & 31,3 & 58,9 & 100,0 \\
\hline
\end{tabular}

Quelle: SHARE Rel. 6.1.1, Wellen 1, 2, 4, 5, 6. Eigene Berechnungen

lungsbefragung mitmachen, daher sind diese Zahlen nicht als „wahre Werte“ zu verstehen. Sie verdeutlichen jedoch, dass die Stichprobe ausreichend zeitliche Variation bezüglich der Depression enthält. Gleiches gilt für die Einschränkungen in den ADL sowie für die subjektive Gesundheit. Am unwahrscheinlichsten ist eine Verschlechterung der Gesundheit gemessen anhand der körperlichen Einschränkungen. Subjektive Gesundheit ist der Indikator, bei dem am seltensten eine Verbesserung beobachtet wird.

Tabelle 4.7: Veränderung in der Gesundheit

\begin{tabular}{l|lll} 
to & $\mathbf{t 1}$ & \multicolumn{2}{l}{ Zeilenprozent } \\
& & & \\
& keine Depression & Depression & Gesamt \\
\hline $\begin{array}{l}\text { keine Depression } \\
\text { Depression }\end{array}$ & 84,1 & 15,9 & 100,0 \\
& 44,4 & 55,6 & 100,0 \\
& keine ADL & ADL & Gesamt \\
\hline keine ADL & 87,8 & 12,2 & 100,0 \\
ADL & 41,4 & 58,6 & 100,0 \\
& & & \\
& gute subjektive & schlechte subjektive & \\
\hline gute subjektive Gesundheit & 80,6 & Gesundheit & Gesamt \\
schlechte subjektive Gesundheit & 26,8 & 19,4 & 100,0 \\
& & 73,2 & 100,0
\end{tabular}

Quelle: SHARE Rel. 6.1.1, Wellen 1, 2, 4, 5, 6. Eigene Berechnungen.

\subsection{Analysemethoden}

Für die Analyse der Forschungsfragen kommen verschiedene Methoden zum Einsatz, die jedoch eine Gemeinsamkeit haben: alle sind lineare Wahrscheinlichkeitsmodelle. Das bedeutet, dass ein lineares Modell geschätzt wird, wobei 
die abhängige Variable binär kodiert ist. Abbildung 4.4 zeigt ein fiktives Beispiel für einen Zusammenhang von Einkommen und Depression $(0=$ keine Depression; 1=Depression). Ein höheres Einkommen ist hier mit einem geringeren Depressionsrisiko verknüpft. Da binär kodierte abhängige Variablen oftmals mit einem Logit oder Probit-Modell analysiert werden, soll kurz erläutert werden, warum in dieser Arbeit auf ein lineares Wahrscheinlichkeitsmodell zurückgegriffen wird. Daher sind in Abbildung 4.4 alle Beobachtungen sowie die Vorhersagewerte von einem linearen Wahrscheinlichkeitsmodell und einem Logit-Modell dargestellt.

Das Logit-Modell ist, wie auch das Probit-Modell, speziell für binäre abhängige Variablen entwickelt worden (Best/Wolf 2010; Andreß/Golsch/Schmidt 2013). Durch die Logit-Transformation wird erreicht, dass die Vorhersagewerte auf den Bereich zwischen 0 und 1 begrenzt sind (Best/Wolf 2010: 829ff.). Damit geht jedoch eine etwas kompliziertere Interpretation der Regressionskoeffizienten einher. Bei Längsschnittanalysen ist zudem die Interpretation als Effekte auf die Wahrscheinlichkeit problematisch (Brüderl 2010: 988). Hier liegt der Vorteil klar bei den linearen Modellen. Im linearen Wahrscheinlichkeitsmodell kann der Regressionskoeffizient als Veränderung der Wahrscheinlichkeit interpretiert werden, z.B. steht ein Koeffizient von 0,1 für eine Erhöhung der Wahrscheinlichkeit um 10 Prozentpunkte, dass Y zutrifft, wenn X um eine Einheit steigt.

Abbildung 4.4: Fiktives Beispiel: Lineares Wahrscheinlichkeitsmodell und Logit-Modell

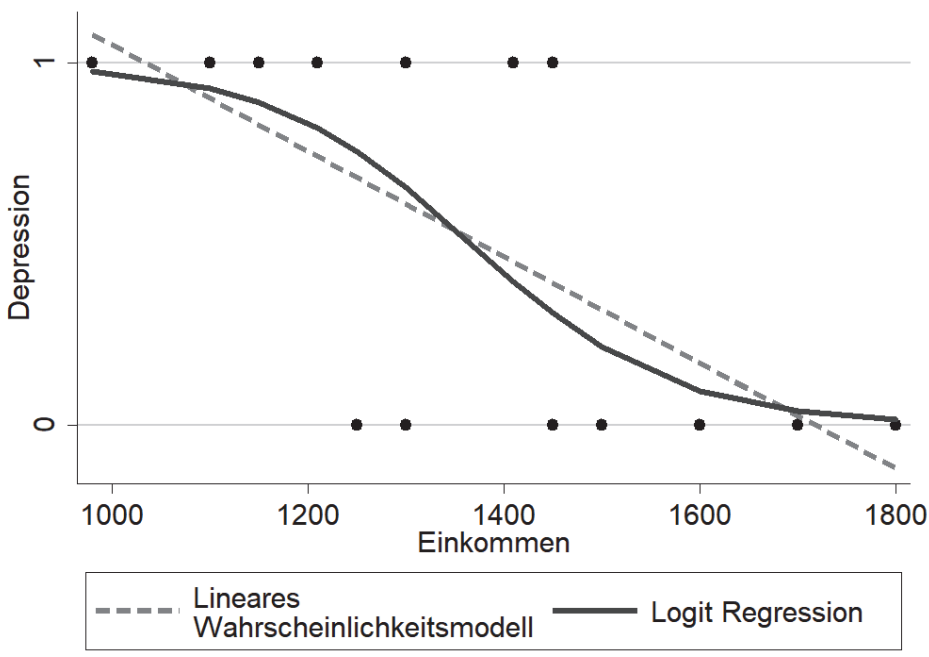

Quelle: Eigene Darstellung 
Mood (2010) beschreibt drei Probleme des linearen Wahrscheinlichkeitsmodells, die oft angeführt werden, um zu begründen, warum Logit- oder Probit-Modelle bei binären abhängigen Variablen die bessere Wahl sind (siehe auch Best/Wolf 2010). Erstes Argument ist, dass in den linearen Modellen die Vorhersagewerte der abhängigen Variablen nicht auf einen festgelegten Wertebereich begrenzt sind. Dies ist jedoch kein Problem, wenn bei der Interpretation der Ergebnisse der Bereich der plausiblen Werte (zwischen 0 und 1) interpretiert wird. Außerdem ist das kein Problem der binären Variablen, unrealistische Vorhersagewerte sind auch bei metrischen abhängigen Variablen möglich (Mood 2010). Als zweites Argument wird Heteroskedastizität genannt und dass die Residuen nicht normalverteilt sind, was zu Ineffizienz und invaliden Standardfehlern führt. Dies kann in linearen Modellen jedoch berücksichtigt werden, indem robuste Standardfehler berechnet werden (ebd.: 78). Das dritte Argument gegen die Verwendung eines linearen Modells ist, dass die Wahrscheinlichkeit nicht durch eine lineare Funktion beschrieben werden kann. Oft ist es theoretisch plausibel, dass eine binäre Variable nicht linear mit den unabhängigen Variablen verknüpft ist, sodass an den Enden der Verteilung die Änderung in der Wahrscheinlichkeit der abhängigen Variable geringer ist als in der Mitte (wie es mit dem Logit-Modell in Abbildung 4.4 dargestellt ist). Es ist aber durchaus möglich, dass ein linearer Zusammenhang besteht (vgl. Horrace/Oaxaca 2006). Letzten Endes ist die wahre Funktion unbekannt und ein Logit-Modell kann genauso wie ein lineares Modell eine unpassende Beschreibung der Realität darstellen (Schaffer 2012). Zudem wird oftmals eine nicht-lineare Funktionen ausreichend gut durch eine lineare Funktion beschrieben (ebd.).

Als Nachteile der nicht-linearen Regressionsmodelle können mehrere Punkte angebracht werden. Was Interaktionseffekte betrifft, ist eine einfache Interpretation in nicht-linearen Modellen - sowohl im Quer- als auch Längsschnitt - nicht möglich (Ai/Norton 2003). Ein weiterer Nachteil der Logit- und Probit-Modelle ist, dass bei ihnen die Koeffizienten nicht über verschiedene Berechnungen hinweg verglichen werden können (Best/Wolf 2010). Ein Vergleich wird möglich, indem man die durchschnittlichen marginalen Effekte (,average marginal effects“, AME) berechnet. Jedoch kann damit erstens nicht der nicht-lineare Zusammenhang beschrieben werden und zweitens gelangt man an diese auch mit dem linearen Wahrscheinlichkeitsmodell (Mood 2010). Wenn man nicht an der Nicht-Linearität, sondern nur dem AME interessiert ist, dann kann das lineare Wahrscheinlichkeitsmodell verwendet werden. Denn dessen Schätzer entsprechen den AME auf die Wahrscheinlichkeit für $\mathrm{y}=1$ (Mood 2010; Wooldridge 2002: 454). Da in dieser Arbeit Längsschnittanalysen durchgeführt werden, eignen sich die nicht-linearen Modelle aus einem weiteren Grund nicht als Analysemethode. Unter Verwendung der FixedEffect-Methode können ihre Schätzer verzerrt sein, vor allem bei begrenzten 
Beobachtungszeitpunkten und großer Fallzahl (,incidental parameters problem" siehe Greene 2002).

Die Frage, ob ein lineares oder nicht-lineares Wahrscheinlichkeitsmodell besser geeignet ist, wird diskutiert, wobei es Argumente für und gegen beide Varianten gibt (Horrace/Oaxaca 2006; Schaffer 2012; Lien/Rearden 1990). Während in ökonomischen Studien das lineare Wahrscheinlichkeitsmodell regelmäßig zum Einsatz kommt, ist es in der Soziologie eher selten (Mood 2010). Ausschlaggebend für die Entscheidung für lineare Wahrscheinlichkeitsmodelle in dieser Arbeit sind die Interpretierbarkeit der Koeffizienten und der Interaktionsterme sowie die Vergleichbarkeit zwischen Modellen, was insbesondere in den Längsschnittberechnungen ein Vorteil gegenüber der nicht-linearen Modelle darstellt. ${ }^{13}$

\subsubsection{Pooled-OLS}

In dieser Arbeit sollen u.a. Quer- und Längsschnittberechnungen verglichen werden (ausführliche Beschreibung bei Brüderl 2010). Dazu werden nun die Methoden vorgestellt, die hierfür typischerweise verwendet werden. Eine einfache Möglichkeit Paneldaten zu verwenden, ist das „Poolen“ der Daten, d.h. die Beobachtungen aus allen Zeitpunkten werden in einem Datensatz vereint. In dem resultierenden Datensatz haben Personen, die zu mehreren Zeitpunkten beobachtet wurden, entsprechend viele Datenzeilen. Mit diesen gepoolten Daten kann man dann Querschnitts-berechnungen durchführen, in diesem Fall eine lineare OLS (ordinary least square) Regression, die beschrieben wird mit:

$$
\begin{gathered}
y_{i t}=\alpha+x^{\prime}{ }_{i t} \beta+z_{i}^{\prime} \delta+u_{i t} \\
i=1, \ldots, N, t=1, \ldots, T
\end{gathered}
$$

Hierbei bezeichnet $i$ eine Person, $t$ den Zeitpunkt, $x_{i t}$ einen Vektor für alle unabhängigen zeitveränderlichen Variablen einer Person $i$ zum Zeitpunkt $t$ und $\beta$ den Vektor der entsprechenden Regressionskoeffizienten. $z_{i}$ ist der Vektor für alle unabhängigen zeitkonstanten Variablen einer Person $i$ und $\delta$ ist der Vektor der dazugehörigen Regressionskoeffizienten. ${ }^{14} u_{i t}$ bezeichnet den Fehlerterm $^{15}$. Diese Pooled-OLS-Methode (POLS) nutzt von den Paneldaten nur

13 Als Robustheitsanalyse werden in Kapitel 5.3 auch Längsschnitt-Logit Modelle (FE-Logit) berechnet.

14 Die Unterscheidung von $x_{i t}$ und $z_{i}$ ist hier nicht unbedingt notwendig, aber hilfreich für das Verständnis des Fixed-Effects-Modells, das später erläutert wird.

15 Der Fehlerterm unterscheidet sich vom Fehlerterm eines Querschnittmodells, da die Beobachtungen derselben Personen nicht unabhängig voneinander sind (Autokorrelation). Siehe auch Ausführung im Abschnitt „Random-Effects“. 
die erhöhte Fallzahl aus, so dass die Schätzer präziser werden. Ansonsten ist sie identisch mit einem OLS-Modell mit Querschnittdaten.

\subsubsection{Random-Effects}

Eine Möglichkeit die Informationen von Längsschnittdaten besser auszunutzen bietet das Random-Effects-Modell (RE-Modell). Für das RE-Modell wird der Fehlerterm in zwei Komponenten aufgeteilt:

$$
u_{i t}=\alpha_{i}+\varepsilon_{i t}
$$

Der Fehlerterm $\alpha_{\mathrm{i}}$ ist personenspezifisch und zeitkonstant, $\varepsilon_{\mathrm{it}}$ hingegen variiert über Personen und die Zeit hinweg (idiosynkratischer Fehler). Diese Zerlegung des Fehlers in einzelne Komponenten ist theoretisch auch mit Querschnittdaten möglich. Allerdings ist sie nur mit Längsschnittdaten umsetzbar, da hierfür zeitliche Variation in den Daten nötig ist. Ausgehend von Gleichung (2) kann das RE-Modell somit beschrieben werden mit:

$$
y_{i t}=x^{\prime}{ }_{i t} \beta+z^{\prime}{ }_{i} \delta+\alpha_{i}+\varepsilon_{i t}
$$

„Der Fehler ist in die beiden Komponenten zerlegt und die Konstante ist weggelassen, weil sie mit den personenspezifischen Fehlern kollinear wäre (man kann die $\alpha_{i}$ auch als personenspezifische Konstanten auffassen)“ (ebd.: 967). In diesem Modell besteht das Problem, dass die Fehler autokorreliert sind, d.h., dass die Beobachtungen einer Person zu den verschiedenen Zeitpunkten korrelieren. Beispielsweise ist das Einkommen einer Person stark mit deren Einkommen zu vorherigen Beobachtungszeitpunkten korreliert. Für OLS-Modelle ist die Autokorrelation ein Problem, dieses kann jedoch mit der Verwendung von Generalised Least Squares (GLS) behoben werden (die dazu notwendige Transformation (Feasible GLS) beschreibt (Brüderl 2010: 974)). Das RE-Modell ermöglicht es also ein POLS-Modell zu schätzen, bei dem die Panelstruktur der Daten (mehrere Zeitpunkte von derselben Person) berücksichtigt wird (Stein/Bekalarczyk 2017). Damit das RE-Modell zu validen Schätzern gelangt, müssen jedoch einige Annahmen erfüllt sein. So ist unter anderem erforderlich, „dass sowohl die personenspezifischen, wie auch die idiosynkratischen Fehler unabhängig, identisch verteilt sind und konstante Varianz haben“" (Brüderl 2010: 972). Sind diese Bedingungen nicht erfüllt, so gelangt das RE-Modell zu verzerrten Schätzern. 


\subsubsection{Fixed-Effects}

Auch das Fixed-Effects-Modell (FE-Modell) macht sich die Zerlegung des Fehlerterms zunutze, wobei es jedoch auf weniger Annahmen beruht als das RE-Modell. Im FE-Modell muss nur der idiosynkratischer Fehler $\varepsilon_{\text {it }}$ mit allen $\mathrm{X}$-Variablen des Modells unkorreliert sein, $\alpha_{\mathrm{i}}$ darf korrelieren. Ein weiterer Unterschied zum RE-Modell besteht darin, wie die Daten genutzt werden. Das RE-Modell nutzt - wie auch POLS - sowohl die Variation zwischen Personen (Between), als auch die Variation von denselben Personen im Zeitverlauf (Within). Das FE-Modell hingegen verwendet lediglich die Within-Variation. Diese Eigenschaften erlangt das FE-Modell durch eine Transformation. Zunächst ist die Between-Transformation notwendig. Hierbei wird das RE-Modell so verändert, dass nicht alle Beobachtungszeitpunkte einzeln, sondern pro Person die gemittelten Werte über alle Zeitpunkte, berücksichtigt werden.

$$
\bar{y}_{i}=\bar{x}_{i}^{\prime} \beta+z_{i}^{\prime} \delta+\alpha_{i}+\bar{\varepsilon}_{i}
$$

$\mathrm{z}_{\mathrm{i}}$ und $\alpha_{\mathrm{i}}$ sind konstant pro Person und müssen daher nicht gemittelt werden. Wendet man OLS auf die Daten nach dieser Transformation an, so erhält man den Between-Schätzer. Er verwendet ausschließlich die Variation zwischen Personen. Um zu dem FE-Modell zu gelangen muss die Within-Transformation durchgeführt werden. Hierbei wird Gleichung (5) von Gleichung (4) abgezogen, woraus folgende Gleichung resultiert:

$$
y_{i t}-\bar{y}_{i}=\left(x_{i t}-\bar{x}_{i}\right)^{\prime} \beta+\left(\varepsilon_{i t}-\bar{\varepsilon}_{i}\right)
$$

Wendet man OLS auf die Daten nach der Within-Transformation an, so erhält man den Within-Schätzer. Er verwendet ausschließlich die Variation über die Zeit. In die Berechnung gehen nur die Abweichungen vom persönlichen Mittelwert ein. FE-Modelle eignen sich daher um kausale Effekte zu untersuchen. Die zeitunveränderlichen Variablen $z_{i}$ sowie die personenspezifischen, unbeobachteten Fehler $\alpha_{i}$ sind nun eliminiert. Alle individuellen, zeitkonstanten Eigenschaften spielen keine Rolle. „Genau dies ist der große Vorteil von Paneldaten gegenüber Querschnittsdaten: Während eine Querschnittsregression einen Between-Schätzer liefert, der von personenspezifischer unbeobachteter Heterogenität verzerrt wird, liefert eine Panelregression (nach der WithinTransformation) einen Within-Schätzer, der von personenspezifischer unbeobachteter Heterogenität nicht verzerrt wird“ (ebd.: 968). Aus der Spezifikation des FE-Modells folgt aber auch, dass es keine Koeffizienten von zeitkonstanten Variablen liefert. Da diese keine Veränderungen aufweisen, können keine Effekte geschätzt werden. Dies wird oft als Nachteil betrachtet, sollte jedoch 
eher als Vorteil aufgefasst werden (ebd.: 975). Es sind keine Modelle mit vielen Variablen nötig und fehlende zeitkonstante Informationen stellen kein Problem dar, da auf sie ohnehin implizit kontrolliert werden.

Spielt personenspezifische Heterogenität keine Rolle, so ist die Anwendung eines RE-Modells angemessen und effizienter als die Berechnung eines FE-Modells. Um herauszufinden, welches Verfahren verwendet werden sollte, kann der Hausman-Test angewendet werden. Hierbei werden die Schätzer von RE- und FE-Modell verglichen. Bei identischen Schätzern ist das RE-Modellaufgrund der größeren Effizienz zu bevorzugen. Weichen die Schätzer stark voneinander ab, so sind die RE-Schätzer durch unbeobachtete Heterogenität verzerrt und die FE-Schätzer sind die bessere Wahl (Andreß/Golsch/Schmidt 2013: 168; Brüderl 2010: 976).

\subsubsection{First-Difference}

Eine Variation des Fixed-Effects-Modells ist das First-Difference-Modell (FD-Modell). Anstatt der Differenz vom personenspezifischen Mittelwert geht hierbei die Differenz zwischen zwei Zeitpunkten $t$ und $t-1$ in die Berechnungen ein, wie es durch folgende Gleichung beschrieben wird:

$$
y_{i t}-y_{i, t-1}=\left(x_{i t}-x_{i, t-1}\right)^{\prime} \beta+\left(\varepsilon_{i t}-\varepsilon_{i, t-1}\right)
$$

Durch den Vergleich von zwei aufeinanderfolgenden Zeitpunkten eignet sich diese Vorgehensweise, um kurzfristige bzw. schnell auftretende Effekte zu identifizieren. In Kapitel 6 wird es angewendet, um die Auswirkungen einer Veränderung in der finanziellen Situation zu untersuchen. Dazu wird es modifiziert, indem die Veränderung in der zentralen unabhängigen Variable - der finanziellen Situation - in Dummyvariablen umgewandelt wird. Jede mögliche Kombination der finanziellen Situation zu den Zeitpunkten $t$ und $t-1$ wird somit einzeln dargestellt. Alle anderen Variablen werden wie in Formel (7) dargestellt als Differenzen in das Modell aufgenommen.

Die Fallzahl in dem FD-Modell verringert sich im Vergleich zu den FEModellen aufgrund der Logik, die hinter dem Verfahren steckt. Die jeweils erste Beobachtung einer Person kann nicht verwendet werden, da es keine vorherige Beobachtung gibt, mit der eine Differenz gebildet werden kann. Um eine weitere größere Reduzierung der Fallzahl zu vermeiden, werden in den Analysen aufeinanderfolgende Beobachtungen berücksichtigt, die bis zu zwei Untersuchungswellen auseinanderliegen. Die Begrenzung der Stichprobe auf Beobachtungen, die aus direkt aufeinanderfolgenden Wellen stammen, würde zu einem erheblichen Verlust der Fallzahl führen. Viele Beobachtungen würden verloren gehen, da die dritte Welle von SHARE von den anderen Wellen 
abweicht und daher in dieser Arbeit gar nicht verwendet wird. Durch den maximalen Abstand von zwei Wellen, können alle Befragten, für die in Welle 2 und 4 Informationen vorliegen, in die Analysen integriert werden. Auch Personen, die aus anderen Gründen die Befragung eine Welle ausgesetzt haben, tragen so zu einem geringeren Rückgang der Stichprobengröße bei. Zudem gibt es einzelne Länder, die nicht in jeder Welle teilgenommen haben. Auch deshalb wird auf diese Weise eine größere Reduzierung der Fallzahl vermieden. Da Portugal nur in den Wellen 4 und 6 an SHARE teilgenommen hat, würde das Land ansonsten ganz aus den Analysen ausgeschlossen werden. Eine Begrenzung der Stichprobe auf Beobachtungen aus direkt aufeinanderfolgenden Wellen wird als Robustheitsanalyse durchgeführt (siehe Kapitel $6.2)$.

\subsubsection{Hybrid-Modell}

Ein Hybrid-Modell stellt eine Kombination aus RE- und FE-Modell dar (Allison 2009). Solch ein Modell ermöglicht es, neben den Within-Schätzern auch Effekte zeitkonstanter Variablen zu schätzen. Um dies zu erreichen wird ein RE-Modell geschätzt, wobei die zeitveränderlichen Variablen in jeweils zwei Variablen ,aufgeteilt" werden: Erstens als personenspezifischer Mittelwert (Between-Transformation) und zweitens bereinigt um den personenspezifischen Mittelwert (Within-Transformation). Das Modell wird durch folgende Gleichung beschrieben:

$$
y_{i t}=\left(x_{i t}-\bar{x}_{i}\right)^{\prime} \beta+\bar{x}_{i} \gamma+z_{i}^{\prime} \delta+\alpha_{i}+\varepsilon_{i t}
$$

Der Vorteil des Hybrid-Modells ist, dass die zeitkonstanten Variablen $z$ im Modell enthalten sind. Deren Schätzer $\delta$ sind im Gegensatz zu den Schätzern des RE-Modells nicht dadurch verzerrt, dass die zeitveränderlichen Variablen nur ungenügend kontrolliert werden (Brüderl 2010: 977). Die Within-Schätzer $\beta$ sind identisch mit den FE-Schätzern. $\gamma$ sind Between-Schätzer, die als „Niveaueffekt" der zeitveränderlichen Variablen betrachtet werden können. Brüderl weist jedoch darauf hin, dass die Between-Schätzer verzerrt sind, wenn unbeobachteter Heterogenität vorliegt und daher für die Identifikation von kausalen Zusammenhängen nicht von Interesse sind (ebd.). In dem Fall sind Between- und Within-Schätzer verschieden $(\beta \neq \gamma)$ und ein FE-Modell bzw. der Within-Schätzer sollte verwendet werden. Liegt keine personenspezifische Heterogenität vor, so ist die RE-Annahme erfüllt und Between- und WithinSchätzer sind gleich $(\beta=\gamma)$. Entweder ist also der Between-Schätzer verzerrt und daher sollte FE angewendet werden oder der Between-Schätzer ist unverzerrt und ein RE-Modell reicht für dessen Berechnung aus. Der Test $\beta=\gamma$ ist somit eine Alternative zum Hausman-Test. Während der Hausman-Test die 
Schätzer von RE und FE gegeneinander testet, wird im Hybrid Modell der Between- gegen den FE-Schätzer getestet (vgl. Andreß/Golsch/Schmidt 2013: 169).

Bei der vorliegenden Fragestellung und Datengrundlage ist ein HybridModell angebracht, da eine der interessierenden Variablen (Vermögensungleichheit), deren Effekt geschätzt werden soll, nicht zeitveränderlich vorliegt. Zudem sind von den zeitveränderlichen Variablen sowohl die Effekte der Veränderungen, als auch die Niveauunterschiede von Interesse. Dabei ist jedoch $\mathrm{zu}$ beachten, dass die Between-Schätzer nicht kausal interpretieren werden dürfen (Brüderl 2010: 973). 


\section{Analysen: Armut und Gesundheit}

Das erste Analysekapitel beschäftigt sich mit dem Zusammenhang von Armut - gemessen anhand finanzieller Probleme - und Gesundheit. Dazu werden im folgenden Abschnitt die zentralen Indikatoren deskriptiv analysiert um Länderunterschiede und Entwicklungen im Zeitverlauf deutlich zu machen und so den Rahmen für die folgenden Analysen und deren Interpretation zu setzten. Anschließend werden multivariate Analysen durchgeführt um Quer- und Längsschnittmethoden miteinander zu vergleichen. Zudem werden die Ergebnisse von Robustheitsanalysen vorgestellt. Die Interpretation und Diskussion der Ergebnisse bildet den Abschluss des Kapitels.

\subsection{Deskriptive Beschreibungen}

Bevor die multivariaten Analysen durchgeführt werden, werden in diesem Kapitel einige deskriptive Befunde berichtet. Hierzu wird zum einen die in Kapitel 4.5 beschriebene Stichprobe verwendet, zum anderen basieren manche der deskriptiven Analysen auf allen verfügbaren Fällen in SHARE, wobei diese gewichtet werden, um repräsentative Aussagen über die Bevölkerung ab 50 Jahren treffen zu können.

\subsubsection{Finanzielle Situation}

Abbildung 5.1 stellt für die untersuchten Länder dar, wie gut die Haushalte im Zeitverlauf finanziell über die Runden kommen. Dabei lassen sich einige Länder identifizieren, in denen die Mehrzahl der Haushalte leicht über die Runden kommt. In Dänemark, der Schweiz, Belgien und Luxemburg ist dies zu jedem Untersuchungszeitpunkt der Fall. Im Gegensatz dazu ist der Anteil der Haushalte, die überhaupt keine finanziellen Probleme berichten (Antwortkategorie „leicht") vor allem in Ost- und Südeuropa sehr gering. In Polen, Estland, sowie Italien, Griechenland und Portugal stellen diese Haushalte über den gesamten Beobachtungszeitraum die kleinste Gruppe dar. Neben diesem Muster im Niveau ist aus der Abbildung auch ersichtlich, dass in manchen Ländern im Zeitverlauf Veränderungen zu beobachten sind. Auffällig ist, dass in vielen Ländern der Anteil der Haushalte ohne finanzielle Probleme zugenommen hat. Da in Österreich, Deutschland, Schweden, Niederlande, Dänemark, Schweiz und Belgien gleichzeitig die Anteile der Haushalte mit einigen oder großen finanziellen Schwierigkeiten nahezu konstant geblieben sind, geht in diesen Ländern der höhere Anteil an Haushalten, die leicht über die Runden kommen, mit 


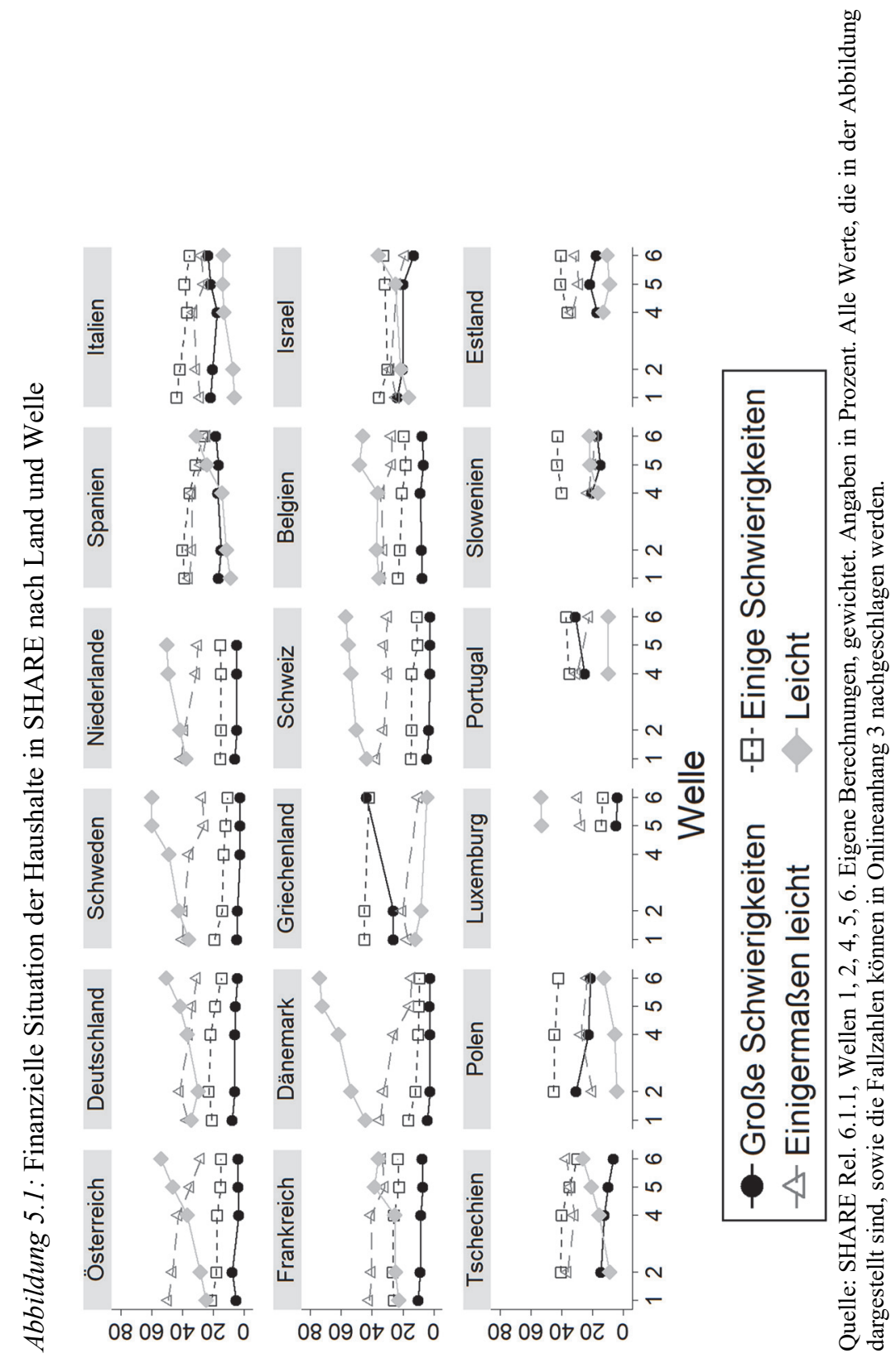




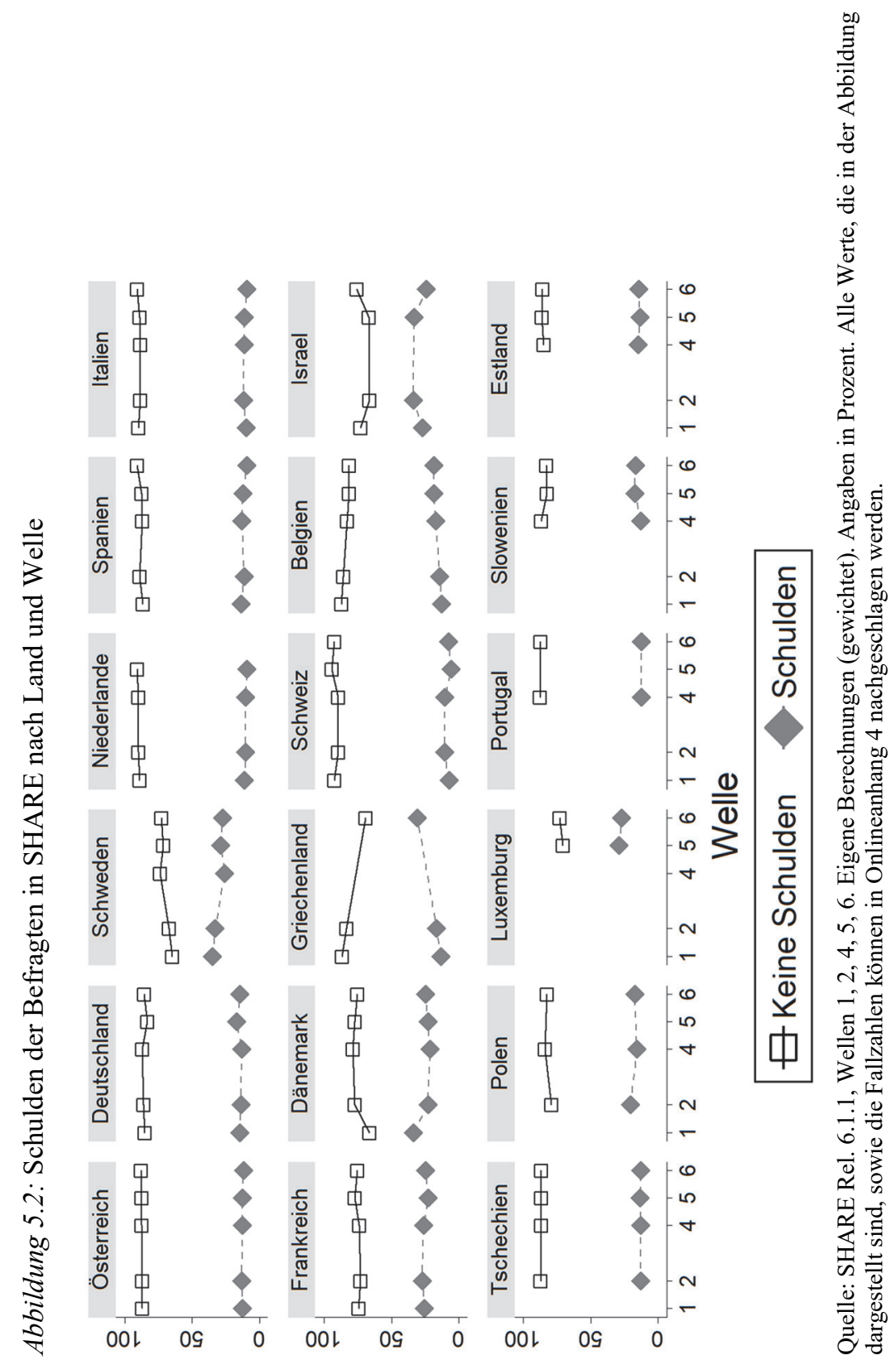


einer Reduzierung der Haushalte, die einigermaßen leicht über die Runden kommen, einher. Nur in Tschechien, Polen und Israel ist der Anteil der Haushalte mit finanziellen Schwierigkeiten deutlich zurückgegangen. Diese Übersicht macht deutlich, wie unterschiedlich die ökonomischen Bedingungen der Bevölkerung ab 50 Jahren in den europäischen Ländern sind. Der höchste Anteil von Haushalten ohne finanzielle Probleme liegt bei 74,1 Prozent in Dänemark, der niedrigste bei 4,4 Prozent in Griechenland, jeweils in Welle 6.

\subsubsection{Schulden}

Der Anteil der verschuldeten Personen pro Land und Untersuchungswelle ist in Abbildung 5.2 abgebildet. Es sind dabei teilweise deutliche Unterschiede zwischen den Ländern zu erkennen, die jedoch nicht die Situation der finanziellen Schwierigkeiten widerspiegeln. In Schweden ist der Anteil der Haushalte mit finanziellen Problemen relativ klein, der Anteil der Verschuldeten ist jedoch relativ hoch. Umgekehrt ist in manchen Ländern mit einem großen Anteil an Haushalten mit finanziellen Problemen, wie Spanien und Italien, eine Verschuldung eher selten. In den meisten Ländern ist der Anteil der Personen mit Schulden im Zeitverlauf relativ konstant. Die auffälligste Ausnahme stellt Griechenland dar. Hier stieg der Anteil der Verschuldeten im Untersuchungszeitraum von ca. 13,2 auf ca. 30,7 Prozent.

Tabelle 5.1: Anzahl depressiver Symptome in der Stichprobe (Welle 6)

\begin{tabular}{llrr} 
& $\begin{array}{l}\text { Anzahl } \\
\text { depressiver }\end{array}$ & & \\
& Symptome & Beobachtungen & $\%$ \\
\hline \multirow{4}{*}{ nicht depressiv } & 0 & 10.733 & 22,1 \\
& 1 & 10.293 & 21,2 \\
& 2 & 8.290 & 17,1 \\
& 3 & 6.377 & 13,2 \\
\hline & 4 & 4.632 & 9,6 \\
& 5 & 3.172 & 6,6 \\
depressiv & 6 & 2.120 & 4,4 \\
& 7 & 1.304 & 2,7 \\
& 8 & 808 & 1,7 \\
& 9 & 439 & 0,9 \\
& 10 & 200 & 0,4 \\
Summe & 11 & 80 & 0,2 \\
& 12 & 15 & 0,0 \\
\hline
\end{tabular}

Quelle: SHARE Rel. 6.1.1, Welle 6. Eigene Berechnungen, ungewichtet. 


\subsubsection{Depression}

Fast 40 Prozent der Befragten in SHARE Welle 6 geben an, im letzten Monat traurig oder niedergeschlagen gewesen zu sein. Damit ist Traurigkeit das am häufigsten genannte depressive Symptom unter den Befragten. Von Schlafstörungen und wenig Energie berichten jeweils mehr als ein Drittel der Befragten. Das Symptom, das die wenigsten Befragten angegeben haben (6,5 Prozent), ist der Wunsch tot zu sein (siehe Onlineanhang 5).

Betrachtet man die Anzahl depressiver Symptome, die von den Befragten angegeben wurden, so fällt die schiefe Verteilung auf. In Tabelle 5.1 sind die Werte von Welle 6 über alle Länder dargestellt. Jeweils über 20 Prozent der Befragten berichten kein oder nur ein Symptom. Sehr viele depressive Symptome (8 oder mehr) erreichen nur knapp 3 Prozent der Befragten. Bei Vorliegen von 4 oder mehr Symptomen gilt eine Person als depressiv (siehe Kapitel 4.2.1). In Welle 6 trifft dies auf 26,4 Prozent der Befragten zu. Die anderen 73,6 Prozent der Befragten werden als nicht depressiv eingestuft.

Abbildung 5.3: Prävalenz von Depression nach Land und Geschlecht (in Prozent, Welle 6)

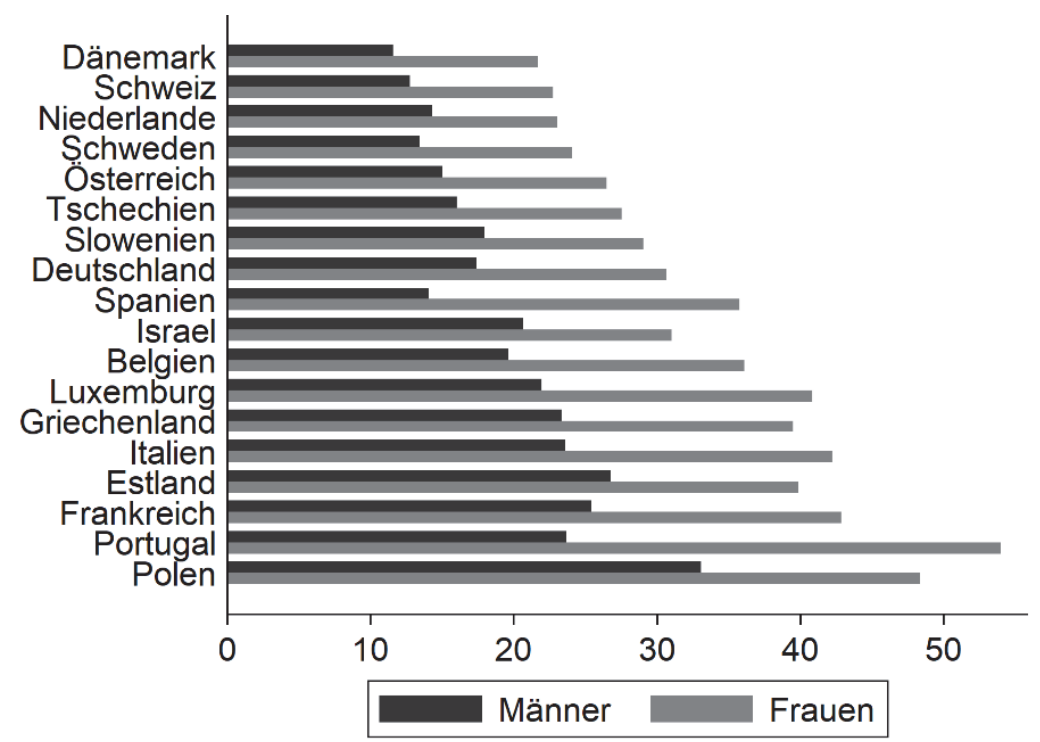

Quelle: SHARE Rel. 6.1.1, Welle 6 (außer Niederlande, Welle 5). Eigene Berechnungen, gewichtet. Sortierung der Länder nach dem Gesamtdurchschnitt. Alle Werte, die in der Abbildung dargestellt sind, sowie die Fallzahlen können in Onlineanhang 6 nachgeschlagen werden. 
Die bekannten Unterschiede zwischen Frauen und Männern in der Prävalenz von Depression (siehe Kapitel 2.3.5) werden auch anhand der SHARE-Daten bestätigt. In Abbildung 5.3 ist prozentual dargestellt wie viele Frauen und Männer in den einzelnen Ländern als depressiv eingestuft werden. In jedem Land ist der Anteil der depressiven Personen bei Frauen deutlich höher als bei Männern. Besonders ins Auge sticht Portugal, da hier jede zweite Frau als depressiv gilt. Der geringste Unterschied zwischen Männern und Frauen ist mit 8,8 Prozentpunkten in den Niederlanden vorhanden. In Portugal ist der Unterschied mit 30,3 Prozentpunkten am höchsten. Auch bei der Prävalenz von Depression können beachtliche Länderunterschiede beobachtet werden. Dänemark weist mit nur 16,9 Prozent den geringsten, Polen mit 41,6 Prozent den höchsten Wert im Durchschnitt beider Geschlechter auf. Bei den Befragten aus Deutschland werden 24,5 Prozent der Fälle als depressiv eingestuft (vgl. Onlineanhang 6).

Ein weiterer Befund bisheriger Studien, der hier repliziert werden kann, ist eine mit dem Alter ansteigende Wahrscheinlichkeit an einer Depression zu leiden (siehe Abbildung 5.4). Ab 75 Jahren leidet demnach über ein Drittel der Bevölkerung in Europa an Depressionen. Im Alter von 90 bis 94 Jahren liegt der Anteil sogar bei 47,1 Prozent.

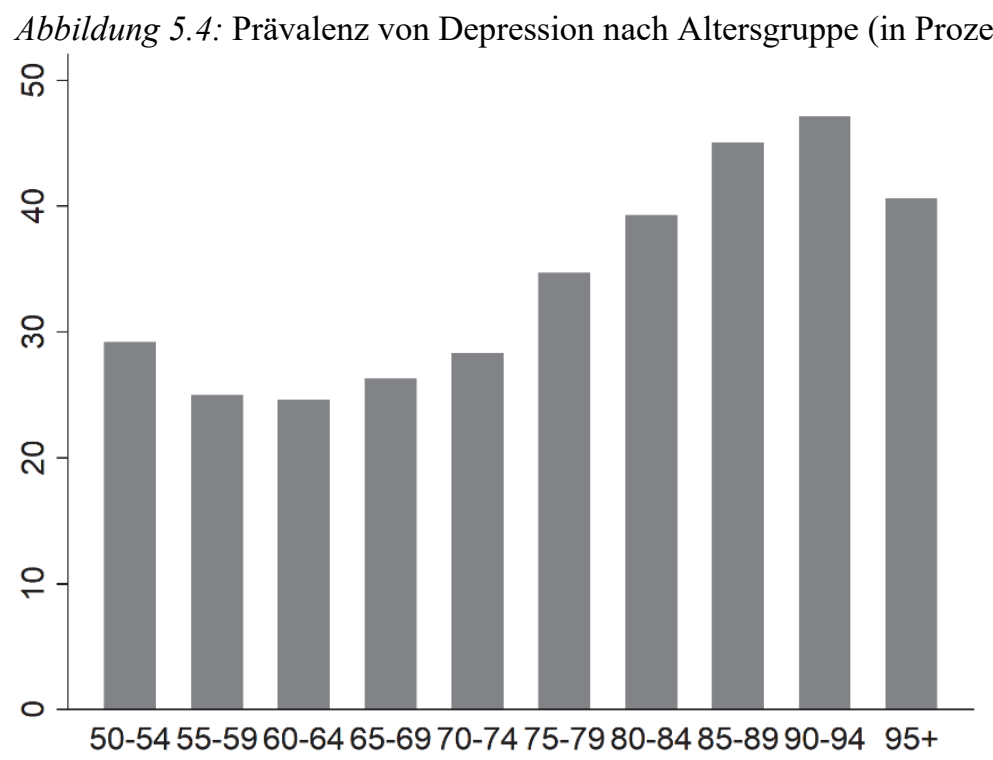

Quelle: SHARE Rel. 6.1.1, Welle 6. (außer Niederlande, Welle 5). Eigene Berechnungen, gewichtet. Angaben in Prozent. Alle Werte, die in der Abbildung dargestellt sind, können in Onlineanhang 7 nachgeschlagen werden. 
Die Länderunterschiede und Entwicklungen der Prävalenz von Depressionen im Beobachtungszeitraum sind in Abbildung 5.5 dargestellt. Mit Ausnahme von Belgien ist in allen Ländern zwischen Welle 1 und 2 ein Rückgang der Depressionsrate zu beobachten. Zwischen den Wellen 2 und 4 überwiegt ein Anstieg in der Prävalenz von Depressionen. Nur in Italien, Dänemark und Polen ist in diesem Zeitraum der Anteil depressiver Personen im Alter ab 50 Jahren rückläufig. Im weiteren Verlauf, ab Welle 4, lassen sich keine klaren Muster erkennen. Über den gesamten Untersuchungszeitraum können zum Teil gegenläufige Entwicklungen in der Depressionsrate beobachtet werden. Während in Griechenland der Anteil depressiver, älterer Menschen stark ansteigt, ist in Spanien und Israel eine deutliche Abnahme der Depressionsprävalenz zu sehen.

Abbildung 5.5: Prävalenz von Depression im Zeitverlauf nach Land (in Prozent)

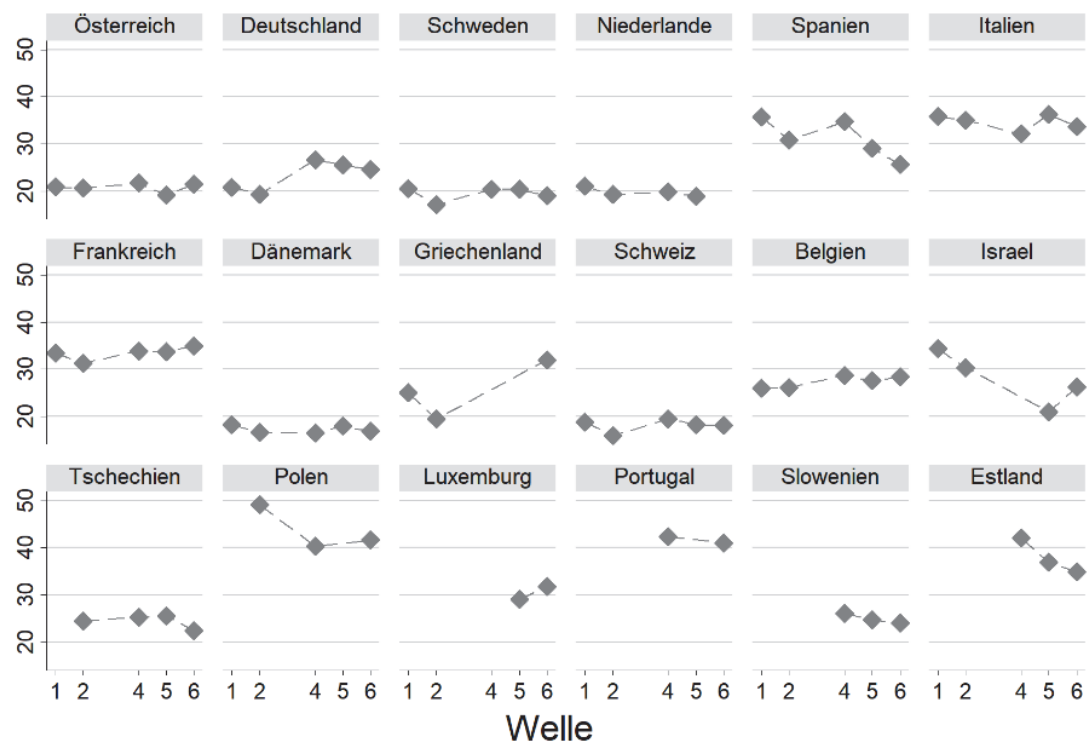

Quelle: SHARE Rel. 6.1.1, Wellen 1, 2, 4, 5, 6. Eigene Berechnungen, gewichtet. Angaben in Prozent. Alle Werte, die in der Abbildung dargestellt sind, sowie die Fallzahlen können in Onlineanhang 8 nachgeschlagen werden. 


\subsubsection{Einschränkungen in den Aktivitäten des täglichen Lebens}

Die Mehrheit der Personen in der Stichprobe hat keine Einschränkung in Aktivitäten des täglichen Lebens (ADL) angegeben. In SHARE trifft dies in Welle 6 auf 78,1 Prozent der Befragten zu. Von den abgefragten Aktivitäten ist die Haus- und Gartenarbeit diejenige, bei der die Befragten am häufigsten von Schwierigkeiten berichten - in 12,7 Prozent der Fälle. Alle anderen Aktivitäten werden von weniger als 9 Prozent der Befragten angegeben. Es berichten bspw. 8,5 Prozent von Problemen mit dem Anziehen, 4,8 Prozent beim Zubereiten einer warmen Mahlzeit und ca. 2,9 Prozent beim Benutzen der Toilette (siehe Onlineanhang 9).

Einschränkungen in Aktivitäten hängen stark mit dem Alter zusammen (siehe Abbildung 5.6). Während von den 50-54 jährigen unter 10 Prozent von einer oder mehreren Einschränkungen berichten, steigt der Anteil von Personen mit Einschränkungen mit höherem Alter an. Zunächst ist der Anstieg eher gering, bis 14,3 Prozent in der Gruppe der 65-69 jährigen. Ab 70 Jahren fällt der Anstieg deutlich stärker aus, der Anteil steigt auf 59,2 Prozent im Alter

Abbildung 5.6: Einschränkungen in Aktivitäten des täglichen Lebens nach Altersgruppe (in Prozent)

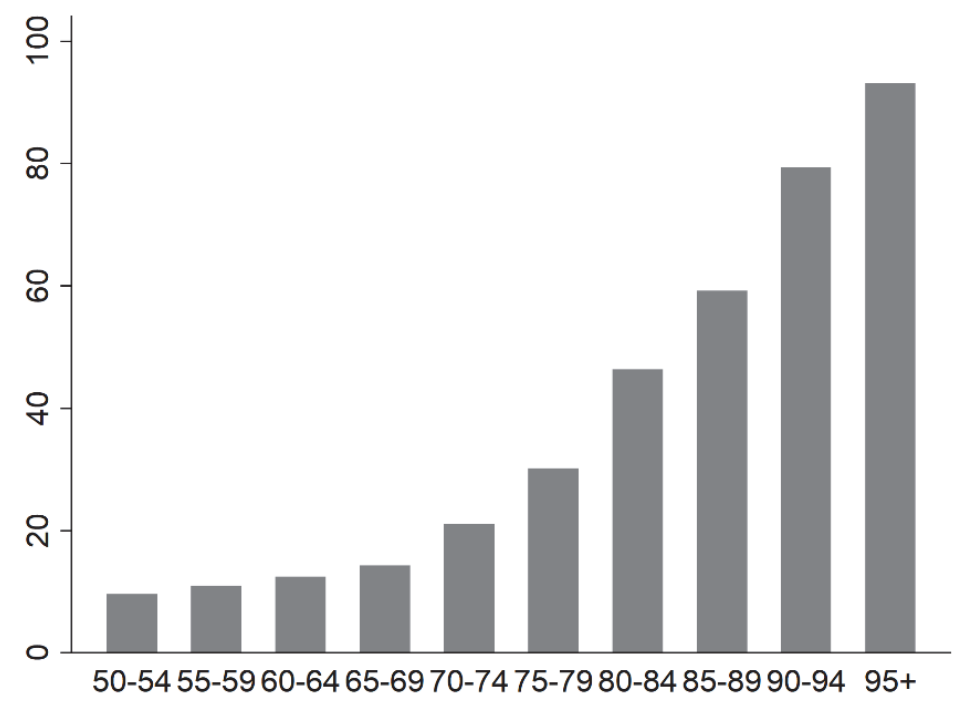

Quelle: SHARE Rel. 6.1.1, Welle 6. (außer Niederlande, Welle 5). Eigene Berechnungen, gewichtet. Angaben in Prozent. Alle Werte, die in der Abbildung dargestellt sind, können in Onlineanhang 10 nachgeschlagen werden. 
Abbildung 5.7: Einschränkungen in Aktivitäten des täglichen Lebens im Zeitverlauf nach Land (in Prozent)

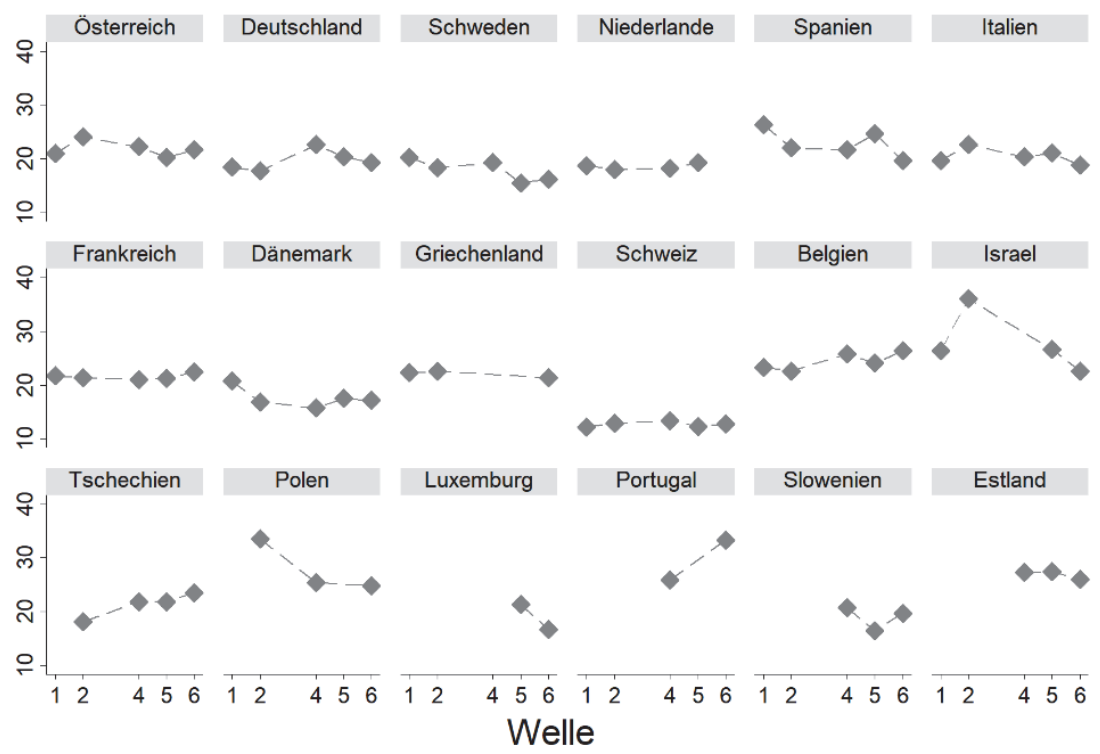

Quelle: SHARE Rel. 6.1.1, Wellen 1, 2, 4, 5, 6. Eigene Berechnungen, gewichtet. Angaben in Prozent. Alle Werte, die in der Abbildung dargestellt sind, sowie die Fallzahlen können in Onlineanhang 11 nachgeschlagen werden.

von 85 bis 90 Jahren. Ab 95 Jahren berichten fast alle Befragten (93,1 Prozent) von mindestens einer Einschränkung.

In Abbildung 5.7 sind die Anteile der Personen mit Einschränkungen in ADL in der Bevölkerung ab 50 Jahren über alle Länder und Beobachtungszeitpunkte dargestellt. Auch hier lassen sich zum Teil große Unterschiede zwischen den Ländern beobachten. Mit Werten zwischen 12,2 und 13,4 Prozent ist die Schweiz das Land mit dem geringsten Anteil an Personen mit körperlichen Einschränkungen. Für die meisten Länder liegen die Anteile zwischen 20 und 25 Prozent, wobei es auch Ausreißer nach oben gibt - insbesondere in Polen, Portugal und Israel. Deutliche Trends für eine Entwicklung im Zeitverlauf sind nicht erkennbar. 


\subsubsection{Schlechte subjektive Gesundheit}

Betrachtet man die Anteile der Personen ab 50 Jahren, die eine schlechte subjektive Gesundheit angeben, so fällt auf, dass diese im Zeitverlauf recht stabil sind (siehe Abbildung 5.8). Nur in Polen und Israel lässt sich ein deutlicher Trend erkennen: In diesen Ländern sank im Zeitverlauf der Anteil an Personen mit schlechter Gesundheit. Die Länderunterschiede im Niveau deuten auf ein ähnliches Muster wie bei den anderen Gesundheitsmaßen hin. Portugal, Polen und Estland sind die Länder mit dem höchsten Anteil an Personen mit schlechter subjektiver Gesundheit. In Estland ist der Anteil besonders hoch, der Wert liegt konstant um die 70 Prozent. In Dänemark, der Schweiz und Belgien ist schlechte subjektive Gesundheit am wenigsten verbreitet. In der Schweiz liegen die Werte zu jedem Beobachtungszeitpunkt unter 20 Prozent.

Abbildung 5.8: Schlechte subjektive Gesundheit im Zeitverlauf nach Land (in Prozent)

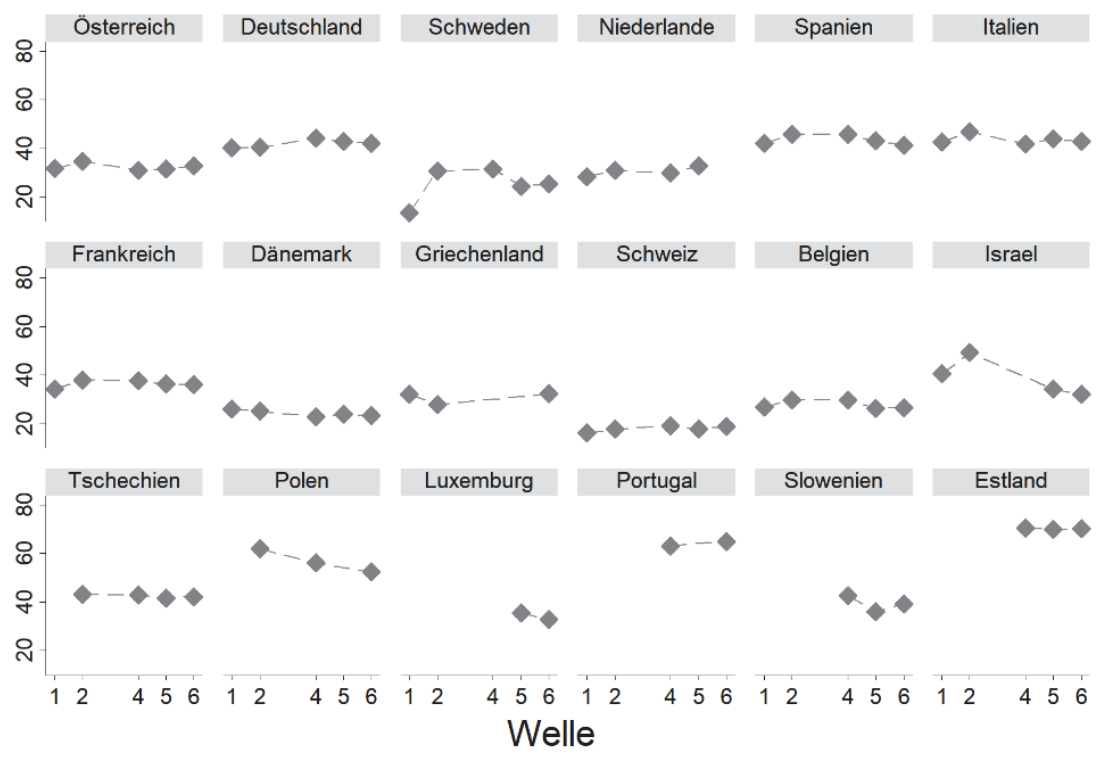

Quelle: SHARE Rel. 6.1.1, Wellen 1, 2, 4, 5, 6. Eigene Berechnungen, gewichtet. Angaben in Prozent. Alle Werte, die in der Abbildung dargestellt sind, sowie die Fallzahlen können in Onlineanhang 12 nachgeschlagen werden.

Auch bei der subjektiven Gesundheit ist ein deutlicher Zusammenhang mit dem Alter erkennbar. Während in der Gruppe der 50-54 jährigen nur 26,9 Pro- 
zent eine schlechte subjektive Gesundheit berichten, steigt dieser Anteil kontinuierlich an bis auf einen Höchstwert von 71,5 Prozent im Alter von 90 bis 94 Jahren (die Werte beziehen sich auf SHARE Welle 6, siehe Onlineanhang 13).

\subsubsection{Bivariate Analysen}

Um einen ersten Eindruck für den Zusammenhang von finanzieller Situation und Gesundheit zu bekommen, lohnt sich ein Blick auf bivariate Analysen. Abbildung 5.9 verdeutlicht wie stark die finanzielle Lage des Haushaltes mit Depression verknüpft ist. In allen Beobachtungswellen zeigt sich dasselbe Muster. Mit größeren finanziellen Problemen geht ein höherer Anteil an Personen mit Depressionen einher. In Haushalten, die nur mit großen Schwierigkeiten über die Runden kommen, gilt fast jede zweite Person als depressiv. In Haushalten, die leicht über die Runden kommen, gilt dies für weniger als jede fünfte Person. Zwischen Personen, die in Haushalten mit großen Schwierigkeiten leben und Personen, die in Haushalten mit einigen Schwierigkeiten leben, ist der Abstand in der Depressionsprävalenz mit 14 bis 16 Prozentpunkten

Abbildung 5.9: Anteil der Personen mit Depression nach finanzieller Situation

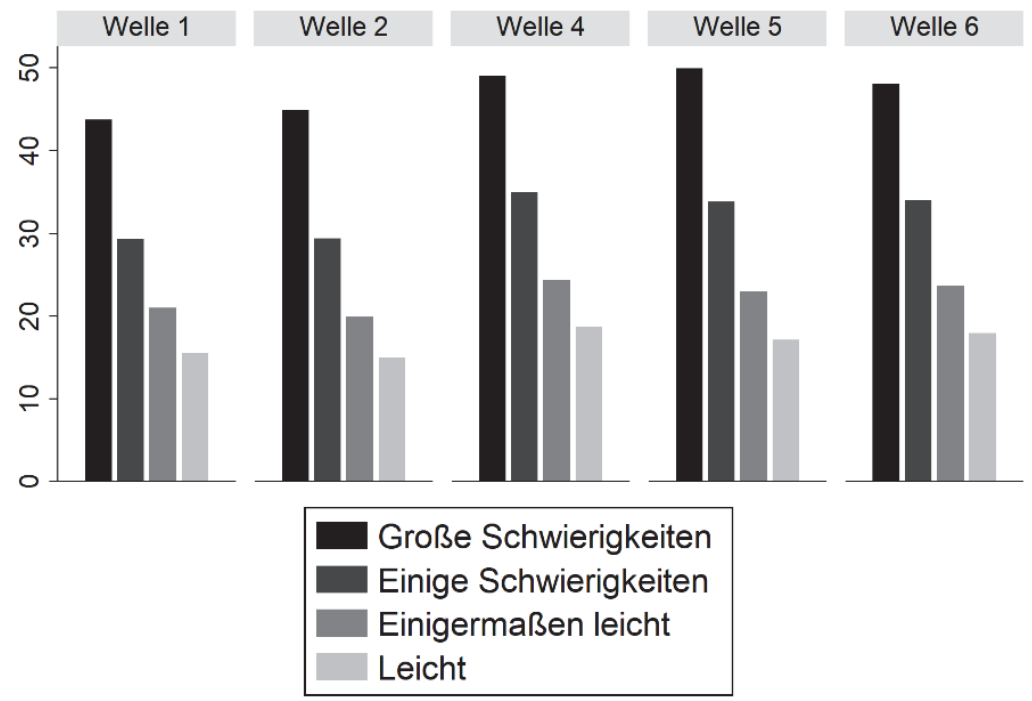

Quelle: SHARE Rel. 6.1.1, Wellen 1, 2, 4, 5, 6. Eigene Berechnungen, ungewichtet. Angaben in Prozent. Alle Werte, die in der Abbildung dargestellt sind, sowie die Fallzahlen können in Onlineanhang 15 nachgeschlagen werden. 
besonders groß. Zwischen den Kategorien „einigermaßen leicht“ und „leicht“ liegen die Unterschiede hingegen nur bei 5 bis 6 Prozentpunkten.

Zwischen den Ländern existieren zwar die bereits genannten Unterschiede im Niveau der Prävalenz von Depression, allerdings findet man in allen Ländern, dass Personen in Haushalten mit finanziellen Problemen öfter an Depression leiden als Personen, die in Haushalten ohne finanzielle Schwierigkeiten leben. In manchen Ländern ist der Anteil depressiver Personen in Haushalten der Kategorie „leicht" jedoch etwa gleich hoch oder sogar höher als in der Kategorie ,einigermaßen leicht“. In Welle 6 ist dies in Portugal, Polen, Italien und Griechenland der Fall (siehe Onlineanhang 14).

Das Muster aus Abbildung 5.9, zeigt sich auch für Einschränkungen in Aktivitäten des täglichen Lebens (siehe Onlineanhang 16) sowie für schlechte subjektive Gesundheit (siehe Onlineanhang 17). Während dies für die subjektive Gesundheit über alle Beobachtungswellen und Länder zu sehen ist, ist das

Abbildung 5.10: Depression, Einschränkungen in ADL und schlechte subjektive Gesundheit nach Schulden und Welle

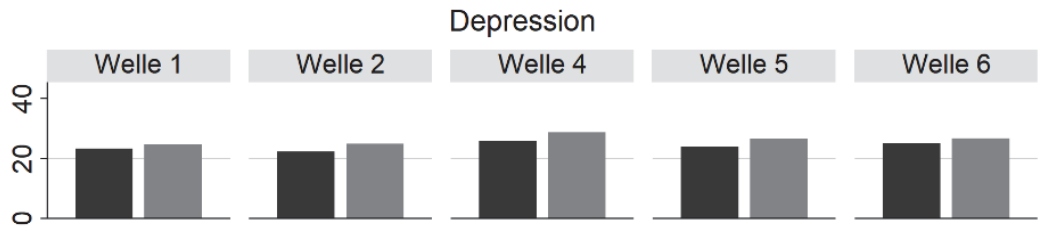

Einschränkungen in ADL

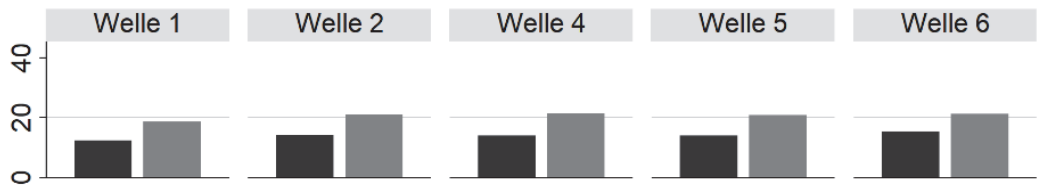

Schlechte subjektive Gesundheit

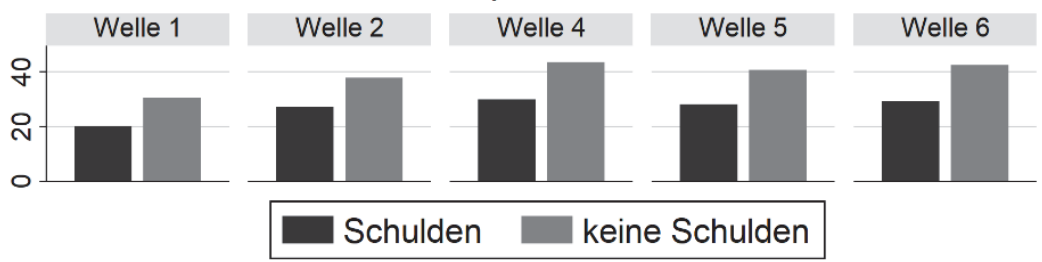

Quelle: SHARE Rel. 6.1.1, Wellen 1, 2, 4, 5, 6. Eigene Berechnungen, ungewichtet. Angaben in Prozent. Alle Werte, die in der Abbildung dargestellt sind, sowie die Fallzahlen können in Onlineanhang 19 nachgeschlagen werden. 
Bild etwas uneinheitlicher für die Einschränkungen in den ADL. Die größten Ausreiser sind Luxemburg in Welle 5 und Polen in Welle 4. In Luxemburg ist der geringste Anteil an Personen mit Einschränkungen in ADL in der Gruppe mit den größten finanziellen Problemen und in Polen ist in Haushalten ohne finanzielle Sorgen die Wahrscheinlichkeit einer Einschränkung deutlich höherer als in Haushalten mit einigen finanziellen Schwierigkeiten.

Ein weiterer Indikator der finanziellen Lage eines Haushaltes soll hier noch in der bivariaten Analyse betrachtet werden: Schulden. Die Prävalenz von Depression, Einschränkungen in Aktivitäten und schlechter subjektiver Gesundheit ist in Haushalten mit Schulden geringer als in Haushalten ohne Schulden (siehe Abbildung 5.10). Dieses Ergebnis lässt sich in allen Wellen von SHARE finden. Da Schulden ein psychologischer Stressfaktor sein können (vgl. Kapitel 4.1.2), wäre genau das Gegenteil zu erwarten. Das Muster zeigt sich jedoch für die subjektive Gesundheit und die Einschränkungen in Aktivitäten in fast allen Ländern. Für Depression hingegen ist in einigen Ländern der vermutete, umgekehrte Zusammenhang zu beobachten. Am häufigsten kann in Welle 5 beobachtet werden, dass Depressionen in Haushalten mit Schulden häufiger vorkommen, als in Haushalten ohne Schulden, z. B. in Deutschland, Spanien, Italien, Belgien, Tschechien und Luxemburg (siehe Onlineanhang 18).

\subsection{Vergleich von Querschnitt- und Längsschnittanalysen}

Um den Zusammenhang von finanzieller Lage und Gesundheit zu bestimmen, werden lineare Wahrscheinlichkeitsmodelle mit verschiedenen Methoden berechnet. Ein OLS-Modell, das nur Daten von einem Beobachtungszeitpunkt (Welle 6) verwendet, dient als Referenz, da dies die am häufigsten verwendete Methode in der bisherigen Forschung ist. Die anderen Methoden - PooledOLS (POLS), Random-Effects (RE) und Fixed-Effects (FE) - berücksichtigen die Daten aller Wellen auf unterschiedliche Weise (siehe Kapitel 4.6). In allen Modellen wird für Alter, Partner im Haushalt, Beobachtungswelle, Erwerbsstatus, Schulden, Miete, Erbe, Geschenke und die Person, die die finanziellen Fragen des Haushaltes beantwortet hat, kontrolliert. Im OLS-, POLS- und REModell sind zusätzlich Variablen für das Geschlecht, die Bildung und das Land enthalten. Zudem werden in den Berechnungen mit Depression als abhängiger Variable die Einschränkungen in ADL und subjektive Gesundheit kontrolliert. Umgekehrt wird in Analysen der Einschränkungen in den ADL und subjektiven Gesundheit für Depression kontrolliert. Alle Berechnungen werden ungewichtet und mit panel-robusten Standardfehlern durchgeführt (ebd.). 
In Abbildung 5.11 sind die Regressionskoeffizienten für die finanzielle Lage aus allen vier Modellen mit Depression als abhängiger Variable dargestellt. Referenzkategorie ist hierbei die Gruppe der Haushalte ohne finanzielle Probleme (Kategorie „leicht“). Die Koeffizienten entsprechen den durchschnittlichen marginalen Effekten und können als Änderungen in Prozentpunkten interpretiert werden. Es ist das gleiche Muster wie in der bivariaten Analyse zu erkennen. Mit größeren finanziellen Schwierigkeiten geht eine höhere Depressionswahrscheinlichkeit einher. Zwischen den Modellen bestehen jedoch Unterschiede in den Effektstärken. Die Ergebnisse von POLS sind denen der Querschnittberechnung mit Welle $6 \mathrm{sehr}$ ähnlich. Dies ist nicht verwunderlich, denn der Unterschied liegt lediglich in der Anzahl der Fälle, die für die Berechnungen verwendet werden. Unter Berücksichtigung aller Wellen sind daher genauere Schätzer zu erwarten, was sich in den kleineren Konfidenzintervallen bei POLS widerspiegelt.

\section{Abbildung 5.11: Vergleich von OLS, POLS, RE und FE - Depression}

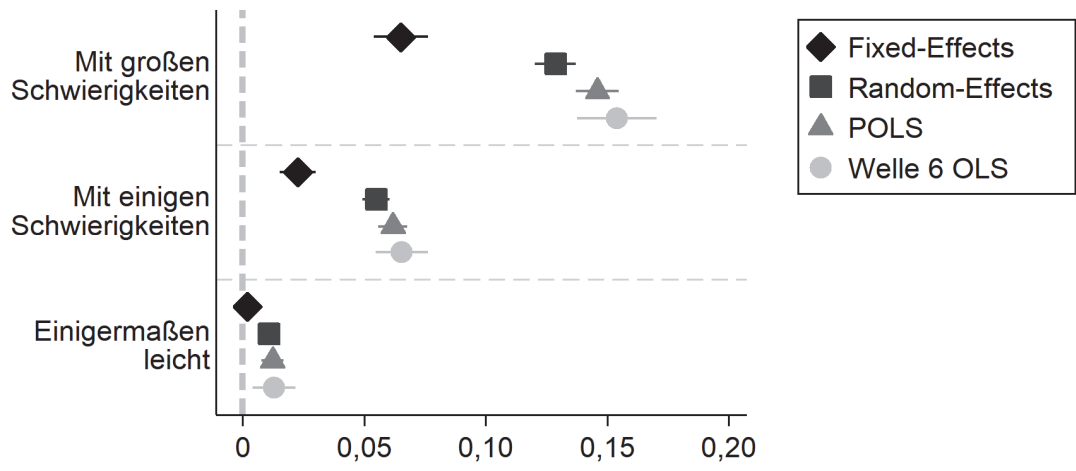

Quelle: SHARE Rel. 6.1.1, Wellen 1, 2, 4, 5, 6. Eigene Berechnungen. Darstellung der Koeffizienten und 95\% Konfidenzintervalle. Die komplette Regressionstabelle befindet sich in Onlineanhang 20.

Bei POLS ist die Wahrscheinlichkeit bei großen finanziellen Problemen depressiv zu sein um 14,6 Prozentpunkte höher als in der Referenzkategorie „leicht“. Im RE-Modell liegt der Unterschied etwas niedriger bei 12,9, im FEModell nur bei 6,5 Prozentpunkten. Auch bei den Koeffizienten für ,einige Schwierigkeiten“ und „einigermaßen leicht“ lässt sich dieses Muster erkennen. Daraus lässt sich schließen, dass ein Großteil des Zusammenhangs, der im Querschnitt beobachtet werden kann, auf individuelle Eigenschaften zurückzuführen ist, die in den Querschnittberechnungen nicht berücksichtigt werden. Im FE-Modell sind hingegen alle personenspezifischen zeitkonstanten Eigenschaften kontrolliert. Es gibt darüber Auskunft wie sich eine Änderung in der 
finanziellen Situation auf das Depressionsrisiko auswirkt. Der Wechsel von keinen zu großen finanziellen Problemen ist also mit einer Steigerung des Depressionsrisikos um 6,6 Prozentpunkte verbunden. Dies entspricht in etwa der gleichen Effektstärke wie der Wechsel vom Zusammenleben mit einem Partner zu keinem Partner im Haushalt (6,1 Prozentpunkte, siehe Onlineanhang 20). Deutlich geringer (2,3 Prozentpunkte) ist der Unterschied im Depressionsrisiko bei einem Wechsel von keinen zu einigen finanziellen Problemen. Bei dem POLS und dem RE-Modell sind kleine aber signifikante Unterschiede in der Wahrscheinlichkeit einer Depression zwischen den Kategorien „leicht“ und ,einigermaßen leicht“ zu finden. Bei der FE-Berechnung hingegen zeigt sich hier kein signifikanter Zusammenhang.

Die beschriebenen Muster lassen sich auch bei der Analyse von Einschränkungen in ADL sowie der subjektiven Gesundheit finden (siehe Abbildung 5.12). Die Effektstärken bei OLS, POLS und RE sind bei den Einschränkungen in ADL etwas geringer als bei den beiden anderen Gesundheitsindikatoren. Im FE-Modell zeigen sich ähnliche Effekte für Einschränkungen in den ADL und

Abbildung 5.12: Vergleich von OLS, POLS, RE und FE - Einschränkungen in ADL und subjektive Gesundheit
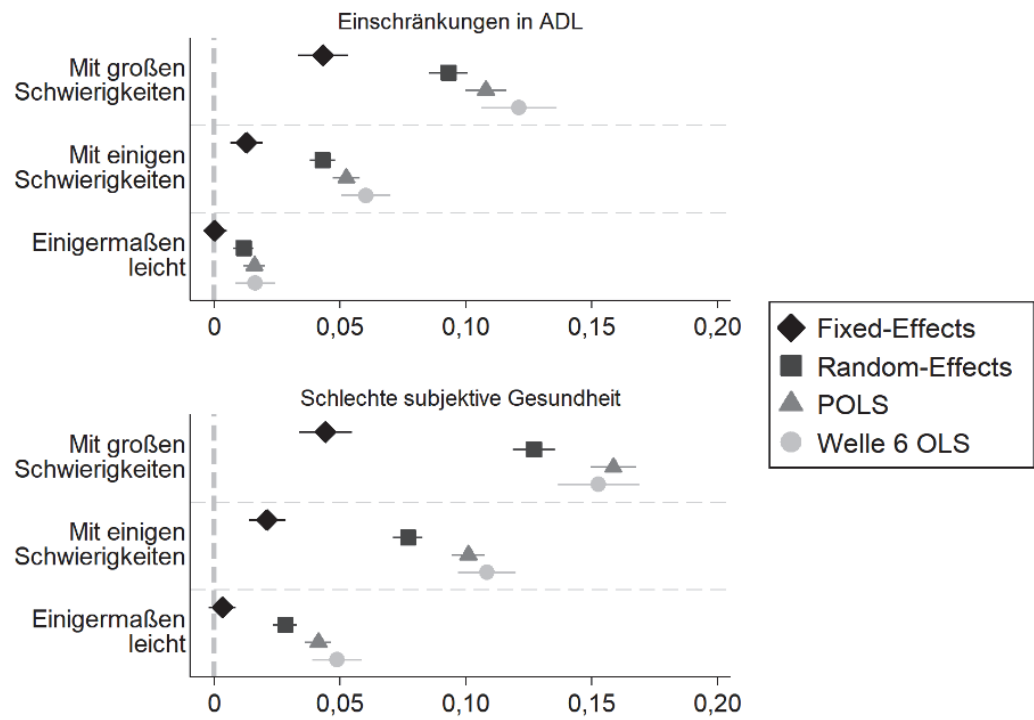

Quelle: SHARE Rel. 6.1.1, Wellen 1, 2, 4, 5, 6. Eigene Berechnungen. Darstellung der Koeffizienten und 95\%-Konfidenzintervalle. Die komplette Regressionstabelle befindet sich in Onlineanhang 21. 
subjektive Gesundheit. Für beide Indikatoren erhöht sich die Wahrscheinlichkeit für einen schlechten Gesundheitszustand bei einem Wechsel zu großen finanziellen Schwierigkeiten um ca. 4 Prozentpunkte. Bei einigen Schwierigkeiten beträgt der Effekt nur 1 Prozentpunkt bei Einschränkungen in ADL und 2 Prozentpunkte bei schlechter subjektiver Gesundheit. Dies entspricht jeweils ca. derselben Effektstärke, die mit einem 2 Jahre höherem Alter verbunden ist.

Die Unterschiede in den Schätzern zwischen FE und den anderen Methoden deuten darauf hin, dass die Schätzer von OLS, POLS und RE durch personenspezifische Heterogenität verzerrt werden. Dies kann mit dem HausmanTest überprüft werden. Hierbei wird ein RE-Modell und ein FE-Modell miteinander verglichen, indem die standardisierte Differenz der Parameterschätzer der beiden Modelle berechnet wird (ebd.: 976). Bei einer geringen Differenz kann ein RE-Modell, bei großer Differenz hingegen ein FE-Modell angewendet werden. Bei allen drei abhängigen Variablen ist das Ergebnis des Hausman-Tests eindeutig: Die Schätzer von RE- und FE-Modellen weichen stark voneinander $a b$, d.h. es liegt personenspezifische Heterogenität vor, daher sollte das FE-Modell verwendet werden.

Wie oben dargestellt wurde, können in den FE-Berechnungen bei allen untersuchten Gesundheitsindikatoren zwischen den beiden Kategorien mit geringen und keinen finanziellen Sorgen (,einigermaßen leicht" und ,leicht") keine signifikanten Unterschiede festgestellt werden. Daher werden diese beiden Kategorien in den weiteren Analysen zusammengefasst. Dass hierdurch die Schätzer der beiden anderen Kategorien kaum beeinflusst werden, zeigt Abbildung 5.13. Hier sind die Schätzer aus insgesamt sechs Berechnungen -für die drei Gesundheitsindikatoren in jeweils zwei Varianten - dargestellt. Im oberen Teil ist die finanzielle Situation in vier Kategorien eingeteilt (Referenz „leicht“", entspricht den Abbildungen 5.11 und 5.12). Im unteren Teil der Abbildung sind die Ergebnisse bei einer Einteilung in drei Kategorien dargestellt (Referenz ,einigermaßen leicht oder leicht"). Auch die Koeffizienten der Kontrollvariablen werden durch die Zusammenlegung der beiden Kategorien nicht beeinflusst (siehe Onlineanhang 22).

Neben der subjektiv eingeschätzten finanziellen Situation enthalten die Berechnungen weitere Variablen, die die finanzielle Lage des Haushaltes beschreiben. Für alle untersuchten Gesundheitsindikatoren stellt Verschuldung einen Risikofaktor dar. Hierbei ist die Effektstärke in den FE-Modellen mit der Effektstärke von einigen finanziellen Schwierigkeiten vergleichbar. Schulden erhöhen das Risiko an Depression zu leiden um 2,9 Prozentpunkte und die Wahrscheinlichkeit von Einschränkungen in Aktivitäten um 2,1 Prozentpunkte. Für schlechte subjektive Gesundheit liegt der Effekt bei 1,6 Prozentpunkten (siehe Onlineanhang 22). Ob der Haushalt Miete zahlen muss oder nicht ist hingegen in den FE-Modellen nur mit Depression verknüpft. Mit einer 
Abbildung 5.13: Vergleich von FE-Modellen mit unterschiedlicher Referenzkategorie der Variable „finanzielle Probleme“
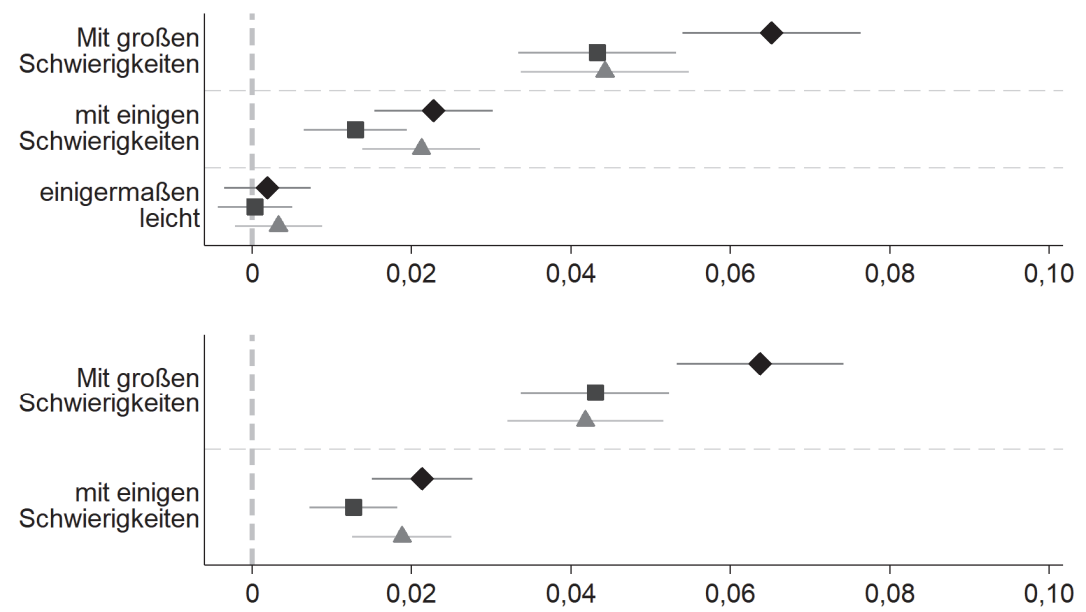

Depression

Einschränkungen in ADL

Schlechte subjektive Gesundheit

Quelle: SHARE Rel. 6.1.1, Wellen 1, 2, 4, 5, 6. Eigene Berechnungen. Darstellung der Koeffizienten und 95\% Konfidenzintervalle. Die kompletten Regressionstabellen befinden sich in Onlineanhang 22.

Änderung in der Wohnsituation von einer mietfreien Wohnung zu einem Mietverhältnis, geht eine Steigerung des Depressionsrisikos um 2,5 Prozentpunkte einher.

Viele Kontrollvariablen zeigen ebenfalls einen signifikanten Zusammenhang mit den Gesundheitsindikatoren auf (siehe Onlineanhang 22). Die folgenden Beschreibungen beziehen sich auf die FE-Modelle. Mit höherem Alter geht schlechtere Gesundheit, gemessen anhand der subjektiven Gesundheit und den körperlichen Einschränkungen, einher. Bei Depression zeigt sich jedoch unter Kontrolle der anderen Faktoren ein negativer Zusammenhang: Mit höherem Alter sinkt das Depressionsrisiko. Der Verlust oder Auszug des Partners hat keinen Einfluss auf die subjektive Gesundheit, jedoch ist damit eine erhöhte Wahrscheinlichkeit von körperlichen Einschränkungen (2,7 Prozentpunkte) und Depressionen (6,1 Prozentpunkte) verbunden. Was den Erwerbsstatus betrifft, so ist der Übergang in die Rente mit besserer Gesundheit verknüpft.Vor allem bei Arbeitslosigkeit und Erwerbsunfähigkeit ist die Wahrscheinlichkeit von schlechter Gesundheit bei allen drei Indikatoren deutlich 
erhöht, bis zu 10,4 Prozentpunkte. ${ }^{16}$ Aber auch Erwerbstätigkeit ist mit einem höherem Depressionsrisiko und Einschränkungen in ADL verknüpft. Der Erhalt von Geschenken ist bei allen drei Gesundheitsmaßen und ein Erbe nur bei Depression mit einer durchschnittlich schlechteren Gesundheit verbunden. Wenig überraschend ist, dass alle Gesundheitsindikatoren, die in den Berechnungen als unabhängige Variablen enthalten sind, recht starke Zusammenhänge mit den abhängigen Variablen aufzeigen. Dies zeigt, dass mentale und physische Gesundheit eng zusammenhängen. Außerdem ist im Vergleich zu Welle 1 in späteren Wellen die Gesundheit der Befragten schlechter - bezüglich Depressionen und Einschränkungen in den ADL mit zunehmender Tendenz im Zeitverlauf.

\subsection{Robustheitsanalysen}

Um sicherzustellen, dass die dargestellten Zusammenhänge nicht von einzelnen methodischen Entscheidungen abhängig sind, sind alternative Berechnungen sinnvoll. Daher werden die Analysen auch für verschiedene alternative Operationalisierungen und andere Variablen durchgeführt (siehe Onlineanhang 23). Verwendet man anstatt der binären Indikatoren die metrischen Werte für die Anzahl der depressiven Symptome oder die Anzahl der Einschränkungen, so bestätigen sich die signifikanten Zusammenhänge mit der finanziellen Situation des Haushaltes. Diesen Berechnungen zufolge geht der Wechsel von keinen Schwierigkeiten zu großen finanziellen Schwierigkeiten durchschnittlich mit einem Anstieg von 0,4 depressiven Symptomen und 0,1 Einschränkungen in Aktivitäten einher. Auch die Verwendung eines anderen Indikators, der körperliche Einschränkungen misst, untermauert die bisherigen Befunde: Finanzielle Schwierigkeiten sind verknüpft mit Einschränkungen in der Mobilität (gemessen sowohl als binäre als auch als metrische Variable). Die Zusammenhänge finden sich ebenfalls, wenn die 5-stufige Skala, mit der die subjektive Gesundheit erhoben wird, als metrische abhängige Variable verwendet wird. Außerdem werden die Ergebnisse von Logit-Fixed-Effects Modellen bestätigt (siehe Onlineanhang 24).

$\mathrm{Ob}$ die anderen Gesundheitsindikatoren kontrolliert werden oder nicht, macht für die Ergebnisse keinen grundlegenden Unterschied (siehe Tabelle 5.2). Sowohl die Koeffizienten der finanziellen Probleme als auch der Schulden sind unter Kontrolle der Gesundheitsindikatoren jedoch etwas kleiner. Die Varianzaufklärung des Gesamtmodells ist ohne die Kontrolle der Gesundheitsvariablen deutlich geringer.

16 In den Berechnungen stellt „Rente“ die Referenzkategorie für die Variablen des Erwerbsstatus dar, siehe z.B. Onlineanhang 22. 
Tabelle 5.2: Fixed-Effects-Modelle mit und ohne Gesundheit als Kontrollvariable

\begin{tabular}{|c|c|c|c|c|c|c|}
\hline & \multicolumn{2}{|c|}{ Depression } & \multicolumn{2}{|c|}{ Einschränkung in ADL } & \multicolumn{2}{|c|}{$\begin{array}{l}\text { Schlechte subjektive } \\
\text { Gesundheit }\end{array}$} \\
\hline & (1) & $(2)$ & (3) & (4) & (5) & (6) \\
\hline $\begin{array}{l}\text { Mit großen } \\
\text { Schwierigkeiten }\end{array}$ & $\begin{array}{l}0,064^{* * *} \\
(0,01)\end{array}$ & $\begin{array}{l}0,075^{* * *} \\
(0,01)\end{array}$ & $\begin{array}{l}0,043^{* * *} \\
(0,00)\end{array}$ & $\begin{array}{l}0,050^{* * *} \\
(0,00)\end{array}$ & $\begin{array}{l}0,042^{* * *} \\
(0,00)\end{array}$ & $\begin{array}{l}0,051^{* \star \star} \\
(0,01)\end{array}$ \\
\hline $\begin{array}{l}\text { Mit einigen } \\
\text { Schwierigkeiten }\end{array}$ & $\begin{array}{l}0,021^{* * *} \\
(0,00)\end{array}$ & $\begin{array}{l}0,026^{* * *} \\
(0,00)\end{array}$ & $\begin{array}{l}0,013^{* * *} \\
(0,00)\end{array}$ & $\begin{array}{l}0,015^{* * *} \\
(0,00)\end{array}$ & $\begin{array}{l}0,019^{* * *} \\
(0,00)\end{array}$ & $\begin{array}{l}0,022^{* \star \star} \\
(0,00)\end{array}$ \\
\hline $\begin{array}{l}\text { Schulden oder } \\
\text { Hypothek }\end{array}$ & $\begin{array}{l}0,029^{* \star *} \\
(0,00)\end{array}$ & $\begin{array}{l}0,034^{* \star *} \\
(0,00)\end{array}$ & $\begin{array}{l}0,021^{* * *} \\
(0,00)\end{array}$ & $\begin{array}{l}0,024^{* * *} \\
(0,00)\end{array}$ & $\begin{array}{l}0,016^{\star \star \star} \\
(0,00)\end{array}$ & $\begin{array}{l}0,020^{\star \star \star} \\
(0,00)\end{array}$ \\
\hline $\begin{array}{l}\text { Einschrän- } \\
\text { kung(en) in } A D L\end{array}$ & $\begin{array}{l}0,109^{\star * *} \\
(0,00)\end{array}$ & & & & & \\
\hline $\begin{array}{l}\text { Schlechte subj. } \\
\text { Gesundheit }\end{array}$ & $\begin{array}{l}0,121^{* * *} \\
(0,00)\end{array}$ & & & & & \\
\hline Depression & & & $\begin{array}{l}0,093^{\star \star \star} \\
(0,00)\end{array}$ & & $\begin{array}{l}0,125^{\star \star \star} \\
(0,00)\end{array}$ & \\
\hline Within- $R^{2}$ & 0,033 & 0,008 & 0,029 & 0,017 & 0,035 & 0,019 \\
\hline Beobachtungen & 189.647 & 189.647 & 189.647 & 189.647 & 189.647 & 189.647 \\
\hline Personen & 67.599 & 67.599 & 67.599 & 67.599 & 67.599 & 67.599 \\
\hline
\end{tabular}

Quelle: SHARE Rel. 6.1.1, Wellen 1, 2, 4, 5, 6. Eigene Berechnungen, Standardfehler in Klammern. Die Modelle enthalten weitere Kontrollvariablen, die vollständige Tabelle kann in Onlineanhang 26 nachgeschlagen werden.

Auch eine alternative Operationalisierung der Schulden hat nur sehr geringe Auswirkungen auf die Berechnungen. Ist man am Nettovermögen interessiert, dann zählen bspw. Kredite, die für die Finanzierung von Wohneigentum aufgenommen wurden, nicht als Schulden, da die ausstehenden Zahlungen mit dem Wert der Immobilie verrechnet werden. Werden Schulden auf Wohneigentum in den Analysen ausgeschlossen, so ist der Koeffizient etwas geringer als unter Berücksichtigung aller Schulden (siehe Onlineanhang 25). Werden Schulden auf Wohneigentum und sonstige Schulden getrennt in die Modelle aufgenommen, so haben beide Variablen positive Koeffizienten, die fast alle signifikant sind. Für die Koeffizienten der finanziellen Probleme hat die Wahl der Operationalisierung der Schulden keinerlei Auswirkungen.

\subsection{Kausalität}

Um dem kausalen Effekt der finanziellen Lage auf die Gesundheit näherzukommen, werden Fixed-Effects-Modelle anhand einer speziellen Teilstich- 
probe berechnet. Diese Stichprobe besteht nur aus den Beobachtungen, bei denen die Befragten angegeben haben, im Ruhestand zu sein. Auf diese Weise kann der Mechanismus, dass der Erwerbsstatus und somit die finanzielle Situation durch den Gesundheitsstatus bestimmt wird, ausgeschlossen werden. Wenn der Zusammenhang zwischen finanzieller Lage und Gesundheit in beide Richtungen kausal verknüpft ist, dann sollte der Zusammenhang unter Ausschluss eines Pfades schwächer werden.

Die Teilstichprobe der Rentner*innen unterscheidet sich von der Gesamtstichprobe nur in wenigen Variablen (siehe Onlineanhang 28). Das Durchschnittsalter ist bei den Rentnern über 5 Jahre höher als in der gesamten Stichprobe. In der finanziellen Situation der Haushalte gibt es keine Unterschiede zwischen den Stichproben. Beim Anteil der verschuldeten Haushalte ist hingegen ein deutlicher Unterschied vorhanden. So ist in der Stichprobe der Rentner*innen der Anteil der Personen, die in Haushalten mit Schulden leben, um fast 10 Prozentpunkte geringer. Was die Gesundheit betrifft, so sind Einschränkungen in Aktivitäten sowie schlechte subjektive Gesundheit unter Rentnern und Rentnerinnen häufiger als in der gesamten Stichprobe. Bei der Prävalenz von Depression gibt es keinen Unterschied.

Wird in dieser Teilstichprobe ein Zusammenhang von finanzieller Situation und Gesundheit gefunden, so kann ausgeschlossen werden, dass dieser Zusammenhang zustande kommt, weil Personen aufgrund schlechter Gesundheit ihren Arbeitsplatz verloren haben oder (früher) in Ruhestand gegangen sind. Umgekehrte Kausalität wird somit teilweise ausgeschlossen. Ein anderer bedeutender Pfad umgekehrter Kausalität, die individuellen Gesundheitsausgaben, kann hier aufgrund fehlender Informationen nicht berücksichtigt werden.

In Abbildung 5.14 ist der Vergleich von FE-Modellen der beiden Stichproben dargestellt. Für Depression und subjektive Gesundheit bestätigt sich die Vermutung, dass die Koeffizienten in der Teilstichprobe der Personen in $\mathrm{Ru}-$ hestand etwas geringer ausfallen als in der gesamten Stichprobe. Für Einschränkungen in Aktivitäten des täglichen Lebens zeigt sich hingegen ein stärkerer Zusammenhang mit der finanziellen Situation sobald nur Rentner und Rentnerinnen analysiert werden. Bei allen abhängigen Variablen sind die Punktschätzer, die aus den unterschiedlichen Stichproben resultieren, jedoch nicht signifikant unterschiedlich. Die deutlich breiteren Konfidenzintervalle in dem Modell, in dem ausschließlich Personen in Ruhestand enthalten sind, resultieren aus der geringeren Fallzahl. 
Abbildung 5.14: Fixed-Effects Modelle. Vergleich der Gesamtstichprobe mit der Teilstichprobe „Rentner“
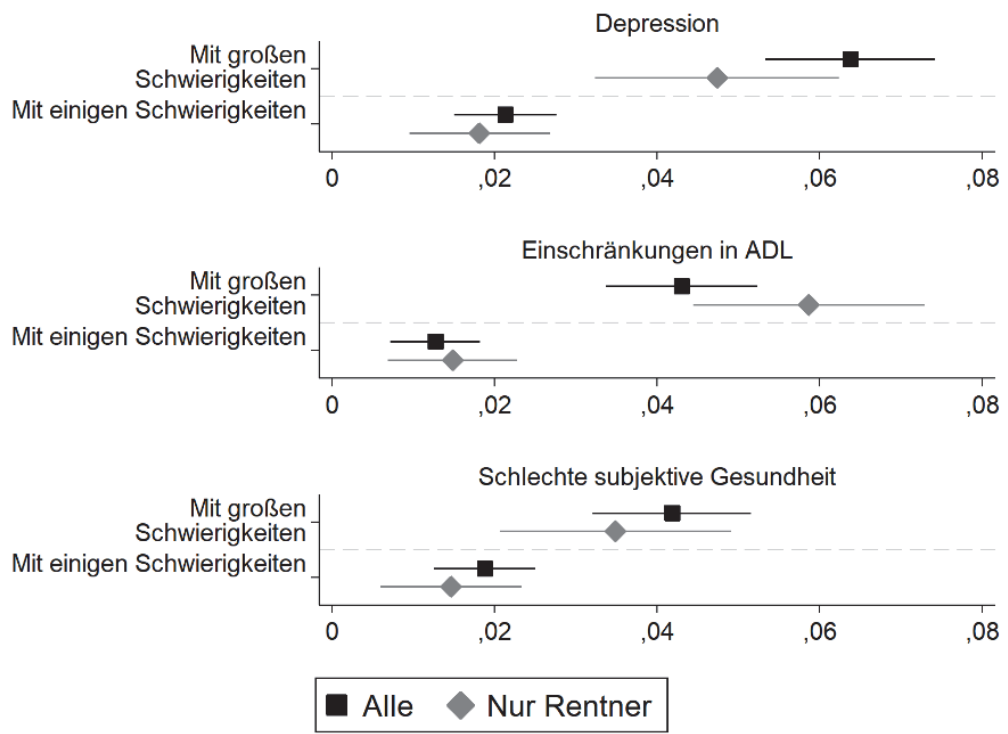

Quelle: SHARE Rel. 6.1.1, Wellen 1, 2, 4, 5, 6. Eigene Berechnungen. Darstellung der Koeffizienten und 95\%-Konfidenzintervalle. Die kompletten Regressionstabellen befinden sich in Onlineanhang 27.

\subsection{Diskussion}

Die dargestellten Ergebnisse bestätigen die bekannten Muster im Zusammenhang von finanziellen Schwierigkeiten und Gesundheit auch für die ältere Bevölkerung in Europa. Dass mit einer schlechten finanziellen Lage des Haushaltes ein höheres Risiko von schlechter Gesundheit einhergeht, zeigen sowohl die bivariaten als auch die multivariaten Analysen. Der Vergleich verschiedener Analysemethoden verdeutlicht, dass Querschnittanalysen bei dieser Fragestellung nicht geeignet sind, um einen kausalen Effekt zu identifizieren. Unter der Kontrolle der individuellen zeitkonstanten Eigenschaften der Befragten zeigen sich deutlich kleinere Regressionskoeffizienten als unter Verwendung von Querschnittsmethoden. Dies ist konsistent mit bisherigen Befunden (z.B. Jones/Wildman 2008; Frijters/Ulker 2008; Imlach Gunasekara/Carter/Blakely 2011). 
Die Fixed-Effects-Modelle untersuchen wie sich die Veränderung in der finanziellen Lage auf die Veränderung der Gesundheit auswirkt. Insgesamt erweisen sich die Ergebnisse als sehr robust. Sie bleiben weitgehend unbeeinflusst von alternativen Indikatoren und Operationalisierungen der abhängigen sowie unabhängigen Variablen. Den Analysen zufolge erhöhen einige finanzielle Schwierigkeiten die Wahrscheinlichkeit von schlechter Gesundheit um ca. einen Prozentpunkt bei Einschränkungen in ADL und um ca. 2 Prozentpunkte bei Depression sowie subjektiver Gesundheit. Der Effekt von großen finanziellen Schwierigkeiten ist mehr als doppelt so stark, über 4 Prozentpunkte bei Einschränkungen der ADL und subjektiver Gesundheit sowie mehr als 6 Prozentpunkte bei Depression. Um diese Zahlen besser einordnen zu können ist ein Blick auf die Kontrollvariablen angebracht. Die Wahrscheinlichkeit depressiv zu sein wird von dem Auszug oder Tod des Partners etwa gleich stark beeinflusst wie durch eine Verschlechterung der finanziellen Lage, jeweils um etwa 6 Prozentpunkte. Für die Einschränkungen in den ADL hat das Zusammenleben mit dem Partner einen geringeren Einfluss, für die subjektive Gesundheit kann kein signifikanter Zusammenhang festgestellt werden. Dieser Vergleich verdeutlicht, dass Veränderungen in der finanziellen Lage trotz der relativ kleinen Koeffizienten für die Gesundheit im Alter von Bedeutung sind.

Wie die Analysen zeigen, können finanzielle Notlagen die physische Gesundheit beeinflussen. Theoretisch ist es plausibel, dass Folgen für die physische Gesundheit mit einer gewissen Verzögerung eintreten während sich die Auswirkungen auf die mentale Gesundheit, z.B. in Form von Sorgen und Ängsten, sofort bemerkbar machen können. Ist eine Veränderung in der finanziellen Situation schon vorher bekannt, z.B. eine Kündigung des Arbeitsvertrages oder der Eintritt in den Ruhestand, so kann dies schon im Vorfeld der eigentlichen Veränderung Auswirkungen auf die mentale Verfassung haben. Daher ist es plausibel, dass die Zusammenhänge mit der finanziellen Situation für die Indikatoren physischer Gesundheit geringer ausfallen als für den Indikator der mentalen Gesundheit.

In der bivariaten Analyse zeigt sich zunächst eine unerwartete Korrelation von Schulden und Gesundheit. Schulden gehen demnach mit etwas besserer Gesundheit einher. Eine mögliche Erklärung könnte sein, dass sich eher die höheren Statusgruppen (die über bessere Gesundheit verfügen) einen größeren Kredit „leisten“ können, da sie über ausreichend (zukünftiges) Einkommen verfügen um langfristig planen zu können (vgl. Drentea/Reynolds 2015). Anhand der vorliegenden Daten kann dies insofern bestätigt werden, dass in der Stichprobe der Anteil der Personen, die von Schulden berichten, mit höherem Bildungsgrad ansteigt (siehe Onlineanhang 29). Der bivariate Zusammenhang wird jedoch in den multivariaten Analysen nicht bestätigt. Unter Kontrolle anderer Einflussfaktoren zeigt sich der erwartete Effekt, dass eine neue Verschuldung mit einer höheren bzw. die vollständige Tilgung von Schulden mit einer 
geringeren Wahrscheinlichkeit von schlechterer Gesundheit verknüpft ist. Dieser Zusammenhang wurde bislang vor allem für Depressionen aufgezeigt (vgl. Hiilamo/Grundy 2018; Richardson/Elliott/Roberts 2013). Das Ergebnis, dass Schulden weniger stark mit der Gesundheit korrelieren als die subjektiv empfundenen finanziellen Probleme, untermauert zudem bisherige Befunde, dass objektive Indikatoren weniger stark mit Gesundheit korrelieren als subjektive Maße (Bridges/Disney 2010).

Dass die physische Gesundheit für die mentale Gesundheit von Bedeutung ist, lässt sich einfach nachvollziehen, nicht nur anhand von schweren Erkrankungen, die die Lebensqualität einschränken oder mit denen eine geringe Überlebenswahrscheinlichkeit einhergeht. Dass psychische Probleme für physische Krankheiten eine Rolle spielen ist hingegen weniger intuitiv, aber durch eine Vielzahl an Studien bestätigt (Bertoni et al. 2018; Penninx 1998; Hays et al. 1997). Auch in den hier durchgeführten Analysen wird dies deutlich. In allen Modellen zur Erklärung von Einschränkungen in den ADL und subjektiver Gesundheit besteht eine starke Korrelation mit Depression. Die beiden abhängigen Variablen sind mit Depression stärker verknüpft als mit finanziellen Problemen. In den Analysen zur mentalen Gesundheit werden die beiden Maße physischer Gesundheit kontrolliert, und umgekehrt, um bestmöglich die direkten Effekte zu identifizieren. In zusätzlichen Analysen werden diese Berechnungen mit Modellen verglichen, in denen die Gesundheitsindikatoren nicht kontrolliert werden. Sowohl die Koeffizienten der finanziellen Probleme als auch die Koeffizienten der Schulden sind unter Kontrolle der Gesundheit etwas kleiner. Das deutet darauf hin, dass ein kleiner Teil des Zusammenhangs über die zusätzlich kontrollierten Gesundheitsindikatoren vermittelt ist. Bei allen drei untersuchten Gesundheitsmaßen ist jedoch der direkte Effekt deutlich stärker.

Die Analysen, die ausschließlich mit Personen, die sich im Ruhestand befinden, durchgeführt werden, dienen der Annäherung an die wahren kausalen Effekte. Ein Pfad für mögliche umgekehrte Kausalität - schlechte Gesundheit führt zu Verlust der Arbeit, Reduzierung der Arbeitszeit oder frühzeitigem Renteneintritt - kann auf diese Weise ausgeschlossen werden. Die Koeffizienten für die Variablen der finanziellen Probleme unterscheiden sich etwas zwischen der Teilstichprobe der Rentner und der gesamten Stichprobe. Diese Unterschiede sind zwar nicht signifikant und sollten daher mit großer Vorsicht behandelt werden, dennoch geben sie Hinweise auf mögliche Mechanismen. Dass der Zusammenhang zwischen finanziellen Problemen und Depression bzw. subjektiver Gesundheit eher geringer wird, sobald ein möglicher Pfad der umgekehrten Kausalität ausgeschlossen wird, entspricht den Erwartungen. Dies deutet darauf hin, dass der vermutete Pfad der umgekehrten Kausalität zwar eine gewisse Rolle spielt, aber nicht die Hauptursache für die Korrelation darstellt. Auch eine aktuelle Studie unterstützt dieses Ergebnis, wonach im höheren Alter (beim Übergang vom Erwerbsleben in die Rente) hauptsächlich 
der kausale Effekt (von finanziellen Problemen auf Gesundheit) wirkt (Hoffmann/Kröger/Pakpahan 2018a). Zudem entspricht es dem Ergebnis, dass der sozioökonomische Status gemessen anhand von finanziellen Maßen eher die kausale Hypothese stützt, im Gegensatz zu Indikatoren, die mit der beruflichen Position verknüpft sind (Kröger/Pakpahan/Hoffmann 2015). Ein anderes Bild zeigt sich für die Korrelation von finanziellen Problemen mit den Einschränkungen in ADL, die in der Stichprobe der Rentner*innen stärker wird. Dies könnte mit dem höheren Alter der Befragten in dieser Teilstichprobe zusammenhängen. Dass für die Unterschiede zwischen den Stichproben das Alter eine Rolle spielt wird dadurch gestützt, dass dieselben Muster zu sehen sind, wenn die gesamte Stichprobe in drei Altersgruppen geteilt wird (siehe Onlineanhang 30). Die Anfälligkeit für eine Depression bei Krisen könnte mit zunehmendem Alter aufgrund der größeren Lebenserfahrung im Umgang mit schwierigen Situationen geringer werden (Mirowsky/Ross 2001; Stoppe 2008; Westerhof/Tulle/Emmanuelle 2008; Keller/Leventhal/Larson 1989). Im Gegensatz dazu erhöht sich mit höherem Alter die Anfälligkeit für körperliche Einschränkungen (Slaets 2006; Topinková 2008) und finanzielle Notlagen können somit eher auf diesen Aspekt der Gesundheit wirken. In diesem Zusammenhang wäre es wünschenswert die individuellen Gesundheitsausgaben als weiteren möglichen Pfad der umgekehrten Kausalität in den Analysen zu berücksichtigen. Mit den vorliegenden Daten ist dies jedoch (im Längsschnitt) nicht möglich.

Um die Diskussion der Analysen zu vervollständigen, werden nun die Ergebnisse der Kontrollvariablen beschrieben. Die Effekte des Zusammenlebens mit einem Partner im Haushalt auf die Gesundheit wurden bereits oben erwähnt. Diese stimmen überein mit dem bekannten protektiven Effekt einer Partnerschaft (Carr/Springer 2010) bzw. den negativen gesundheitlichen Folgen bei Verlust des Partners durch Trennung oder Tod (Schaan 2013b; Wilcox et al. 2003). In den deskriptiven Analysen wird zudem festgestellt, dass mit höherem Alter eine Verschlechterung der Gesundheit einhergeht, besonders deutlich ist dies für die Einschränkungen der Aktivitäten zu erkennen. In den Berechnungen mit OLS, POLS und RE kann dieser Zusammenhang zunächst bestätigt werden. Allerdings dreht sich bei der Analyse der Depressionswahrscheinlichkeit das Vorzeichen in dem FE-Modell um. Ein höheres Alter geht demnach bei den über 50-Jährigen mit einer geringeren Depressionswahrscheinlichkeit einher. Dies wurde bereits an anderer Stelle in multivariaten Analysen berichtet (Heflin/Iceland 2009; Carter et al. 2009). Bei der Betrachtung des Zusammenhangs des Erwerbsstatus mit der Gesundheit fällt auf, dass die Rentner*innen über eine bessere Gesundheit verfügen als erwerbstätige, arbeitslose oder erwerbsunfähige Personen. Die Unterschiede zwischen dem Erwerbsstatus zeigen sich auch in den Längsschnittanalysen mit einer anderen Interpretation. Demnach ist der Wechsel in den Ruhestand mit einer Erhöhung der Wahrscheinlichkeit für eine gute Gesundheit verknüpft. Dies entspricht 
den Ergebnissen aus der Forschung zu den gesundheitlichen Folgen des Renteneintritts (Hanemann 2018; Insler 2014; Coe/Zamarro 2011). Begründet wird dies mit der Entlastung von anstrengender Tätigkeit und zunehmender Freizeit, in der man sich auch gesundheitsfördernden Aktivitäten widmen kann (Celidoni/Rebba 2017; Insler 2014). Dementsprechend wird ein positiver Effekt auf die Gesundheit vor allem bei schlechten Arbeitsbedingungen (Hanemann 2018) und körperlich anstrengender Arbeit gefunden (Mazzonna/Peracchi 2017).

Insgesamt tragen diese Ergebnisse zur weiteren detaillierten Beschreibung des Zusammenhangs von finanzieller Lage und Gesundheit bei. Eine Besonderheit ist hierbei, dass kein binärer Indikator für Armut, sondern eine mehrstufige Skala, verwendet wird, mit der Unterschiede zwischen einigen und großen finanziellen Schwierigkeiten sichtbar werden. Die Fixed-Effects-Berechnungen zur Erklärung der Veränderungen von körperlichen Einschränkungen sind von besonderer Bedeutung, da bislang keine vergleichbare Studie existiert. In Anbetracht der großen Relevanz von Mobilität und Selbstständigkeit für die Lebenszufriedenheit von älteren Menschen (Netuveli et al. 2006; Andersen et al. 2004), ist es eine wichtige Erkenntnis, dass finanzielle Faktoren mit der Entwicklung von körperlichen Einschränkungen in Zusammenhang stehen. Hierbei ist jedoch zu bedenken, dass die Gesundheitsausgaben der Befragten, bzw. ihres Haushaltes, nicht in den Analysen berücksichtigt werden konnten. Wenn Einschränkungen bei Aktivitäten die Anschaffung von Hilfsmitteln oder die Inanspruchnahme von Dienstleistungen (z.B. von Pflegekräften oder Haushaltshilfen) erfordern und somit zu finanziellen Schwierigkeiten führen, liegt umgekehrte Kausalität vor. Es kann hier nicht festgestellt werden, wie stark die körperlichen Einschränkungen auf die finanzielle Lage wirken und wie groß der entgegengesetzte Effekt ist. Daher sollten diese Ergebnisse mit Vorsicht interpretiert werden. Umgekehrte Kausalität kann auch bei den Analysen von Depression und subjektiver Gesundheit nicht ausgeschlossen werden, jedoch sind die Ausgaben bei anderen Krankheiten oftmals geringer als für die notwendigen Hilfsmittel und Dienstleistungen bei körperlichen Einschränkungen, bzw. werden psychische Krankheiten häufig überhaupt nicht therapiert (Stoppe 2008).

Aus den besprochenen Ergebnissen können praktische bzw. politische Maßnahmen für die Bekämpfung von gesundheitlicher Ungleichheit abgeleitet werden. Die zwar schon bekannte, aber dennoch wichtigste Botschaft ist, dass Armut krank macht. Wie die Analysen zeigen, haben nicht nur große, sondern auch geringe Sorgen negative Effekte für die Gesundheit. Das gesellschaftliche Ziel sollte daher sein, Armut und Armutsgefährdung zu bekämpfen (vgl. BMAS 2017). Eine Möglichkeit die vollständige Eliminierung von Armut zu erreichen, könnte bspw. das umstrittene Konzept des bedingungslosen Grundeinkommens sein (vgl. Spannagel 2015). Da gezeigt wurde, dass Verschuldung 
einen eigenständigen negativen Effekt auf die Gesundheit aufweist, sind verschuldete Personen und Haushalte eine klar definierte Zielgruppe für mögliche Beratungs- und Interventionsmaßnahmen (vgl. Hiilamo/Grundy 2018). 


\section{Analysen: Verbesserung und Verschlechterung der finanziellen Situation}

Nachdem das vorherige Kapitel gezeigt hat, dass finanzielle Probleme auch im Längsschnitt mit einer Verschlechterung von Gesundheit einhergehen, soll in diesem Kapitel die Richtung der Änderungen in der finanziellen Situation betrachtet werden. In einem FE-Modell wird die Gesundheit von Personen zu verschiedenen Zeitpunkten bzw. unterschiedlichen finanziellen Situationen verglichen. Die Wahl der Referenzkategorie (keine finanziellen Probleme) und die gängige Vorstellung, dass Armut krank macht, verleitet zu der Interpretation der FE-Berechnungen, dass finanzielle Probleme krank machen. Theoretisch könnte jedoch ein signifikanter Koeffizient in den Analysen zustande kommen, der ausschließlich darauf beruht, dass die Verbesserung der finanziellen Lage gesünder macht, ohne dass ein gesundheitsschädlicher Effekt der Verschlechterung der finanziellen Lage existiert. Das FE-Modell liefert lediglich einen Mittelwert aus beiden möglichen Effekten. In welcher Reihenfolge die verschiedenen Zustände vorkommen ist in diesen Analysen nicht von Bedeutung.

Bei Analysen der Gesundheit ist es plausibel, dass Veränderungen in der finanziellen Lage in beide Richtungen einen Einfluss haben. Es stellt sich die Frage, wie stark die einzelnen Effekte von Verbesserung und Verschlechterung der finanziellen Situation ausfallen. Für die Beantwortung dieser Frage wird auf ein First-Difference-Modell (FD-Modell) zurückgegriffen (siehe Kapitel 4.6.4). Im Gegensatz zum FE-Modell, das den gesamten Untersuchungszeitraum nutzt, um die Koeffizienten zu berechnen, werden im FD-Modell nur zwei aufeinanderfolgende Zeitpunkte verglichen. Dadurch eignet es sich für die Identifikation von unmittelbaren Effekten. Die Stichprobe, die analysiert werden kann, unterscheidet sich von der Stichprobe der FE-Berechnungen. Von jeder Person fällt die jeweils erste Beobachtung aus der Analyse, da hierfür kein Vergleich mit einer vorherigen Beobachtung möglich ist. Zudem werden nur aufeinanderfolgende Beobachtungen einer Person berücksichtigt, die maximal zwei Untersuchungswellen auseinander liegen (siehe Kapitel 4.6.4). Abgesehen von der - durch die Methode bedingten - unterschiedlichen Verteilung über die Untersuchungswellen, ist die Stichprobe, die für die FD-Berechnung verwendet wird, der Stichprobe der FE-Analysen sehr ähnlich (siehe Onlineanhang 31). Lediglich ein etwas erhöhter Anteil von Personen im Ruhestand kann festgestellt werden. 


\subsection{First-Difference-Analysen}

In diesem Abschnitt wird beschrieben wie sich eine Veränderung in der finanziellen Lage auf die Wahrscheinlichkeit von Depression, Einschränkungen in ADL und schlechter subjektiver Gesundheit auswirkt. Wie in Kapitel 4.6.4 beschrieben, werden in das FD-Modell alle möglichen Pfade in der finanziellen Situation zwischen zwei Zeitpunkten als Dummyvariablen aufgenommen. Die Effekte dieser Pfade sind in Abbildung 6.1 dargestellt. Da 55 Prozent der Beobachtungen zu beiden Zeitpunkten keine finanziellen Probleme hatten, dient dies als Referenzkategorie. ${ }^{17}$

Abbildung 6.1: First-Difference-Modell - Effekte von Veränderung der finanziellen Schwierigkeiten auf Depression

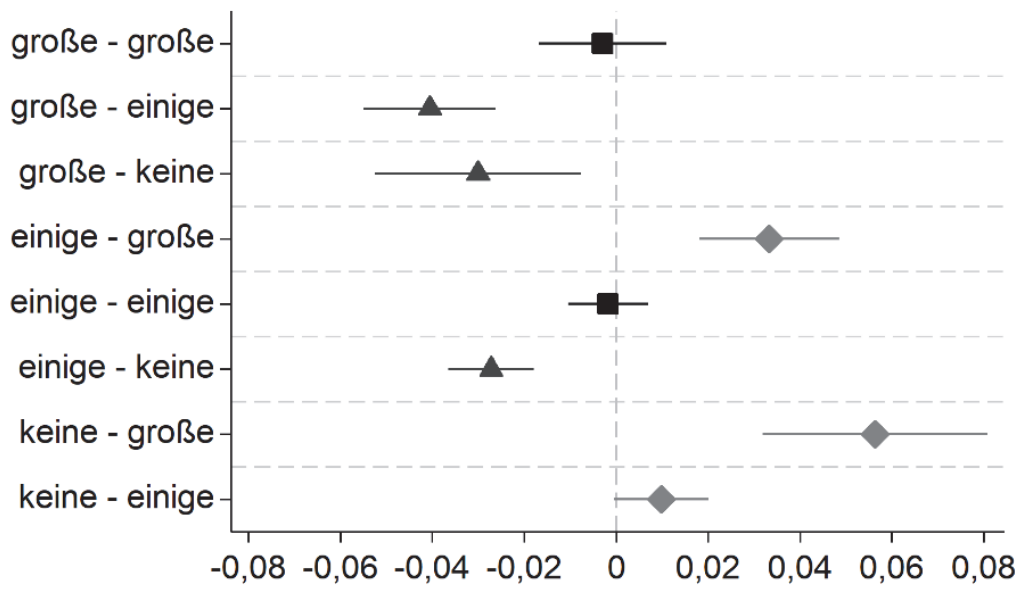

- Verbesserung der finanziellen Situation

- keine Veränderung

Verschlechterung der finanziellen Situation

Quelle: SHARE Rel. 6.1.1, Wellen 1, 2, 4, 5, 6. Eigene Berechnungen. Darstellung der Koeffizienten und $95 \%$-Konfidenzintervalle. Die komplette Regressionstabelle befindet sich in Onlineanhang 32.

17 Die Kategorien „einigermaßen leicht“ und „leicht“ werden hier als „keine finanziellen Schwierigkeiten" zusammengefasst. In Kapitel 5.2 wurde dargestellt, dass in den FE-Analysen zwischen diesen beiden Kategorien kein Unterschied im Zusammenhang mit Gesundheit festgestellt werden kann. 
Konstante finanzielle Schwierigkeiten des Haushaltes haben keine Auswirkungen auf das Depressionsrisiko. Eine Verschlechterung der finanziellen Situation geht tendenziell mit einer höheren Depressionswahrscheinlichkeit einher. Die Verschlechterung von keinen zu einigen Schwierigkeiten ist, gemessen anhand einer Irrtumswahrscheinlichkeit von 5 Prozent, knapp nicht signifikant. Eine Verbesserung der finanziellen Situation ist immer mit einer signifikanten Reduzierung der Depressionswahrscheinlichkeit verknüpft. Bei Haushalten, die große finanzielle Schwierigkeiten hatten und diese überwinden konnten, ist kein Unterschied zu erkennen zwischen einem Wechsel zu einigen Schwierigkeiten und einer Verbesserung zu keinen finanziellen Problemen. Die beiden Koeffizienten sind nicht signifikant verschieden, sie unterscheiden sich jedoch signifikant von dem Koeffizienten von großen Schwierigkeiten zu beiden Zeitpunkten ( $\mathrm{p}<0.001$ bzw. $\mathrm{p}<0,05)$. Die Effektstärken bei Veränderung der finanziellen Situation in dem FD-Modell sind den Koeffizienten der FE-Modelle sehr ähnlich. Ausgehend von einigen finanziellen Problemen erhöht sich die Wahrscheinlichkeit einer Depression durch eine Verschlechterung der finanziellen Lage um ca. 3 Prozentpunkte. Eine Verbesserung dieser finanziellen Situation führt zu einem um ca. 3 Prozentpunkte geringeren Depressionsrisiko.

Ein sehr ähnliches Ergebnis liefert die FD-Analyse für die Einschränkungen in den ADL, die in Abbildung 6.2 dargestellt ist. Verschlechterungen in der finanziellen Situation gehen mit einer höheren Wahrscheinlichkeit von körperlichen Einschränkungen einher, während Verbesserungen der finanziellen Lage die Wahrscheinlichkeit von Einschränkungen verringern. Auch die Effekte von Verbesserung und Verschlechterung bei einigen Schwierigkeiten zum ersten Zeitpunkt sind signifikant verschieden von dem Effekt von einigen Schwierigkeiten zu beiden Zeitpunkten. Wie auch im Modell zur Erklärung von Depression, ist hier das Überwinden von großen finanziellen Schwierigkeiten mit besserer Gesundheit verknüpft, wobei es keinen Unterschied macht, wie stark die Verbesserung der finanziellen Lage ausfällt. Das Auftreten von finanziellen Problemen ist hingegen umso schädlicher für die Gesundheit, je größer die Verschlechterung der finanziellen Lage ausfällt. Ist eine Person zu beiden Zeitpunkten mit einigen finanziellen Problemen konfrontiert, so ist dies, im Vergleich zu der Referenzkategorie keine finanziellen Probleme, mit einer Erhöhung der Wahrscheinlichkeit von Einschränkungen in Aktivitäten verbunden. Auch der Koeffizient von konstanten großen finanziellen Problemen deutet auf eine Verschlechterung der Gesundheit hin, allerdings erweist er sich bei einer Irrtumswahrscheinlichkeit von 5 Prozent nicht als signifikant verschieden von der Referenzkategorie $(\mathrm{p}=0,11)$. 
Abbildung 6.2: First-Difference-Modell - Effekte von Veränderung der finanziellen Schwierigkeiten auf Einschränkungen in ADL

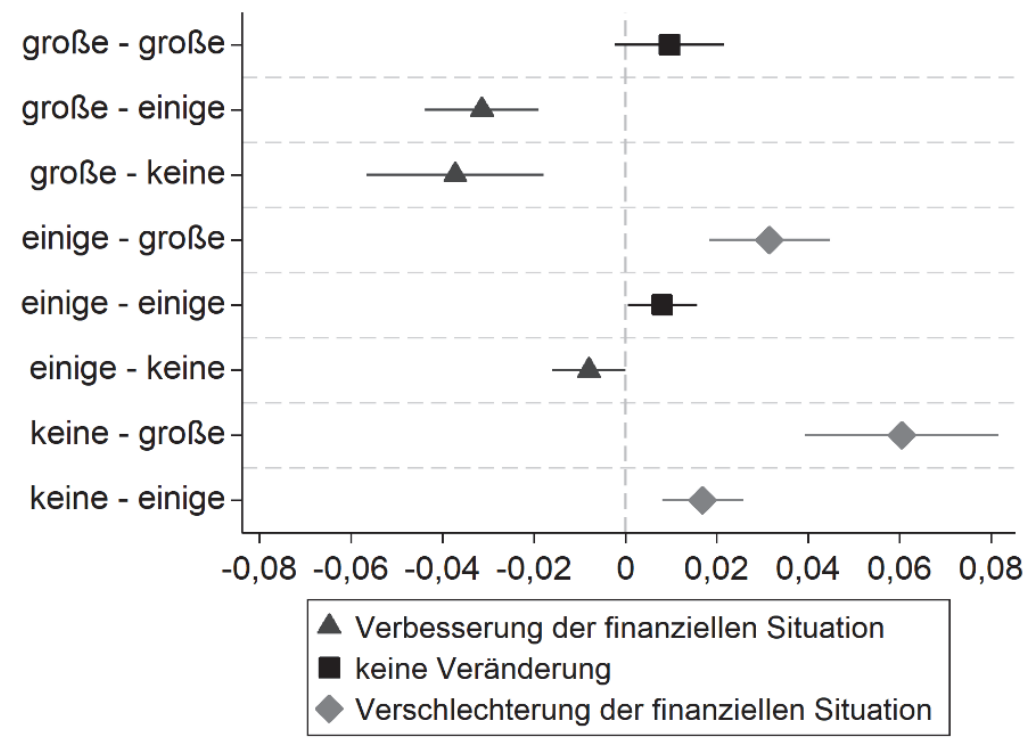

Quelle: SHARE Rel. 6.1.1, Wellen 1, 2, 4, 5, 6. Eigene Berechnungen. Darstellung der Koeffizienten und 95\%-Konfidenzintervalle. Die komplette Regressionstabelle befindet sich in Onlineanhang 32.

Wendet man das FD-Modell auf die subjektive Gesundheit an, so weisen die Koeffizienten zwar das erwartete Vorzeichen auf, das Ergebnis ist jedoch weniger deutlich als bei den beiden zuvor genannten Indikatoren (siehe Abbildung 6.3). Signifikante Effekte zeigen sich nur für den Wechsel von keinen zu einigen Schwierigkeiten, von einigen zu großen Schwierigkeiten sowie für das Überwinden von großen finanziellen Problemen. Allerdings ist der Koeffizient für den Wechsel von großen zu einigen Schwierigkeiten nicht signifikant verschieden von dem Koeffizienten für konstant große Schwierigkeiten.

Um die Hypothese der Verlustaversion zu überprüfen, werden die Koeffizienten von entgegengesetzten Pfaden miteinander verglichen. Es wird getestet, ob der negative Effekt einer Verschlechterung (bspw. von keinen zu einigen Problemen), dem Ausmaß des positiven Effektes bei Verbesserung (von einigen zu keinen Problemen) entspricht oder ob einer der beiden Pfade gröBere Auswirkungen auf die Gesundheit hat als der andere. Da einer der Koeffizienten positiv und der andere negativ ist, wird der Test mit $\beta_{1}=-\beta_{2}$ (anstatt mit $\beta_{1}=\beta_{2}$ ) durchgeführt. Dass die Verbesserung eine größere Auswirkung 
Abbildung 6.3: First-Difference-Modell - Effekte von Veränderung der finanziellen Schwierigkeiten auf schlechte subjektive Gesundheit

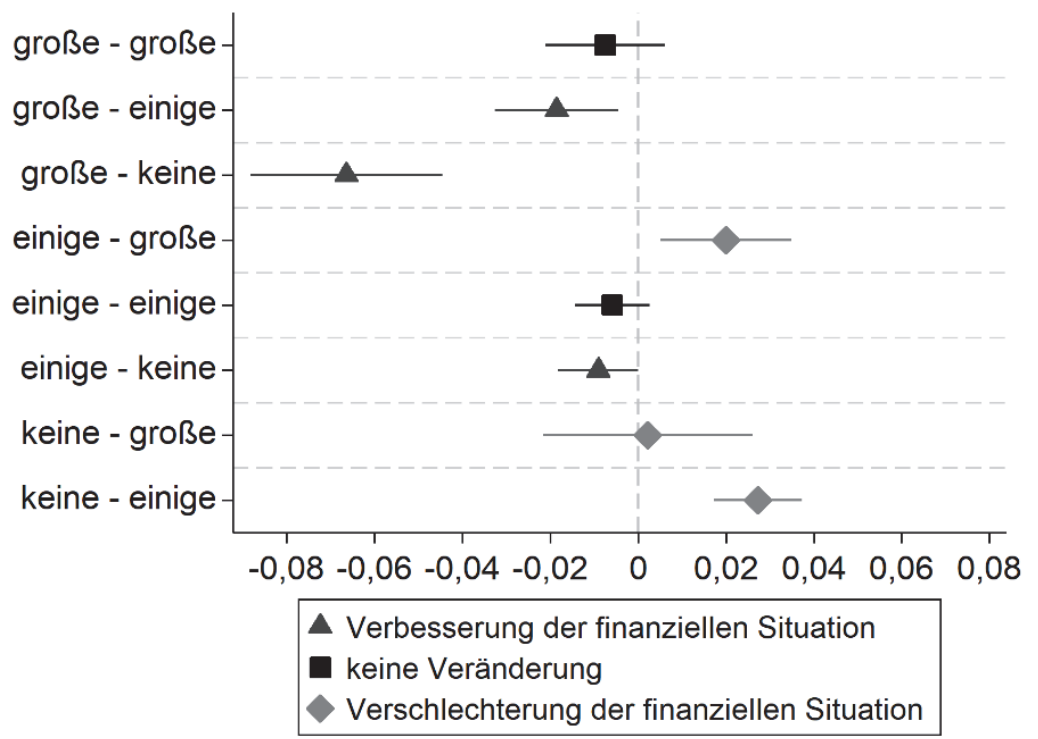

Quelle: SHARE Rel. 6.1.1, Wellen 1, 2, 4, 5, 6. Eigene Berechnungen. Darstellung der Koeffizienten und 95\%-Konfidenzintervalle. Die komplette Regressionstabelle befindet sich in Onlineanhang 32.

auf die Gesundheit hat als eine Verschlechterung, zeigt sich für Depressionbeim Wechsel zwischen keinen und einigen Problemen sowie für die subjektive Gesundheit beim Wechsel zwischen keinen und großen Probleme. Ein stärkerer Effekt bei Verschlechterung findet sich nur für subjektive Gesundheit beim Wechsel zwischen keinen und einigen Problemen. Für alle anderen Pfade kann kein signifikanter Unterschied im Betrag der Effektstärken bei Verbesserung und Verschlechterung der finanziellen Situation festgestellt werden.

Auch für den zweiten Indikator der finanziellen Lage, Schulden, werden Berechnungen nach derselben Methode durchgeführt. Die Ergebnisse sind in Abbildung 6.4 dargestellt. Als Referenz dient auch hier der häufigste Fall: keine Schulden zu beiden Beobachtungszeitpunkten. Bei allen Gesundheitsindikatoren geht eine Verschuldung zu beiden Zeitpunkten, wie auch die vollständige Tilgung von Schulden mit einer geringeren Wahrscheinlichkeit schlechter Gesundheit einher. Eine neue Verschuldung ist hingegen nur bezüglich Depressionen mit negativen gesundheitlichen Folgen verknüpft. Beim Vergleich der beiden Pfade bei Schulden zum ersten Zeitpunkt ist folgendes 
zu beobachten: Für Depressionen hat die Tilgung von Schulden auch im Vergleich zu dauerhaften Schulden positive Auswirkungen auf die Gesundheit. Für Einschränkungen in ADL und die subjektive Gesundheit sind die Koeffizienten jedoch nicht signifikant unterschiedlich (ADL $p=0,10$, subjektive Gesundheit $\mathrm{p}=0,06$ ).

Vergleicht man die Effektstärken von neuer Verschuldung und vollständiger Tilgung der Schulden, so zeigt sich nur für Einschränkungen in ADL, dass der Betrag von positivem und negativem Effekt unterschiedlich groß ist. Wie bereits beschrieben, existiert für Einschränkungen in ADL ein gesundheitsförderlicher Effekt bei Tilgung der Schulden, während eine neue Verschuldung keinen Einfluss auf die Einschränkungen in den ADL hat. Bei Depression und subjektiver Gesundheit sind die Beträge der positiven und negativen Effekte ungefähr gleich groß.

Abbildung 6.4: First-Difference-Modell - Effekte von Veränderung der finanziellen Schwierigkeiten auf Schulden
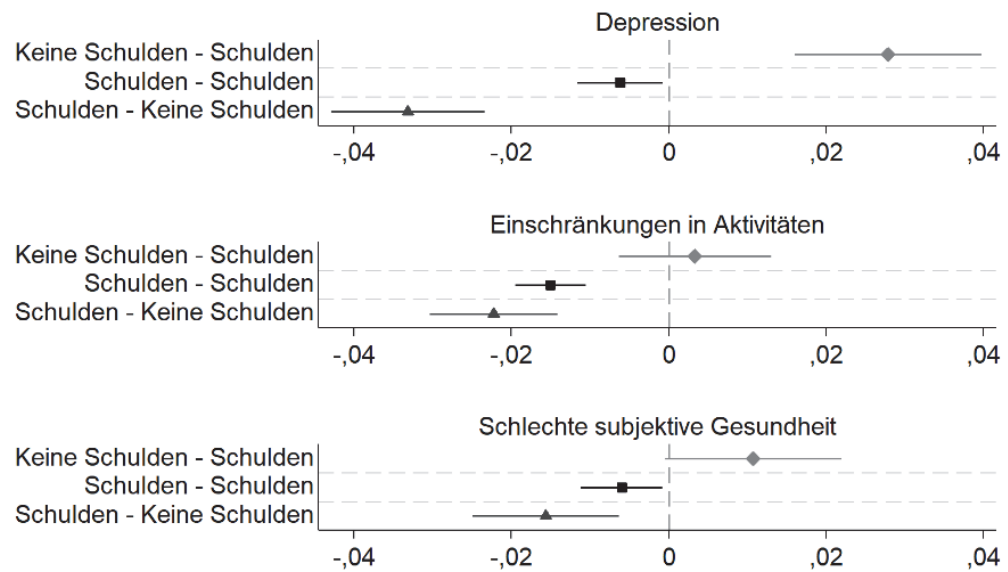

$$
\begin{aligned}
& \text { Verschlechterung der finanziellen Situation } \\
& \text { - keine Veränderung } \\
& \text { - Verbesserung der finanziellen Situation }
\end{aligned}
$$

Quelle: SHARE Rel. 6.1.1, Wellen 1, 2, 4, 5, 6. Eigene Berechnungen. Darstellung der Koeffizienten und 95\%-Konfidenzintervalle. Die komplette Regressionstabelle befindet sich in Onlineanhang 33.

Für die FD-Analysen werden Beobachtungen berücksichtigt, die bis zu zwei Beobachtungswellen auseinanderliegen. Alternative Berechnungen mit einer Beschränkung auf Fälle, die mit nur einer Welle Abstand vorliegen, bestätigen 
die Ergebnisse (siehe Onlineanhang 34). Diese methodische Entscheidung hat demnach keine substanziellen Auswirkungen auf die Ergebnisse.

\subsection{Diskussion}

Die Ergebnisse der FD-Modelle zeigen, dass eine Verbesserung der finanziellen Situation mit einer geringeren Wahrscheinlichkeit einer schlechten Gesundheit einhergeht. Eine Verschlechterung der finanziellen Lage ist hingegen mit einer schlechteren Gesundheit verbunden. Diese Ergebnisse stimmen mit bisherigen Studien überein, wobei der gesundheitsschädliche Effekt bislang in allen Untersuchungen bestätigt werden konnte, der positive Effekt auf die Gesundheit jedoch nicht immer vorzufinden war (vgl. Kapitel 3.2). Diese Zusammenhänge sind für Depressionen und Einschränkungen in ADL stark ausgeprägt, für die subjektive Gesundheit zeigt sich dieses Muster weniger deutlich.

Der Großteil der bisherigen Forschung zu den Auswirkungen von Veränderungen der finanziellen Lage konzentriert sich auf die mentale Gesundheit. Dies hängt vermutlich auch damit zusammen, dass Auswirkungen auf die psychische Gesundheit unmittelbarer, ohne (längere) Latenzzeit, zu erwarten sind. Für Depression wurden die Effekte einer Verbesserung und Verschlechterung der finanziellen Situation bereits in Längsschnittstudien nachgewiesen (Lorant et al. 2007; McKenzie et al. 2014). Für physische Gesundheit sind bislang nur Studien vorhanden, die in diesem Zusammenhang die subjektive Gesundheit untersuchen. Sie weisen darauf hin, dass eine Verschlechterung der finanziellen Situation mit einer schlechteren subjektiven Gesundheit einhergeht (Piumatti 2017; Giordano/Lindstrom 2010). Nur eine der Untersuchungen analysiert auch den möglichen Effekt einer besseren finanziellen Lage, wobei kein signifikanter Zusammenhang mit der subjektiven Gesundheit gefunden wird (Giordano/Lindstrom 2010). Die im vorangegangenen Abschnitt durchgeführten Analysen erweitern somit den bisherigen spärlichen Forschungsstand um neue Erkenntnisse: Zum Teil geht eine Verbesserung der finanziellen Lage mit einer Verbesserung der subjektiv empfundenen Gesundheit einher.

Einen ganz neuen Aspekt liefert zudem die Betrachtung der Einschränkungen in den ADL. Dieser Indikator der physischen Gesundheit wurde bislang noch nicht auf diese Weise untersucht. Da die Mobilität und Selbstständigkeit für die Lebenszufriedenheit von älteren Menschen eine wichtige Rolle spielt (vgl. Andersen et al. 2004; Netuveli et al. 2006), ist es eine wichtige Erkenntnis, dass Veränderungen der finanziellen Lage mit der Entwicklung von körperlichen Einschränkungen in Zusammenhang stehen. Wie bereits in Kapitel 5.5 diskutiert, kann allerdings nicht ausgeschlossen werden, dass hier eine umgekehrte Kausalität vorliegt. Es sind Effekte in beide Richtungen möglich. Stellt eine finanzielle Krise eine Stresssituation dar, kann dies ein Auslöser von 
Herzinfarkt oder Schlaganfall sein (Steptoe/Kivimäki 2013), was als Folge körperliche Beeinträchtigungen nach sich ziehen kann. Andererseits können Einschränkungen in ADL mit Kosten verbunden sein (z.B. Hilfsmittel, Pflege), die sich auf die finanzielle Lage auswirken. Wie stark die entgegengesetzten Effekte sind, kann hier nicht geklärt werden. Daher sollten die Analysen der Einschränkungen in ADL mit Vorsicht interpretiert werden. Unabhängig davon ist es eine wichtige Feststellung, dass durchaus auch im höheren Alter Verbesserungen bei körperlichen Einschränkungen zu beobachten sind (vgl. auch Hardy/Gill 2004).

Ein Vergleich der Effektstärken von Verbesserung und Verschlechterung der finanziellen Situation wurde bislang nur in Studien zu Depressionen durchgeführt. In der Untersuchung von Lorant et al. (2007) wurde für einen der untersuchten Armutsindikatoren (Probleme mit dem Einkommen auszukommen) festgestellt, dass die Effektstärke bei Verschlechterung größer ist als bei einer Verbesserung der finanziellen Lage. Mckenzie et al. (2014) haben hingegen keine signifikanten Unterschiede in den Effektstärken von Verbesserung und Verschlechterung festgestellt. In den hier durchgeführten Berechnungen konnten vereinzelte signifikante Unterschiede zwischen dem (Betrag der) Koeffizienten von Verbesserung der finanziellen Lage und dem entgegengesetzten Koeffizienten der Verschlechterung beobachtet werden: Zweimal zeigt sich ein stärkerer Effekt bei Verbesserung (Depression und subjektive Gesundheit) und einmal ein stärkerer Effekt bei Verschlechterung (subjektive Gesundheit). Insgesamt deuten die Ergebnisse jedoch darauf hin, dass die (Beträge der) Effekte bei Verbesserung und Verschlechterung etwa gleich stark sind. Die von der Theorie der Verlustaversion abgeleitete Vermutung, dass Verlust, bzw. eine Verschlechterung der finanziellen Lage, stärkere negative Auswirkungen hat als eine entsprechende Verbesserung zu positiven Auswirkungen führt, kann somit nicht bestätigt werden. Die FE-Modelle können also so interpretiert werden, dass Effekte in beide Richtungen vorliegen. Zudem sind die Effektstärken in den FD-Modellen denen der FE-Modelle sehr ähnlich. Änderungen der finanziellen Situation beeinflussen die Wahrscheinlichkeit von schlechter Gesundheit um ca. 3 bis 6 Prozentpunkte. Die FD-Analysen führen somit zu der Schlussfolgerung, dass die Effekte von Veränderungen in der finanziellen Situation auf die Gesundheit mit den vorliegenden Daten anhand von Berechnungen mit FE-Modellen angemessen beschrieben werden.

Die genauere Betrachtung des Einflusses von Verschuldung bestätigt die Ergebnisse der Analysen zur finanziellen Situation nur teilweise. Nur auf Depressionen wirken sich neue Schulden negativ aus, und eine vollständige Tilgung von Schulden ist mit einem geringeren Depressionsrisiko verknüpft. Dies entspricht den bisherigen Ergebnissen der Studien zum Zusammenhang von Schulden und Depressionen (Richardson/Elliott/Roberts 2013; Hiilamo/Grundy 2018). Für Einschränkungen in ADL und subjektive Gesundheit 
kann hingegen kein signifikanter Zusammenhang mit einer neuen Verschuldung aufgezeigt werden. Die Koeffizienten bei Tilgung der Schulden sind zwar signifikant unterschiedlich im Vergleich zur Referenzkategorie, allerdings nicht verschieden von den Koeffizienten für Schulden zu beiden Zeitpunkten. Dass nur signifikante Effekte für Depression, aber nicht für die Maße physischer Gesundheit zu beobachten sind, könnte durch die angesprochene Latenzzeit begründet sein.

In den Analysen taucht ein unplausibles Ergebnis auf, das bereits in der deskriptiven Analyse in Kapitel 5.1.6 beobachtet wurde. Den Berechnungen zufolge ist es für die (Entwicklung der) Gesundheit besser Schulden zu haben als keine Schulden zu haben. Es gibt theoretische Erklärungen, warum Schulden mit besserem Wohlbefinden verknüpft sind. Demnach können durch relativ geringe und kurzfristige Schulden Schwankungen im Einkommen und der wirtschaftlichen Gesamtlage ausgeglichen werden, wodurch der Konsum auch in Krisen konstant gehalten werden kann (Clayton/Liñares-Zegarra/Wilson 2015). Schulden können zudem langfristige Investitionen ermöglichen und sich dadurch positiv auf das Wohlbefinden auswirken (Berger/Collins/Cuesta 2016). 


\section{Analysen: Einkommens- und Vermögensungleichheit}

Dass Veränderungen in der finanziellen Situation des Haushaltes mit Veränderungen der mentalen und physischen Gesundheit einhergehen, wurde in den vorherigen Kapiteln dargestellt. In diesem Kapitel werden weitere ökonomische Aspekte in die Analysen integriert: die ökonomischen Rahmenbedingungen der Gesellschaft, in der die Befragten leben. Im Zentrum steht hierbei die ungleiche Verteilung des Einkommens und des Vermögens. Dabei werden auch Indikatoren kontrolliert und getestet, die die psychosoziale und neomaterielle Theorie repräsentieren und den Zusammenhang von Ungleichheit und Gesundheit erklären könnten (siehe Kapitel 2.2.8). Zudem wird untersucht, ob die Folgen für die Gesundheit, die mit einer Veränderung in der individuellen finanziellen Situation einhergehen, auch von dem Ungleichheitsniveau der Gesellschaft beeinflusst werden. Die hier durchgeführte Analyse ist dabei eine der ersten, die die Ungleichheit im Vermögen in diesem Zusammenhang berücksichtigt.

Zunächst folgen in Kapitel 7.1 die deskriptive Beschreibung der Indikatoren sowie deren bivariate Korrelationen über die untersuchten Länder hinweg. In Kapitel 7.2 wird in multivariaten Analysen untersucht wie das Niveau und die Veränderung der Ungleichheit die individuelle Gesundheit beeinflusst. Hierbei werden auch mögliche Mechanismen untersucht, die den gängigen Theorien zufolge für die Verknüpfung von Gesundheit und Ungleichheit verantwortlich sind. Zudem wird der Frage nachgegangen, ob die gesundheitlichen Folgen, die aus einer Veränderung der individuellen finanziellen Situation resultieren, in allen Gesellschaften gleich sind oder nach dem Ausmaß der vorherrschenden Ungleichheit variieren. Im Anschluss an die Analysen folgt die Diskussion der Ergebnisse in Kapitel 7.3. Eine Einordnung der Ergebnisse ist hier von besonderer Bedeutung, da einerseits zu einigen der Fragestellungen bislang kein oder ein nur sehr kleiner Forschungsstand existiert und andererseits eine Methode Verwendung findet, die in diesem Forschungsfeld bisher noch nicht angewandt wurde.

\subsection{Deskriptive Beschreibungen}

Die in dieser Arbeit analysierten Länder unterscheiden sich in vielerlei Hinsicht voneinander. Dies wird nicht zuletzt anhand der ökonomischen Indikatoren deutlich, die in diesem Kapitel im Mittelpunkt stehen. Tabelle 7.1 zeigt diese zum Zeitpunkt der aktuellsten SHARE Daten, der sechsten Welle aus 
dem Jahr 2015. ${ }^{18}$ Die Ungleichheit im Einkommen, gemessen anhand des GiniKoeffizienten $^{19}$, reicht von 24,5 in Slowenien bis zu 36,0 in Israel. In der Gruppe der Länder mit der höchsten Ungleichverteilung des Einkommens befinden sich mit Ausnahme Estlands nur die südlich gelegenen Länder - Italien, Portugal, Spanien, Griechenland und Israel. Die Ungleichheit im Vermögen ist in allen Ländern deutlich größer. Auch hier hat Slowenien mit 58,5 den niedrigsten Wert. Die größte Vermögensungleichheit herrscht mit einem Gini-Koeffizienten von 89,3 in Dänemark. Die Werte auf der Skala des sozialen Vertrauens variieren zwischen 12,3 in Polen und 20,2 in Dänemark. Eine beachtliche Spannweite kann in dem Anteil der privaten Gesundheitsausgaben an den gesamten Gesundheitsausgaben in den Ländern beobachtet werden. In Frankreich muss die Bevölkerung mit Abstand am wenigsten zur Gesundheitsversorgung beisteuern, nur 6,8 Prozent wurden hier im Jahr 2015 aus eigener Tasche bezahlt. Die Bürger in Portugal und der Schweiz müssen sich in großem Maße, mit ca. 28 Prozent, an den Gesundheitskosten beteiligen. Mit Abstand am höchsten ist die Selbstbeteiligung an den Gesundheitskosten mit über einem Drittel in Griechenland.

Bei allen Indikatoren sind die Veränderungen im Zeitverlauf relativ gering im Vergleich zu den Niveauunterschieden, die zwischen den Ländern vorzufinden sind (vgl. Onlineanhang 35). Für die Entwicklung der Ungleichheit im Einkommen kann kein gemeinsamer Trend der untersuchten Länder festgestellt werden. Relativ starke Anstiege des Gini-Koeffizienten sind im jeweiligen Untersuchungszeitraum in Schweden $(3,7)$, Spanien $(3,6)$ und Dänemark (3,5, jeweils von 2004 bis 2015) sowie in Estland (3,5 von 2010 bis 2015), zu beobachten. Die stärksten Rückgänge zeigen sich in Polen (-2,7 von 2006 bis 2015) und den Niederlanden (-2,5 von 2004 bis 2013). Auch der Index des sozialen Vertrauens (mögliche Werte von 0 bis 30) ist insgesamt in den einzelnen Ländern recht stabil. Die stärkste Schwankung liegt in Slowenien vor, zwischen dem geringsten $(12,85)$ und dem höchsten Wert $(14,65)$ liegen 1,8 Skalenpunkte. Der stärkste Anstieg des sozialen Vertrauens im Untersuchungszeitraum zeigt sich in Deutschland, von 15,37 im Jahr 2004/2005 auf 16,49 im Jahr 2014/2015. Der deutlichste Rückgang ist in Estland zu beobachten, von 17,05 im Jahr 2010/2011 auf 16,44 im Jahr 2014/2015. Ebenfalls relativ konstant im Zeitverlauf ist das Verhältnis der privaten und staatlichen Gesundheitsausgaben. Die stärkste Veränderung im Untersuchungszeitraum ist der Rückgang des Anteils der privaten Gesundheitsausgaben um 5,9 Prozentpunkte in Israel (2005-2015). Auch in Polen (-3,9 Prozentpunkte von 2006 bis 2015) und der Schweiz (-3,5 Prozentpunkte, 2004-2015) hat sich das Verhält-

18 Eine Ausnahme hiervon sind die Werte der Vermögensungleichheit, die nur zu einem früheren Zeitpunkt vorliegen (siehe Kapitel 4.3.2).

19 Für eine bessere Lesbarkeit der Ergebnisse wurden die Werte des Gini-Koeffizienten, die von 0 bis 1 variieren können, mit 100 multipliziert. 
nis hin zu geringeren privaten Gesundheitsausgaben entwickelt. In den Niederlanden (2004 bis 2013) und Tschechien (2006 bis 2015) hat sich der Anteil der Kosten, die die Bevölkerung selbst für die Gesundheitsversorgung aufbringen muss, um 3,8 bzw. 3,2 Prozentpunkte erhöht. Die Werte aller Indikatoren von allen Ländern zu allen Beobachtungszeitpunkten sind im Onlineanhang $35 \mathrm{zu}$ finden.

Tabelle 7.1: Makroindikatoren

\begin{tabular}{|c|c|c|c|c|c|c|}
\hline & $\begin{array}{l}\text { Einkom- } \\
\text { mensun- } \\
\text { gleichheit }\end{array}$ & $\begin{array}{l}\text { Vermö- } \\
\text { gensun- } \\
\text { gleichheit }\end{array}$ & $\begin{array}{l}\text { Soziales } \\
\text { Ver- } \\
\text { trauen }\end{array}$ & $\begin{array}{l}\text { Anteil priva- } \\
\text { ter Gesund- } \\
\text { heitsausga- } \\
\text { ben }\end{array}$ & BIP & $\begin{array}{l}\text { BIP } \\
\text { logarithmiert }\end{array}$ \\
\hline & $\begin{array}{l}\text { Gini x } 100 \\
(0-100)\end{array}$ & $\begin{array}{l}\text { Gini x } 100 \\
(0-100)\end{array}$ & $\begin{array}{l}\text { Trust } \\
\text { Scale } \\
(0-30)\end{array}$ & Prozent & $\begin{array}{l}\text { zu konstanten } \\
\text { Preisen, kauf- } \\
\text { kraftbereinigt }\end{array}$ & $\begin{array}{l}\text { zu konstanten } \\
\text { Preisen, kauf- } \\
\text { kraft-bereinigt }\end{array}$ \\
\hline Österreich & 27,2 & 78,5 & 15,8 & 17,9 & $43.052,6$ & 10,7 \\
\hline Deutschland & 30,1 & 78,9 & 16,5 & 12,5 & $42.671,0$ & 10,7 \\
\hline Schweden & 26,7 & 83,2 & 19,1 & 15,2 & $44.323,3$ & 10,7 \\
\hline Spanien & 34,6 & 68,0 & 14,5 & 24,2 & $31.796,1$ & 10,4 \\
\hline Italien & 32,4 & 68,7 & & 22,8 & $33.203,9$ & 10,4 \\
\hline Frankreich & 29,2 & 72,0 & 15,2 & 6,8 & $36.823,0$ & 10,5 \\
\hline Dänemark & 27,4 & 89,3 & 20,2 & 13,7 & $44.524,2$ & 10,7 \\
\hline Griechenland & 34,2 & 67,0 & & 35,5 & $23.729,9$ & 10,1 \\
\hline Schweiz & 29,6 & 72,1 & 17,7 & 28,4 & $54.500,7$ & 10,9 \\
\hline Belgien & 26,2 & 64,1 & 15,5 & 17,6 & $40.829,3$ & 10,6 \\
\hline Israel & 36,0 & 77,2 & 15,6 & 24,4 & $31.228,3$ & 10,4 \\
\hline Tschechien & 25,0 & 76,0 & 14,4 & 14,8 & $29.932,0$ & 10,3 \\
\hline Polen & 30,6 & 73,0 & 12,3 & 23,3 & $24.186,1$ & 10,1 \\
\hline Luxemburg & 28,5 & 75,4 & & 10,6 & $88.003,4$ & 11,4 \\
\hline Portugal & 34,0 & 71,3 & 12,8 & 27,6 & $26.701,9$ & 10,2 \\
\hline Slowenien & 24,5 & 58,5 & 13,9 & 12,5 & $28.144,4$ & 10,3 \\
\hline Estland & 34,8 & 65,6 & 16,4 & 22,8 & $26.237,8$ & 10,2 \\
\hline
\end{tabular}

Quelle: Eurostat (2016), OECD (2018a; 2018b; 2016), Credit Suisse Research Institute (2016), ESS (2014). Werte für 2015, außer Vermögensungleichheit (verschiedene Jahre, siehe Kapitel 4.3.2). Onlineanhang 35 bietet eine Übersicht über alle Indikatoren im Zeitverlauf.

Betrachtet man die Ungleichheiten im Einkommen und im Vermögen in Relation zueinander, so ergibt sich das in Abbildung 7.1 dargestellte Bild. Die eingezeichnete Regressionsgerade deutet nicht auf einen starken Zusammenhang zwischen beiden Ungleichheitsdimensionen hin. Die Regression ist jedoch 
stark von den beiden Ausreisern Belgien und Slowenien beeinflusst. Beide Länder weisen eine geringe Ungleichheit sowohl im Vermögen, als auch im Einkommen auf. Die übrigen Länder zeigen durchaus eine Korrelation: Mit größerer Ungleichheit im Einkommen in einem Land geht tendenziell eine geringere Ungleichheit im Vermögen einher.

Abbildung 7.1: Korrelation von Einkommensungleichheit (2015) und Vermögensungleichheit

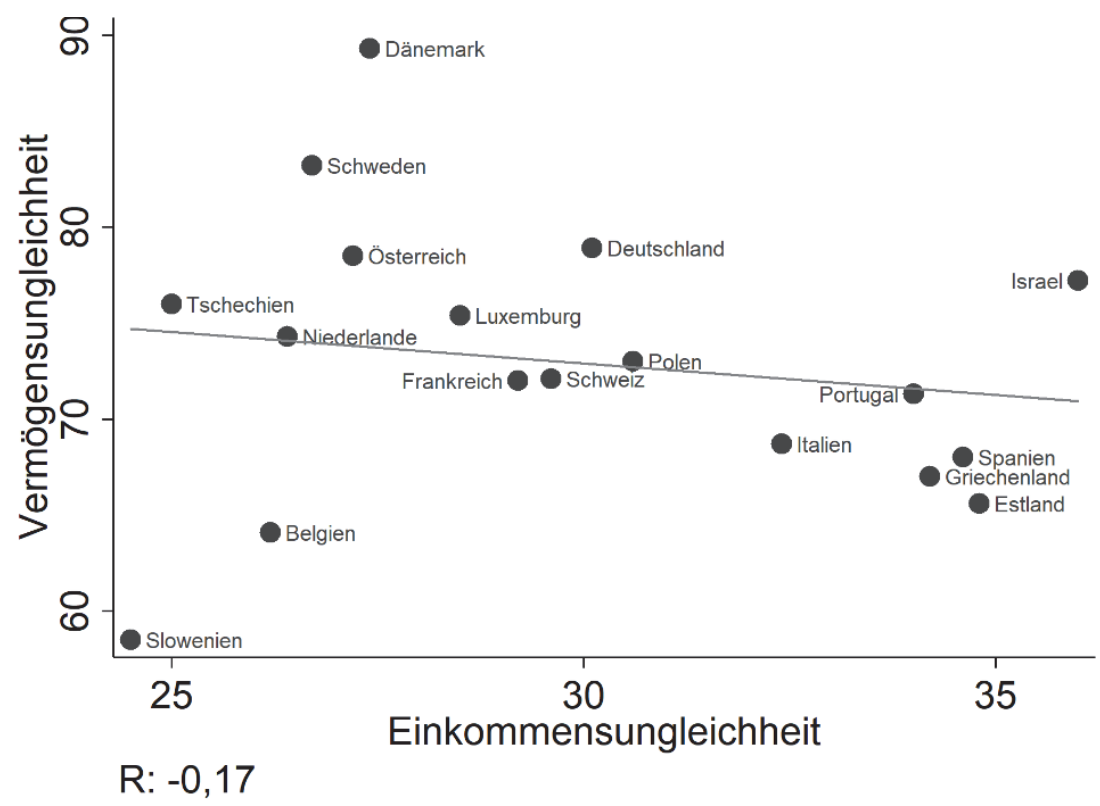

Quelle: Eurostat (2016), OECD (2016), Credit Suisse Research Institute (2016). Siehe Onlineanhang 35. Eigene Darstellung.

Anmerkung: Obwohl keine Interviewdaten aus den Niederlanden von der SHARE Welle 6 vorliegen, ist die Niederlande in dieser Grafik mit den Informationen zum Zeitpunkt der SHARE Welle 6 (2015) enthalten.

Abbildung 7.2 bietet eine Übersicht über die bivariaten Korrelationen aller Indikatoren auf Länderebene. Verwendet werden auch hierfür die Daten zum Zeitpunkt der letzten SHARE Befragung (Welle 6) aus dem Jahr 2015. Jeder Punkt im Koordinatensystem stellt ein Land dar, eine lineare Regressionsgerade ist eingezeichnet und unter der Grafik ist die Korrelation (R) angegeben. Die erste Zeile zeigt die Korrelationen mit der Einkommensungleichheit. Eine größere Einkommensungleichheit geht mit geringerem sozialem Vertrauen, einem höheren Anteil privater Gesundheitsausgaben sowie mit geringerem BIP 
pro Person (logarithmiert) einher. Für die Vermögensungleichheit - die zweite Zeile in der Abbildung - sind die Zusammenhänge mit den drei Maßen jeweils entgegengesetzt. In der dritten Zeile sind die Korrelationen der restlichen Kombinationen der Indikatoren dargestellt. Besonders stark ist die Korrelation von logarithmiertem BIP pro Person und sozialem Vertrauen: Je größer die Wirtschaftskraft eines Landes ist, umso größer ist das Vertrauen in die Mitmenschen. Der Anteil der privaten Gesundheitsausgaben an den gesamten Gesundheitsausgaben eines Landes ist tendenziell geringer, je höher das BIP pro Person ist.

Abbildung 7.2: Korrelation der Makroindikatoren
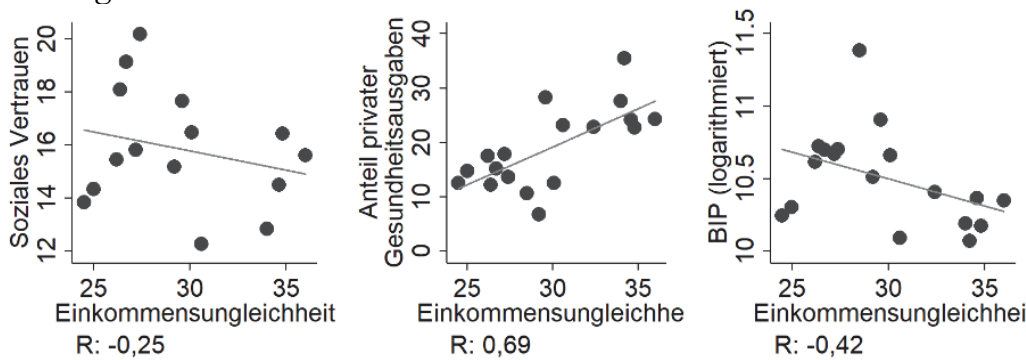

Einkommensungleichhe

R: 0,69

Einkommensungleichheit

R: $-0,42$
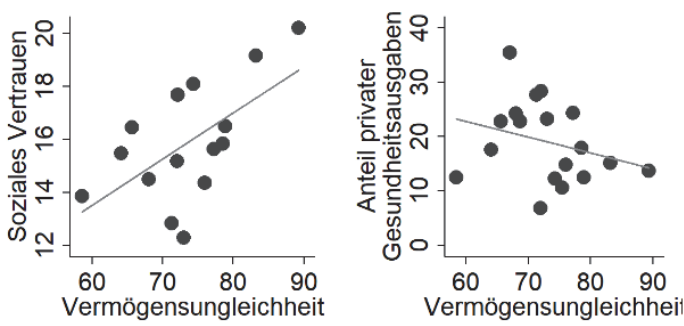

R: 0,6

R: $-0,28$
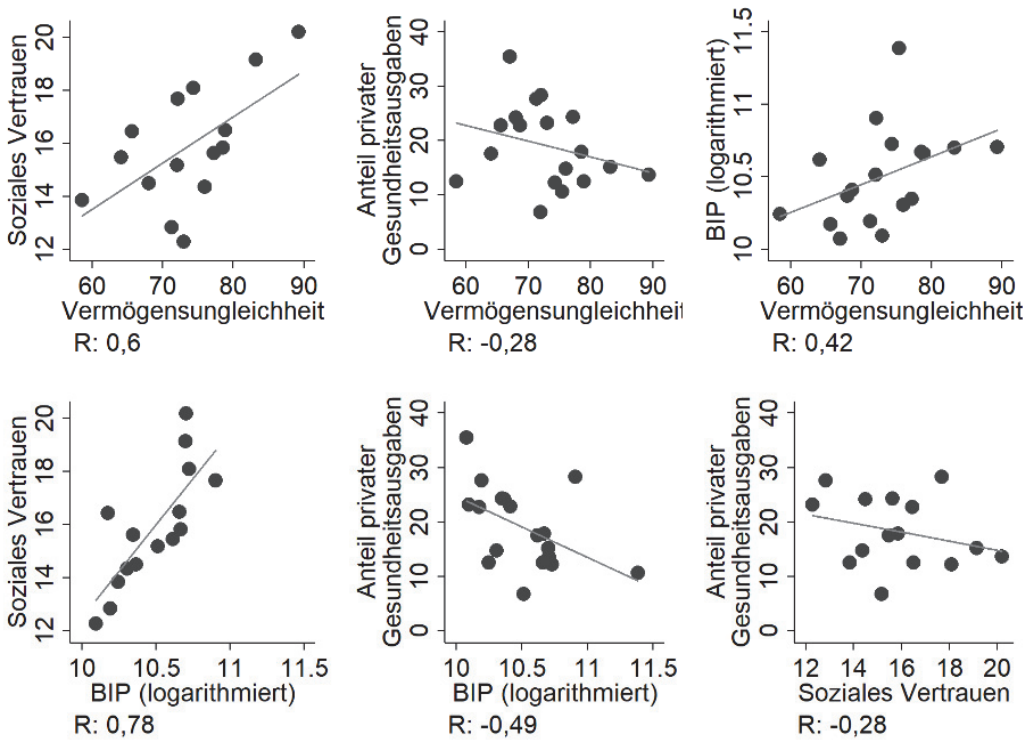

Quelle: Eurostat (2016), OECD (2018a; 2018b), ESS (2014), Credit Suisse Research Institute (2016). Eigene Darstellung. Alle Werte für das Jahr 2015, außer Vermögensungleichheit (siehe Kapitel 4.3.2). Für die genauen Werte siehe Onlineanhang 35. 

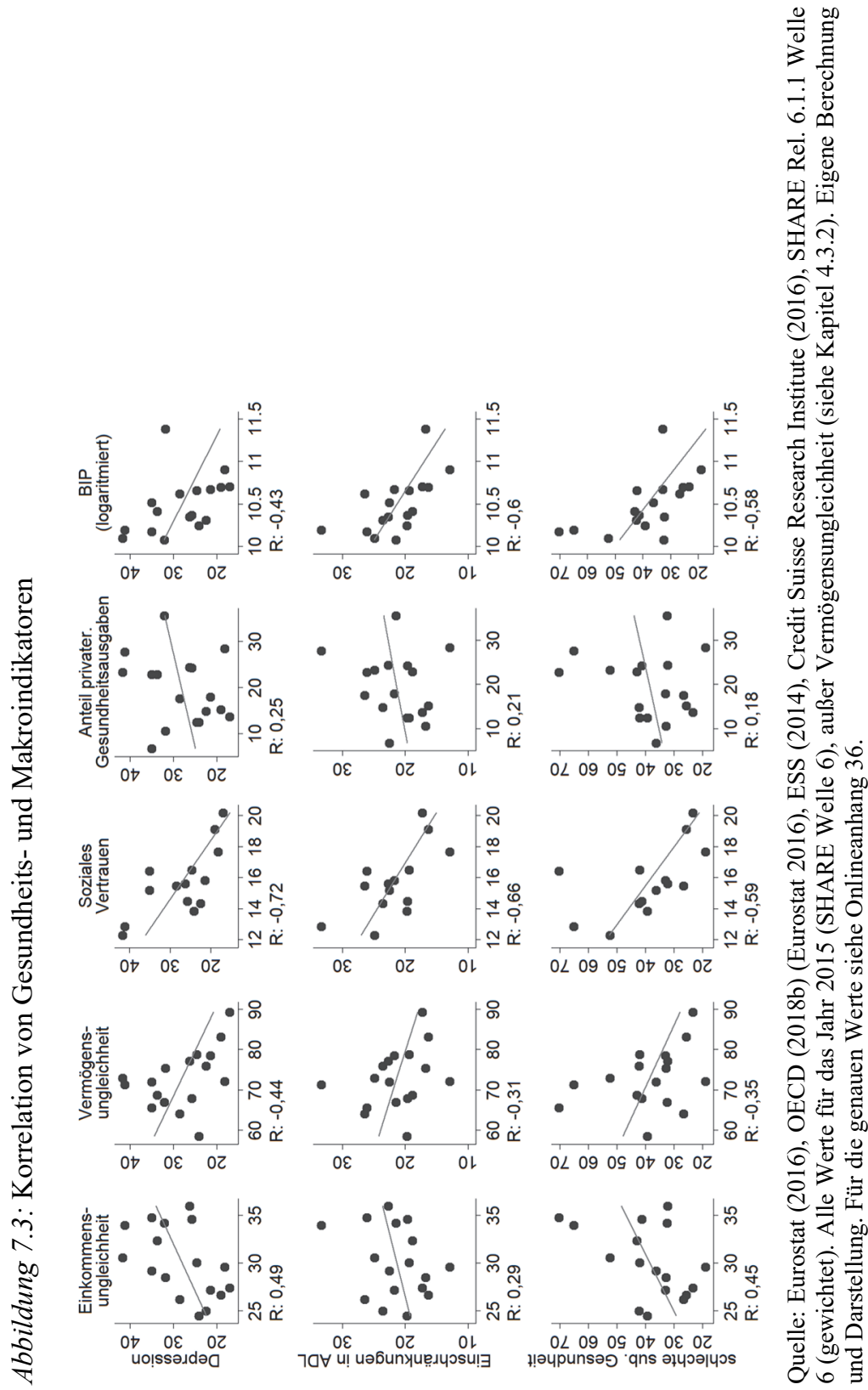
Für die Fragestellung dieses Kapitels - v.a. für die Überprüfung der neomateriellen und psychosozialen Theorie - sind die beschriebenen Korrelationen der Makroindikatoren untereinander von Bedeutung. Der Kern der Untersuchung ist jedoch ihre Korrelation mit der Gesundheit der Bevölkerung. Diese ist anhand von gewichteten Informationen aus SHARE Welle 6 in Abbildung 7.3 dargestellt. Da Einkommens- und Vermögensungleichheit negativ korreliert sind, sind auch hier die Korrelationen mit den Gesundheitsindikatoren gegenläufig. Während größere Ungleichheit im Einkommen mit schlechterer Gesundheit der Bevölkerung einhergeht, ist größere Vermögensungleichheit mit besserer Gesundheit verknüpft.

Wie in der psychosozialen Hypothese beschrieben, besteht ein deutlicher Zusammenhang von sozialem Vertrauen und Gesundheit der Bevölkerung. Für alle untersuchten Gesundheitsindikatoren gilt: Je größer das Vertrauen in die Mitmenschen in einem Land ist, umso geringer ist der Anteil der Bevölkerung ab 50 Jahren mit schlechter Gesundheit. Der in der neomateriellen Hypothese beschriebene Zusammenhang - schlechtere Gesundheit der Bevölkerung bei weniger staatlichen Investitionen - ist mit den hier verwendeten Indikatoren hingegen nicht stark ausgeprägt. Aus Abbildung 7.3 geht zudem hervor, dass in der Altersgruppe ab 50 Jahren der Anteil der Personen mit schlechter Gesundheit tendenziell geringer ist, je größer die Wirtschaftskraft eines Landes ist.

Die Überlegung, dass die Gesundheit der Bevölkerung nicht von dem aktuellen Grad der Ungleichheit in einer Gesellschaft abhängt, sondern vielmehr Ungleichheiten aus früheren Lebensphasen ihre Spuren hinterlassen haben, wird hier anhand bivariater Korrelationen auf Länderebene untersucht. Abbildung 7.4 zeigt die Korrelation der drei Gesundheitsindikatoren aus SHARE Welle 6 mit den Gini-Koeffizienten der Einkommensungleichheit zu verschiedenen Zeitpunkten, nämlich im selben Jahr sowie 5, 10 und 15 Jahre zuvor. Je weiter die Messung der Ungleichheit zurückgeht, umso weniger Länder stehen für die Analyse zur Verfügung. Erst seit 2005 wird von allen EU-Ländern die Einkommensungleichheit jährlich an die EU berichtet (EU 2003). Die Grafiken zeigen, dass die Korrelationen bei einer verzögerten Messung der GiniKoeffizienten um 10 und 15 Jahre deutlich stärker ausfallen, als unter Verwen dung aktuellerer Werte. Während bei den Einschränkungen in den ADL mit der Korrelation auch die Steigung der Regressionsgeraden zunimmt, ist dies bei den beiden anderen Gesundheitsmaßen nicht der Fall. Bei Depression bleibt die Steigung der Regressionsgerade über alle Grafiken hinweg ungefähr gleich. Bei subjektiver Gesundheit ist hingegen kein eindeutiges Muster zu erkennen. 
Abbildung 7.4: Korrelation von Gesundheit und Einkommensungleichheit bei verzögerter Messung der Einkommensungleichheit
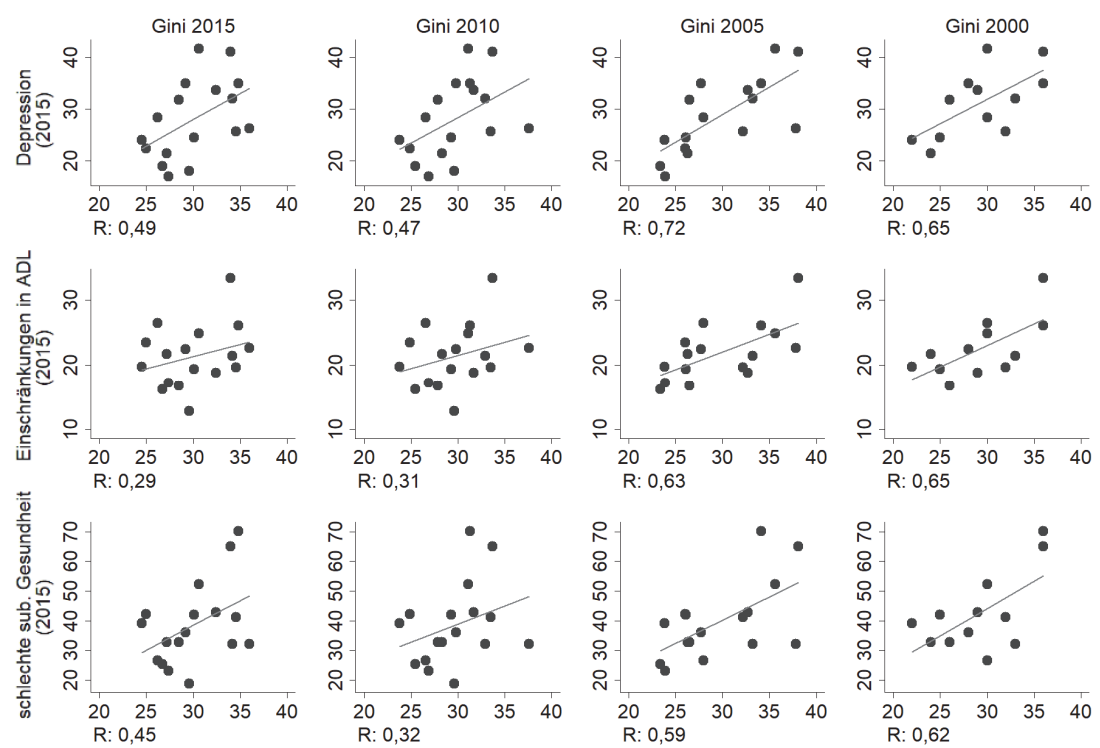

Quelle: SHARE Rel. 6.1.1, Welle 6, eigene Berechnungen, gewichtet, Werte siehe Onlineanhang 36. Eurostat (2016), Werte in Onlineanhang 37.

\subsection{Multivariate Analysen}

Um zu testen, wie ökonomische Ungleichheit einer Gesellschaft mit individueller Gesundheit in Verbindung steht, kommen Hybrid-Modelle zur Anwendung. Auf diese Weise können sowohl Within-Schätzer, als auch Between-Schätzer in einem Modell berechnet werden (siehe Kapitel 4.6.5). Hybrid-Modelle erlauben zudem die Aufnahme von zeitkonstanten Variablen und berechnen deren Between-Schätzer. Da für die Vermögensungleichheit keine zeitveränderlichen Daten zur Verfügung stehen, ist es also möglich, Koeffizienten für die Vermögensungleichheit (Between) und die Einkommensungleichheit (Between und Within) im selben Modell zu schätzen. Die WithinSchätzer sind identisch mit den FE-Schätzern und bilden somit den Effekt von Veränderung ab. Die Between-Schätzer können als Niveauunterschiede interpretiert werden (siehe dazu die Diskussion Kapitel 7.3). 
Die Stichprobe, die für diese Analysen verwendet wird, ist kleiner als die Stichprobe der FE-Analysen. Grund dafür sind fehlende Informationen für die Indikatoren auf Länderebene. Es sind keine Daten verfügbar für die Einkommensungleichheit in Deutschland, den Niederlanden und der Schweiz (Welle 1), für den Anteil privater Gesundheitsausgaben in Griechenland (Wellen 1 und 2) sowie für das soziale Vertrauen in Italien (Wellen 1, 2, 4, 6), Österreich (Wellen 4 und 5), Griechenland (Wellen 2 und 6), Israel (Welle 1) und Luxemburg (Wellen 5 und 6). Dies hat zur Folge, dass Griechenland und Luxemburg nicht in die Berechnungen in diesem Kapitel einbezogen werden können. Von den Daten aus Italien bleibt nur eine Welle übrig, so dass die italienischen Daten in die Between- aber nicht in die Within-Schätzer einfließen. Abgesehen von der Zusammensetzung nach Ländern, weicht die Stichprobe kaum von der ursprünglichen Stichprobe ab (siehe Onlineanhang 38).

In Tabelle 7.2 sind die Ergebnisse des Hybrid-Modells für alle Gesundheitsindikatoren dargestellt. Daraus wird ersichtlich, dass mit einem höheren Niveau an Vermögensungleichheit eine geringere Wahrscheinlichkeit von Depression verbunden ist. Der entgegengesetzte Zusammenhang lässt sich bei der Einkommensungleichheit beobachten. Sowohl ein höheres Niveau an Ungleichheit, als auch eine Erhöhung der Ungleichheit im Zeitverlauf, gehen mit einer höheren Depressionswahrscheinlichkeit einher. Zudem zeigt sich, dass bei einem höheren Niveau und mit einem Anstieg des Anteils der privaten Gesundheitsausgaben die Depressionswahrscheinlichkeit geringer ist. Dies widerspricht dem (schwachen) entgegengesetzten Zusammenhang, der in der bivariaten Betrachtung der Indikatoren auf Länderebene zu sehen ist (vgl. Abbildung 7.3). Dass mit einem höheren Niveau an sozialem Vertrauen die Wahrscheinlichkeit an Depression zu leiden geringer wird, entspricht hingegen den vorangegangenen Analysen. Veränderungen im sozialen Vertrauen scheinen jedoch nicht mit Depressionen in Zusammenhang zu stehen.

Die Einschränkungen in Aktivitäten des täglichen Lebens stehen nicht in Zusammenhang mit der Vermögensungleichheit. Bei der Einkommensungleichheit ist nur das Niveau, aber nicht ihre Veränderung, mit den Einschränkungen in ADL verknüpft. Das Vorzeichen ist jedoch anders als erwartet: Je größer die Ungleichheit, desto geringer ist die Wahrscheinlichkeit an mindestens einer Einschränkung zu leiden. Weder der Anteil der privaten Gesundheitsausgaben, noch das soziale Vertrauen, ist mit den individuellen körperlichen Einschränkungen verknüpft.

In der Analyse der subjektiven Gesundheit zeigt sich ebenfalls kein Zusammenhang mit der Vermögensungleichheit. Im Gegensatz dazu ist eine größere Einkommensungleichheit mit einer höheren Wahrscheinlichkeit von schlechter subjektiver Gesundheit verbunden, das gilt sowohl für das Niveau als auch für Veränderungen. Die Richtung des Zusammenhangs von sozialem Vertrauen und subjektiver Gesundheit widerspricht den theoretischen Argumen- 
ten, denn der Berechnung zufolge geht hohes soziales Vertrauen mit schlechterer Gesundheit einher. Für den Anteil von privaten Gesundheitsausgaben ist nur ein negativer Niveaueffekt zu beobachten: Ein hohes Niveau des Anteils der privaten Gesundheitskosten geht mit einer geringeren Wahrscheinlichkeit schlechter subjektiver Gesundheit einher.

\subsubsection{Test der psychosozialen und neomateriellen Theorie}

Die vermuteten Zusammenhänge von Ungleichverteilung im Einkommen und Gesundheit konnten anhand von zwei der drei Indikatoren - Depression und subjektive Gesundheit - bestätigt werden. Die beiden theoretischen Begründungen für diesen Zusammenhang werden dabei mit jeweils einem Indikator berücksichtigt. Das soziale Vertrauen als Mediator ist aus der psychosozialen Theorie abgeleitet. Die neomaterielle Theorie argumentiert, dass die staatlichen Investitionen - hier in Bezug auf die Gesundheitsversorgung anhand des Anteils der privaten Gesundheitsausgaben gemessen - als Ursache für gesundheitliche Unterschiede verantwortlich sind. Um die Relevanz der Theorien zu testen, werden die Berechnungen aus Tabelle 7.2 wiederholt und die Modelle verglichen, die mit und ohne Kontrolle der beiden Indikatoren berechnet werden (siehe Onlineanhang 39 bis 41). Bei den Analysen deutet nichts darauf hin, dass der Zusammenhang von Einkommensungleichheit und Gesundheit über soziales Vertrauen oder die Gesundheitsausgaben vermittelt ist. Anstatt eines Rückgangs der Korrelation zu beobachten - wie es bei einer Mediation der Fall wäre -, intensiviert sich der Zusammenhang sobald die aus den Theorien abgeleiteten Maße kontrolliert werden. So ist für die Depressionswahrscheinlichkeit bei Kontrolle des Anteils der Gesundheitsausgaben eine deutlichere Korrelation zu erkennen. Bei der subjektiven Gesundheit tragen beide Makroindikatoren dazu bei, dass der Zusammenhang stärker wird.

Was die Korrelation zwischen Gesundheit und Vermögensungleichheit betrifft, so liefern die Berechnungen Hinweise darauf, dass das soziale Vertrauen den Zusammenhang von Vermögensungleichheit und Gesundheit vermittelt. Ohne die Berücksichtigung des sozialen Vertrauens besteht ein positiver $\mathrm{Zu}$ sammenhang zwischen Ungleichheit im Vermögen und schlechter subjektiver Gesundheit. Diese Korrelation verschwindet, sobald das soziale Vertrauen in das Modell mitaufgenommen wird. Das soziale Vertrauen ist selbst signifikant mit der subjektiven Gesundheit verknüpft. Die Richtung des Zusammenhangs ist jedoch, wie oben bereits beschrieben, anders als erwartet - mit größerem Vertrauen geht schlechtere Gesundheit einher. Auch für den Zusammenhang von Vermögensungleichheit und Depression spielt das soziale Vertrauen eine Rolle. Hier führt die Aufnahme ins Modell dazu, dass der negative Zusammenhang von Depression und Vermögensungleichheit schwächer wird. Insgesamt ist das Bild sehr uneinheitlich. Die Indikatoren, die aus den Theorien abgeleitet 
sind, korrelieren teilweise mit der Gesundheit, aber nicht systematisch. Zudem tragen sie nur im Fall von Vermögensungleichheit und subjektiver Gesundheit zur Erklärung des Zusammenhangs von Ungleichheit und Gesundheit bei.

Tabelle 7.2: Hybrid-Modelle

\begin{tabular}{|c|c|c|c|c|c|c|}
\hline \multirow[b]{2}{*}{$\begin{array}{l}\text { Gini Vermögens- } \\
\text { ungleichheit }\end{array}$} & \multicolumn{2}{|l|}{ Depression } & \multicolumn{2}{|c|}{ Einschränkung in ADL } & \multicolumn{2}{|c|}{$\begin{array}{l}\text { Schlechte subjektive } \\
\text { Gesundheit }\end{array}$} \\
\hline & $-0,0009^{* * *}$ & $(0,000)$ & 0,0001 & $(0,000)$ & $-0,0004$ & $(0,000)$ \\
\hline $\begin{array}{l}\text { Gini Einkommens- } \\
\text { ungleichheit (between) }\end{array}$ & $0,0062^{* * *}$ & $(0,001)$ & $-0,0031^{* * *}$ & $(0,000)$ & $0,0083^{* * *}$ & $(0,001)$ \\
\hline $\begin{array}{l}\text { Gini Einkommens- } \\
\text { ungleichheit (within) }\end{array}$ & $0,0026^{*}$ & $(0,001)$ & 0,0015 & $(0,001)$ & $0,0060^{\star * *}$ & $(0,001)$ \\
\hline $\begin{array}{l}\text { Soziales Vertrauen } \\
\text { (between) }\end{array}$ & $-0,0091^{* * *}$ & $(0,001)$ & 0,0006 & $(0,001)$ & $0,0192^{* * *}$ & $(0,001)$ \\
\hline $\begin{array}{l}\text { Soziales Vertrauen } \\
\text { (within) }\end{array}$ & 0,0015 & $(0,004)$ & $-0,0003$ & $(0,003)$ & $0,0103^{* *}$ & $(0,004)$ \\
\hline $\begin{array}{l}\text { Anteil privater Ges. } \\
\text { ausg. (between) }\end{array}$ & $-0,0019^{* * *}$ & $(0,000)$ & $-0,0001$ & $(0,000)$ & $-0,0024^{\star * *}$ & $(0,000)$ \\
\hline $\begin{array}{l}\text { Anteil privater Ges. } \\
\text { ausg. (within) }\end{array}$ & $-0,0053^{* * *}$ & $(0,001)$ & 0,0020 & $(0,001)$ & 0,0001 & $(0,001)$ \\
\hline BIP log. (between) & $0,0499^{* * *}$ & $(0,008)$ & $-0,0346^{\star * *}$ & $(0,007)$ & $-0,3770^{\star * *}$ & $(0,009)$ \\
\hline BIP log. (within) & $-0,2207^{\star \star *}$ & $(0,040)$ & $-0,1902^{\star * *}$ & $(0,036)$ & $0,0865^{\star}$ & $(0,038)$ \\
\hline $\begin{array}{l}\text { Große finanzielle } \\
\text { Probleme (between) }\end{array}$ & $0,1678^{* * *}$ & $(0,007)$ & $0,1199^{\star * *}$ & $(0,006)$ & $0,1719^{\star * *}$ & $(0,007)$ \\
\hline $\begin{array}{l}\text { Große finanzielle } \\
\text { Probleme (within) }\end{array}$ & $0,0513^{* * *}$ & $(0,006)$ & $0,0502^{* * *}$ & $(0,006)$ & $0,0338^{* * *}$ & $(0,006)$ \\
\hline $\begin{array}{l}\text { Einige finanzielle } \\
\text { Probleme (between) }\end{array}$ & $0,0681^{* * *}$ & $(0,004)$ & $0,0568^{* * *}$ & $(0,004)$ & $0,1088^{* * *}$ & $(0,005)$ \\
\hline $\begin{array}{l}\text { Einige finanzielle } \\
\text { Probleme (within) }\end{array}$ & $0,0209^{* * *}$ & $(0,004)$ & $0,0141^{* * *}$ & $(0,003)$ & $0,0184^{* * *}$ & $(0,004)$ \\
\hline Beobachtungen & 155.524 & & 155.524 & & 155.524 & \\
\hline Personen & 61.898 & & 61.898 & & 61.898 & \\
\hline $\mathrm{R}^{2}$ within & 0,0292 & & 0,0262 & & 0,0321 & \\
\hline$R^{2}$ between & 0,2570 & & 0,2620 & & 0,3257 & \\
\hline
\end{tabular}

$* \mathrm{p}<0,05 ; * * \mathrm{p}<0,01 ; * * * \mathrm{p}<0,001$

Quelle: SHARE Rel. 6.1.1, Wellen 1, 2, 4, 5, 6. Eigene Berechnungen, Standardfehler in Klammern. Die Modelle enthalten weitere Kontrollvariablen, die vollständigen Tabellen können in Onlineanhang 39, 40 und 41 nachgeschlagen werden.

\subsubsection{Interaktion: Wer leidet unter der Ungleichheit?}

Die Frage nach der Rolle der ökonomischen Ungleichheit für die Gesundheit soll nun mit den Untersuchungen aus den vorherigen Kapiteln kombiniert werden: Unterscheiden sich die gesundheitlichen Folgen von Veränderungen in der individuellen finanziellen Situation je nach Ungleichverteilung ökonomischer Ressourcen? Um diese Frage zu beantworten werden Hybrid-Modelle berechnet, die einen Interaktionsterm der Veränderung der finanziellen Situation (Within) und der durchschnittlichen Einkommensungleichheit (Between) 
enthalten. Die Ergebnisse der Interaktionen sind in den Abbildungen 7.5 bis 7.7 grafisch dargestellt (für die Regressionstabellen siehe Onlineanhang 42). Die X-Achse bildet den Bereich der in der Stichprobe auftretenden Einkommensungleichheit ab (Gini-Koeffizient 23,2 bis 37,6). Die y-Achse gibt die Effektstärken bei einem Übergang von keinen zu einigen und großen finanziellen Problemen bei entsprechenden Gini-Koeffizienten an.

Im Modell für Depression ist der Between-Effekt der Einkommensungleichheit positiv und signifikant. Das bedeutet, dass eine höhere Ungleichverteilung des Einkommens für alle mit einem höheren Depressionsrisiko einhergeht. Abbildung 7.5 stellt die signifikanten Interaktionseffekte dar: Je größer die Ungleichheit im Einkommen ist, desto stärker ist der schädliche Effekt der Verschlechterung der finanziellen Situation. Die Spannweite der möglichen Effektstärken ist dabei relativ groß. Während eine Veränderung der finanziellen Situation bei geringer Ungleichheit keinen Einfluss auf die Depression hat, ist im ungünstigsten Fall - dem Wechsel hin zu großen finanziellen Schwierigkeiten bei sehr hoher Einkommensungleichheit - eine Steigerung des Depressionsrisikos um über 10 Prozentpunkte möglich.

Abbildung 7.5: Hybrid-Modell: Effekte von Veränderungen der finanziellen Situation nach Einkommensungleichheit: Depression

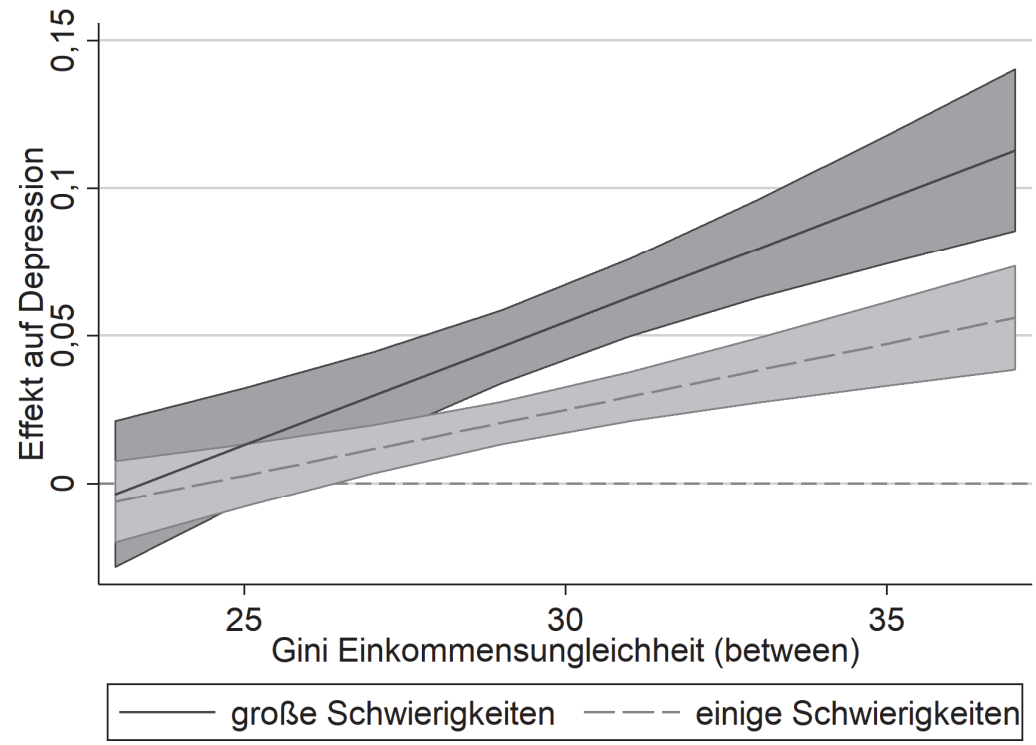

Quelle: SHARE Rel. 6.1.1, Wellen 1, 2, 4, 5, 6. Eigene Berechnungen. Darstellung der Interaktionseffekte und 95\%-Konfidenzintervalle. Die komplette Regressionstabelle befindet sich in Onlineanhang 42. 
Die Analyse mit Einschränkungen in den ADL als abhängiger Variable kommt zu einem etwas anderen Ergebnis. Auch hier zeigt sich, dass der gesundheitsschädliche Effekt umso stärker ist, je größer die finanziellen Probleme werden. Allerdings ist nur der Interaktionseffekt der großen finanziellen Schwierigkeiten signifikant: Je größer die Ungleichheit im Einkommen, desto stärker ist der schädliche Effekt der Verschlechterung hin zu großen finanziellen Schwierigkeiten. Der mit steigender Ungleichheit einhergehende Anstieg des Effektes von einigen finanziellen Schwierigkeiten erweist sich hingegen nicht als signifikant. Dies ist in Abbildung 7.6 grafisch dargestellt. Zu beachten ist jedoch, dass der Haupteffekt der Einkommensungleichheit (Between) negativ und signifikant ist. Das bedeutet, dass insgesamt die Wahrscheinlichkeit an mindestens einer Einschränkung zu leiden mit höherer Ungleichheit im Einkommen bei einem Wechsel zu keinen oder einigen Problemen geringer wird. Der positive Interaktionseffekt bei großen Problemen führt demnach zu einer leicht steigenden Wahrscheinlichkeit einer körperlichen Einschränkung (siehe die Vorhersagewerte in Onlineanhang 43). Es bleibt jedoch festzuhalten: Eine höhere Ungleichheit im Einkommen ist nur schlecht für diejenigen, die große finanzielle Probleme bekommen.

Abbildung 7.6: Hybrid-Modell: Effekte von Veränderungen der finanziellen Situation nach Einkommensungleichheit: Einschränkungen in ADL

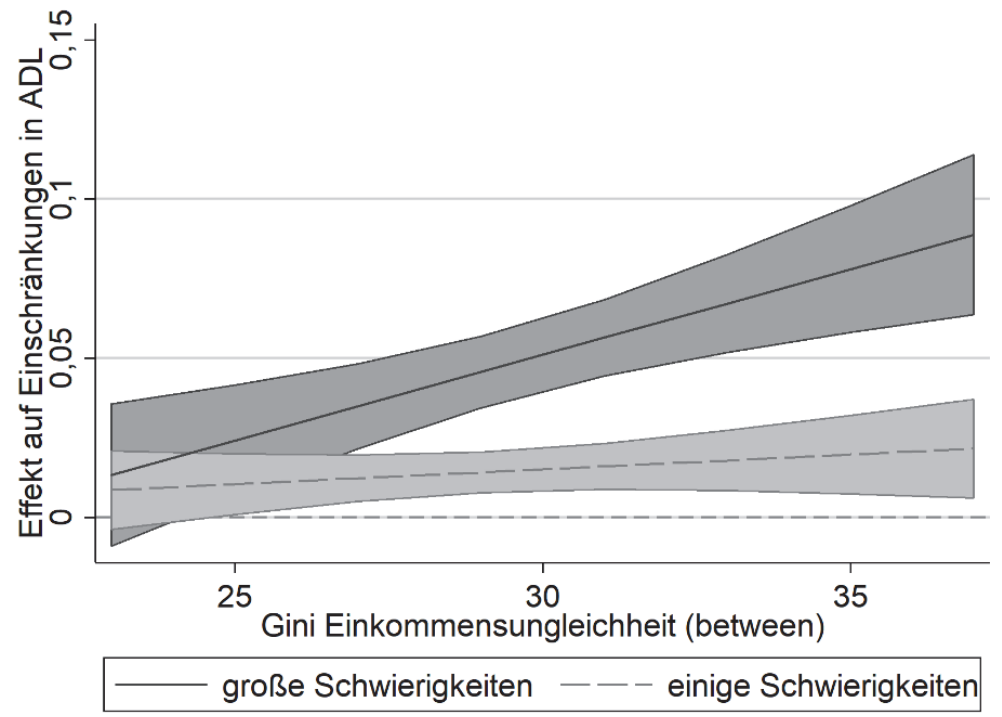

Quelle: SHARE Rel. 6.1.1, Wellen 1, 2, 4, 5, 6. Eigene Berechnungen. Darstellung der Interaktionseffekte und 95\%-Konfidenzintervalle. Die komplette Regressionstabelle befindet sich in Onlineanhang 42. 
In der Analyse zu subjektiver Gesundheit ist, wie auch bei Depression, der Haupteffekt des Niveaus der Einkommensungleichheit positiv. Mit größerer Ungleichheit geht also eine höhere Wahrscheinlichkeit schlechter subjektiver Gesundheit einher. Dieser Anstieg ist bei einigen finanziellen Problemen stärker als bei großen und keinen Problemen. Bei geringer Ungleichheit hat ein Wechsel zu einigen Problemen einen vergleichbaren Effekt auf die subjektive Gesundheit wie ein Wechsel zu keinen Problemen. Mit steigender Ungleichverteilung des Einkommens gleicht sich der Effekt von einigen Problemen dem Effekt von großen Problemen an (siehe auch die Vorhersagewerte in Onlineanhang 43). Für die subjektive Gesundheit lässt sich also feststellen, dass eine Ungleichverteilung im Einkommen für alle schlecht ist- unabhängig von der individuellen finanziellen Situation. Dabei gilt jedoch, dass geringere finanzielle Schwierigkeiten umso stärker ins Gewicht fallen, je größer die Ungleichheit ist.

Abbildung 7.7: Hybrid-Modell: Effekte von Veränderungen der finanziellen Situation nach Einkommensungleichheit: Schlechte subjektive Gesundheit

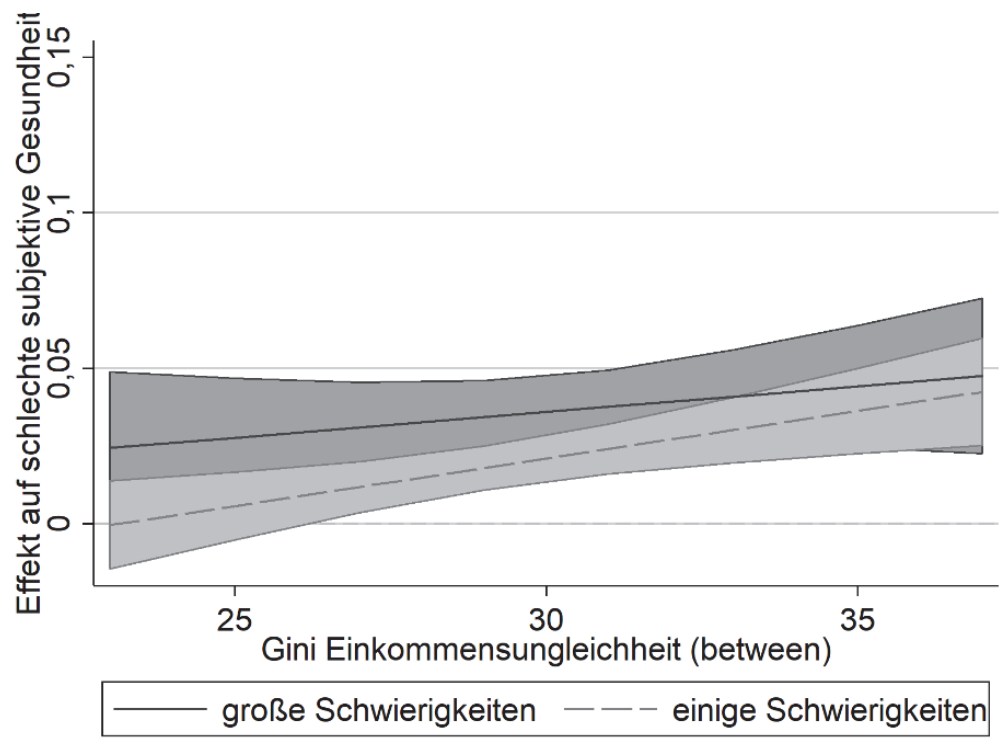

Quelle: SHARE Rel. 6.1.1, Wellen 1, 2, 4, 5, 6. Eigene Berechnungen. Darstellung der Interaktionseffekte und 95\%-Konfidenzintervalle. Die komplette Regressionstabelle befindet sich in Onlineanhang 42. 
Wie in den vorherigen Kapiteln soll auch hier Verschuldung als zweiter Indikator der finanziellen Lage untersucht werden. Dazu werden in weiteren Modellen die Interaktionseffekte von Einkommensungleichheit und dem WithinEffekt von Schulden analysiert (siehe Onlineanhang 44). Bei der Berechnung zur Erklärung von Depression zeigt sich kein signifikanter Interaktionseffekt. Eine größere Ungleichheit im Einkommen erhöht das Depressionsrisiko unabhängig davon, ob man sich verschuldet oder nicht. Hingegen zeigen sich sowohl in den Analysen zu Einschränkungen in den ADL, als auch zur schlechten

Abbildung 7.8: Hybrid-Modell: Effekt von Verschuldung nach Einkommensungleichheit
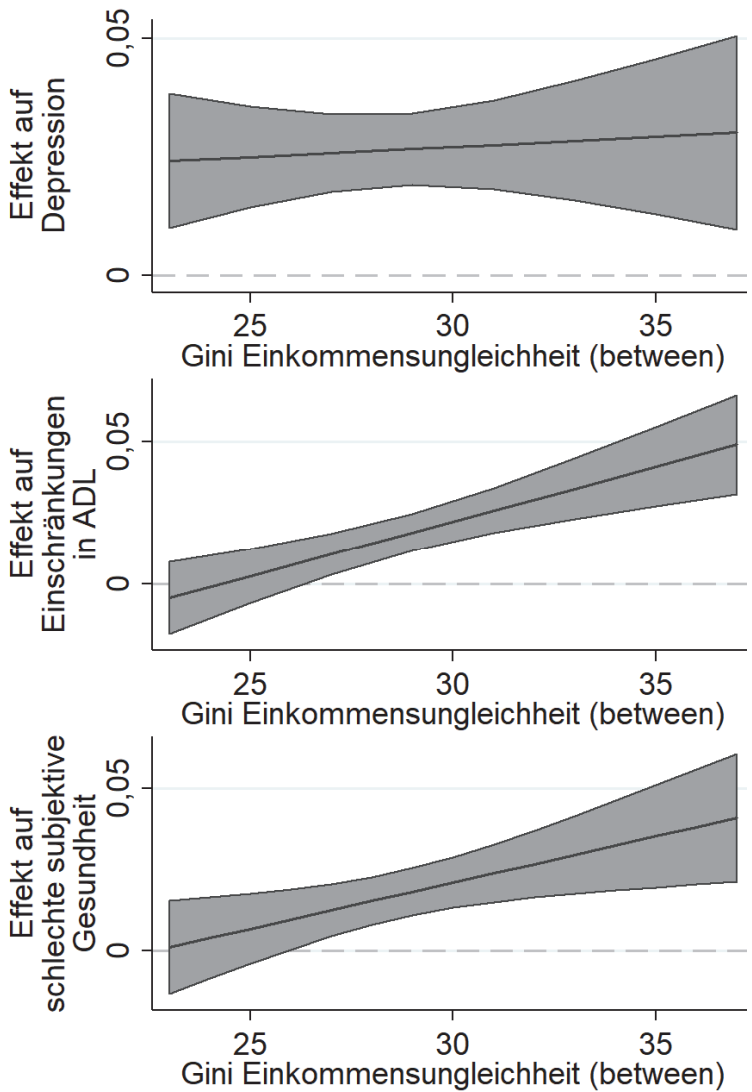

Quelle: SHARE Rel. 6.1.1, Wellen 1, 2, 4, 5, 6. Eigene Berechnungen. Darstellung der Interaktionseffekte und 95\%-Konfidenzintervalle. Die kompletten Regressionstabellen befindet sich in Onlineanhang 44. 
subjektiven Gesundheit signifikante Interaktionseffekte. Eine Verschuldung bei geringer Ungleichheit hat demnach keine negativen gesundheitlichen Folgen. Mit größerer Ungleichverteilung entwickelt sich jedoch ein zunehmender gesundheitsschädlicher Effekt bei Verschuldung. In Abbildung 7.8 sind die Effekte von Verschuldung auf die drei Gesundheitsindikatoren in Abhängigkeit der Einkommensungleichheit dargestellt.

Wie oben beschrieben, korreliert Vermögensungleichheit nicht, oder, im Fall von Depression, entgegen der erwarteten Richtung, mit der Gesundheit. Auch die Aufnahme von Interaktionseffekten (mit der finanziellen Situation und Schulden) in das Modell ändert daran nichts. Bei keinem der drei Gesundheitsindikatoren ist ein signifikanter Interaktionseffekt von Vermögensungleichheit und Veränderung der finanziellen Situation bzw. Verschuldung zu finden.

\subsection{Diskussion der Ergebnisse}

In diesem Kapitel werden die Auswirkungen einer ungleichen Verteilung ökonomischer Ressourcen in einer Gesellschaft auf die Gesundheit der Bevölkerung analysiert. Neben der Vermögens- und Einkommensungleichheit werden dabei auch Indikatoren kontrolliert und getestet, die die neomaterielle und psychosoziale Theorie repräsentieren und den Zusammenhang von Ungleichheit und Gesundheit erklären könnten. Zudem wird untersucht, ob die Folgen für die Gesundheit, die mit einer Veränderung in der individuellen finanziellen Situation einhergehen, auch von dem Ungleichheitsniveau der Gesellschaft beeinflusst werden. Die hier durchgeführte Analyse ist dabei eine der ersten, die die Ungleichheit im Vermögen in diesem Zusammenhang berücksichtigt.

Bei der deskriptiven Betrachtung der Ungleichverteilung ökonomischer Ressourcen kann zunächst festgestellt werden, dass in allen untersuchten Ländern das Vermögen deutlich ungleicher verteilt ist als das Einkommen (vgl. auch Credit Suisse Research Institute 2016). Insgesamt lässt sich in den hier untersuchten Ländern ein schwacher Trend erkennen, dass in Ländern mit höherer Einkommensungleichheit eine niedrigere Vermögensungleichheit besteht. Die genauen Zahlen der Vermögensungleichheit variieren zwar je nach der Datenquelle, dennoch lassen sich Muster erkennen, die auch mit anderen Daten aufgezeigt wurden (Skopek/Buchholz/Blossfeld 2014). Dazu gehört, dass die südeuropäischen Länder eine relativ hohe Ungleichverteilung im Einkommen, jedoch eine geringe Ungleichheit im Vermögen aufweisen. Demgegenüber stehen die skandinavischen Länder, in denen die Ungleichheit im Einkommen gering, im Vermögen hingegen sehr hoch ist. Als Begründungen für diese Muster können verschiedene Faktoren angebracht werden (siehe ebd.: $480 \mathrm{ff}$.). So steht eine geringere Ungleichheit im Vermögen in den südlichen 
Ländern in Zusammenhang mit der Zusammensetzung des Vermögens, insbesondere mit der höheren Quote an Immobilienbesitz (vgl. Kapitel 2.2.8). Für Schweden hingegen kann argumentiert werden, dass das Rentensystem (Garantierente in Kombination mit einer Obergrenze der staatlichen Rentenzahlung) für Geringverdiener keine Anreize bietet Vermögen aufzubauen (Domeij/Klein 2002: 505; Skopek/Buchholz/Blossfeld 2014: 480) und gleichzeitig die Variation im Einkommen verringert. ${ }^{20}$

Betrachtet man die anderen Indikatoren auf Länderebene, so fällt insbesondere der starke Zusammenhang von Wirtschaftskraft (BIP) und sozialem Vertrauen auf. Die Erklärung für den Zusammenhang kann durch kausale Mechanismen in beide Richtungen beschrieben werden (vgl. Bjørnskov 2012). Dass diese Korrelation besteht, wird auch in anderen Untersuchungen bestätigt, allerdings fällt sie bei einer Stichprobe mit mehr Ländern weniger stark aus (Delhey/Newton 2005; Olsen/Dahl 2007).

\subsubsection{Vermögensungleichheit}

Bislang wurde die Vermögensungleichheit nur im Zusammenhang mit Mortalität analysiert (Nowatzki 2012). Die hier vorgestellten Analysen sind die ersten, die mentale Gesundheit, körperliche Einschränkungen und subjektive Gesundheit im Zusammenhang mit der Vermögensungleichheit untersuchen. Bei der Betrachtung der Korrelationen der Vermögensungleichheit mit den drei Gesundheitsindikatoren auf Länderebene im Querschnitt, zeigt sich die Tendenz, dass mit größerer Ungleichheit in einer Gesellschaft eine bessere Gesundheit einhergeht. Während in den multivariaten Berechnungen zu den Einschränkungen in den ADL und subjektiver Gesundheit die Korrelation mit der Vermögensungleichheit verschwindet, bleibt der Zusammenhang bei Depression bestehen. In Ländern mit größerer Ungleichheit im Vermögen ist demnach die Wahrscheinlichkeit an Depression zu leiden geringer. ${ }^{21}$ Die Stärke des berechneten Effektes ist zwar relativ gering, aber dennoch signifikant. Der negative Zusammenhang widerspricht sowohl den theoretischen Argumenten, als auch dem Ergebnis der bislang einzigen Studie zu Vermögensverteilung und Gesundheit. So stellt Nowatzki (2012) fest, dass auch unter Kontrolle verschiedener Indikatoren auf staatlicher Ebene - u.a. Sozialausgaben, Lohnungleichheit, Großzügigkeit des Rentensystems, Wirtschaftskraft - eine geringere Le-

20 Für die hohen Werte der Vermögensungleichheit in Dänemark und Schweden könnten auch methodische Aspekte der Erhebung und Berechnung eine Rolle spielen (Nowatzki 2012: 414).

21 In allen Untersuchungen wurde neben der Vermögens- auch die Einkommensungleichheit kontrolliert. Die Ergebnisse der Vermögensungleichheit bleiben jedoch auch- mit nur geringfügigen Abweichungen -in Modellen, die die Einkommensungleichheit nicht als Kontrollvariable enthalten, bestehen (siehe Onlineanhang 46). 
benserwartung und eine höhere Säuglingssterblichkeit mit größerer Ungleichheit im Vermögen verbunden sind. Neben den abweichenden Gesundheitsindikatoren unterscheidet sich diese Studie von den hier präsentierten Analysen jedoch auch anhand der untersuchten Länder. Nowatzki (2012) untersuchte neun europäische Länder sowie die USA, Kanada, Australien, Neuseeland und Japan. Zudem wurden die Analysen auf Länderebene und nicht anhand von Individualdaten durchgeführt. Ein weiterer methodischer Unterschied besteht in der Auswahl der Kontrollvariablen.

Die in der vorliegenden Studie verwendeten Daten der Vermögensungleichheit können in zweierlei Hinsicht kritisiert werden, was das Ergebnis der Analysen jedoch kaum beeinflussen sollte. Erstens sind keine Daten im Zeitverlauf verfügbar. Dies ist kein spezielles Problem der hier verwendeten Daten, sondern ein generelles Problem, welches auf die Schwierigkeiten der (einheitlichen) Erhebung des Vermögens und das erst kürzlich auftretende Interesse an dem Thema zurückzuführen ist (vgl. Skopek/Buchholz/Blossfeld 2014). In den präsentierten Analysen konnte daher nur ein Koeffizient für das Niveau der Ungleichheit, aber nicht für die Veränderung, berechnet werden. Da das Vermögen im Zeitverlauf jedoch deutlich stabiler ist als das Einkommen (vgl. Nowatzki 2012: 404), ist bei der Verteilung des Vermögens nur eine kleine Variation über die Zeit zu erwarten. Der zweite Kritikpunkt besteht in der Imputation der Gini-Koeffizienten für die Vermögensungleichheit für Estland, Israel, Polen und Tschechien (siehe Kapitel 4.3.2). Allerdings ändern sich die Ergebnisse nur minimal, wenn in den Berechnungen eine Dummyvariable für die Länder mit imputierten Werten berücksichtigt wird. Weitere alternative Analysen werden unter Ausschluss dieser Länder durchgeführt, wobei sich die Varianz in der Vermögensungleichheit verringert, da nur 12 Länder übrig bleiben und die Anzahl der Beobachtungen von 155.520 auf 119.707 sinkt. Die Analysen kommen für Depression und Einschränkungen in den ADL zu denselben Ergebnissen (siehe Onlineanhang 45). Bei der subjektiven Gesundheit zeigen sich jedoch deutliche Unterschiede. Der Koeffizient der Vermögensungleichheit ist unter Ausschluss der vier Länder positiv und signifikant. Zudem dreht sich der Effekt des Niveaus der Einkommensungleichheit um. Die dargestellten Ergebnisse sind also nur belastbar bezüglich der Depression und den Einschränkungen in den ADL. Die Berechnungen zur subjektiven Gesundheit sind anfällig für Verzerrungen, wobei hier nicht festgestellt werden kann, ob dies an der geringeren Fallzahl, den Merkmalen der einzelnen Länder oder den imputierten Werten der Vermögensungleichheit liegt. Insgesamt ist der Effekt der Vermögensungleichheit anfällig für abweichende Ergebnisse bei Ausschluss einzelner Länder. Am stärksten ist dies bei der subjektiven Gesundheit $\mathrm{zu}$ beobachten. Für Einschränkungen in den ADL können geringere und für Depression kaum Abweichungen beobachtet werden. 
Die Forschung zu gesundheitlichen Auswirkungen der Vermögensungleichheit steht noch ganz am Anfang. Die hier präsentierten Analysen beziehen sich nur auf europäische Länder und Israel und zudem auf eine spezielle Bevölkerungsgruppe, Personen ab 50 Jahren. Dennoch liefern sie einen Beitrag zur Erkundung und Etablierung dieses Forschungsfeldes. Weitere Analysen, die sowohl die Verteilung des Vermögens, als auch des Einkommens beleuchten sind nötig, um die Ergebnisse besser einordnen zu können.

\subsubsection{Einkommensungleichheit}

Was die Einkommensungleichheit betrifft, so existiert eine viel größere, fast unübersichtliche, Forschungslandschaft. Das Ergebnis vieler Studien wird auch für die europäische Bevölkerung ab 50 Jahren bestätigt: In Ländern mit größerer Ungleichheit im Einkommen ist die Gesundheit der Bevölkerung schlechter als in Ländern mit geringerer Ungleichheit. Besonders Depression und subjektive Gesundheit zeigen schon in den Querschnittsanalysen auf Länderebene einen deutlichen Zusammenhang mit der Verteilung des Einkommens. Für diese beiden Gesundheitsindikatoren bestätigt sich dies auch in den multivariaten Berechnungen (Between-Effekt). Die Analysen zu Einschränkungen in den ADL zeigen hingegen unter Kontrolle anderer Faktoren eine umgekehrte Korrelation auf. Dieses Ergebnis ist zwar der Theorie entgegengesetzt, allerdings fügt es sich in den uneinheitlichen Forschungsstand zu körperlichen Einschränkungen ein (vgl. Karlsson et al. 2010).

Der zeitverzögerte Zusammenhang von Einkommensungleichheit und Gesundheit konnte aufgrund der beschränkten Datenlage nur in bivariaten Analysen auf Länderebene betrachtet werden. Sie zeigen, dass die Gesundheit der Bevölkerung ab 50 Jahren im Jahr 2015 stärker mit der früheren als mit der aktuellen Ungleichheit korreliert. Für alle drei Gesundheitsindikatoren fällt der Zusammenhang mit der Ungleichheit ab einem Abstand der Messungen von 10 Jahren deutlich stärker aus. Dies spricht dafür, dass die Ungleichheit einer Gesellschaft dauerhaft und mit langfristigen Folgen auf die Bevölkerung einwirkt. Die Ergebnisse stehen im Einklang mit den bisherigen Studien zu dieser Frage (Kondo et al. 2012). In einer Untersuchung wurde die stärkste Korrelation von Ungleichheit und Einschränkungen in der Mobilität bei einem Abstand der Messungen von 20 Jahren gefunden (Gadalla/Fuller-Thomson 2008). Dies kann hier aufgrund der Datenlage nicht getestet werden, aber bei bis zu 15 Jahren Abstand zeigt sich, dass mit zunehmendem Abstand zwischen den Messungen die Korrelation von Ungleichheit und Einschränkungen in den ADL stärker wird. Auch für die subjektive Gesundheit liegt der stärkste Zusammenhang bei 15 Jahren Abstand und entspricht somit den Ergebnissen bisheriger Studien (Blakely 2000; Subramanian/Kawachi 2004). Depression 
korreliert am stärksten mit der Einkommensungleichheit, wenn diese 10 Jahre zuvor gemessen wurde.

Neue Erkenntnisse in einem bislang wenig erforschten Gebiet liefern die Berechnungen bezüglich der gesundheitlichen Auswirkungen von Veränderungen der Ungleichheit. Eine bestmögliche kausale Interpretation wird durch die Verwendung von Individualdaten und die Berechnung der Within-Schätzer möglich. Bisher basierten Längsschnittanalysen nur auf Makrodaten $(\mathrm{Hu} / \mathrm{van}$ Lenthe/Mackenbach 2015) oder auf Daten aus einzelnen Ländern (Bechtel/Lordan/Rao 2012; Lorgelly/Lindley 2008). In der vorliegenden Studie können signifikante Ergebnisse für Depression und subjektive Gesundheit beobachtet werden. Nimmt die Ungleichheit in einem Land im Zeitverlauf zu, so erhöht sich für die Bevölkerung ab 50 Jahren die Wahrscheinlichkeit von Depression und schlechter subjektiver Gesundheit. Das Ergebnis stimmt mit den meisten Ergebnissen bisheriger Studien zu Depression überein (Patel et al. 2018). Im Gegensatz zu der Studie von Vries/Blane/Netuveli (2014), zeigt sich in der vorliegenden Untersuchung jedoch für Einschränkungen in den ADL kein Zusammenhang mit der Veränderung der Einkommensungleichheit. Dies könnte damit zusammenhängen, dass körperliche Einschränkungen nicht als sofortige Reaktion auf Veränderungen der Umwelt entstehen, sondern eine längere Latenzzeit haben (vgl. Kapitel 5.5). Diese Erklärung wird gestützt durch die Analysen mit zeitverzögerter Messung der Ungleichheit. Bei den Einschränkungen in den ADL ist die Korrelation im Falle einer gleichzeitigen Messung deutlich schwächer als bei den beiden anderen Gesundheitsmaßen, wird mit zunehmendem zeitlichem Abstand jedoch immer stärker.

Eine Frage, die sich bei der Analyse (der Veränderung) der Einkommensungleichheit stellt, ist, ob und wie die Mitglieder einer Gesellschaft überhaupt die Verteilung des Einkommens und die relativ geringen Veränderungen im Zeitverlauf wahrnehmen. Studien zeigen, dass die Wirklichkeit und die Wahrnehmung von Ungleichheit in einem Land zum Teil stark voneinander abweichen. Thomas et al. (2018) beschreiben die Wahrnehmung einer stetig wachsenden Ungleichheit in Deutschland und Österreich, selbst wenn dies nicht den Fakten entspricht. Auch wird in Ländern mit ähnlichen (Einkommens-)Ungleichheiten die Ungleichheit teilweise sehr unterschiedlich wahrgenommen (Niehues 2016), was unter anderem mit der Medienpräsenz des Themas zusammenhängen kann (Thomas et al. 2018). Weichen wirkliche und gefühlte Ungleichheit unsystematisch voneinander ab, so ist die subjektive Empfindung der Ungleichheit möglicherweise ein besser geeigneter Indikator für empirische Analysen, insbesondere zur Veränderung der Ungleichheit. 


\subsubsection{Theorievergleich}

Um den Zusammenhang von Ungleichheit und Gesundheit zu erklären wurden zwei Theorien vorgestellt und jeweils anhand eines Indikators in den Analysen berücksichtigt. Der Mechanismus der psychosozialen Theorie wird durch soziales Vertrauen abgebildet. In der Querschnittsbetrachtung lassen sich deutliche Zusammenhänge des sozialen Vertrauens mit den Gesundheitsindikatoren erkennen. Je größer das durchschnittliche soziale Vertrauen in einem Land ist, desto gesünder ist dessen Bevölkerung ab 50 Jahren. Unter Verwendung von Individualdaten und multivariaten Längsschnittmethoden lässt sich dieser $\mathrm{Zu}$ sammenhang jedoch nur für Depression nachweisen.

Der Anteil der privaten Gesundheitsausgaben an den gesamten Gesundheitsausgaben eines Landes dient als Indikator für die neomaterielle Theorie. Anhand dieses Indikators lassen sich in der Querschnittsanalyse auf Länderebene nur schwache Korrelationen mit der Gesundheit der Bevölkerung nachweisen. Es gibt in diesen ersten Analysen einen leichten Trend: Die Bevölkerung ab 50 Jahren ist in den Ländern gesünder, in denen der Staat mehr Gesundheitskosten übernimmt. Unter Kontrolle weiterer Faktoren weisen die Berechnungen jedoch auf keinen oder einen umgekehrten Zusammenhang hin. Dass höhere staatliche Gesundheitsausgaben nicht mit besserer Gesundheit der Bevölkerung einhergehen, wurde auch in anderen Studien gezeigt (Castilla 2004; Olsen/Dahl 2007). Teilweise wurde jedoch das Gegenteil beobachtet (Bergqvist/Yngwe/Lundberg 2013; Adeline/Delattre 2017). Der Großteil der bisherigen Studien verwendet jedoch als Indikator den Anteil der Gesundheitsausgaben am BIP. Eine mögliche Erklärung dafür, dass höhere staatliche Gesundheitsausgaben mit schlechterer Gesundheit einhergehen, ist, dass ein schlechter Gesundheitszustand der Bevölkerung höhere Ausgaben notwendig macht. Möglicherweise spielt auch die Auswahl der untersuchten Länder eine Rolle, die alle - global betrachtet - relativ hohe Gesundheitsausgaben haben und zusätzliche Ausgaben somit eventuell kaum ins Gewicht fallen (Olsen/Dahl 2007).

Um zu testen ob das soziale Vertrauen und der Anteil privater Gesundheitsausgaben den Zusammenhang von Ungleichheit und Gesundheit beeinflussen, werden die Berechnungen mit und ohne Kontrolle der beiden Indikatoren durchgeführt. Die Analysen lassen weder eine Bestätigung der psychosozialen, noch der neomateriellen Theorie, zu. Es gibt keine Hinweise darauf, dass einer der beiden Indikatoren den Zusammenhang von Ungleichheit und Gesundheit vermittelt. Dies widerspricht sowohl den Studien von Elgar (2010) und Layte (2012), die beide die psychosoziale Theorie stützen, als auch Anderson/Bjorklund/Rambotti (2017) sowie Olsen/Dahl (2007), die die Rolle staatlicher Investitionen bekräftigen.

Insgesamt ist der Forschungsstand bei dieser Frage uneinheitlich. Die Gründe für abweichende Ergebnisse können vielfältig sein, z.B. Unterschiede 
hinsichtlich der verwendeten Indikatoren, der untersuchten Länder, der geografischen bzw. politischen Einheiten der Ungleichheitsmessung, der Größe der Stichprobe, der Analysemethode sowie der Auswahl der Kontrollvariablen. Weitere Studien, die beide Theorien direkt miteinander vergleichen sind daher notwendig um ein genaueres Bild der Mechanismen hinter dem Zusammenhang von Ungleichheit und Gesundheit zu erhalten. Dabei sollten jedoch nicht nur bivariate Korrelationen auf Makroebene betrachtet werden, sondern verstärkt Individualdaten herangezogen werden. Die gezeigten Analysen veranschaulichen, dass je nach Methode unterschiedliche Schlüsse gezogen werden können.

\subsubsection{Interaktion: Wer leidet unter der Ungleichheit?}

Ein viel diskutiertes Thema in der Forschung zu den gesundheitlichen Folgen ökonomischer Ungleichheiten ist, ob bestimmte Gruppen der Bevölkerung stärker unter der Ungleichverteilung des Einkommens leiden als der Rest der Gesellschaft. Diese Frage wird hier zum ersten Mal anhand von Längsschnittdaten untersucht. Die Analysen kommen zu relativ einheitlichen Ergebnissen: Je größer die Ungleichheit im Einkommen in einer Gesellschaft ist, desto stärker sind die gesundheitsschädlichen Effekte beim Auftreten von finanziellen Schwierigkeiten. Für Einschränkungen in ADL und subjektiver Gesundheit kann dies auch für Verschuldung gezeigt werden. Wie oben beschrieben zeigt sich in den Berechnungen ein negativer Zusammenhang von Einkommensungleichheit und Einschränkungen in den ADL. In den Modellen mit Interaktionseffekt führt dies dazu, dass Personen bei einer Verbesserung der finanziellen Situation von größerer Ungleichheit profitieren (vgl. Mansyur et al. 2008), während die Effekte eines Abrutschens in große finanzielle Schwierigkeiten bzw. Verschuldung mit größerer Ungleichheit nur leicht ansteigen (siehe Onlineanhang 43).

Der Zusammenfassung von Wilkinson und Pickett (2008; 2009b), dass alle von größerer Gleichheit profitieren, aber die Armen etwas mehr als die Reichen, kann durch die Betrachtung dieser Frage im Längsschnitt ein weiterer Aspekt hinzugefügt werden: Größere und wachsende Ungleichheit ist für alle Statusgruppen einer Gesellschaft mit einer höheren Wahrscheinlichkeit von Depression und schlechter subjektiver Gesundheit verknüpft, wobei vor allem diejenigen darunter leiden, deren finanzielle Situation sich verschlechtert.

Die Analysen mit den Interaktionseffekten deuten auf ein Ergebnis hin, das eine fragwürdige Interpretation zulässt. Die grafischen Darstellungen verdeutlichen, dass - den Berechnungen zufolge - auftretende finanzielle Probleme bei einer geringen Einkommensungleichheit keinen signifikanten Zusammenhang mit der Gesundheit aufweisen. Dies ist jedoch nur am ganz unteren Rand der empirisch untersuchten Spannweite der Einkommensungleichheit der Fall, 
wovon die meisten Länder weit entfernt sind. Zumal das Zustandekommen dieses Befundes auch der linearen Modellierung der Effekte geschuldet sein könnte. Dieses Ergebnis sollte daher nicht als Argument verstanden werden, die Verringerung der Einkommensungleichheit als vorrangiges Ziel der Politik auszugeben und sich weniger um die Bekämpfung individueller Armut zu kümmern. Der Vergleich der Effektstärken macht nämlich deutlich, dass die individuelle finanzielle Situation einen viel stärkeren Einfluss auf die Gesundheit hat als die Einkommensverteilung in der Gesellschaft (vgl. Onlineanhang 42).

\subsubsection{Methodische Diskussion}

Zum Abschluss noch einige methodische Anmerkungen. Das hier verwendete Verfahren, das Hybrid-Modell, fand in der bisherigen Forschung kaum Anwendung (Giesselmann/Windzio 2014). Für die hier untersuchte Forschungsfrage und die vorliegende Datenlage bietet sich dieses Modell an, da sowohl die Vermögensungleichheit (zeitkonstante Variable) als auch die Einkommensungleichheit (zeitveränderliche Variable) untersucht werden kann. Die Berechnung von jeweils Within- und Between-Schätzern für die zeitveränderlichen Variablen ist ein weiterer Vorteil, der auch in der Interaktion ausgenutzt wird. Die Methode bietet jedoch nicht nur Vorteile, weshalb die dargestellten Ergebnisse mit einer gewissen Vorsicht zu betrachten sind. Zum einen sollten Between-Schätzer nicht kausal interpretiert werden, da sie nur auf der Between-Variation beruhen (Brüderl/Ludwig 2015). Zum anderen können die Between-Schätzer durch unbeobachtete Heterogenität verzerrt sein (siehe Diskussion in Kapitel 4.6.5).

Die Between-Schätzer von Einkommensungleichheit, sozialem Vertrauen und dem Anteil der privaten Gesundheitsausgaben wurden wie auch der Schätzer der Vermögensungleichheit als Niveau-Effekte der Länder interpretiert, obwohl zwischen diesen Variablen ein grundlegender Unterschied besteht. Denn im Vergleich zur Vermögensungleichheit, die für jede Beobachtung aus demselben Land gleich ist, basieren die Between-Schätzer der anderen drei Indikatoren auf dem personenspezifischen Mittelwert. ${ }^{22}$ Dieser hängt neben dem Land, in dem die Befragung durchgeführt wurde, auch von den Umfragewellen bzw. den Jahren ab, in denen der oder die Befragte an SHARE teilgenommen hat. Daher wird in alternativen Berechnungen das Hybrid-Modell anstatt mit den Within- und Between-Schätzern der Makroindikatoren mit den konstanten durchschnittlichen Werten (über den gesamten Zeitraum der SHARE Daten, 2004-2015) berechnet. Diese Analysen zeigen, dass die Between-Schätzer der

22 Anhand der Formel (8) auf Seite 116 beschrieben: Vermögensungleichheit ist $z$, das Niveau der Einkommensungleichheit ist $\bar{x}$. 
zeitveränderlichen Variable und die Schätzer der konstanten Durchschnittsvariable nur sehr geringe Abweichungen aufzeigen (siehe Onlineanhang 48). Das gilt auch für die Berechnungen mit den Interaktionen von finanzieller Situation und Einkommensungleichheit. 


\section{Fazit}

Gesundheitliche Ungleichheiten zwischen hohen und niedrigen Statusgruppen innerhalb einer Gesellschaft sind ein bekanntes Phänomen. Die vorliegende Studie erweitert die Forschungslandschaft zu diesem Thema anhand von Analysen der älteren europäischen Bevölkerung. Dazu werden Längsschnittdaten von SHARE aus insgesamt 18 Ländern mit unterschiedlichen Methoden analysiert, um den Einfluss von Armut - hier gemessen anhand von selbstberichteten finanziellen Schwierigkeiten - auf drei Dimensionen der Gesundheit zu untersuchen. Die Ergebnisse ergänzen den bisherigen Forschungsstand bezüglich mehrerer Aspekte: Neben Depression und subjektiver Gesundheit, kommt mit den Einschränkungen in Aktivitäten des täglichen Lebens ein Gesundheitsmaß zum Einsatz, das bisher relativ wenig Aufmerksamkeit bekam, für ältere Menschen jedoch von großer Bedeutung ist. Der Vergleich von Quer- und Längsschnittberechnungen macht deutlich, dass unbeobachtete Heterogenität in Querschnittberechnungen zu verzerrten Ergebnissen führt und Längsschnittdaten und -modelle nötig sind, um kausale Effekte aufzudecken. Im Längsschnitt sind die Korrelationen deutlich kleiner als im Querschnitt. Dennoch ist festzustellen, dass im höheren Alter Veränderungen in der finanziellen Lage einen Einfluss auf die Entwicklung der Gesundheit haben. Ein zentrales Ergebnis der vorliegenden Studie ist, dass die mentale und physische Gesundheit umso stärker leidet, je größer die finanziellen Schwierigkeiten werden. Eine weitere wichtige Erkenntnis ist, dass auch im Alter eine Verbesserung der gesundheitlichen Lage möglich ist. Den Analysen zufolge entspricht (mit vereinzelten Ausnahmen) der negative Effekt einer Verschlechterung in etwa dem positiven Effekt einer Verbesserung der finanziellen Lage. Dies zeigt sich für alle untersuchten Dimensionen von Gesundheit und wurde erstmals speziell für Menschen in höherem Alter untersucht.

Dass auch Veränderungen der gesellschaftlichen ökonomischen Verhältnisse mit Veränderungen der Gesundheit im Alter in Verbindung stehen, ist ein weiteres Ergebnis der vorliegenden Untersuchung. In Bezug auf die subjektive Gesundheit und Depression werden bekannte Ergebnisse bestätigt: Ein höheres $\mathrm{Ma} ß$ an Ungleichverteilung des Einkommens innerhalb einer Gesellschaft ist mit einer höheren Wahrscheinlichkeit schlechter individueller Gesundheit verknüpft. Eine Erweiterung des bisherigen Forschungsstandes liefern die Längsschnittanalysen, die aufzeigen, dass auch Veränderungen der Einkommensverteilung mit gesundheitlichen Indikatoren korrelieren. Erstmals wird in den Analysen betrachtet, wie sich Änderungen der finanziellen Lage unter verschiedenen Einkommensverteilungen auf die Gesundheit auswirken. Den Ergebnissen zufolge spielt das Ausmaß der Ungleichverteilung des Einkommens eine wichtige Rolle für den Zusammenhang von finanziellen und 
gesundheitlichen Veränderungen. Während sich bei geringer Einkommensungleichheit beim Auftreten von finanziellen Problemen keine oder nur geringe Effekte auf die Gesundheit zeigen, steigen die gesundheitsschädlichen Effekte mit zunehmender Einkommensungleichheit an. Demnach sind finanzielle Notlagen umso stärker belastend, je größer die gesellschaftlichen Einkommensunterschiede ausgeprägt sind. Über welche Mechanismen der Zusammenhang von Einkommensverteilung und Gesundheit vermittelt wird, ist noch immer unklar. In den Analysen auf der Makroebene lassen sich teilweise starke $\mathrm{Zu}-$ sammenhänge beobachten, die den beiden gängigen Theorien - psychosoziale und neomaterielle Theorie - entsprechen. Mit den Individualdaten werden jedoch in dieser Arbeit keine Hinweise auf eine Bestätigung der Theorien gefunden. Erstmals wird mit dieser Untersuchung der Zusammenhang von subjektiver Gesundheit, Depression und körperlichen Einschränkungen mit der Vermögensungleichheit beleuchtet. Im Gegensatz zur Einkommensungleichheit wird der erwartete Zusammenhang - schlechtere Gesundheit bei höherer Ungleichheit - mit der Vermögensungleichheit durch die Analysen nicht belegt.

Der Überblick über die Ergebnisse macht auch deutlich, welche Fragen nicht beantwortet werden konnten. Zunächst ist hier der kausale Mechanismus in der Korrelation zwischen finanzieller Lage und Gesundheit zu nennen. Zwar werden in den Analysen die Einflussgrößen der Gesundheit so gut wie möglich kontrolliert und es wird der Versuch unternommen einen Mechanismus der umgekehrten Kausalität auszuschließen (durch die ausschließliche Betrachtung von Personen im Ruhestand kann Krankheit als Ursache für den Verlust der Arbeit und darauf folgende finanzielle Schwierigkeiten ausgeschlossen werden). Ein anderer bedeutender Faktor - die Kosten für die Gesundheitsversorgung - kann in den Berechnungen jedoch nicht berücksichtigt werden, da die Informationen nicht für alle Beobachtungszeitpunkte vorliegen. Die Ergebnisse der Längsschnittanalysen deuten jedoch darauf hin, dass sich die finanzielle Situation kausal auf die Gesundheit auswirkt. Eine weitere offene Frage betrifft den Mechanismus hinter der Korrelation von Einkommensverteilung und Gesundheit. Die inkonsistenten Ergebnisse machen deutlich, dass weitere Untersuchungen nötig sind, um die Gültigkeit der neomateriellen und psychosozialen Theorie zu testen. Bei der Interpretation der Ergebnisse zur Vermögensungleichheit ist aus mehreren Gründen Vorsicht geboten: Die Qualität der Datenbasis ist nicht optimal und von Land zu Land unterschiedlich, die Anzahl der untersuchten Länder ist gering und die Ergebnisse sind davon abhängig, welche Länder untersucht werden. Der Zusammenhang von Vermögensungleichheit und Gesundheit sollte in weiteren Studien mit verschiedenen Gesundheitsindikatoren und mehr Ländern untersucht werden, wobei dies durch die großen Lücken in der Datenlage bezüglich der Vermögensungleichheiten momentan schwierig ist. Insgesamt ist die Verfügbarkeit von geeigneten Daten eine wichtige Voraussetzung für weitergehende, zukünftige Forschung. Um Veränderungen der Gesundheit im Alter genauer zu untersuchen und mögliche 
Anpassungen an neue Situationen aufzuzeigen, ist eine Datenbasis mit längerer Beobachtungsdauer nötig.

Für die Entstehung von mentalen und physischen Krankheiten gibt es eine Vielzahl an möglichen Einflussfaktoren. Die vorliegende Untersuchung zeigt, welche Rolle die individuelle und gesellschaftliche ökonomische Situation dabei spielt. Es wird deutlich, dass finanzielle Notlagen nicht nur die Kaufkraft und den Konsum einschränken, sie können auch die mentale und physische Gesundheit negativ beeinflussen. Um gesundheitliche Ungleichheiten in einer Gesellschaft zu verringern, gibt es viele mögliche Ansatzpunkte. Natürlich sollte die Verhinderung von finanziellen Notlagen ein wichtiger Punkt der Politikgestaltung sein. Da sich der soziale Status und die Armut über das ganze Leben entwickeln, ist hierbei zum einen der gesamte Lebenslauf zu berücksichtigen, z.B. anhand der Bildungschancen (BMAS 2017). Zum anderen können Veränderungen im Leben der älteren Menschen für die finanzielle Situation (und Gesundheit) von Bedeutung sein, was Hilfs- und Präventionsprogramme begleitend $\mathrm{zu}$ verschiedenen Lebensereignissen - z.B. Verwitwung und Übergang in den Ruhestand - nahelegt. Die Analysen zeigen, dass die Hilfe zur Überwindung von finanziellen Problemen ein möglicher Ansatzpunkt zur Verbesserung von Phasen schlechter Gesundheit darstellt. Da die genauen Mechanismen hinter dem Zusammenhang von Einkommensungleichheit und Gesundheit noch immer unklar sind, ist es schwierig diesbezüglich gezielte Maßnahmen zu entwickeln. Um eine Verringerung der Ungleichheit im Einkommen zu erreichen, können z.B. steuerpolitische Maßnahmen, wie eine Anpassung der Einkommenssteuer, oder auf den Arbeitsmarkt bezogene, zur Verhinderung von Arbeitslosigkeit ausgerichtete, Maßnahmen angewendet werden (BMF 2017). Für die ältere Bevölkerung sind hauptsächlich rentenpolitische Maßnahmen relevant. 



\section{Literaturverzeichnis}

Acciai, Francesco/Hardy, Melissa (2017): Depression in later life: A closer look at the gender gap. In: Social science research 68, S. 163-175.

Adeline, Amélie/Delattre, Eric (2017): Some microeconometric evidence on the relationship between health and income. In: Health economics review 7, 1, S. 27.

Adena, Maja/Myck, Michal (2014): Poverty and transitions in health in later life. In: Social science \& medicine 116, S. 202-210.

Adena, Maja/Myck, Michał/Oczkowska, Monika (2015): Material deprivation items in SHARE Wave 5 data: a contribution to a better understanding of differences in material conditions in later life. In: Börsch-Supan, A./Kneip, T./Litwin, H./Myck, M./Weber, G. (Hrsg.): Ageing in Europe - Supporting Policies for an Inclusive Society. Berlin, München, Boston: DE GRUYTER, S. 25-38.

Adler, Nancy E./Epel, Elissa S./Castellazzo, Grace/Ickovics, Jeannette R. (2000): Relationship of subjective and objective social status with psychological and physiological functioning. Preliminary data in healthy, White women. In: Health Psychology 19, 6, S. 586-592.

Ahnquist, Johanna/Fredlund, Peeter/Wamala, Sarah P. (2007): Is cumulative exposure to economic hardships more hazardous to women's health than men's? A 16-year follow-up study of the Swedish Survey of Living Conditions. In: Journal of epidemiology and community health 61, 4, S. 331-336.

Ahnquist, Johanna/Wamala, Sarah P./Lindstrom, Martin (2012): Social determinants of health--a question of social or economic capital? Interaction effects of socioeconomic factors on health outcomes. In: Social science \& medicine (1982) 74, 6 , S. 930-939.

Ai, Chunrong/Norton, Edward C. (2003): Interaction terms in logit and probit models. In: Economics Letters 80, 1, S. 123-129.

Aida, Jun/Kondo, Katsunori/Kondo, Naoki/Watt, Richard G./Sheiham, Aubrey/Tsakos, Georgios (2011): Income inequality, social capital and self-rated health and dental status in older Japanese. In: Social science \& medicine (1982) 73, 10, S. 15611568.

Aittomäki, Akseli/Martikainen, Pekka/Laaksonen, Mikko/Lahelma, Eero/Rahkonen, Ossi (2010): The associations of household wealth and income with self-rated health--a study on economic advantage in middle-aged Finnish men and women. In: Social science \& medicine (1982) 71, 5, S. 1018-1026.

Alcock, Pete (1993): Understanding poverty. Basingstoke: Macmillan.

Alesina, Alberto/La Ferrara, Eliana (2000): Participation in Heterogeneous Communities. In: Quarterly Journal of Economics 115, 3, S. 847-904.

Allison, Paul D. (2009): Fixed Effects Regression Models. Thousand Oaks: Sage.

Amann, Gabriele (1991): Die Relevanz der Dauer einer Depression für das soziale Netzwerk und die soziale Unterstützung. In: Der Nervenarzt, 62, S. 557-564.

Andersen, Christian K./Wittrup-Jensen, Kim U./Lolk, Anette/Andersen, Kjeld/KraghSørensen, Per (2004): Ability to perform activities of daily living is the main factor affecting quality of life in patients with dementia. In: Health and quality of life outcomes 2, S. 52. 
Andersen-Ranberg, K./Christensen, Kaare/Jeune, Bernard/Skytthe, Axel/Vasegaard, Lone/Vaupel, James W. (1999): Declining physical abilities with age. A cross-sectional study of older twins and centenarians in Denmark. In: Age and Ageing 28, 4, S. 373-377.

Andersen-Ranberg, Karen/Petersen, I./Frederiksen, H./Mackenbach, J. P./Christensen, K. (2009): Cross-national differences in grip strength among 50+ year-old Europeans: results from the SHARE study. In: European journal of ageing 6, 3, S. $227-$ 236.

Andersen-Ranberg, Karen/Petersen, Inge/Robine, Jean-Marie/Christensen, Kaare (2005): Who Are the Oldest-Old? In: Börsch-Supan, A./Brugiavini, A./Jürges, H./Mackenbach, J./Siegrist, J./Weber, G. (Hrsg.): Health, aging and retirement in Europe. First results from the Survey of Health, ageing and retirement in Europe. Mannheim: Mannheim Research Institute for the Economics of Aging (MEA), S. $35-40$.

Anderson, Kathryn/Bjorklund, Eric/Rambotti, Simone (2017): Income Inequality and Chronic Health Conditions: A Multilevel Analysis of the U.S. States. SocArXiv.

Anderson, R. J./Freedland, K. E./Clouse, R. E./Lustman, P. J. (2001): The Prevalence of Comorbid Depression in Adults With Diabetes. A meta-analysis. In: Diabetes Care 24, 6, S. 1069-1078.

Andreß, Hans-Jürgen/Golsch, Katrin/Schmidt, Alexander W. (2013): Applied Panel Data Analysis for Economic and Social Surveys. Berlin, Heidelberg: Springer Berlin Heidelberg.

Angel, Ronald J./Frisco, Michelle/Angel, Jacqueline L./Chiriboga, David A. (2003): Financial Strain and Health among Elderly Mexican-Origin Individuals. In: Journal of Health and Social Behavior 44, 4, S. 536.

Angelini, Viola/Howdon, Daniel D. D./Mierau, Jochen O. (2018): Childhood Socioeconomic Status and Late-Adulthood Mental Health: Results from the Survey on Health, Ageing and Retirement in Europe. In: The journals of gerontology. Series B, Psychological sciences and social sciences.

Anisman, Hymie/Merali, Zul/Hayley, Shawn (2008): Neurotransmitter, peptide and cytokine processes in relation to depressive disorder: comorbidity between depression and neurodegenerative disorders. In: Progress in neurobiology 85, 1, S. 1-74.

Anstey, Kaarin J./Sanden, Chwee von/Sargent-Cox, Kerry/Luszcz, Mary A. (2007): Prevalence and risk factors for depression in a longitudinal, population-based study including individuals in the community and residential care. In: The American journal of geriatric psychiatry official journal of the American Association for Geriatric Psychiatry 15, 6, S. 497-505.

Apouey, Benedicte/Clark, Andrew E. (2015): Winning big but feeling no better? The effect of lottery prizes on physical and mental health. In: Health economics 24, 5 , S. 516-538.

Arezzo, Maria Felice (2018): Social Capital, Income Inequality and the Health of the Elderly. In: Skiadas, C./Skiadas, C. (Hrsg.): Demography and Health Issues. Population Aging, Mortality and Data Analysis. The Springer Series on Demographic Methods and Population Analysis, volume 46. Cham: Springer International Publishing, S. 301-313. 
Arezzo, Maria Felice/Giudici, Cristina (2017): Social Capital and Self Perceived Health Among European Older Adults. In: Social Indicators Research 130, 2, S. 665-685.

Aro, Arja R./Avendano, Mauricio/Mackenbach, Johan (2005): Health Behaviour. In: Börsch-Supan, A./Brugiavini, A./Jürges, H./Mackenbach, J./Siegrist, J./Weber, G. (Hrsg.): Health, Ageing and Retirement in Europe - First Results from the Survey of Health, Ageing and Retirement in Europe. Mannheim, S. 102-107.

Auger, Nathalie/Hamel, Denis/Martinez, Jérôme/Ross, Nancy A. (2012): Mitigating effect of immigration on the relation between income inequality and mortality: a prospective study of 2 million Canadians. In: Journal of epidemiology and community health 66,6 , S. e5.

Avendano, Mauricio/Hessel, Philipp (2015): The income inequality hypothesis rejected? In: European journal of epidemiology 30, 8, S. 595-598.

Backholer, K./Wong, E./Freak-Poli, R./Walls, H. L./Peeters, A. (2012): Increasing body weight and risk of limitations in activities of daily living: a systematic review and meta-analysis. In: Obesity reviews an official journal of the International Association for the Study of Obesity 13, 5, S. 456-468.

Backlund, Eric/Sorlie, Paul D./Johnson, Norman J. (1996): The shape of the relationship between income and mortality in the United States. In: Annals of epidemiology 6,1, S. 12-20.

Balmuth, Evan (2017): From Black Bile to the Brain. Tracing Melancholia and Depression. In: The Offspring, 2017, S. 8-10.

Bandura, Albert (1977): Self-efficacy. Toward a unifying theory of behavioral change. In: Psychological Review 84, 2, S. 191-215.

Banks, James/Muriel, Alastair/Smith, James P. (2011): Attrition and health in ageing studies: Evidence from ELSA and HRS. In: Longitudinal and life course studies 2, 2.

Baranzini, Andrea/Ramirez, José V. (2016): Paying for Quietness. The Impact of Noise on Geneva Rents. In: Urban Studies 42, 4, S. 633-646.

Barbato, Angelo/Vallarino, Martine/Rapisarda, Filippo/Lora, Antonio/Caldas de Almeida, José Miguel (2016): Access to mental health care in Europe. https://ec.europa.eu/health/sites/health/files/mental_health/docs/ev_20161006_co02_en.pdf.

Bartley, Mel (2017): Health inequality. An introduction to concepts, theories and methods. Second edition. Cambridge, UK, Malden, MA: Polity Press.

Bartley, Mel/Plewis, Ian (2002): Accumulated labour market disadvantage and limiting long-term illness. Data from the 1971-1991 Office for National Statistics' Longitudinal Study. In: International journal of epidemiology 31, 2, S. 336-341.

Bechtel, Lucy/Lordan, Grace/Rao, D. S. Prasada (2012): Income inequality and mental health-empirical evidence from Australia. In: Health economics 21 Suppl 1, S. 4 17.

Beck, Aaron T. (1987): Cognitive models of depression. In: The Journal of Cognitive Psychotherapy: An International Quarterly 1, 1, S. 5-37.

Becker, Simone/Huy, Christina/Brinkhoff, Klaus-Peter/Gomolinsky, Uwe/Klein, Thomas/Thiel, Ansgar/Zimmermann-Stenzel, Monique (2007): „Ein aktives Leben leben"--Sport, Bewegung und Gesundheit im mittleren und höheren Erwachsenenalter. Konzeption, Datenerhebung und erste Ergebnisse eines repräsentativen 
Basis-Survey für die 50- bis 70-jährige baden-württembergische Wohnbevölkerung. In: Gesundheitswesen (Bundesverband der Ärzte des Offentlichen Gesundheitsdienstes (Germany)) 69, 7, S. 401-407.

Becker, Simone/Schneider, Sven (2005): Analysen zur Sportbeteiligung auf der Basis des repräsentativen Bundes-Gesundheitssurveys 1998. In: Sport und Gesellschaft $2,2$.

Beckfield, Jason (2004): Does income inequality harm health? New cross-national evidence. In: Journal of Health and Social Behavior 45, 3, S. 231-248.

Beekman, Aartjan T. F./Deeg, D.J.H/Geerlings, S.W/Schoevers, R.A/Smit, J.H/van Tilburg, W. (2001): Emergence and persistence of late life depression. A 3-year follow-up of the Longitudinal Aging Study Amsterdam. In: Journal of Affective Disorders 65, 2, S. 131-138.

Benyamini, Yael (2011): Why does self-rated health predict mortality? An update on current knowledge and a research agenda for psychologists. In: Psychology \& health 26, 11, S. 1407-1413.

Berger, Lawrence M./Collins, J. Michael/Cuesta, Laura (2016): Household Debt and Adult Depressive Symptoms in the United States. In: Journal of Family and Economic Issues 37, 1, S. 42-57.

Bergqvist, Kersti/Yngwe, Monica Aberg/Lundberg, Olle (2013): Understanding the role of welfare state characteristics for health and inequalities - an analytical review. In: BMC public health 13, S. 1234.

Berkman, Lisa F./Krishna, Aditi (2014): Social network epidemiology. In: Berkman, L. F./Kawachi, I./Glymour, M. M. (Hrsg.): Social epidemiology. 2 ed. New York: Oxford University Press, S. 234-289.

Bernabé, E./Marcenes, W. (2011): Income inequality and tooth loss in the United States. In: Journal of dental research 90, 6, S. 724-729.

Bertoni, Marco/Maggi, Stefania/Manzato, Enzo/Veronese, Nicola/Weber, Guglielmo (2018): Depressive symptoms and muscle weakness: A two-way relation? In: Experimental gerontology 108, S. 87-91.

Best, Henning/Wolf, Christof (2010): Logistische Regression. In: Wolf, C./Best, H. (Hrsg.): Handbuch der sozialwissenschaftlichen Datenanalyse. Wiesbaden: VS Verlag für Sozialwissenschaften, S. 827-854.

Bhandari, B./Newton, J. T./Bernabé, E. (2015): Income Inequality and Use of Dental Services in 66 Countries. In: Journal of dental research 94, 8, S. 1048-1054.

Biggs, Brian/King, Lawrence/Basu, Sanjay/Stuckler, David (2010): Is wealthier always healthier? The impact of national income level, inequality, and poverty on public health in Latin America. In: Social science \& medicine (1982) 71, 2, S. 266-273.

Bisschop, M. Isabella/Kriegsman, Didi M. W./Beekman, Aartjan T. F./Deeg, Dorly J. H. (2004): Chronic diseases and depression: the modifying role of psychosocial resources. In: Social science \& medicine (1982) 59, 4, S. 721-733.

Bjørnskov, Christian (2012): How Does Social Trust Affect Economic Growth? In: Southern Economic Journal 78, 4, S. 1346-1368.

Blakely, T. A. (2000): What is the lag time between income inequality and health status? In: Journal of Epidemiology \& Community Health 54, 4, S. 318-319. 
Blane, David/Smith, George Davey/Bartley, Mel (1993): Social selection. What does it contribute to social class differences in health? In: Sociology of Health \& Illness 15,1, S. $1-15$.

Blazer, Dan G. 2nd/Hybels, Celia F. (2005): Origins of depression in later life. In: Psychological medicine 35, 9, S. 1241-1252.

Blázquez Cuesta, Maite/Budría, Santiago (2015): Income deprivation and mental wellbeing: the role of non-cognitive skills. In: Economics and human biology 17, S. 16-28.

Bloomfield, Kim/Grittner, Ulrike/Kramer, Stephanie/Gmel, Gerhard (2006): Social inequalities in alcohol consumption and alcohol-related problems in the study countries of the EU concerted action 'Gender, Culture and Alcohol Problems: a Multinational Study'. In: Alcohol and alcoholism (Oxford, Oxfordshire). Supplement 41,1, S. i26-36.

BMAS (2017): Lebenslagen in Deutschland. Der Fünfte Armuts- und Reichtumsbericht der Bundesregierung.

BMF (2017): Einkommensungleichheit und soziale Mobilität. Gutachten des Wissenschaftlichen Beirats beim Bundesministerium der Finanzen. Berlin.

Bock, Joerg/Wainstock, Tamar/Braun, Katharina/Segal, Menahem (2015): Stress In Utero: Prenatal Programming of Brain Plasticity and Cognition. In: Biological psychiatry 78,5 , S. 315-326.

Bolte, Gabriele/Kohlhuber, Martina (2009): Soziale Ungleichheit bei umweltbezogener Gesundheit: Erklärungsansätze aus umweltepidemiologischer Perspektive. In: Richter, M./Hurrelmann, K. (Hrsg.): Gesundheitliche Ungleichheit. Grundlagen, Probleme, Perspektiven. 2., aktualisierte Aufl. Wiesbaden: VS Verlag für Sozialwissenschaften / GWV Fachverlage, Wiesbaden, S. 99-116.

Börsch-Supan, Axel/Brandt, Martina/Hunkler, Christian/Kneip, Thorsten/Korbmacher, Julie/Malter, Frederic/Schaan, Barbara/Stuck, Stephanie/Zuber, Sabrina (2013): Data Resource Profile: the Survey of Health, Ageing and Retirement in Europe (SHARE). In: International journal of epidemiology 42, 4, S. 992-1001.

Börsch-Supan, Axel/Bucher-Koenen, Tabea/Hanemann, Felizia (2017a): Does Disability Insurance Improve Health and Well-Being? MEA Discussion Papers. München.

Börsch-Supan, Axel/Bucher-Koenen, Tabea/Hanemann, Felizia (2017b): Early Determinants of Work Disability in an International Perspective. MEA Discussion Papers. München.

Börsch-Supan, Axel/Gasche, Martin (2010): Zunehmende Altersarmut in Deutschland - vermeidbar oder unvermeidlich? policy brief. Mannheim.

Bosch, Gerhard (2012): Prekäre Beschäftigung und Neuordnung am Arbeitsmarkt. Expertise im Auftrag der Industriegewerkschaft Metall. IAQ-Standpunkt.

Bourcarde, Kay (2012): Ein „Alter ohne Not" - Perspektiven der Alterssicherung in Deutschland. In: Huster, E.-U./Boeckh, J./Mogge-Grotjahn, H. (Hrsg.): Handbuch Armut und Soziale Ausgrenzung. Wiesbaden: VS Verlag für Sozialwissenschaften, S. 453-468.

Bourdieu, Pierre (1987): Die feinen Unterschiede. Kritik der gesellschaftlichen Urteilskraft. Berlin: Suhrkamp. 
Boydell, J./van Os, J./McKenzie, K./Murray, R. M. (2004): The association of inequality with the incidence of schizophrenia--an ecological study. In: Social psychiatry and psychiatric epidemiology 39, 8, S. 597-599.

BPtK (2018): Ein Jahr nach der Reform der Psychotherapie-Richtlinie. Wartezeiten 2018. Bundespsychotherapeutenkammer. Berlin.

Brandt, Martina (2009): Hilfe zwischen Generationen. Ein europäischer Vergleich. Wiesbaden: VS Verl. für Sozialwissenschaften.

Brandt, Martina/Deindl, Christian/Hank, Karsten (2012): Tracing the origins of successful aging: the role of childhood conditions and social inequality in explaining later life health. In: Social science \& medicine (1982) 74, 9, S. 1418-1425.

Brent, David/Melhem, Nadine/Donohoe, M. Bertille/Walker, Monica (2009): The incidence and course of depression in bereaved youth 21 months after the loss of a parent to suicide, accident, or sudden natural death. In: The American Journal of Psychiatry 166, 7, S. 786-794.

Breyer, Bianka (2015): Social Trust Scale (ESS). ZIS - GESIS Leibniz Institute for the Social Sciences.

Bridges, Sarah/Disney, Richard (2010): Debt and depression. In: Journal of health economics 29,3 , S. 388-403.

Bronisch, Thomas (1992): Die depressive Reaktion. Probleme der Klassifikation, Diagnostik und Pathogenese, Band 68. Berlin, Heidelberg: Springer.

Brown, G. W./Andrews, B./Harris, T./Adler, Z./Bridge, L. (1986): Social support, selfesteem and depression. In: Psychological Medicine 16, 04, S. 813.

Brown, G. W./Harris, T. O./Hepworth, C. (1995): Loss, humiliation and entrapment among women developing depression. A patient and non-patient comparison. In: Psychological Medicine 25, 01, S. 7.

Bruce, Martha L. (2001): Depression and Disability in Late Life. Directions for Future Research. In: The American Journal of Geriatric Psychiatry 9, 2, S. 102-112.

Brüderl, Josef (2010): Kausalanalyse mit Paneldaten. In: Wolf, C./Best, H. (Hrsg.): Handbuch der sozialwissenschaftlichen Datenanalyse. Wiesbaden: VS Verlag für Sozialwissenschaften, S. 963-994.

Brüderl, Josef/Ludwig, Volker (2015): Fixed-effects panel regression. In: Best, H./Wolf, C. (Hrsg.): The Sage handbook of regression analysis and causal inference. London: Sage, S. 327-357.

Bruin, A. de/Picavet, H.S.J./Nossikov, A. (1996): Health interview surveys. Towards international harmonization of methods and instruments. WHO regional publications. European series, Band 58. Copenhagen: WHO regional Office for Europe.

Brülle, Jan (2018): Poverty Trends in Germany and Great Britain. Wiesbaden: Springer Fachmedien Wiesbaden.

Brunner, Eric (1997): Socioeconomic determinants of health. Stress and the biology of inequality. In: BMJ, 314, S. 1472-1476.

Buhr, Petra (1995): Dynamik von Armut. Wiesbaden: VS Verlag für Sozialwissenschaften.

Burke, Heather M./Davis, Mary C./Otte, Christian/Mohr, David C. (2005): Depression and cortisol responses to psychological stress: a meta-analysis. In: Psychoneuroendocrinology 30, 9, S. 846-856. 
Burkhauser, Richard V./Hahn, Markus H./Lillard, Dean R./Wilkins, Roger (2016): Does Income Inequality in Early Childhood Predict Self-Reported Health in Adulthood? A Cross-National Comparison of the United States and Great Britain. In: Lorenzo Cappellari/Solomon W Polachek/Konstantinos Tatsiramos (Hrsg.): Inequality. Causes and Consequences. Research in Labor Economics, Band 43. Bingley, U.K.: Emerald, S. 407-476.

Burns, Jonathan K./Esterhuizen, Tonya (2008): Poverty, inequality and the treated incidence of first-episode psychosis: an ecological study from South Africa. In: Social psychiatry and psychiatric epidemiology 43, 4, S. 331-335.

Burns, Jonathan K./Tomita, Andrew/Kapadia, Amy S. (2014): Income inequality and schizophrenia: increased schizophrenia incidence in countries with high levels of income inequality. In: The International journal of social psychiatry 60, 2, S. 185196.

Burzan, Nicole (2011): Soziale Ungleichheit. Eine Einführung in die zentralen Theorien. 4. Aufl. Hagener Studientexte zur Soziologie. Wiesbaden: VS Verlag für Sozialwissenschaften.

Busch, M. A./Maske, U. E./Ryl, L./Schlack, R./Hapke, U. (2013): Prävalenz von depressiver Symptomatik und diagnostizierter Depression bei Erwachsenen in Deutschland: Ergebnisse der Studie zur Gesundheit Erwachsener in Deutschland (DEGS1). In: Bundesgesundheitsblatt, Gesundheitsforschung, Gesundheitsschutz 56, 5-6, S. 733-739.

Butterwegge, Carolin/Hansen, Dirk (2012): Altersarmut ist überwiegend weiblich. Frauen als Hauptleidtragende des Sozialabbaus. In: Butterwegge, C./Bosbach, G./Birkwald, M. W. (Hrsg.): Armut im Alter. Probleme und Perspektiven der sozialen Sicherung. Frankfurt am Main, New York: Campus, S. 111-129.

Butterwegge, Christoph (2018): Armut. In: Kopp, J./Steinbach, A. (Hrsg.): Grundbegriffe der Soziologie. Wiesbaden: Springer Fachmedien Wiesbaden, S. 29-31.

Butterworth, Peter/Rodgers, Bryan/Windsor, Tim D. (2009): Financial hardship, socioeconomic position and depression: results from the PATH Through Life Survey. In: Social science \& medicine 69, 2, S. 229-237.

Cabeza, Elena/Esteva, Magdalena/Pujol, Angels/Thomas, Vicenç/Sánchez-Contador, Carmen (2007): Social disparities in breast and cervical cancer preventive practices. In: European journal of cancer prevention the official journal of the European Cancer Prevention Organisation (ECP) 16, 4, S. 372-379.

Calvó-Perxas, Laia/Vilalta-Franch, Joan/Turró-Garriga, Oriol/López-Pousa, Secundino/Garre-Olmo, Josep (2016): Gender differences in depression and pain: A two year follow-up study of the Survey of Health, Ageing and Retirement in Europe. In: Journal of Affective Disorders 193, S. 157-164.

Carere, Claudio/Welink, Diliana/Drent, Piet J./Koolhaas, Jaap M./Groothuis, Ton G.G (2001): Effect of social defeat in a territorial bird (Parus major) selected for different coping styles. In: Physiology \& Behavior 73, 3, S. 427-433.

Carlson, Andrea/Frazao, Elizabeth (2012): Are Healthy Foods Really More Expensive? It Depends on How You Measure the Price. In: USDA-ERS Economic Information Bulletin 96.

Carr, Deborah/Springer, Kristen W. (2010): Advances in Families and Health Research in the 21 st Century. In: Journal of Marriage and Family 72, 3, S. 743-761. 
Carter, K. N./Blakely, T./Collings, S./Imlach Gunasekara, F./Richardson, K. (2009): What is the association between wealth and mental health? In: Journal of epidemiology and community health 63, 3, S. 221-226.

Castilla, Emilio J. (2004): Organizing Health Care. A Comparative Analysis of National Institutions and Inequality Over Time. In: International Sociology 19, 4, S. 403435.

Celidoni, Martina/Rebba, Vincenzo (2017): Healthier lifestyles after retirement in Europe? Evidence from SHARE. In: The European journal of health economics HEPAC health economics in prevention and care 18, 7, S. 805-830.

Chapman, Benjamin P./Fiscella, Kevin/Kawachi, Ichiro/Duberstein, Paul R. (2010): Personality, socioeconomic status, and all-cause mortality in the United States. In: American journal of epidemiology 171, 1, S. 83-92.

Chatfield, Mark D./Brayne, Carol E./Matthews, Fiona E. (2005): A systematic literature review of attrition between waves in longitudinal studies in the elderly shows a consistent pattern of dropout between differing studies. In: Journal of clinical epidemiology 58, 1, S. 13-19.

Chen, Zhuo/Gotway Crawford, Carol A. (2012): The role of geographic scale in testing the income inequality hypothesis as an explanation of health disparities. In: Social science \& medicine (1982) 75, 6, S. 1022-1031.

Cheng, Cecilia/Cheung, Shu-Fai/Chio, Jasmine Hin-Man/Chan, Man-Pui Sally (2013): Cultural meaning of perceived control: a meta-analysis of locus of control and psychological symptoms across 18 cultural regions. In: Psychological bulletin 139, 1, S. 152-188.

Chiavegatto Filho, Alexandre Dias Porto/Kawachi, Ichiro/Wang, Yuan Pang/Viana, Maria Carmen/Andrade, Laura Helena Silveira Guerra (2013): Does income inequality get under the skin? A multilevel analysis of depression, anxiety and mental disorders in Sao Paulo, Brazil. In: Journal of epidemiology and community health 67, 11, S. 966-972.

Choi, HwaJung/Burgard, Sarah/Elo, Irma T./Heisler, Michele (2015): Are older adults living in more equal counties healthier than older adults living in more unequal counties? A propensity score matching approach. In: Social science \& medicine (1982) 141, S. 82-90.

Christian, Lisa M./Glaser, Ronald/Porter, Kyle/Malarkey, William B./Beversdorf, Da$\mathrm{vid} /$ Kiecolt-Glaser, Janice K. (2011): Poorer self-rated health is associated with elevated inflammatory markers among older adults. In: Psychoneuroendocrinology 36,10, S. 1495-1504.

Chum (2012): Socio-Environmental Determinants of Cardiovascular Diseases. Doctoral dissertation. Toronto: University of Toronto.

Cifuentes, Manuel/Sembajwe, Grace/Tak, SangWoo/Gore, Rebecca/Kriebel, Da$\mathrm{vid} /$ Punnett, Laura (2008): The association of major depressive episodes with income inequality and the human development index. In: Social science $\&$ medicine (1982) 67, 4, S. 529-539.

Clark, Andrew E./Senik, Claudia (2010): Who Compares to Whom? The Anatomy of Income Comparisons in Europe. In: The Economic Journal 120, 544, S. 573-594. 
Clarke, P./Marshall, V./House, J./Lantz, P. (2011): The Social Structuring of Mental Health over the Adult Life Course. Advancing Theory in the Sociology of Aging. In: Social Forces 89, 4, S. 1287-1313.

Clarke, Philippa/Wheaton, Blair (2005): Mapping Social Context on Mental Health Trajectories Through Adulthood. In: Advances in Life Course Research 9, S. 269301.

Clayton, Maya/Liñares-Zegarra, José/Wilson, John O. S. (2015): Does debt affect health? Cross country evidence on the debt-health nexus. In: Social science \& medicine (1982) 130, S. 51-58.

Clough-Gorr, Kerri M./Egger, Matthias/Spoerri, Adrian (2015): A Swiss paradox? Higher income inequality of municipalities is associated with lower mortality in Switzerland. In: European journal of epidemiology 30, 8, S. 627-636.

Coburn, David (2015): Income inequality, welfare, class and health: A comment on Pickett and Wilkinson, 2015. In: Social science \& medicine (1982) 146, S. 228 232.

Coe, Norma B./Zamarro, Gema (2011): Retirement effects on health in Europe. In: Journal of health economics 30, 1, S. 77-86.

Cohen, Sheldon/Alper, Cuneyt M./Doyle, William J./Adler, Nancy/Treanor, John J./Turner, Ronald B. (2008): Objective and subjective socioeconomic status and susceptibility to the common cold. In: Health psychology official journal of the Division of Health Psychology, American Psychological Association 27, 2, S. 268-274.

Connolly, Deirdre/Garvey, Jess/McKee, Gabrielle (2017): Factors associated with ADL/IADL disability in community dwelling older adults in the Irish longitudinal study on ageing (TILDA). In: Disability and rehabilitation 39, 8, S. 809-816.

Conwell, Yeates/Duberstein, Paul R./Caine, Eric D. (2002): Risk factors for suicide in later life. In: Biological psychiatry 52, 3, S. 193-204.

Conwell, Yeates/Duberstein, Paul R./Cox, Christopher/Herrmann, John H./Forbes, Nicholas T./Caine, Eric D. (1996): Relationships of Age and Axis I Diagnoses in Victims of Completed Suicide: A Psychological Autopsy Study. In: The American Journal of Psychiatry 153, 8, S. 1001-1008.

Cooper, Rachel/Kuh, Diana/Hardy, Rebecca (2010): Objectively measured physical capability levels and mortality: systematic review and meta-analysis. In: BMJ 341, S. c4467.

Copeland, J. R. M./Beekman, A. T. F./Dewey, M. E./Hooijer, C./Jordan, A./Lawlor, B. A./Lobo, A./Magnusson, H./Mann, A. H./Meller, I./Prince, M. J./Reischies, F./Turrina, C./Devries, M. W./Wilson, K. C. M. (1999): Depression in Europe. In: British Journal of Psychiatry 174, 04, S. 312-321.

Cordts, Anette/Spiller, Achim/Nitzko, Sina/Grethe, Harald/Duman, Nuray (2013): Fleischkonsum in Deutschland. Von unbekümmerten Fleischessern, Flexitariern und (Lebensabschnitts-) Vegetariern. In: FleischWirtschaft, 7, S. 59-63.

Credit Suisse Research Institute (2016): Global Wealth Databook 2016.

Crossley, Thomas F./Kennedy, Steven (2002): The reliability of self-assessed health status. In: Journal of health economics 21, 4, S. 643-658.

Cutrona, Carolyn E./Abraham, William T./Russell, Daniel W./Beach, Steven R. H./Gibbons, Frederick X./Gerrard, Meg/Monick, Martha/Philibert, Robert (2015): 
Financial strain, inflammatory factors, and haemoglobin A1c levels in African American women. In: British journal of health psychology 20, 3, S. 662-679.

Ćwirlej-Sozańska, Agnieszka/Sozański, Bernard/Wiśniowska-Szurlej, Agnieszka/Wilmowska-Pietruszyńska, Anna/Ćwirlej-Sozańska, Agnieszka Beata (2018): An assessment of factors related to disability in ADL and IADL in elderly inhabitants of rural areas of south-eastern Poland. In: Annals of Agricultural and Environmental Medicine 25, 3, S. 504-511.

Dahl, Espen/Birkelund, Gunn Elisabeth (1997): Health inequalities in later life in a social democratic welfare state. In: Social science \& medicine 44, 6, S. 871-881.

Dahl, Espen/van der Wel, Kjetil A (2013): Educational inequalities in health in European welfare states: a social expenditure approach. In: Social science \& medicine (1982) 81, S. 60-69.

Daley, Shannon E./Hammen, Constance/Rao, Uma (2000): Predictors of first onset and recurrence of major depression in young women during the 5 years following high school graduation. In: Journal of Abnormal Psychology 109, 3, S. 525-533.

Dawson, Deborah A./Grant, Bridget F./Patricia Chou, S./Pickering, Roger P. (1995): Subgroup variation in U.S. drinking patterns. Results of the 1992 national longitudinal alcohol epidemiologic study. In: Journal of Substance Abuse 7, 3, S. 331344.

Deans, Carrie/Maggert, Keith A. (2015): What do you mean, ,epigenetic“? In: Genetics 199, 4, S. 887-896.

Dearing, Eric/Taylor, Beck A./McCartney, Kathleen (2004): Implications of Family Income Dynamics for Women's Depressive Symptoms During the First 3 Years After Childbirth. In: American Journal of Public Health 94, 8, S. 1372-1377.

Deindl, Christian (2015): Finanzielle Probleme und Stress im Lebenslauf haben einen Einfluss auf das Risiko einer Herzerkrankung im späteren Leben. In: Informationsdienst Soziale Indikatoren 54, S. 12-15.

Deindl, Christian/Brandt, Martina (2015): Social exclusion and support between generations. In: Börsch-Supan, A./Kneip, T./Litwin, H./Myck, M./Weber, G. (Hrsg.): Ageing in Europe - Supporting Policies for an Inclusive Society. Berlin, München, Boston: DE GRUYTER, S. 161-168.

Delgado, Pedro L./Moreno, Francisco A. (2000): Role of norepinephrine in depression. In: Journal of Clinical Psychiatry 61, SUPPL. 1, S. 5-12.

Delhey, J./Dragolov, G. (2014): Why Inequality Makes Europeans Less Happy. The Role of Distrust, Status Anxiety, and Perceived Conflict. In: European Sociological Review 30, 2, S. 151-165.

Delhey, Jan/Newton, Kenneth (2005): Predicting Cross-National Levels of Social Trust. Global Pattern or Nordic Exceptionalism? In: European Sociological Review 21, 4, S. 311-327.

DeSalvo, Karen B./Bloser, Nicole/Reynolds, Kristi/He, Jiang/Muntner, Paul (2006): Mortality prediction with a single general self-rated health question. A meta-analysis. In: Journal of general internal medicine 21, 3, S. 267-275.

Deutsche Bundesbank (2013): Vermögen und Finanzen privaterHaushalte in Deutschland:Ergebnisse der Bundesbankstudie. Monatsbericht 25.

Deutsche Bundesbank (2016): Vermögen und Finanzen privater Haushalte in Deutschland: Ergebnisse der Vermögensbefragung 2014. Monatsbericht 61. 
Dickerson, Sally S./Kemeny, Margaret E. (2004): Acute stressors and cortisol responses: a theoretical integration and synthesis of laboratory research. In: Psychological bulletin 130, 3, S. 355-391.

Diekmann, Andreas/Meyer, Reto (2010): Demokratischer Smog? Eine empirische Untersuchung zum Zusammenhang zwischen Sozialschicht und Umweltbelastungen. In: KZfSS Kölner Zeitschrift für Soziologie und Sozialpsychologie 62, 3, S. 437457.

Diez-Roux, Ana V./Link, Bruce G./Northridge, Mary E. (2000): A multilevel analysis of income inequality and cardiovascular disease risk factors. In: Social science \& medicine 50, 5, S. 673-687.

Dimitrakaki, Christine/Boulamatsis, Dimitris/Mariolis, Anargiros/Kontodimopoulos, Nick/Niakas, Dimitris/Tountas, Yannis (2009): Use of cancer screening services in Greece and associated social factors: results from the nation-wide Hellas Health I survey. In: European journal of cancer prevention the official journal of the European Cancer Prevention Organisation (ECP) 18, 3, S. 248-257.

Ding, Xuejie/Billari, Francesco C./Gietel-Basten, Stuart (2017): Health of midlife and older adults in China: the role of regional economic development, inequality, and institutional setting. In: International journal of public health 62, 8, S. 857-867.

Dolan, Paul/Lordan, Grace (2013): Moving Up and Sliding Down: An Empirical Assessment of the Effect of Social Mobility on Subjective Wellbeing. CEP Discussion Paper.

Domeij, David/Klein, Paul (2002): Public Pensions. To What Extent Do They Account for Swedish Wealth Inequality? In: Review of Economic Dynamics 5, 3, S. 503534.

Dragano, Nico (2016): Arbeit und Gesundheit. In: Richter, M./Hurrelmann, K. (Hrsg.): Soziologie von Gesundheitund Krankheit, S. 167-182.

Dragano, Nico/Siegrist, Johannes (2006): Die Lebenslaufperspektive gesundheitlicher Ungleichheit: Konzepte und Forschungsergebnisse. In: Richter, M./Hurrelmann, K. (Hrsg.): Gesundheitliche Ungleichheit. Wiesbaden: VS Verlag für Sozialwissenschaften, S. 171-184.

Drentea, Patricia/Reynolds, John R. (2015): Where Does Debt Fit in the Stress Process Model? In: Society and Mental Health 5, 1, S. 16-32.

Dunlop, Boadie W./Nemeroff, Charles B. (2007): The role of dopamine in the pathophysiology of depression. In: Archives of general psychiatry 64, 3, S. 327-337.

Dunn, Nick/Inskip, Hazel/Kendrick, Tony/Oestmann, Anne/Barnett, Jane/Godfrey, Keith/Cooper, Cyrus (2008): Does perceived financial strain predict depression among young women? Longitudinal findings from the Southampton Women's Survey. In: Mental health in family medicine 5, 1, S. 15-21.

Elgar, Frank J. (2010): Income inequality, trust, and population health in 33 countries. In: American journal of public health 100, 11, S. 2311-2315.

Elgar, Frank J./Davis, Christopher G./Wohl, Michael J./Trites, Stephen J./Zelenski, John M./Martin, Michael S. (2011): Social capital, health and life satisfaction in 50 countries. In: Health \& place 17, 5, S. 1044-1053.

Elgar, Frank J./Gariépy, Geneviève/Torsheim, Torbjørn/Currie, Candace (2017): Earlylife income inequality and adolescent health and well-being. In: Social science \& medicine (1982) 174, S. 197-208. 
Elstad, Jon Ivar (2010): Indirect health-related selection or social causation? Interpreting the educational differences in adolescent health behaviours. In: Social Theory \& Health 8, 2, S. 134-150.

Engel, George L. (1977): The Need for a New Medical Model: A Challenge for Biomedicine. In: Science 196, 4286, S. 129-136.

Engel, George L. (1980): The clinical application of the biopsychosocial model. In: The American Journal of Psychiatry 137, 5, S. 535-544.

ESS (2004): ESS Round 2: European Social Survey Round 2 Data. Data file edition 3.5. NSD - Norwegian Centre for Research Data, Norway - Data Archive and distributor of ESS data for ESS ERIC.

ESS (2006): ESS Round 3: European Social Survey Round 3 Data. Data file edition 3.6. NSD - Norwegian Centre for Research Data, Norway - Data Archive and distributor of ESS data for ESS ERIC.

ESS (2008): ESS Round 4: European Social Survey Round 4 Data. Data file edition 4.4. NSD - Norwegian Centre for Research Data, Norway - Data Archive and distributor of ESS data for ESS ERIC.

ESS (2010): ESS Round 5: European Social Survey Round 5 Data. Data file edition 3.3. NSD - Norwegian Centre for Research Data, Norway - Data Archive and distributor of ESS data for ESS ERIC.

ESS (2012): ESS Round 6: European Social Survey Round 6 Data. Data file edition 2.3. NSD - Norwegian Centre for Research Data, Norway - Data Archive and distributor of ESS data for ESS ERIC.

ESS (2014): ESS Round 7: European Social Survey Round 7 Data. Data file edition 2.1. NSD - Norwegian Centre for Research Data, Norway - Data Archive and distributor of ESS data for ESS ERIC.

EU (2003): Amtsblatt der Europäischen Union, L 165, 03. Juli 2003. VERORDNUNG (EG) Nr. 1177/2003 DES EUROPÄISCHEN PARLAMENTS UND DES RATESvom 16. Juni 2003 für die Gemeinschaftsstatistik über Einkommen und Lebensbedingungen (EU-SILC).

EU (2015): People in the EU. Who are we and how do we live? 2015 edition. 2015 edition. Statistical books. Luxembourg: Publications Office.

European Social Survey (2016): ESS-1 2002 Documentation Report. Edition 6.5. Bergen: NSD - Norwegian Centre for Research Data, Norway - Data Archive and distributor of ESS data for ESS ERIC.

Eurostat (2016): Gini coefficient of equivalised disposable income - EU-SILC survey.

Eurostat (2018a): At-risk-of-poverty rate by poverty threshold, age and sex - EU-SILC survey.

Eurostat (2018b): Distribution of income by quantiles - EU-SILC survey.

Evenson, Ranae J./Simon, Robin W. (2005): Clarifying the relationship between parenthood and depression. In: Journal of Health and Social Behavior 46, 4, S. 341-358.

Feng, Zhixin/Wang, Wenfei Winnie/Jones, Kelvyn/Li, Yaqing (2012): An exploratory multilevel analysis of income, income inequality and self-rated health of the elderly in China. In: Social science \& medicine (1982) 75, 12, S. 2481-2492. 
Fernández-Niño, Julián Alfredo/Manrique-Espinoza, Betty Soledad/BojorquezChapela, Ietza/Salinas-Rodríguez, Aarón (2014): Income inequality, socioeconomic deprivation and depressive symptoms among older adults in Mexico. In: PloS one 9, 9, S. e108127.

Ferraro, Kenneth F./Kelley-Moore, Jessica A. (2003): Cumulative Disadvantage and Health: Long-Term Consequences of Obesity? In: American Sociological Review 68,5, S. 707-729.

Ferraro, Kenneth F./Su, Ya-ping/Gretebeck, Randall J./Black, David R./Badylak, Stephen F. (2002): Body Mass Index and Disability in Adulthood. A 20-Year Panel Study. In: American Journal of Public Health 92, 5, S. 834-840.

Ferreira, Francisco/Jolliffe, Dean/Prydz, Espen Beer (2015): The international poverty line has just been raised to $\$ 1.90$ a day, but global poverty is basically unchanged. How is that even possible? https://blogs.worldbank.org/developmenttalk/international-poverty-line-has-just-been-raised-190-day-global-poverty-basically-unchanged-how-even [Zugriff: 18.05.2018].

Ferrucci, L./Guralnik, J. M./Simonsick, E./Salive, M. E./Corti, C./Langlois, J. (1996): Progressive versus Catastrophic Disability. A Longitudinal View of the Disablement Process. In: The Journals of Gerontology Series A: Biological Sciences and Medical Sciences 51A, 3, S. M123-M130.

Fiscella, Kevin/Franks, Peter (2000): Individual Income, Income Inequality, Health, and Mortality: What Are the Relationships? In: Health Services Research 35, 1 II, S. 307-318.

Fonseca, Raquel/Kapteyn, Arie/Lee, Jinkook/Zamarro, Gema/Feeney, Kevin (2013): Financial and subjective well-being of older Europeans. In: Börsch-Supan, A./Brandt, M./Litwin, H./Weber, G. (Hrsg.): Active ageing and solidarity between generations in Europe. First results from SHARE after the economic crisis. Berlin: DE GRUYTER, S. 137-146.

Foulds, James/Wells, J. Elisabeth/Mulder, Roger (2014): The association between material living standard and psychological distress: results from a New Zealand population survey. In: The International journal of social psychiatry 60, 8, S. 766-771.

Fox, Ashley M. (2012): The HIV-poverty thesis re-examined: poverty, wealth or inequality as a social determinant of HIV infection in sub-Saharan Africa? In: Journal of biosocial science 44, 4, S. 459-480.

Fraga, Mario F./Ballestar, Esteban/Paz, Maria F./Ropero, Santiago/Setien, Fernando/Ballestar, Maria L./Heine-Suñer, Damia/Cigudosa, Juan C./Urioste, Miguel/Benitez, Javier/Boix-Chornet, Manuel/Sanchez-Aguilera, Abel/Ling, Charlotte/Carlsson, Emma/Poulsen, Pernille/Vaag, Allan/Stephan, Zarko/Spector, Tim D./Wu, Yue-Zhong/Plass, Christoph/Esteller, Manel (2005): Epigenetic differences arise during the lifetime of monozygotic twins. In: Proceedings of the National Academy of Sciences of the United States of America 102, 30, S. 1060410609.

Frank, Christine/Davis, Christopher G./Elgar, Frank J. (2014): Financial strain, social capital, and perceived health during economic recession: a longitudinal survey in rural Canada. In: Anxiety, stress, and coping 27, 4, S. 422-438.

Franzese, Fabio (2015): Slipping into poverty: effects on mental and physical health. In: Börsch-Supan, A./Kneip, T./Litwin, H./Myck, M./Weber, G. (Hrsg.): Ageing 
in Europe - Supporting Policies for an Inclusive Society. Berlin, München, Boston: DE GRUYTER, S. 139-148.

Frederiksen, Henrik/Hjelmborg, Jacob/Mortensen, Jakob/McGue, Matt/Vaupel, James W./Christensen, Kaare (2006): Age trajectories of grip strength: cross-sectional and longitudinal data among 8,342 Danes aged 46 to 102. In: Annals of epidemiology 16,7 , S. 554-562.

French, Declan (2018): Financial strain in the United Kingdom. In: Oxford Economic Papers 70, 1, S. 163-182.

French, Declan/McKillop, Donal (2017): The impact of debt and financial stress on health in Northern Irish households. In: Journal of European Social Policy 27, 5, S. $458-473$.

Frijters, Paul/Ulker, Aydogan (2008): Robustness in health research: do differences in health measures, techniques, and time frame matter? In: Journal of health economics 27,6 , S. 1626-1644.

Frontera, W. R./Hughes, V.A./Fielding, R. A./Fiatarone, M. A./Evans, W. J./Roubenoff, R. (2000): Aging of skeletal muscle: a 12-yr longitudinal study. In: Journal of applied physiology (Bethesda, Md. 1985) 88, 4, S. 1321-1326.

Fuller-Thomson, Esme/Gadalla, Tahany (2008): Income inequality and limitations in activities of daily living: a multilevel analysis of the 2003 American Community Survey. In: Public health 122, 3, S. 221-228.

Gadalla, Tahany M./Fuller-Thomson, Esme (2008): Examining the lag time between state-level income inequality and individual disabilities: a multilevel analysis. In: American journal of public health 98, 12, S. 2187-2190.

Galea, Sandro/Tracy, Melissa/Hoggatt, Katherine J./Dimaggio, Charles/Karpati, Adam (2011): Estimated deaths attributable to social factors in the United States. In: American journal of public health 101, 8, S. 1456-1465.

Gallagher, D./Visser, M./Meersman, R. E. de/Sepúlveda, D./Baumgartner, R. N./Pierson, R. N./Harris, T./Heymsfield, S. B. (1997): Appendicular skeletal muscle mass: effects of age, gender, and ethnicity. In: Journal of applied physiology (Bethesda, Md. 1985) 83, 1, S. 229-239.

Gallo, Joseph J./Rabins, Peter V. (1999): Depression Without Sadness: Alternative Presentations of Depression in Late Life. In: American family physician 60, 3, S. 820-826.

Galobardes, B./Lynch, J. W./Smith, G. Davey (2008): Is the association between childhood socioeconomic circumstances and cause-specific mortality established? Update of a systematic review. In: Journal of epidemiology and community health 62 , 5, S. 387-390.

Gardner, Jonathan/Oswald, Andrew J. (2007): Money and mental wellbeing: a longitudinal study of medium-sized lottery wins. In: Journal of health economics 26,1 , S. 49-60.

Gasche, Martin/Lamla, Bettina (2012): Erwartete Altersarmut in Deutschland: Pessimismus und Fehleinschätzungen - Ergebnisse aus der SAVE-Studie. MEA Discussion Papers. München.

Gathergood, John (2012): Debt and Depression. Causal Links and Social Norm Effects. In: The Economic Journal 122, 563, S. 1094-1114. 
Gemmell, I. (2001): Indoor heating, house conditions, and health. In: Journal of Epidemiology \& Community Health 55, 12, S. 928-929.

George, Linda K./Blazer, Dan G./Hughes, Dana C./Fowler, Nancy (1989): Social Support and the Outcome of Major Depression. In: British Journal of Psychiatry 154, 04, S. 478-485.

Georgiades, Anastasia/Janszky, Imre/Blom, May/László, Krisztina D./Ahnve, Staffan (2009): Financial strain predicts recurrent events among women with coronary artery disease. In: International journal of cardiology 135, 2, S. 175-183.

Geyer, Johannes (2014): Zukünftige Altersarmut. DIW Roundup: Politik im Fokus, 25, Deutsches Institut für Wirtschaftsforschung (DIW), Berlin

Giesselmann, Marco/Windzio, Michael (2014): Paneldaten in der Soziologie. Fixed Effects Paradigma und empirische Praxis in Panelregression und Ereignisanalyse. In: KZfSS Kölner Zeitschrift für Soziologie und Sozialpsychologie 66, 1, S. 95113.

Gilbert, Paul (2006): Evolution and depression: issues and implications. In: Psychological medicine 36, 3, S. 287-297.

Gilbert, Paul/Allan, Steven (1998): The role of defeat and entrapment (arrested flight) in depression: an exploration of an evolutionary view 28, 3, S. 585-598.

Gill, Thomas M./Gahbauer, Evelyne A./Han, Ling/Allore, Heather G. (2010): Trajectories of disability in the last year of life. In: The New England journal of medicine 362, 13, S. 1173-1180.

Giordano, Giuseppe N./Lindstrom, Martin (2010): The impact of changes in different aspects of social capital and material conditions on self-rated health over time: a longitudinal cohort study. In: Social science \& medicine (1982) 70, 5, S. 700-710.

Glaser, Ronald/Kiecolt-Glaser, Janice K. (2005): Stress-induced immune dysfunction: implications for health. In: Nature reviews. Immunology 5, 3, S. 243-251.

Glymour, M. Maria (2008): Sensitive periods and first difference models: integrating etiologic thinking into econometric techniques: a commentary on Clarkwest's „Neo-materialist theory and the temporal relationship between income inequality and longevity change“". In: Social science \& medicine (1982) 66, 9, S. 1895-902; discussion 1903-8.

Glymour, M. Maria/Avendano, Mauricio/Kawachi, Ichiro (2014): Socioeconomic status and health. In: Berkman, L. F./Kawachi, I./Glymour, M. M. (Hrsg.): Social epidemiology. 2 ed. New York: Oxford University Press, S. 17-62.

Gobbens, Robbert J. J./van Assen, Marcel A.L.M. (2014): The Prediction of ADL and IADL Disability Using Six Physical Indicators of Frailty: A Longitudinal Study in the Netherlands. In: Current gerontology and geriatrics research 2014, S. 358137.

Goerke, Laszlo/Pannenberg, Markus (2015): Direct evidence for income comparisons and subjective well-being across reference groups. In: Economics Letters 137, S. 95-101.

Goldberg, Lewis R. (1993): The structure of phenotypic personality traits. In: American Psychologist 48, 1, S. 26-34.

Graham, Hilary (2002): Building an inter-disciplinary science of health inequalities. The example of lifecourse research. In: Social science \& medicine 55, 11, S. 20052016. 
Gravelle, Hugh (1998): How much of the relation between population mortality and unequal distribution of income is a statistical artefact? In: BMJ: British Medical Journal 316, 7128, S. 382-385.

Gravelle, Hugh/Sutton, Matt (2009): Income, relative income, and self-reported health in Britain 1979-2000. In: Health economics 18, 2, S. 125-145.

Greenberg, Neil/Chen, Thomas/Crews, David (1984): Social status, gonadal state, and the adrenal stress response in the lizard, Anolis carolinensis. In: Hormones and Behavior 18, 1, S. 1-11.

Greene, William (2002): The Bias of the Fixed Effects Estimator in Nonlinear Models. http://citeseerx.ist.psu.edu/viewdoc/download;jsessionid=CD087B8D48EB2EF085877349A7A03DA9?doi=10.1.1.463.9383\&rep= rep1\&type=pdf [Zugriff: 29.07.2018].

Groenemeyer, Axel/Ratzka, Melanie (2012): Armut, Deprivation und Exklusion als soziales Problem. In: Albrecht, G./Groenemeyer, A. (Hrsg.): Handbuch soziale Probleme. Wiesbaden: VS Verlag für Sozialwissenschaften, S. 367-432.

Groh-Samberg, Olaf (2010): Armut verfestigt sich-ein missachteter Trend. In: Aus Politik und Zeitgeschichte 51, 52, S. 9-15.

Grönqvist, Hans/Johansson, Per/Niknami, Susan (2012): Income inequality and health: lessons from a refugee residential assignment program. In: Journal of health economics 31, 4, S. 617-629.

Grossman, Michael (1972): On the Concept of Health Capital and the Demand for Health. In: Journal of Political Economy 80, 2, S. 223-255.

Grundy, E./Holt, G. (2001): The socioeconomic status of older adults. How should we measure it in studies of health inequalities? In: Journal of Epidemiology \& Community Health 55, 12, S. 895-904.

Grundy, Emily M. D./Tomassini, Cecilia (2010): Marital history, health and mortality among older men and women in England and Wales. In: BMC public health 10, S. 554.

Grünheid, Evelyn (2004): Einflüsse der Einkommenslage auf Gesundheit und Gesundheitsverhalten: Ergebnisse desLebenserwartungssurveys des BiB. Materialien zur Bevölkerungswissenschaft. Wiesbaden.

Gunasekara, Fiona Imlach/Carter, Kristie/Blakely, Tony (2012): Comparing self-rated health and self-assessed change in health in a longitudinal survey: which is more valid? In: Social science \& medicine (1982) 74, 7, S. 1117-1124.

Guralnik, Jack M./Ferrucci, Luigi/Balfour, Jennifer L./Volpato, Stefano/Di Iorio, Angelo (2001): Progressive versus Catastrophic Loss of the Ability to Walk. Implications for the Prevention of Mobility Loss. In: Journal of the American Geriatrics Society 49, 11, S. 1463-1470.

Gutiérrez-Colosía, M. R./Salvador-Carulla, L./Salinas-Pérez, J. A./García-Alonso, C. R./Cid, J./Salazzari, D./Montagni, I./Tedeschi, F./Cetrano, G./Chevreul, K./Kalseth, J./Hagmair, G./Straßmayr, C./Park, A. L./Sfectu, R./Ala-Nikkola, T./González-Caballero, J. L./Rabbi, L./Kalseth, B./Amaddeo, F. (2017): Standard comparison of local mental health care systems in eight European countries. In: Epidemiology and psychiatric sciences, S. 1-14.

Haan, Peter/Stichnoth, Holger/Blömer, Maximilian/Buslei, Hermann/Geyer, Johannes/Krolage, Carla/Müller, Kai-Uwe (2017): Entwicklung der Altersarmut bis 
2036: Trends,Risikogruppen und Politikszenarien. ZEW-Gutachten und Forschungsberichte. Gütersloh.

Hagemann, Helmut (1998): Umweltverhalten zwischen Arbeit, Einkommen und Lebensstil. Konsumentenverhalten im Spannungsfeld von subjektiven Orientierungsmustern und Arbeitszeit- und Einkommensveränderungen. Schriftenreihe des IÖW / Institut für Ökologische Wirtschaftsforschung gGmbH, Band 131. Berlin: IÖW.

Haig, D. (2004): The (dual) origin of epigenetics. In: Cold Spring Harbor symposia on quantitative biology 69, S. 67-70.

Hanemann, Felizia (2018): Labor market inactivity and health in old age: What role do institutional, occupational and social factors play? Dissertation. München: Technische Universität München.

Hank, Karsten/Wagner, Michael (2013): Parenthood, Marital Status, and Well-Being in Later Life. Evidence from SHARE. In: Social Indicators Research 114, 2, S. 639653.

Hansen, Thomas/Slagsvold, Britt/Moum, Torbjørn (2009): Childlessness and Psychological Well-Being in Midlife and Old Age. An Examination of Parental Status Effects Across a Range of Outcomes. In: Social Indicators Research 94, 2, S. $343-$ 362.

Hardie, Jessica Halliday/Lucas, Amy (2010): Economic Factors and Relationship Quality Among Young Couples: Comparing Cohabitation and Marriage. In: Journal of marriage and the family $72,5, \mathrm{~S} .1141-1154$.

Hardy, Susan E./Gill, Thomas M. (2004): Recovery from disability among communitydwelling older persons. In: JAMA 291, 13, S. 1596-1602.

Harkness, Janet (2005): SHARE translation procedures and translation assessment. In: Börsch-Supan, A. (Hrsg.): The survey of health, ageing, and retirement in Europe. Methodology ;. Mannheim: MEA, S. 24-27.

Härter, Martin/Baumeister, Harald (2007): Ätiologie psychischer Störungen bei chronischen körperlichen Erkrankungen. In: Härter, M./Baumeister, H./Bengel, J. (Hrsg.): Psychische Störungen bei körperlichen Erkrankungen. Berlin, Heidelberg: Springer Berlin Heidelberg, S. 1-13.

Hauser, Richard (2012): Das Maß der Armut: Armutsgrenzen im sozialstaatlichen Kontext - Der sozialstatistische Diskurs. In: Huster, E.-U./Boeckh, J./Mogge-Grotjahn, H. (Hrsg.): Handbuch Armut und Soziale Ausgrenzung. Wiesbaden: VS Verlag für Sozialwissenschaften, S. 122-146.

Hauser, Richard/Neumann, Udo (1992): Armut in der Bundesrepublik Deutschland. In: Leibfried, S./Voges, W. (Hrsg.): Armut im modernen Wohlfahrtsstaat. Zeitschrift für Soziologie und Sozialpsychologie (Sonderhefte), Band 32. Wiesbaden: VS Verlag für Sozialwissenschaften, S. 237-271.

Hays, J. C./Saunders, W. B./Flint, E. P./Kaplan, B. H./Blazer, Dan G. 2nd (1997): Social support and depression as risk factors for loss of physical function in late life. In: Aging \& Mental Health 1, 3, S. 209-220.

Head, J. (2004): The psychosocial work environment and alcohol dependence. A prospective study. In: Occupational and Environmental Medicine 61, 3, S. 219-224.

Heflin, Colleen M./Iceland, John (2009): Poverty, Material Hardship and Depression. In: Social science quarterly 90, 5, S. 1051-1071. 
Heim, Christine/Binder, Elisabeth B. (2012): Current research trends in early life stress and depression: review of human studies on sensitive periods, gene-environment interactions, and epigenetics. In: Experimental neurology 233, 1, S. 102-111.

Heinonen, Kati/Räikkönen, Katri/Matthews, Karen A./Scheier, Michael F./Raitakari, Olli T./Pulkki, Laura/Keltikangas-Järvinen, Liisa (2006): Socioeconomic status in childhood and adulthood: associations with dispositional optimism and pessimism over a 21-year follow-up. In: Journal of personality 74, 4, S. 1111-1126.

Hemingway, Harry/Marmot, Michael (1999): Psychosocial factors in the aetiology and prognosis of coronary heart disease: systematic review of prospective cohort studies. In: BMJ, 318, S. 1460-1467.

Hiilamo, Aapo/Grundy, Emily (2018): Household debt and depressive symptoms among older adults in three continental European countries. In: Ageing and Society 13, S. 1-27.

Hildebrand, Vincent/van Kerm, Philippe (2009): Income Inequality and Self-Rated Health Status. Evidence From the European Community Household Panel. In: Demography 46, 4, S. 805-825.

Hill, Terrence D./Jorgenson, Andrew (2018): Bring out your dead!: A study of income inequality and life expectancy in the United States, 2000-2010. In: Health \& place 49, S. 1-6.

Hoffmann, Elke/Menning, Sonja/Schelhase, Torsten (2009): 1.2 Demografische Perspektiven zum Altern und zum Alter. In: Robert Koch-Institut (Hrsg.): Gesundheitsberichterstattung des Bundes. Gesundheit und Krankheit im Alter. Berlin, S. 31-61.

Hoffmann, Rasmus/Kröger, Hannes/Pakpahan, Eduwin (2018a): Pathways between socioeconomic status and health. Does health selection or social causation dominate in Europe? In: Advances in Life Course Research 36, S. 23-36.

Hoffmann, Rasmus/Kröger, Hannes/Pakpahan, Eduwin (2018b): The reciprocal relationship between material factors and health in the life course. Evidence from SHARE and ELSA. In: European Journal of Ageing 75, S. 1303.

Holt-Lunstad, Julianne/Smith, Timothy B./Layton, J. Bradley (2010): Social relationships and mortality risk: a meta-analytic review. In: PLoS medicine 7, 7, S. e1000316.

Hong, Jihyung/Knapp, Martin/McGuire, Alistair (2011): Income-related inequalities in the prevalence of depression and suicidal behaviour. A 10-year trend following economic crisis. In: World Psychiatry 10, 1, S. 40-44.

Horrace, William C./Oaxaca, Ronald L. (2006): Results on the bias and inconsistency of ordinary least squares for the linear probability model. In: Economics Letters 90,3, S. 321-327.

House, James S. (1981): Work stress and social support. Reading, MA: Addison-Wesley.

Hu, Yannan/van Lenthe, Frank J./Mackenbach, Johan P. (2015): Income inequality, life expectancy and cause-specific mortality in 43 European countries, 1987-2008: a fixed effects study. In: European journal of epidemiology 30, 8, S. 615-625.

Huijts, T./Kraaykamp, G./Subramanian, S. V. (2013): Childlessness and Psychological Well-Being in Context. A Multilevel Study on 24 European Countries. In: European Sociological Review 29, 1, S. 32-47. 
Huijts, Tim/Eikemo, Terje Andreas/Skalická, Vera (2010): Income-related health inequalities in the Nordic countries: examining the role of education, occupational class, and age. In: Social science \& medicine (1982) 71, 11, S. 1964-1972.

Huisman, Martijn/Kunst, Anton E./Mackenbach, Johan P. (2003): Socioeconomic inequalities in morbidity among the elderly; a European overview. In: Social science $\&$ medicine 57, 5, S. 861-873.

Ichida, Yukinobu/Kondo, Katsunori/Hirai, Hiroshi/Hanibuchi, Tomoya/Yoshikawa, Goshu/Murata, Chiyoe (2009): Social capital, income inequality and self-rated health in Chita peninsula, Japan: a multilevel analysis of older people in $25 \mathrm{com}-$ munities. In: Social science \& medicine (1982) 69, 4, S. 489-499.

Idler, Ellen L./Benyamini, Yael (1997): Self-Rated Health and Mortality. A Review of Twenty-Seven Community Studies. In: Journal of Health and Social Behavior 38, 1, S. 21.

IHME (2017): Global Burden of Disease Study 2016 (GBD 2016) Results. Seattle. http://ghdx.healthdata.org/gbd-results-tool.

Imlach Gunasekara, Fiona/Carter, Kristie/Blakely, Tony (2011): Change in income and change in self-rated health: Systematic review of studies using repeated measures to control for confounding bias. In: Social science \& medicine 72, 2, S. 193-201.

Imlach Gunasekara, Fiona/Carter, Kristie N./Crampton, Peter/Blakely, Tony (2013): Income and individual deprivation as predictors of health over time. In: International journal of public health 58, 4, S. 501-511.

Insler, Michael (2014): The Health Consequences of Retirement. In: Journal of Human Resources 49, 1, S. 195-233.

Irz, Xavier/Fratiglioni, Laura/Kuosmanen, Nataliya/Mazzocchi, Mario/Modugno, Lucia/Nocella, Giuseppe/Shakersain, Behnaz/Traill, W. Bruce/Xu, Weili/Zanello, Giacomo (2014): Sociodemographic determinants of diet quality of the EU elderly: a comparative analysis in four countries. In: Public health nutrition 17, 5, S. 11771189.

James, Oliver (2007): Affluenza. London: Vermilion.

Janssen, Ian/Heymsfield, Steven B./Wang, Zimian M./Ross, Robert (2000): Skeletal muscle mass and distribution in 468 men and women aged 18-88 yr. In: Journal of applied physiology (Bethesda, Md. 1985) 89, 1, S. 81-88.

Johnson, Elizabeth O./Kamilaris, Themis C./Chrousos, George P./Gold, Philip W. (1992): Mechanisms of stress. A dynamic overview of hormonal and behavioral homeostasis. In: Neuroscience \& Biobehavioral Reviews 16, 2, S. 115-130.

Johnson, Sheri L./Wibbels, Erik/Wilkinson, Richard (2015): Economic inequality is related to cross-national prevalence of psychotic symptoms. In: Social psychiatry and psychiatric epidemiology 50, 12, S. 1799-1807.

Jones, Andrew M./Wildman, John (2008): Health, income and relative deprivation: evidence from the BHPS. In: Journal of health economics 27, 2, S. 308-324.

Jones, Nicholas R. V./Conklin, Annalijn I./Suhrcke, Marc/Monsivais, Pablo (2014): The growing price gap between more and less healthy foods: analysis of a novel longitudinal UK dataset. In: PloS one 9, 10, S. e109343.

Jongenelis, K./Pot, A. M./Eisses, A. M. H./Beekman, A. T. F./Kluiter, H./Ribbe, M. W. (2004): Prevalence and risk indicators of depression in elderly nursing home patients: the AGED study. In: Journal of Affective Disorders 83, 2-3, S. 135-142. 
Jürges, Hendrik (2007): True health vs response styles: exploring cross-country differences in self-reported health. In: Health economics 16, 2, S. 163-178.

Jürges, Hendrik (2009): Bildungsbezogene Ungleichheit in der Gesundheit Älterer: Eine international vergleichende Analyse. In: Börsch-Supan, A./Hank, K./Jürges, H./Schrader, M. (Hrsg.): 50plus in Deutschland und Europa. Ergebnisse des Survey of Health, Ageing and Retirement in Europe. Wiesbaden: VS Verlag für Sozialwissenschaften / GWV Fachverlage GmbH, Wiesbaden, S. 149-173.

Jürges, Hendrik (2015): Health insurance coverage and access to care among European elders: crossnational differences and social gradients. In: Börsch-Supan, A./Kneip, T./Litwin, H./Myck, M./Weber, G. (Hrsg.): Ageing in Europe - Supporting Policies for an Inclusive Society. Berlin, München, Boston: DE GRUYTER, S. 301312.

Jürges, Hendrik/Avendano, Mauricio/Mackenbach, Johan P. (2008): Are different measures of self-rated health comparable? An assessment in five European countries. In: European journal of epidemiology 23, 12, S. 773-781.

Jylhä, Marja (2009): What is self-rated health and why does it predict mortality? Towards a unified conceptual model. In: Social science \& medicine (1982) 69, 3, S. 307-316.

Jylhä, Marja/Guralnik, Jack M./Balfour, Jennifer L./Fried, Linda P. (2001): Walking Difficulty, Walking Speed, and Age as Predictors of Self-Rated Health. The Women's Health and Aging Study. In: The Journals of Gerontology Series A: Biological Sciences and Medical Sciences 56, 10, S. M609-M617.

Kahn, Robert S./Wise, Paul H./Kennedy, Bruce P./Kawachi, Ichiro (2000): State income inequality, household income, and maternal mental and physical health. Cross sectional national survey. In: BMJ 321, 7272, S. 1311-1315.

Kahneman, Daniel/Tversky, Amos (1979): Prospect Theory: An Analysis of Decision under Risk. In: Econometrica 47, 2, S. 263-292.

Kalmijn, Matthijs/Saraceno, Chiara (2008): A comparative perspective on intergenerational support. In: European Societies 10, 3, S. 479-508.

Karanikolos, Marina/Mladovsky, Philipa/Cylus, Jonathan/Thomson, Sarah/Basu, Sanjay/Stuckler, David/Mackenbach, Johan P./McKee, Martin (2013): Financial crisis, austerity, and health in Europe. In: The Lancet 381, 9874, S. 1323-1331.

Karlsson, Martin/Nilsson, Therese/Lyttkens, Carl Hampus/Leeson, George (2010): Income inequality and health: importance of a cross-country perspective. In: Social science \& medicine (1982) 70, 6, S. 875-885.

Kawachi, Ichiro/Adler, Nancy E./Dow, William H. (2010): Money, schooling, and health: Mechanisms and causal evidence. In: Annals of the New York Academy of Sciences 1186, S. 56-68.

Kawachi, Ichiro/Kennedy, Bruce P./Lochner, Kimberly/Prothrow-Stith, Deborah (1997): Social capital, income inequality, and mortality. In: American Journal of Public Health 87, 9, S. 1491-1498.

Keller, M. L./Leventhal, E. A./Larson, B. (1989): Aging: the lived experience. In: International journal of aging \& human development 29, 1, S. 67-82.

Kelly, Yvonne/Kelly, John/Sacker, Amanda (2013): Time for bed: associations with cognitive performance in 7-year-old children: a longitudinal population-based study. In: Journal of epidemiology and community health 67, 11, S. 926-931. 
Kendler, Kenneth S./Hettema, John M./Butera, Frank/Gardner, Charles O./Prescott, Carol A. (2003): Life event dimensions of loss, humiliation, entrapment, and danger in the prediction of onsets of major depression and generalized anxiety. In: Archives of general psychiatry 60, 8, S. 789-796.

Kennedy, B. P./Kawachi, I./Glass, R./Prothrow-Stith, D. (1998): Income distribution, socioeconomic status, and self rated health in the United States. Multilevel analysis. In: BMJ 317, 7163, S. 917-921.

Kercher, Amy J./Rapee, Ronald M./Schniering, Carolyn A. (2009): Neuroticism, life events and negative thoughts in the development of depression in adolescent girls. In: Journal of abnormal child psychology 37, 7, S. 903-915.

Kickbusch, Ilona (1999): Der Gesundheitsbegriff der Weltgesundheitsorganisation. In: Häfner, H. (Hrsg.): Gesundheit — unser höchstes Gut? Berlin, Heidelberg: Springer Berlin Heidelberg, S. 275-286.

Kiecolt-Glaser, Janice K./Loving, Timothy J./Stowell, Jeffrey R./Malarkey, William B./Lemeshow, Stanley/Dickinson, Stephanie L./Glaser, Ronald (2005): Hostile marital interactions, proinflammatory cytokine production, and wound healing. In: Archives of general psychiatry 62, 12, S. 1377-1384.

Kim, Jaeseung/Shim, Joyce/Lee, RaeHyuck (2016): Material hardship and depression among low-income households in South Korea. Differences by household type. In: International Journal of Social Welfare 25, 2, S. 187-198.

Kim, Youngmi/Park, Aely/Kim, Kyeongmo (2018): Food insecurity and depressive symptoms of older adults living alone in South Korea. In: Ageing and Society 48, S. 1-17.

Kinzl, J. F. (2013): Psychische Erkrankungen bei Frauen und Männern im Alter. In: Zeitschrift fur Gerontologie und Geriatrie 46, 6, S. 526-531.

Kirkbride, James B./Jones, Peter B./Ullrich, Simone/Coid, Jeremy W. (2014): Social deprivation, inequality, and the neighborhood-level incidence of psychotic syndromes in East London. In: Schizophrenia bulletin 40, 1, S. 169-180.

Kistler, Ernst/Trischler, Falko (2012): Altersarmut und Methusalem-Lüge. Wie die Senkung des Rentenniveaus mit demographischen Mythen begründet wird. In: Butterwegge, C./Bosbach, G./Birkwald, M. W. (Hrsg.): Armut im Alter. Probleme und Perspektiven der sozialen Sicherung. Frankfurt am Main, New York: Campus, S. 163-174.

Kivimäki, Mika/Leino-Arjas, Päivi/Luukkonen, Ritva/Riihimäki, Hilkka/Vahtera, Jussi/Kirjonen, Juhani (2002): Work stress and risk of cardiovascular mortality. Prospective cohort study of industrial employees. In: BMJ 325, 7369, S. 857.

Klein, Daniel N./Kotov, Roman/Bufferd, Sara J. (2011): Personality and depression: explanatory models and review of the evidence. In: Annual review of clinical psychology 7, S. 269-295.

Klein, Thomas (2009): Determinanten der Sportaktivität und der Sportart im Lebenslauf. In: Kölner Zeitschrift für Soziologie und Sozialpsychologie 61, 1, S. 1-32.

Klein, Thomas (2016): Sozialstrukturanalyse. Eine Einführung. 2., überarbeitete Auflage. Weinheim, Basel: Beltz Verlagsgruppe.

Klein, Thomas/Unger, Rainer (2001): Einkommen, Gesundheit und Mortalität in Deutschland, Grossbritannien und den USA. In: KZfSS Kölner Zeitschrift für Soziologie und Sozialpsychologie 53, 1, S. 96-110. 
Knabe, André/Fischer, Hagen/Klärner, Andreas (2018): Armut als relationales Konstrukt: Die (Re-)Produktion sozialer Ungleichheiten durch Stigmatisierung und Kontrollversuche in sozialen Netzwerken. In: Behrmann, L./Eckert, F./Gefken, A. (Hrsg.): 'Doing Inequality'. Prozesse sozialer Ungleichheit im Blick qualitativer Sozialforschung. 1. Auflage 2018. Sozialstrukturanalyse. Wiesbaden: Springer Fachmedien Wiesbaden, S. 167-190.

Knesebeck, Olaf von dem/Lüschen, Günther/Cockerham, William C./Siegrist, Johannes (2003): Socioeconomic status and health among the aged in the United States and Germany. A comparative cross-sectional study. In: Social science \& medicine 57,9, S. $1643-1652$.

Kolb, Kathrin/Skopek, Nora/Blossfeld, Hans-Peter (2013): The Two Dimensions of Housing Inequality in Europe Are High Home Ownership Rates an Indicator of Low Housing Values? 339 kB / Comparative Population Studies, Vol 38, No 4 (2013): Ageing Populations, Reversing Early Retirement and the Economic Situation of Older People in Modern Societies.

Kondo, Naoki/Sembajwe, Grace/Kawachi, Ichiro/van Dam, Rob M./Subramanian, S. V./Yamagata, Zentaro (2009): Income inequality, mortality, and self rated health: meta-analysis of multilevel studies. In: BMJ (Clinical research ed.) 339, S. b4471.

Kondo, Naoki/van Dam, Rob M./Sembajwe, Grace/Subramanian, S. V./Kawachi, Ichiro/Yamagata, Zentaro (2012): Income inequality and health: the role of population size, inequality threshold, period effects and lag effects. In: Journal of epidemiology and community health 66,6 , S. e11.

Kraemer, Klaus (2007): Umwelt und soziale Ungleichheit. In: Leviathan 35, 3, S. 348 372.

Kragten, Nigel/Rözer, Jesper (2017): The Income Inequality Hypothesis Revisited. Assessing the Hypothesis Using Four Methodological Approaches. In: Social Indicators Research 131, 3, S. 1015-1033.

Kristensen, Pål/Weisæth, Lars/Heir, Trond (2012): Bereavement and mental health after sudden and violent losses: a review. In: Psychiatry 75, 1, S. 76-97.

Kröger, Hannes/Pakpahan, Eduwin/Hoffmann, Rasmus (2015): What causes health inequality? A systematic review on the relative importance of social causation and health selection. In: European journal of public health 25, 6, S. 951-960.

Kroll, Lars Eric/Ziese, Thomas (2009): 2.5 Kompression oder Expansion der Morbidität? In: Robert Koch-Institut (Hrsg.): Gesundheitsberichterstattung des Bundes. Gesundheit und Krankheit im Alter. Berlin, S. 105-112.

Kuh, D. (2003): Life course epidemiology. In: Journal of Epidemiology \& Community Health 57, 10, S. 778-783.

Kumari, Meena/Head, Jenny/Marmot, Michael (2004): Prospective study of social and other risk factors for incidence of type 2 diabetes in the Whitehall II study. In: Archives of internal medicine 164, 17, S. 1873-1880.

Kümpers, Susanne/Alisch, Monika (2018): Altern und Soziale Ungleichheiten: Teilhabechancen und Ausgrenzungsrisiken. In: Huster, E.-U./Boeckh, J./Mogge-Grotjahn, H. (Hrsg.): Handbuch Armut und soziale Ausgrenzung. Wiesbaden: Springer Fachmedien Wiesbaden, S. 597-618.

Kuntz, B./Zeiher, J./Hoebel, J./Lampert, T. (2016): Soziale Ungleichheit, Rauchen und Gesundheit. In: Suchttherapie 17, 03, S. 115-123. 
Kuo, Chun-Tung/Chiang, Tung-liang (2013): The association between relative deprivation and self-rated health, depressive symptoms, and smoking behavior in Taiwan. In: Social science \& medicine (1982) 89, S. 39-44.

Kwetkat, A. (2010): Immunologie im Alter. In: Prävention und Gesundheitsförderung 5, S1, S. 46-50.

La Ferrara, Eliana (2002): Inequality and group participation. Theory and evidence from rural Tanzania. In: Journal of Public Economics 85, 2, S. 235-273.

Ladin, Keren (2008): Risk of late-life depression across 10 European Union countries: deconstructing the education effect. In: Journal of aging and health 20, 6, S. 653670 .

Ladin, Keren/Daniels, Norman/Kawachi, Ichiro (2010): Exploring the relationship between absolute and relative position and late-life depression: evidence from $10 \mathrm{Eu}-$ ropean countries. In: The Gerontologist 50, 1, S. 48-59.

Laferrère, Anne/van den Bosch, Karel (2015): Unmet need for long-term care and social exclusion. In: Börsch-Supan, A./Kneip, T./Litwin, H./Myck, M./Weber, G. (Hrsg.): Ageing in Europe - Supporting Policies for an Inclusive Society. Berlin, München, Boston: DE GRUYTER, S. 331-341.

Lallukka, T./Laaksonen, M./Rahkonen, O./Roos, E./Lahelma, E. (2007): Multiple socio-economic circumstances and healthy food habits. In: European journal of clinical nutrition 61, 6, S. 701-710.

Lamla, Bettina/Gasche, Martin (2013): Erwarteter Bezug von Grundsicherung im Alter. Verhaltensunterschiede und Fehleinschätzungen. In: Schmollers Jahrbuch 133, 4, S. $539-562$.

Lampert, T./Kroll, L. E. (2006): Einkommensdifferenzen in der Gesundheit und Lebenserwartung - Quer- und Längsschnittbefunde des Sozio-oekonomischen Panels (SOEP). In: Gesundheitswesen (Bundesverband der Arzte des Offentlichen Gesundheitsdienstes (Germany)) 68, 4, S. 219-230.

Lampert, T./Kroll, L. E./Müters, S./Schumann, M. (2017): Soziale Ungleichheit, Arbeit und Gesundheit. In: Badura, B./Ducki, A./Schröder, H./Klose, J./Meyer, M. (Hrsg.): Fehlzeiten-Report 2017. Berlin, Heidelberg: Springer Berlin Heidelberg, S. 23-35.

Lampert, Thomas (2011): Rauchen - Aktuelle Entwicklungen bei Erwachsenen. GBE kompakt 2(4).

Lampert, Thomas (2016): Soziale Ungleichheit und Gesundheit. In: Richter, M./Hurrelmann, K. (Hrsg.): Soziologie von Gesundheit und Krankheit. Wiesbaden: Springer Fachmedien Wiesbaden, S. 121-137.

Lancee, Bram/Van de Werfhorst, Herman G. (2012): Income inequality and participation: A comparison of 24 European countries. In: Social science research 41, 5, S. 1166-1178.

Lange, Clare/Byrd, Mark (1998): The Relationship Between Perceptions of Financial Distress and Feelings of Psychological Well-being in New Zealand University Students. In: International Journal of Adolescence and Youth 7, 3, S. 193-209.

Latif, Ehsan (2015): Income Inequality and Health. Panel Data Evidence from Canada. In: The B.E. Journal of Economic Analysis \& Policy 15, 2, S. 249.

Lau, Jennifer Y. F./Eley, Thalia C. (2010): The genetics of mood disorders. In: Annual review of clinical psychology 6, S. 313-337. 
Laux, G. (2003): Affektive Störungen: Einleitung und Übersicht. In: Psychiatrie und Psychotherapie, S. 1153-1158.

Layte, R. (2012): The Association Between Income Inequality and Mental Health. Testing Status Anxiety, Social Capital, and Neo-Materialist Explanations. In: European Sociological Review 28, 4, S. 498-511.

Layte, R./Whelan, C. T. (2014): Who Feels Inferior? A Test of the Status Anxiety Hypothesis of Social Inequalities in Health. In: European Sociological Review 30, 4, S. 525-535.

Lee, Amanda J./Kane, Sarah/Ramsey, Rebecca/Good, Elizabeth/Dick, Mathew (2016): Testing the price and affordability of healthy and current (unhealthy) diets and the potential impacts of policy change in Australia. In: BMC public health 16, S. 315.

Leger, Kate A./Charles, Susan T./Almeida, David M. (2018): Let It Go: Lingering Negative Affect in Response to Daily Stressors Is Associated With Physical Health Years Later. In: Psychological science, S. 956797618763097.

Leigh, Andrew/Jencks, Christopher (2007): Inequality and mortality: long-run evidence from a panel of countries. In: Journal of health economics 26, 1, S. 1-24.

Leisering, Lutz/Buhr, Petra (2012): Dynamik von Armut. In: Huster, E.-U./Boeckh, J./Mogge-Grotjahn, H. (Hrsg.): Handbuch Armut und Soziale Ausgrenzung. Wiesbaden: VS Verlag für Sozialwissenschaften, S. 147-163.

Lennon, Mary Clare/Limonic, Laura (2010): Work and Unemployment as Stressors. In: Scheid, T. L./Brown, T. N. (Hrsg.): A handbook for the study of mental health. Social contexts, theories, and systems. 2nd ed. Cambridge, New York: Cambridge University Press, S. 213-225.

Lenz, Karl (2009): Soziologie der Zweierbeziehung. Eine Einführung. 4. Aufl. Lehrbuch. Wiesbaden: VS Verlag für Sozialwissenschaften.

Leopold, Liliya/Engelhardt, Henriette/Engelhartdt, Henriette (2013): Education and physical health trajectories in old age. Evidence from the Survey of Health, Ageing and Retirement in Europe (SHARE). In: International journal of public health 58, 1, S. 23-31.

Lerner, Debra/Henke, Rachel Mosher (2008): What does research tell us about depression, job performance, and work productivity? In: Journal of occupational and environmental medicine 50, 4, S. 401-410.

Lesch, Klaus Peter (2004): Gene-environment interaction and the genetics of depression. In: Journal of Psychiatry and Neuroscience 29, 3, S. 174-184.

Leßmann, Ortrud (2006): Lebenslagen und Verwirklichungschancen (capability) - Verschiedene Wurzeln, ähnliche Konzepte. In: Vierteljahrshefte zur Wirtschaftsforschung 75, 1, S. 30-42.

Lewinsohn, Peter M./Allen, Nicholas B./Seeley, John R./Gotlib, Ian H. (1999): First onset versus recurrence of depression. Differential processes of psychosocial risk. In: Journal of Abnormal Psychology 108, 3, S. 483-489.

Li, Hongbin/Zhu, Yi (2006): Income, income inequality, and health. Evidence from China. In: Journal of Comparative Economics 34, 4, S. 668-693.

Lien, Donald/Rearden, David (1990): PRACTITIONERS' CORNER. A Remark on 'An Advantage of the Linear Probability Model over Probit or Logit'. In: Oxford Bulletin of Economics and Statistics 52, 2, S. 223-225. 
Lillard, Dean R./Burkhauser, Richard V./Hahn, Markus H./Wilkins, Roger (2015): Does early-life income inequality predict self-reported health in later life? Evidence from the United States. In: Social science \& medicine (1982) 128, S. $347-$ 355.

Lindau, Stacy Tessler/Laumann, Edward O./Levinson, Wendy/Waite, Linda J. (2003): Synthesis of Scientific Disciplines in Pursuit of Health. The Interactive Biopsychosocial Model. In: Perspectives in Biology and Medicine 46, 3x, S. S74-S86.

Lindeboom, Maarten/Portrait, France/van den Berg, Gerard J (2002): An econometric analysis of the mental-health effects of major events in the life of older individuals. In: Health economics 11, 6, S. 505-520.

Lindqvist, Erik/Östling, Robert/Cesarini, David (2018): Long-run Effects of Lottery Wealth on Psychological Well-being. Cambridge, MA: National Bureau of Economic Research.

Lischka, Anna-Marie (2009): Soziale Partizipation und Kontextfaktoren bei Patienten mit psychischen Störungen. Dissertation: Freie Universität Berlin/Medizinische Fakultät Charité - Universitätsmedizin Berlin.

Litwin, Howard/Sapir, Eliyahu V. (2009): Perceived income adequacy among older adults in 12 countries: findings from the survey of health, ageing, and retirement in Europe. In: The Gerontologist 49, 3, S. 397-406.

Litwin, Howard/Stoeckel, Kimberly J. (2013): Social network and mobility improvement among older Europeans: the ambiguous role of family ties. In: European journal of ageing 10, 3, S. 159-169.

Lofors, Jonas/Ramírez-León, Vania/Sundquist, Kristina (2006): Neighbourhood income and anxiety: a study based on random samples of the Swedish population. In: European journal of public health 16, 6, S. 633-639.

Lorant, Vincent/Croux, Christophe/Weich, Scott/Deliège, Denise/Mackenbach, Johan/Ansseau, Marc (2007): Depression and socio-economic risk factors: 7-year longitudinal population study. In: The British journal of psychiatry the journal of mental science 190, S. 293-298.

Lorant, Vincent/Deliège, Denise/Eaton, William W./Robert, A./Philippot, Pierre./Ansseau, Marc (2003): Socioeconomic Inequalities in Depression. A MetaAnalysis. In: American journal of epidemiology 157, 2, S. 98-112.

Lorgelly, Paula K./Lindley, Joanne (2008): What is the relationship between income inequality and health? Evidence from the BHPS. In: Health economics 17, 2, S. 249-265.

Lucumi, Diego I./Schulz, Amy J./Roux, Ana V. Diez/Grogan-Kaylor, Andrew (2017): Income inequality and high blood pressure in Colombia: a multilevel analysis. In: Cadernos de saude publica 33, 11, S. e00172316.

Lunney, June R./Lynn, Joanne/Foley, Daniel J./Lipson, Steven/Guralnik, Jack M. (2003): Patterns of functional decline at the end of life. In: JAMA 289, 18, S. $2387-$ 2392.

Luppa, M./Sikorski, C./Luck, T./Ehreke, L./Konnopka, A./Wiese, B./Weyerer, S./König, H-H/Riedel-Heller, S. G. (2012): Age- and gender-specific prevalence of depression in latest-life--systematic review and meta-analysis. In: Journal of Affective Disorders 136, 3, S. 212-221. 
Lynch, John W./Kaplan, George A. (1997): Understanding how inequality in the distribution of income affects health. In: Journal of Health Psychology 2, 3, S. 297-314.

Lynch, John W./Smith, George Davey/Harper, Sam/Hillemeier, Marianne/Ross, Nancy A./Kaplan, George A./Wolfson, Michael (2004): Is Income Inequality a Determinant of Population Health? Part 1. A Systematic Review. In: The Milbank Quarterly 82, 1, S. 5-99.

Lynch, John W./Smith, George Davey/Kaplan, George A./House, James S. (2000): Income inequality and mortality. Importance to health of individual income, psychosocial environment, or material conditions. In: BMJ 320, 7243, S. 1200-1204.

Mabughi, Nyiwul/Selim, Tarek (2006): Poverty as social deprivation. A survey. In: Review of Social Economy 64, 2, S. 181-204.

Mackenbach, Johan P. (2010): New trends in health inequalities research. Now it's personal. In: The Lancet 376, 9744, S. 854-855.

Madianos, Michael/Economou, Marina/Alexiou, Tatiana/Stefanis, Costas (2011): Depression and economic hardship across Greece in 2008 and 2009: two cross-sectional surveys nationwide. In: Social psychiatry and psychiatric epidemiology 46, 10, S. 943-952.

Maio, Fernando G. de (2008): Ecological analysis of the health effects of income inequality in Argentina. In: Public health 122, 5, S. 487-496.

Mani, Anandi/Mullainathan, Sendhil/Shafir, Eldar/Zhao, Jiaying (2013): Poverty impedes cognitive function. In: Science (New York, N.Y.) 341, 6149, S. 976-980.

Manini, Todd (2001): Development of physical disability in older adults. In: Current Aging Science 4, 3, S. 184-191.

Mansyur, Carol/Amick, Benjamin C./Harrist, Ronald B./Franzini, Luisa (2008): Social capital, income inequality, and self-rated health in 45 countries. In: Social science \& medicine (1982) 66, 1, S. 43-56.

Marmot, Michael (2004): Status syndrome. In: Significance 1, 4, S. 150-154.

Marmot, Michael/Ryff, Carol D./Bumpass, Larry L./Shipley, Martin/Marks, Nadine F. (1997): Social inequalities in health. Next questions and converging evidence. In: Social science \& medicine 44, 6, S. 901-910.

Marshall, Alan/Jivraj, Stephen/Nazroo, James/Tampubolon, Gindo/Vanhoutte, Bram (2014): Does the level of wealth inequality within an area influence the prevalence of depression amongst older people? In: Health \& place 27, S. 194-204.

Martikainen, Pekka/Valkonen, Tapani/Moustgaard, Heta (2009): The effects of individual taxable income, household taxable income, and household disposable income on mortality in Finland, 1998-2004. In: Population studies 63, 2, S. 147-162.

Martín-López, Rocío/Hernández-Barrera, Valentín/Andres, Ana Lopez de/Garrido, Pilar Carrasco/Miguel, Angel Gil de/García, Rodrigo Jiménez (2010): Breast and cervical cancer screening in Spain and predictors of adherence. In: European journal of cancer prevention the official journal of the European Cancer Prevention Organisation (ECP) 19, 3, S. 239-245.

Masson, Torsten/Leßmann, Ortrud (2016): Ökologisch nachhaltiger Konsum und ungleiche Teilhabe. In: Forschungsverbund sozioökonomische Berichterstattung (Hrsg.): Berichterstattung zur sozioökonomischen Entwicklung in Deutschland. Bielefeld: W. Bertelsmann Verlag. 
Matthews, Ruth J./Smith, Lucy K./Hancock, Ruth M./Jagger, Carol/Spiers, Nicola A. (2005): Socioeconomic factors associated with the onset of disability in older age: a longitudinal study of people aged 75 years and over. In: Social science \& medicine (1982) 61, 7, S. 1567-1575.

Matthews, Sharon/Stansfeld, Stephen/Power, Chris (1999): Social support at age 33. The influence of gender, employment status and social class. In: Social science \& medicine 49, 1, S. 133-142.

Mayhew, Kathryn P./Lempers, Jacques D. (1998): The Relation among Financial Strain, Parenting, Parent Self-Esteem, and Adolescent Self-Esteem. In: The Journal of Early Adolescence 18, 2, S. 145-172.

Mayraz, Guy/Wagner, Gert G./Schupp, Jürgen (2009): Life Satisfaction and Relative Income - Perceptions and Evidence. SOEPpapers on Multidisciplinary Panel Data Research. Berlin.

Mazzonna, Fabrizio/Peracchi, Franco (2017): Unhealthy Retirement? In: Journal of Human Resources 52, 1, S. 128-151.

McEwen, Bruce S. (1998): Protective and damaging effects of stress mediators. In: New England journal of medicine, 338.3, S. 171-179.

McKenzie, Sarah K./Imlach Gunasekara, Fiona/Richardson, Ken/Carter, Kristie (2014): Do changes in socioeconomic factors lead to changes in mental health? Findings from three waves of a population based panel study. In: Journal of epidemiology and community health 68,3, S. 253-260.

McLaughlin, K. A./Nandi, A./Keyes, K. M./Uddin, M./Aiello, A. E./Galea, S./Koenen, K. C. (2012): Home foreclosure and risk of psychiatric morbidity during the recent financial crisis. In: Psychological medicine 42, 7, S. 1441-1448.

McMunn, Anne/Nazroo, James/Breeze, Elizabeth (2009): Inequalities in health at older ages: a longitudinal investigation of the onset of illness and survival effects in England. In: Age and Ageing 38, 2, S. 181-187.

McPherson, Miller/Smith-Lovin, Lynn/Cook, James M. (2001): Birds of a feather: Homophily in social networks. In: Annual review of sociology 27, 1, S. 415-444.

Medeiros, Ian de (2017): Keeping up with the Joneses: Income inequality's effect on mental health. Doctoral dissertation. Oxford, Ohio: Miami University.

Melar, Natalia/Rossi, Máximo (2012): A Cross-Country Analysis of the Risk Factors for Depression at the Micro and Macro Levels. In: American Journal of Economics and Sociology 71, 2, S. 354-376.

Mellor, Jennifer M./Milyo, Jeffrey (2001): Reexamining the Evidence of an Ecological Association between Income Inequality and Health. In: Journal of Health Politics, Policy and Law 26, 3, S. 487-522.

Mellor, Jennifer M./Milyo, Jeffrey (2003): Is Exposure to Income Inequality a Public Health Concern? Lagged Effects of Income Inequality on Individual and Population Health. In: Health Services Research 38, 1p1, S. 137-151.

Meltzer, H./Bebbington, P./Brugha, T./Jenkins, R./McManus, S./Stansfeld, S. (2010): Job insecurity, socio-economic circumstances and depression. In: Psychological medicine 40, 8, S. 1401-1407.

Meltzer, Herbert Y. (1990): Role of Serotonin in Depression. In: Annals of the New York Academy of Sciences 600, 1 The Neurophar, S. 486-499. 
Messias, Erick/Eaton, William W./Grooms, Amy N. (2011): Income Inequality and Depression Prevalence Across the United States: An Ecological Study. In: Psychiatric Services 62, 7, S. 710-712.

Meyer, Bruce/Mok, Wallace K./Sullivan, James (2009): The Under-Reporting of Transfers in Household Surveys: Its Nature and Consequences. Cambridge, MA: National Bureau of Economic Research.

Mirowsky, John/Kim, Jinyoung (2007): Graphing Age Trajectories. Vector Graphs, Synthetic and Virtual Cohort Projections, and Cross-Sectional Profiles of Depression. In: Sociological Methods \& Research 35, 4, S. 497-541.

Mirowsky, John/Reynolds, John R. (2000): Age, Depression, and Attrition in the National Survey of Families and Households. In: Sociological Methods \& Research 28, 4, S. 476-504.

Mirowsky, John/Ross, Catherine E. (1992): Age and Depression. In: Journal of Health and Social Behavior 33, 3, S. 187-205.

Mirowsky, John/Ross, Catherine E. (2001): Age and the effect of economic hardship on depression. In: Journal of Health and Social Behavior 42, S. 132-150.

Mirowsky, John/Ross, Catherine E. (2003): Social causes of psychological distress. 2. ed. Social institutions and social change. Hawthorne, NY: DE GRUYTER.

Mirowsky, John/Ross, Catherine E. (2010): Well-Being across the Life Course. In: Scheid, T. L./Brown, T. N. (Hrsg.): A handbook for the study of mental health. Social contexts, theories, and systems. 2nd ed. Cambridge, New York: Cambridge University Press, S. 361-383.

MISSOC (2018): Missoc database - Comparative tables. https://www.missoc.org/ [Zugriff: 07.08.2018].

Monserud, Maria A./Markides, Kyriakos S. (2017): Changes in depressive symptoms during widowhood among older Mexican Americans: the role of financial strain, social support, and church attendance. In: Aging \& Mental Health 21, 6, S. 586594.

Montez, Jennifer Karas/Hayward, Mark D./Wolf, Douglas A. (2017): Do U.S. states' socioeconomic and policy contexts shape adult disability? In: Social science \& medicine (1982) 178, S. 115-126.

Mood, C. (2010): Logistic Regression. Why We Cannot Do What We Think We Can Do, and What We Can Do About It. In: European Sociological Review 26, 1, S. 67-82.

Moore, Alison A./Hays, Ron D./Greendale, Gail A./Damesyn, Mark/Reuben, David B. (1999): Drinking Habits Among Older Persons. Findings from the NHANES I Epidemiologic Follow-up Study (1982-84). In: Journal of the American Geriatrics Society 47, 4, S. 412-416.

Moore, Jeffrey C./Stinson, Linda L./Welniak, Edward J. (2000): Income Measurement Error in Surveys: A Review. In: Journal of Official Statistics 16, 4, S. 331-361.

Moret, Chantal/Briley, Mike (2011): The importance of norepinephrine in depression. In: Neuropsychiatric disease and treatment 7, Suppl 1, S. 9-13.

Morris, J. N. (2000): A minimum income for healthy living. In: Journal of Epidemiology \& Community Health 54, 12, S. 885-889. 
Morris, J. N./Wilkinson, Paul/Dangour, Alan D./Deeming, Christopher/Fletcher, Astrid (2007): Defining a minimum income for healthy living (MIHL): older age, England. In: International journal of epidemiology 36, 6, S. 1300-1307.

Morris, Matthew C./Rao, Uma/Garber, Judy (2012): Cortisol responses to psychosocial stress predict depression trajectories: social-evaluative threat and prior depressive episodes as moderators. In: Journal of Affective Disorders 143, 1-3, S. 223-230.

Mottram, Sara/Peat, George/Thomas, Elaine/Wilkie, Ross/Croft, Peter (2008): Patterns of pain and mobility limitation in older people: cross-sectional findings from a population survey of 18,497 adults aged 50 years and over. In: Quality of life research an international journal of quality of life aspects of treatment, care and rehabilitation 17, 4, S. 529-539.

Muller, J. E./Tofler, G. H./Stone, P. H. (1989): Circadian variation and triggers of onset of acute cardiovascular disease. In: Circulation, 79, S. 733-743.

Müller, Walter/Brauns, Hildegard/Steinmann, Susanne (2002): Expansion und Erträge tertiärer Bildung in Deutschland, Frankreich und im Vereinigten Königreich. In: Berliner Journal für Soziologie 12, 1, S. 37-62.

Muramatsu, Naoko (2003): County-Level Income Inequality and Depression among Older Americans. In: Health Services Research 38, 6p2, S. 1863-1884.

Murray, Christopher J. L./Barber, Ryan M./Foreman, Kyle J./Ozgoren, Ayse Abbasoglu/Abd-Allah, Foad/Abera, Semaw F./Aboyans, Victor/Abraham, Jerry P./Abubakar, Ibrahim/Abu-Raddad, Laith J./Abu-Rmeileh, Niveen M./Achoki, Tom/Ackerman, Ilana N./Ademi, Zanfina/Adou, Arsène K./Adsuar, José C./Afshin, Ashkan/Agardh, Emilie E./Alam, Sayed Saidul/Alasfoor, Deena/Albittar, Mohammed I./Alegretti, Miguel A./Alemu, Zewdie A./Alfonso-Cristancho, Rafael/Alhabib, Samia/Ali, Raghib/Alla, François/Allebeck, Peter/Almazroa, Mohammad A./Alsharif, Ubai/Alvarez, Elena/Alvis-Guzman, Nelson/Amare, Azmeraw T./Ameh, Emmanuel A./Amini, Heresh/Ammar, Walid/Anderson, H. Ross/Anderson, Benjamin O./Antonio, Carl Abelardo T./Anwari, Palwasha/Arnlöv, Johan/Arsenijevic, Valentina S. Arsic/Artaman, Al/Asghar, Rana J./Assadi, Reza/Atkins, Lydia S./Avila, Marco A./Awuah, Baffour/Bachman, Victoria F./Badawi, Alaa/Bahit, Maria C./Balakrishnan, Kalpana/Banerjee, Amitava/Barker-Collo, Suzanne L./Barquera, Simon/Barregard, Lars/Barrero, Lope H./Basu, Arindam/Basu, Sanjay/Basulaiman, Mohammed O./Beardsley, Justin/Bedi, Neeraj/Beghi, Ettore/Bekele, Tolesa/Bell, Michelle L./Benjet, Corina/Bennett, Derrick A./Bensenor, Isabela M./Benzian, Habib/Bernabé, Eduardo/Bertozzi-Villa, Amelia/Beyene, Tariku J./Bhala, Neeraj/Bhalla, Ashish/Bhutta, Zulfiqar A./Bienhoff, Kelly/Bikbov, Boris/Biryukov, Stan/Blore, Jed D./Blosser, Christopher D./Blyth, Fiona M./Bohensky, Megan A./Bolliger, Ian W./Başara, Berrak Bora/Bornstein, Natan M./Bose, Dipan/Boufous, Soufiane/Bourne, Rupert R. A./Boyers, Lindsay N./Brainin, Michael/Brayne, Carol E./Brazinova, Alexandra/Breitborde, Nicholas J. K./Brenner, Hermann/Briggs, Adam D./Brooks, Peter M./Brown, Jonathan C./Brugha, Traolach S./Buchbinder, Rachelle/Buckle, Geoffrey C./Budke, Christine M./Bulchis, Anne/Bulloch, Andrew G./Campos-Nonato, Ismael R./Carabin, Hélène/Carapetis, Jonathan R./Cárdenas, Rosario/Carpenter, David O./Caso, Valeria/Castañeda-Orjuela, Carlos A./Castro, Ruben E./Catalá-López, Ferrán/Cavalleri, Fiorella/Çavlin, 
Alanur/Chadha, Vineet K./Chang, Jung-Chen/Charlson, Fiona J./Chen, Honglei/Chen, Wanqing/Chiang, Peggy P./Chimed-Ochir, Odgerel/Chowdhury, Rajiv/Christensen, Hanne/Christophi, Costas A./Cirillo, Massimo/Coates, Matthew M./Coffeng, Luc E./Coggeshall, Megan S./Colistro, Valentina/Colquhoun, Samantha M./Cooke, Graham S./Cooper, Cyrus/Cooper, Leslie T./Coppola, Luis M./Cortinovis, Monica/Criqui, Michael H./Crump, John A./Cuevas-Nasu, Lucia/Danawi, Hadi/Dandona, Lalit/Dandona, Rakhi/Dansereau, Emily/Dargan, Paul I./Davey, Gail/Davis, Adrian/Davitoiu, Dragos V./Dayama, Anand/Leo, Diego de/Degenhardt, Louisa/Del Pozo-Cruz, Borja/Dellavalle, Robert P./Deribe, Kebede/Derrett, Sarah/Des Jarlais, Don C./Dessalegn, Muluken/Dharmaratne, Samath D./Dherani, Mukesh K./Diaz-Torné, Cesar/Dicker, Daniel/Ding, Eric L./Dokova, Klara/Dorsey, E. Ray/Driscoll, Tim R./Duan, Leilei/Duber, Herbert C./Ebel, Beth E./Edmond, Karen M./Elshrek, Yousef M./Endres, Matthias/Ermakov, Sergey P./Erskine, Holly E./Eshrati, Babak/Esteghamati, Alireza/Estep, Kara/Faraon, Emerito Jose A./Farzadfar, Farshad/Fay, Derek F./Feigin, Valery L./Felson, David T./Fereshtehnejad, Seyed-Mohammad/Fernandes, Jefferson G./Ferrari, Alize J./Fitzmaurice, Christina/Flaxman, Abraham D./Fleming, Thomas D./Foigt, Nataliya/Forouzanfar, Mohammad H./Fowkes, F. Gerry R./Paleo, Urbano Fra./Franklin, Richard C./Fürst, Thomas/Gabbe, Belinda/Gaffikin, Lynne/Gankpé, Fortuné G./Geleijnse, Johanna M./Gessner, Bradford D./Gething, Peter/Gibney, Katherine B./Giroud, Maurice/Giussani, Giorgia/Dantes, Hector Gomez/Gona, Philimon/González-Medina, Diego/Gosselin, Richard A./Gotay, Carolyn C./Goto, Atsushi/Gouda, Hebe N./Graetz, Nicholas/Gugnani, Harish C./Gupta, Rahul/Gupta, Rajeev/Gutiérrez, Reyna A./Haagsma, Juanita/Hafezi-Nejad, Nima/Hagan, Holly/Halasa, Yara A./Hamadeh, Randah R./Hamavid, Hannah/Hammami, Mouhanad/Hancock, Jamie/Hankey, Graeme J./Hansen, Gillian M./Hao, Yuantao/Harb, Hilda L./Haro, Josep Maria/Havmoeller, Rasmus/Hay, Simon I./Hay, Roderick J./Heredia-Pi, Ileana B./Heuton, Kyle R./Heydarpour, Pouria/Higashi, Hideki/Hijar, Martha/Hoek, Hans W./Hoffman, Howard J./Hosgood, H. Dean/Hossain, Mazeda/Hotez, Peter J./Hoy, Damian G./Hsairi, Mohamed/Hu, Guoqing/Huang, Cheng/Huang, John J./Husseini, Abdullatif/Huynh, Chantal/Iannarone, Marissa L./Iburg, Kim M./Innos, Kaire/Inoue, Manami/Islami, Farhad/Jacobsen, Kathryn H./Jarvis, Deborah L./Jassal, Simerjot K./Jee, Sun Ha/Jeemon, Panniyammakal/Jensen, Paul N./Jha, Vivekanand/Jiang, Guohong/Jiang, Ying/Jonas, Jost B./Juel, Knud/Kan, Haidong/Karch, André/Karema, Corine K./Karimkhani, Chante/Karthikeyan, Ganesan/Kassebaum, Nicholas J./Kaul, Anil/Kawakami, Norito/Kazanjan, Konstantin/Kemp, Andrew H./Kengne, Andre P./Keren, Andre/Khader, Yousef S./Khalifa, Shams Eldin A./Khan, Ejaz A./Khan, Gulfaraz/Khang, Young-Ho/Kieling, Christian/Kim, Daniel/Kim, Sungroul/Kim, Yunjin/Kinfu, Yohannes/Kinge, Jonas M./Kivipelto, Miia/Knibbs, Luke D./Knudsen, Ann Kristin/Kokubo, Yoshihiro/Kosen, Soewarta/Krishnaswami, Sanjay/Defo, Barthelemy Kuate/Bicer, Burcu Kucuk/Kuipers, Ernst J./Kulkarni, Chanda/Kulkarni, Veena S./Kumar, G. Anil/Kyu, Hmwe H./Lai, Taavi/Lalloo, Ratilal/Lallukka, Tea/Lam, Hilton/Lan, Qing/van Lansingh, C./Larsson, Anders/Lawrynowicz, Alicia E. B./Leasher, Janet L./Leigh, James/Leung, Ricky/Levitz, Carly E./Li, Bin/Li, Yichong/Li, 
Yongmei/Lim, Stephen S./Lind, Maggie/Lipshultz, Steven E./Liu, Shiwei/Liu, Yang/Lloyd, Belinda K./Lofgren, Katherine T./Logroscino, Giancarlo/Looker, Katharine J./Lortet-Tieulent, Joannie/Lotufo, Paulo A./Lozano, Rafael/Lucas, Robyn M./Lunevicius, Raimundas/Lyons, Ronan A./Ma, Stefan/Macintyre, Michael F./Mackay, Mark T./Majdan, Marek/Malekzadeh, Reza/Marcenes, Wagner/Margolis, David J./Margono, Christopher/Marzan, Melvin B./Masci, Joseph R./Mashal, Mohammad T./Matzopoulos, Richard/Mayosi, Bongani M./Mazorodze, Tasara T./Mcgill, Neil W./Mcgrath, John J./McKee, Martin/Mclain, Abigail/Meaney, Peter A./Medina, Catalina/Mehndiratta, Man Mohan/Mekonnen, Wubegzier/Melaku, Yohannes A./Meltzer, Michele/Memish, Ziad A./Mensah, George A./Meretoja, Atte/Mhimbira, Francis A./Micha, Renata/Miller, Ted R./Mills, Edward J./Mitchell, Philip B./Mock, Charles N./Ibrahim, Norlinah Mohamed/Mohammad, Karzan A./Mokdad, Ali H./Mola, Glen L. D./Monasta, Lorenzo/Hernandez, Julio C. Montañez/Montico, Marcella/Montine, Thomas J./Mooney, Meghan D./Moore, Ami R./Moradi-Lakeh, Maziar/Moran, Andrew E./Mori, Rintaro/Moschandreas, Joanna/Moturi, Wilkister N./Moyer, Madeline L./Mozaffarian, Dariush/Msemburi, William T./Mueller, Ulrich O./Mukaigawara, Mitsuru/Mullany, Erin C./Murdoch, Michele E./Murray, Joseph/Murthy, Kinnari S./Naghavi, Mohsen/Naheed, Aliya/Naidoo, Kovin S./Naldi, Luigi/Nand, Devina/Nangia, Vinay/Narayan, K. M. Venkat/Nejjari, Chakib/Neupane, Sudan P./Newton, Charles R./Ng, Marie/Ngalesoni, Frida N./Nguyen, Grant/Nisar, Muhammad I./Nolte, Sandra/Norheim, Ole F./Norman, Rosana E./Norrving, Bo/Nyakarahuka, Luke/Oh, In-Hwan/Ohkubo, Takayoshi/Ohno, Summer L./Olusanya, Bolajoko O./Opio, John Nelson/Ortblad, Katrina/Ortiz, Alberto/Pain, Amanda W./Pandian, Jeyaraj D./Panelo, Carlo Irwin A./Papachristou, Christina/Park, Eun-Kee/Park, Jae-Hyun/Patten, Scott B./Patton, George C./Paul, Vinod K./Pavlin, Boris I./Pearce, Neil/Pereira, David M./Perez-Padilla, Rogelio/PerezRuiz, Fernando/Perico, Norberto/Pervaiz, Aslam/Pesudovs, Konrad/Peterson, Carrie B./Petzold, Max/Phillips, Michael R./Phillips, Bryan K./Phillips, David E./Piel, Frédéric B./Plass, Dietrich/Poenaru, Dan/Polinder, Suzanne/Pope, Daniel/Popova, Svetlana/Poulton, Richie G./Pourmalek, Farshad/Prabhakaran, Dorairaj/Prasad, Noela M./Pullan, Rachel L./Qato, Dima M./Quistberg, D. Alex/Rafay, Anwar/Rahimi, Kazem/Rahman, Sajjad U./Raju, Murugesan/Rana, Saleem M./Razavi, Homie/Reddy, K. Srinath/Refaat, Amany/Remuzzi, Giuseppe/Resnikoff, Serge/Ribeiro, Antonio L./Richardson, Lee/Richardus, Jan Hendrik/Roberts, D. Allen/Rojas-Rueda, David/Ronfani, Luca/Roth, Gregory A./Rothenbacher, Dietrich/Rothstein, David H./Rowley, Jane T./Roy, Nobhojit/Ruhago, George M./Saeedi, Mohammad Y./Saha, Sukanta/Sahraian, Mohammad Ali/Sampson, Uchechukwu K. A./Sanabria, Juan R./Sandar, Logan/Santos, Itamar S./Satpathy, Maheswar/Sawhney, Monika/Scarborough, Peter/Schneider, Ione J./Schöttker, Ben/Schumacher, Austin E./Schwebel, David C./Scott, James G./Seedat, Soraya/Sepanlou, Sadaf G./Serina, Peter T./Servan-Mori, Edson E./Shackelford, Katya A./Shaheen, Amira/Shahraz, Saeid/Levy, Teresa Shamah/Shangguan, Siyi/She, Jun/Sheikhbahaei, Sara/Shi, Peilin/Shibuya, Kenji/Shinohara, Yukito/Shiri, Rahman/Shishani, Kawkab/Shiue, Ivy/Shrime, Mark G./Sigfusdot- 
tir, Inga D./Silberberg, Donald H./Simard, Edgar P./Sindi, Shireen/Singh, Abhishek/Singh, Jasvinder A./Singh, Lavanya/Skirbekk, Vegard/Slepak, Erica Leigh/Sliwa, Karen/Soneji, Samir/Søreide, Kjetil/Soshnikov, Sergey/Sposato, Luciano A./Sreeramareddy, Chandrashekhar T./Stanaway, Jeffrey D./Stathopoulou, Vasiliki/Stein, Dan J./Stein, Murray B./Steiner, Caitlyn/Steiner, Timothy J./Stevens, Antony/Stewart, Andrea/Stovner, Lars J./Stroumpoulis, Konstantinos/Sunguya, Bruno F./Swaminathan, Soumya/Swaroop, Mamta/Sykes, Bryan L./Tabb, Karen M./Takahashi, Ken/Tandon, Nikhil/Tanne, David/Tanner, Marcel/Tavakkoli, Mohammad/Taylor, Hugh R./Ao, Braden J. Te/Tediosi, Fabrizio/Temesgen, Awoke M./Templin, Tara/Have, Margreet ten/Tenkorang, Eric Y./Terkawi, Abdullah S./Thomson, Blake/Thorne-Lyman, Andrew L./Thrift, Amanda G./Thurston, George D./Tillmann, Taavi/Tonelli, Marcello/Topouzis, Fotis/Toyoshima, Hideaki/Traebert, Jefferson/Tran, Bach X./Trillini, Matias/Truelsen, Thomas/Tsilimbaris, Miltiadis/Tuzcu, Emin M./Uchendu, Uche S./Ukwaja, Kingsley N./Undurraga, Eduardo A./Uzun, Selen B./van Brakel, Wim H./van de Vijver, Steven/van Gool, Coen H./van Os, Jim/Vasankari, Tommi J./Venketasubramanian, N./Violante, Francesco S./Vlassov, Vasiliy V./Vollset, Stein Emil/Wagner, Gregory R./Wagner, Joseph/Waller, Stephen G./Wan, Xia/Wang, Haidong/Wang, Jianli/Wang, Linhong/Warouw, Tati S./Weichenthal, Scott/Weiderpass, Elisabete/Weintraub, Robert G./Wenzhi, Wang/Werdecker, Andrea/Westerman, Ronny/Whiteford, Harvey A./Wilkinson, James D./Williams, Thomas N./Wolfe, Charles D./Wolock, Timothy M./Woolf, Anthony D./Wulf, Sa$\mathrm{rah} /$ Wurtz, Brittany/Xu, Gelin/Yan, Lijing L./Yano, Yuichiro/Ye, Pengpeng/Yentür, Gökalp K./Yip, Paul/Yonemoto, Naohiro/Yoon, SeokJun/Younis, Mustafa Z./Yu, Chuanhua/Zaki, Maysaa E./Zhao, Yong/Zheng, Yingfeng/Zonies, David/Zou, Xiaonong/Salomon, Joshua A./Lopez, Alan D./Vos, Theo (2015): Global, regional, and national disability-adjusted life years (DALYs) for 306 diseases and injuries and healthy life expectancy (HALE) for 188 countries, 1990-2013. Quantifying the epidemiological transition. In: The Lancet 386, 10009, S. 2145-2191.

Musterd, Sako (2016): Social and Ethnic Segregation in Europe. Levels, Causes, and Effects. In: Journal of Urban Affairs 27, 3, S. 331-348.

Nagi, Saad Z. (1991): Disability concepts revisted: implications for prevention. In: Pope, A. M./Tarlov, A. R. (Hrsg.): Disability in America: Toward a National Agenda for Prevention. Washington, D.C.: National Academy Press, S. 309-327.

Nepon, Taryn/Flett, Gordon L./Hewitt, Paul L./Molnar, Danielle S. (2011): Perfectionism, negative social feedback, and interpersonal rumination in depression and social anxiety. In: Canadian Journal of Behavioural Science/Revue canadienne des sciences du comportement 43, 4, S. 297-308.

Netuveli, Gopalakrishnan/Wiggins, Richard D./Hildon, Zoe/Montgomery, Scott M./Blane, David (2006): Quality of life at older ages: evidence from the English longitudinal study of aging (wave 1). In: Journal of epidemiology and community health 60,4 , S. 357-363.

Neumann, Frieder (2015): Soziale Mindestsicherung in Europa - Effektivität und soziale Gerechtigkeit in der EU-27 im Vergleich. In: Zeitschrift für Staats- und Europawissenschaften 13, 3, S. 420-449. 
Neurath, Otto (1937): Inventory of the Standard of Living. In: Zeitschrift für Sozialforschung 6,1 , S. 140-151.

Newman, A. B./Kupelian, V./Visser, M./Simonsick, E. M./Goodpaster, B. H./Kritchevsky, S. B./Tylavsky, F. A./Rubin, S. M./Harris, T. B. (2006): Strength, But Not Muscle Mass, Is Associated With Mortality in the Health, Aging and Body Composition Study Cohort. In: The Journals of Gerontology Series A: Biological Sciences and Medical Sciences 61, 1, S. 72-77.

Nicklett, Emily J./Kadell, Andria R. (2013): Fruit and vegetable intake among older adults: a scoping review. In: Maturitas 75, 4, S. 305-312.

Niehues, Judith (2016): Ungleichheit. Wahrnehmung und Wirklichkeit - ein internationaler Vergleich. In: Wirtschaftsdienst 96, S1, S. 13-18.

Nisslé, Sonja/Bschor, Tom (2002): Winning the jackpot and depression: Money cannot buy happiness. In: International journal of psychiatry in clinical practice 6,3 , S. $183-186$.

Nolan, Brian/Whelan, Christopher T. (2010): Using non-monetary deprivation indicators to analyze poverty and social exclusion. Lessons from Europe? In: Journal of Policy Analysis and Management 29, 2, S. 305-325.

Nolan, Brian/Whelan, Christopher T. (2011): Poverty and Deprivation in Europe. Oxford University Press.

Nowatzki, Nadine R. (2012): Wealth inequality and health: a political economy perspective. In: International journal of health services planning, administration, evaluation 42, 3, S. 403-424.

Nutt, David J. (2008): Relationship of neurotransmitters to the symptoms of major depressive disorder. In: The Journal of Clinical Psychiatry 69, Suppl E1, S. 4-7.

OECD (2016): Income Distribution and Poverty.

OECD (2018a): Health resources (indicator). OECD.

OECD (2018b): Level of GDP per capita and productivity. https://stats.oecd.org/Index.aspx?DataSetCode=PDB_LV [Zugriff: 02.02.2018].

Oliver, J. Eric (1999): The Effects of Metropolitan Economic Segregation on Local Civic Participation. In: American Journal of Political Science 43, 1, S. 186.

Olsen, Karen M./Dahl, Svenn-Age (2007): Health differences between European countries. In: Social science \& medicine (1982) 64, 8, S. 1665-1678.

O'Neill, Barbara/Prawitz, Aimee/Sorhaindo, Benoit/Kim, Jinhee/Garman, E. Thomas (2006): Changes in Health, Negative Financial Events, and Financial Distress/Financial Well-Being for Debt Management Program Clients. In: Journal of Financial Counseling and Planning 17, 2, S. 46-63.

Ormel, J./Oldehinkel, A. J./Brilman, E. I. (2001): The interplay and etiological continuity of neuroticism, difficulties, and life events in the etiology of major and subsyndromal, first and recurrent depressive episodes in later life. In: The American Journal of Psychiatry 158, 6, S. 885-891.

O'Sullivan, K. C./Howden-Chapman, P. L./Fougere, G. (2012): Death by disconnection. The missing public health voice in newspaper coverage of a fuel povertyrelated death. In: Kotuitui: New Zealand Journal of Social Sciences Online 7, 1, S. 51-60. 
Pabayo, Roman/Kawachi, Ichiro/Gilman, Stephen E. (2014): Income inequality among American states and the incidence of major depression. In: Journal of epidemiology and community health 68, 2, S. 110-115.

Pabayo, Roman/Kawachi, Ichiro/Gilman, Stephen E. (2015): US State-level income inequality and risks of heart attack and coronary risk behaviors: longitudinal findings. In: International journal of public health 60,5 , S. 573-588.

Palta, Priya/Szanton, Sarah L./Semba, Richard D./Thorpe, Roland J./Varadhan, Ravi/Fried, Linda P. (2015): Financial strain is associated with increased oxidative stress levels: the Women's Health and Aging Studies. In: Geriatric nursing (New York, N.Y.) 36, 2 Suppl, S. S33-7.

Papp, Lauren M./Cummings, E. Mark/Goeke-Morey, Marcie C. (2009): For Richer, for Poorer: Money as a Topic of Marital Conflict in the Home. In: Family relations 58, 1, S. 91-103.

Park, Bo Hyun/Jung, Minsoo/Lee, Tae Jin (2009): Associations of income and wealth with health status in the Korean elderly. In: Journal of preventive medicine and public health = Yebang Uihakhoe chi 42, 5, S. 275-282.

Paskov, Marii/Dewilde, Caroline (2012): Income inequality and solidarity in Europe. In: Research in Social Stratification and Mobility 30, 4, S. 415-432.

Patel, Vikram/Burns, Jonathan K./Dhingra, Monisha/Tarver, Leslie/Kohrt, Brandon A./Lund, Crick (2018): Income inequality and depression: a systematic review and meta-analysis of the association and a scoping review of mechanisms. In: World psychiatry official journal of the World Psychiatric Association (WPA) 17, 1, S. 76-89.

Patten, Scott B. (2013): Major depression epidemiology from a diathesis-stress conceptualization. In: BMC psychiatry 13, S. 19.

Peeters, Anna/Bonneux, Luc/Nusselder, Wilma J./Laet, Chris de/Barendregt, Jan J. (2004): Adult obesity and the burden of disability throughout life. In: Obesity research 12,7, S. 1145-1151.

Peeters, Geeske/Dobson, Annette J./Deeg, Dorly J. H./Brown, Wendy J. (2013): A lifecourse perspective on physical functioning in women. In: Bulletin of the World Health Organization 91, 9, S. 661-670.

Penninx, Brenda W.J.H. (1998): Depressive Symptoms and Physical Decline in Community-Dwelling Older Persons. In: JAMA 279, 21, S. 1720.

Pérez, Claudio E. (2002): Fruit and vegetable consumption. In: Health Reports 13, 3, S. 23-31.

Perrig-Chiello, Pasqualina/Hutchison, Sara (2010): Health and well-being in old age: the pertinence of a gender mainstreaming approach in research. In: Gerontology 56,2 , S. 208-213.

Pfoertner, Timo-Kolja/Andress, Hans-Juergen/Janssen, Christian (2011): Income or living standard and health in Germany: different ways of measurement of relative poverty with regard to self-rated health. In: International journal of public health 56,4 , S. 373-384.

Phongsavan, Philayrath/Chey, Tien/Bauman, Adrian/Brooks, Robert/Silove, Derrick (2006): Social capital, socio-economic status and psychological distress among Australian adults. In: Social science \& medicine (1982) 63, 10, S. 2546-2561. 
Piachaud, David (1992): Wie Misst Man Armut? In: Leibfried, S./Voges, W. (Hrsg.): Armut im modernen Wohlfahrtsstaat. Zeitschrift für Soziologie und Sozialpsychologie (Sonderhefte), Band 32. Wiesbaden: VS Verlag für Sozialwissenschaften, S. 63-87.

Pickett, Kate E./James, Oliver W./Wilkinson, Richard G. (2006): Income inequality and the prevalence of mental illness: a preliminary international analysis. In: Journal of epidemiology and community health 60, 7, S. 646-647.

Pickett, Kate E./Kelly, Shona/Brunner, Eric/Lobstein, Tim/Wilkinson, Richard G. (2005): Wider income gaps, wider waistbands? An ecological study of obesity and income inequality. In: Journal of epidemiology and community health 59, 8 , S. $670-674$.

Pickett, Kate E./Mookherjee, Jessica/Wilkinson, Richard G. (2005): Adolescent birth rates, total homicides, and income inequality in rich countries. In: American journal of public health 95, 7, S. 1181-1183.

Pickett, Kate E./Wilkinson, Richard G. (2010): Inequality: an underacknowledged source of mental illness and distress. In: The British journal of psychiatry the journal of mental science 197, 6, S. 426-428.

Pickett, Kate E./Wilkinson, Richard G. (2015): Income inequality and health: a causal review. In: Social science \& medicine (1982) 128, S. 316-326.

Pieper, L./Schulz, H./Klotsche, J./Eichler, T./Wittchen, H-U (2008): Depression als komorbide Störung in der primärärztlichen Versorgung. In: Bundesgesundheitsblatt, Gesundheitsforschung, Gesundheitsschutz 51, 4, S. 411-421.

Pignone, Michael P./Ammerman, Alice/Fernandez, Louise/Orleans, C.Tracy/Pender, Nola/Woolf, Steven/Lohr, Kathleen N./Sutton, Sonya (2003): Counseling to promote a healthy diet in adults. In: American Journal of Preventive Medicine 24, 1 , S. 75-92.

Pillmann, F. (2001): Sozialer Rang und Depression--ein Beispiel „,evolutionärer Psychopathologie“. In: Fortschritte der Neurologie-Psychiatrie 69, 6, S. 268-277.

Pinquart, Martin (2001): Correlates of subjective health in older adults. A meta-analysis. In: Psychology and Aging 16, 3, S. 414-426.

Piumatti, Giovanni (2017): Relations between longitudinal trajectories of subjective financial wellbeing with self-rated health among elderly. In: Medicina (Kaunas, Lithuania) 53, 5, S. 323-330.

Poortinga, Wouter (2006): Social capital: an individual or collective resource for health? In: Social science \& medicine (1982) 62, 2, S. 292-302.

Pop, Ioana Andreea/van Ingen, Erik/van Oorschot, Wim (2013): Inequality, Wealth and Health. Is Decreasing Income Inequality the Key to Create Healthier Societies? In: Social Indicators Research 113, 3, S. 1025-1043.

Porru, Fabio/Burdorf, Alex/Robroek, Suzan J. W. (2018): The impact of depressive symptoms on exit from paid employment in Europe: a longitudinal study with 4 years follow-up. In: European journal of public health.

Post, Robert M. (1992): Transduction of psychosocial stress into the neurobiology of recurrent affective disorder. In: The American Journal of Psychiatry 149, 8, S. 999-1010.

Power, Chris/Matthews, Sharon (1997): Origins of health inequalities in a national population sample. In: The Lancet 350, 9091, S. 1584-1589. 
Präg, Patrick/Mills, Melinda/Wittek, Rafael (2014): Income and Income Inequality as Social Determinants of Health. Do Social Comparisons Play a Role? In: European Sociological Review 30, 2, S. 218-229.

Prawitz, Aimee D./Kalkowski, Julie C./Cohart, Judith (2013): Responses to Economic Pressure by Low-Income Families. Financial Distress and Hopefulness. In: Journal of Family and Economic Issues 34, 1, S. 29-40.

Preston, Samuel H. (2007): The changing relation between mortality and level of economic development. Population Studies, Vol. 29, No. 2, July 1975. In: International journal of epidemiology 36, 3, S. 484-490.

Prince, M. J./Reischies, F./Beekman, A. T. F./Fuhrer, R./Jonker, C./Kivela, S.-L./Lawlor, B. A./Lobo, A./Magnusson, H./Fichter, M./van Oyen, H./Roelands, M./Skoog, I./Turrina, C./Copeland, J. R. M. (1999): Development of the EURO-D scale - a European Union initiative to compare symptoms of depression in 14 European centres. In: British Journal of Psychiatry 174, 04, S. 330-338.

Provençal, Nadine/Binder, Elisabeth B. (2015): The effects of early life stress on the epigenome: From the womb to adulthood and even before. In: Experimental neurology 268, S. 10-20.

Pudrovska, Tetyana/Anikputa, Benedicta (2014): Early-life socioeconomic status and mortality in later life: an integration of four life-course mechanisms. In: The journals of gerontology. Series B, Psychological sciences and social sciences 69, 3, S. $451-460$.

Pulkki, Laura/Kivimäki, Mika/Keltikangas-Järvinen, Liisa/Elovainio, Marko/Leino, Marketta/Viikari, Jorma (2003): Contribution of adolescent and early adult personality to the inverse association between education and cardiovascular risk behaviours. Prospective population-based cohort study. In: International journal of epidemiology 32, 6, S. 968-975.

Qi, Yaqiang (2012): The impact of income inequality on self-rated general health. Evidence from a cross-national study. In: Research in Social Stratification and Mobility 30,4 , S. $451-471$.

Radloff, Lenore Sawyer (2016): The CES-D Scale. In: Applied Psychological Measurement 1, 3, S. 385-401.

Rajan, Keertichandra/Kennedy, Jonathan/King, Lawrence (2013): Is wealthier always healthier in poor countries? The health implications of income, inequality, poverty, and literacy in India. In: Social science \& medicine (1982) 88, S. 98-107.

Rantanen, T./Masaki, K./Foley, D./Izmirlian, G./White, L./Guralnik, J. M. (1998): Grip strength changes over $27 \mathrm{yr}$ in Japanese-American men. In: Journal of applied physiology (Bethesda, Md. 1985) 85, 6, S. 2047-2053.

Rantanen, Taina (1999): Midlife Hand Grip Strength as a Predictor of Old Age Disability. In: JAMA 281, 6, S. 558.

Rapp, Ingmar/Stauder, Johannes (2019): Mental and Physical Health in Couple Relationships. Is It Better to Live Together? In: European Sociological Review 127, S. 171.

Raschke, Christian (2015): Unexpected Windfalls, Education, and Mental Health: Evidence from Lottery Winners in Germany. 
Rasella, Davide/Aquino, Rosana/Barreto, Mauricio Lima (2013): Impact of income inequality on life expectancy in a highly unequal developing country: the case of Brazil. In: Journal of epidemiology and community health 67, 8, S. 661-666.

Read, John (2010): Can poverty drive you mad? 'Schizophrenia', socio-economic status and the case for primary prevention. In: New Zealand Journal of Psychology 39, 2, S. 7-19.

Rebeira, Mayvis/Grootendorst, Paul/Coyte, Peter C./Aguirregabiria, Victor (2017): Does rising income inequality affect mortality rates in advanced economies? In: Economics: The Open-Access, Open-Assessment E-Journal.

Regidor, Enrique/Vallejo, Fernando/Giráldez-García, Carolina/Ortega, Paloma/Santos, Juana M./Astasio, Paloma/La Fuente, Luis de (2015): Low mortality in the poorest areas of Spain: adults residing in provinces with lower per capita income have the lowest mortality. In: European journal of epidemiology 30, 8, S. 637-648.

Reinberg, Alexander/Hummel, Markus (2007): Schwierige Fortschreibung: Der Trend bleibt - Geringqualifizierte sind häufiger arbeitslos. IAB-Kurzbericht. Nürnberg.

Ribeiro, Wagner Silva/Bauer, Annette/Andrade, Mário César Rezende/York-Smith, Marianna/Pan, Pedro Mario/Pingani, Luca/Knapp, Martin/Coutinho, Evandro Silva Freire/Evans-Lacko, Sara (2017): Income inequality and mental illness-related morbidity and resilience. A systematic review and meta-analysis. In: The Lancet Psychiatry 4, 7, S. 554-562.

Rice, Neil E./Lang, Iain A./Henley, William/Melzer, David (2011): Common health predictors of early retirement: findings from the English Longitudinal Study of Ageing. In: Age and Ageing 40, 1, S. 54-61.

Richardson, Thomas/Elliott, Peter/Roberts, Ronald (2013): The relationship between personal unsecured debt and mental and physical health: a systematic review and meta-analysis. In: Clinical psychology review 33, 8, S. 1148-1162.

Robine, Jean-Marie/Jagger, Carol/The Euro-Reves Group (2003): Creating a coherent set of indicators to monitor health across Europe. The Euro-REVES 2 project. In: The European Journal of Public Health 13, suppl 3, S. 6-14.

Rodgers, G. B. (1979): Income and inequality as determinants of mortality: an international cross-section analysisgrav. In: Population studies 33, 2, S. 343-351.

Roh, Myoung-Sun/Jeon, Hong Jin/Kim, Hana/Han, Sung Koo/Hahm, Bong-Jin (2010): The prevalence and impact of depression among medical students: a nationwide cross-sectional study in South Korea. In: Academic medicine journal of the Association of American Medical Colleges 85, 8, S. 1384-1390.

Rohde, Nicholas/Tang, K. K./Osberg, Lars/Rao, Prasada (2016): The effect of economic insecurity on mental health: Recent evidence from Australian panel data. In: Social science \& medicine (1982) 151, S. 250-258.

Rosenberg, Morris/Pearlin, Leonard I. (1978): Social Class and Self-Esteem Among Children and Adults. In: American Journal of Sociology 84, 1, S. 53-77.

Ross, Catherine E./Mirowsky, John (2006): Sex differences in the effect of education on depression: resource multiplication or resource substitution? In: Social science \& medicine (1982) 63, 5, S. 1400-1413.

Ross, Catherine E./Wu, Chia-Ling (1996): Education, Age, and the Cumulative Advantage in Health. In: Journal of Health and Social Behavior 37, 1, S. 104. 
Rözer, Jesper/Kraaykamp, Gerbert/Huijts, Tim (2016): National income inequality and self-rated health. The differing impact of individual social trust across 89 countries. In: European Societies 18, 3, S. 245-263.

Rözer, Jesper Jelle/Volker, Beate (2016): Does income inequality have lasting effects on health and trust? In: Social science \& medicine (1982) 149, S. 37-45.

Sapolsky, Robert M. (2004): Social Status and Health in Humans and Other Animals. In: Annual Review of Anthropology 33, 1, S. 393-418.

Sapolsky, Robert M. (2005): The Influence of Social Hierarchy on Primate Health. In: Science 308, 5722, S. 648-652.

Sareen, Jitender/Afifi, Tracie O./McMillan, Katherine A./Asmundson, Gordon J. G. (2011): Relationship between household income and mental disorders: findings from a population-based longitudinal study. In: Archives of general psychiatry 68, 4, S. 419-427.

Saß, Anke-Christine/Wurm, Susanne/Ziese, Thomas (2009): 2.1 Somatische und psychische Gesundheit. In: Robert Koch-Institut (Hrsg.): Gesundheitsberichterstattung des Bundes. Gesundheit und Krankheit im Alter. Berlin, S. 31-61.

Sass, Victoria/Kravitz-Wirtz, Nicole/Karceski, Steven M./Hajat, Anjum/Crowder, Kyle/Takeuchi, David (2017): The effects of air pollution on individual psychological distress. In: Health \& place 48, S. 72-79.

Schaan, Barbara (2013a): Social Determinants of Depression in Later Life. Doctoral dissertation: Universität Mannheim.

Schaan, Barbara (2013b): Widowhood and depression among older Europeans--the role of gender, caregiving, marital quality, and regional context. In: The journals of gerontology. Series B, Psychological sciences and social sciences 68, 3, S. 431442.

Schaan, Barbara (2014): The interaction of family background and personal education on depressive symptoms in later life. In: Social science \& medicine (1982) 102, S. 94-102.

Schaffer, Mark (2012): Probit better than LPM? http://www.mostlyharmlesseconometrics.com/2012/07/probit-better-than-lpm/ [Zugriff: 20.07.2018].

Scher, Christine D./Ingram, Rick E./Segal, Zindel V. (2005): Cognitive reactivity and vulnerability: empirical evaluation of construct activation and cognitive diatheses in unipolar depression. In: Clinical psychology review 25, 4, S. 487-510.

Scherr, Sebastian (2016): Depression - Medien - Suizid. Wiesbaden: Springer Fachmedien Wiesbaden.

Schindler, S./Schmidt, L./Stroske, M./Storch, M./Anwander, A./Trampel, R./Strauß, M./Hegerl, U./Geyer, S./Schönknecht, P. (2018): Hypothalamus enlargement in mood disorders. In: Acta psychiatrica Scandinavica.

Schneider, Brit S./Schneider, Udo (2009a): Determinants and consequences of health behaviour: New evidence from German micro data.

Schneider, Sven/Mohnen, Sigrid (2016): Der Einfluss der Wohnumgebung auf die Gesundheit - eine medizinsoziologische Betrachtung. In: Stauder, J./Rapp, I./Eckhard, J. (Hrsg.): Soziale Bedingungen privater Lebensführung. Wiesbaden: Springer Fachmedien Wiesbaden, S. 305-324. 
Schneider, Udo/Schneider, Brit S. (2009b): Willing to be healthy? On the health effects of smoking, drinking and an unbalanced diet. A multivariate probit approach. Diskussionspapier 01-09. Bayreuth.

Schnittker, Jason/McLeod, Jane D. (2005): The Social Psychology of Health Disparities. In: Annual Review of Sociology 31, 1, S. 75-103.

Schräpler, Jörg-Peter (2016): Respondent Behavior in Panel Studies. In: Sociological Methods \& Research 33, 1, S. 118-156.

Schubert, Christian (1998): Psychoneuroimmunologische Forschung im Kontext biochemischer Erkenntnisfortschritte und ihre paradigmatischen Grenzen. In: Zeitschrift für Psychosomatische Medizin und Psychoanalyse 44, 1, S. 1-20.

Schulz, Florian/Blossfeld, Hans-Peter (2006): Wie verändert sich die häusliche arbeitsteilung im eheverlauf? In: KZfSS Kölner Zeitschrift für Soziologie und Sozialpsychologie 58, 1, S. 23-49.

Schunck, Reinhard/Sauer, Carsten/Valet, Peter (2015): Unfair Pay and Health. The Effects of Perceived Injustice of Earnings on Physical Health. In: European Sociological Review 31, 6, S. 655-666.

Schütz, Johanna (2019): Marital Biography and Health in Old Age: Insights from European Survey Data. Heidelberg University Library. Heidelberg.

Schwandt, Hannes (2014): Wealth Shocks and Health Outcomes: Evidence fromStock Market Fluctuations. CEP Discussion Paper.

Seitz, Dallas/Purandare, Nitin/Conn, David (2010): Prevalence of psychiatric disorders among older adults in long-term care homes: a systematic review. In: International psychogeriatrics 22,7, S. 1025-1039.

Semyonov, Moshe/Lewin-Epstein, Noah/Maskileyson, Dina (2013): Where wealth matters more for health: the wealth-health gradient in 16 countries. In: Social science \& medicine (1982) 81, S. 10-17.

Sen, Amartya (1993): Capability and Well-Being. In: Nussbaum, M./Sen, A. (Hrsg.): The Quality of Life. Oxford University Press.

Sen, Amartya Kumar (1992): Inequality reexamined. New York: Russel Sage [u.a.].

Sexton, Claire E./Mackay, Clare E./Ebmeier, Klaus P. (2013): A systematic review and meta-analysis of magnetic resonance imaging studies in late-life depression. In: The American journal of geriatric psychiatry official journal of the American Association for Geriatric Psychiatry 21, 2, S. 184-195.

Shim, Joyce/Lee, RaeHyuck/Kim, Jaeseung (2016): Hard Times and Harder Minds. Material Hardship and Marital Well-Being Among Low-Income Families in South Korea. In: Journal of Family Issues 38, 18, S. 2545-2566.

Siddiqi, Arjumand/Jones, Marcella K./Erwin, Paul Campbell (2015): Does higher income inequality adversely influence infant mortality rates? Reconciling descriptive patterns and recent research findings. In: Social science \& medicine (1982) 131, S. 82-88.

Siegrist, Johannes (1995): Emotions and health in occupational life. New scientific findings and policy implications. In: Patient Education and Counseling 25, 3, S. 227-236.

Siegrist, Johannes (1996): Adverse health effects of high-effort/low-reward conditions. In: Journal of Occupational Health Psychology 1, 1, S. $27-41$. 
Siegrist, Johannes (2005): Medizinische Soziologie. 6., neu bearb. und erw. Aufl. München, Jena: Elsevier, Urban und Fischer.

Siegrist, Johannes/Peter, Richard (1996): Threat to occupational status control and cardiovascular risk. In: Israel journal of medical sciences 32, 3-4, S. 179-184.

Simonson, Julia/Gordo, Laura Romeu/Titova, Nadiya (2011): Changing employment patterns of women in Germany. How do baby boomers differ from older cohorts? A comparison using sequence analysis. In: Advances in Life Course Research 16, 2, S. 65-82.

Singh, Ankur/Harford, Jane/Antunes, José Leopoldo Ferreira/Peres, Marco A. (2018): Area-level income inequality and oral health among Australian adults-A population-based multilevel study. In: PloS one 13, 1, S. e0191438.

Skopek, Nora/Buchholz, Sandra/Blossfeld, Hans-Peter (2011): Wealth inequality in Europe and the delusive egalitarianism of Scandinavian countries. MPRA Paper.

Skopek, Nora/Buchholz, Sandra/Blossfeld, Hans-Peter (2014): National patterns of income and wealth inequality. In: International Journal of Comparative Sociology 55,6 , S. 463-488.

Skopek, Nora/Kolb, Kathrin/Buchholz, Sandra/Blossfeld, Hans-Peter (2012): Einkommensreich - vermögensarm? Die Zusammensetzung von Vermögen und die Bedeutung einzelner Vermögenskomponenten im europäischen Vergleich. In: Berliner Journal für Soziologie 22, 2, S. 163-187.

Slaets, Joris P. J. (2006): Vulnerability in the elderly: frailty. In: The Medical clinics of North America 90, 4, S. 593-601.

Slavich, George M./Irwin, Michael R. (2014): From stress to inflammation and major depressive disorder: a social signal transduction theory of depression. In: Psychological bulletin 140, 3, S. 774-815.

Smith, George Davey (1996): Income inequality and mortality: why are they related? In: BMJ: British Medical Journal 312, 20, S. 987-988.

Snelgrove, John W./Pikhart, Hynek/Stafford, Mai (2009): A multilevel analysis of social capital and self-rated health: evidence from the British Household Panel Survey. In: Social science \& medicine (1982) 68, 11, S. 1993-2001.

Sørensen, Kristine/Pelikan, Jürgen M./Röthlin, Florian/Ganahl, Kristin/Slonska, Zofia/Doyle, Gerardine/Fullam, James/Kondilis, Barbara/Agrafiotis, Demosthenes/Uiters, Ellen/Falcon, Maria/Mensing, Monika/Tchamov, Kancho/van den Broucke, Stephan/Brand, Helmut (2015): Health literacy in Europe: comparative results of the European health literacy survey (HLS-EU). In: European journal of public health 25, 6, S. 1053-1058.

Spannagel, Dorothee (2015): Das bedingungslose Grundeinkommen: Chancen und Risiken einer Entkoppelung von Einkommen und Arbeit. Hans-Böckler-Stiftung.

Spohn, Wolfgang (2012): The laws of belief: Ranking theory and its philosophical applications. Oxford University Press.

Statistisches Bundesamt (2018): Armutsgefährdungsquote in Deutschland nach Alter im Jahr 2016. https://de.statista.com/statistik/daten/studie/419433/umfrage/armutsgefaehrdungsquote-in-deutschland-nach-alter/ [Zugriff: 15.06.2018]. 
Stein, Petra/Bekalarczyk, Dawid (2017): Modelle mit variablen Regressionskonstanten. Random- und Fixed-Effects-Modelle. https://www.uni-due.de/imperia/md/content/soziologie/modelle_mit_variablen_regressionskonstanten.pdf [Zugriff: 18.07.2018].

Steptoe, Andrew/Kivimäki, Mika (2013): Stress and cardiovascular disease: an update on current knowledge. In: Annual review of public health 34, S. 337-354.

Stoppe, Gabriela (2008): Depressionen im Alter. In: Bundesgesundheitsblatt, Gesundheitsforschung, Gesundheitsschutz 51, 4, S. 406-410.

Strengmann-Kuhn, Wolfgang/Hauser, Richard (2012): International vergleichende Armutsforschung. In: Huster, E.-U./Boeckh, J./Mogge-Grotjahn, H. (Hrsg.): Handbuch Armut und Soziale Ausgrenzung. Wiesbaden: VS Verlag für Sozialwissenschaften, S. 164-183.

Stuck, Andreas E./Walthert, Jutta M./Nikolaus, Thorsten/Büla, Christophe J./Hohmann, Christoph/Beck, John C. (1999): Risk factors for functional status decline in community-living elderly people. A systematic literature review. In: Social science \& medicine 48, 4, S. 445-469.

Stuck, Stephanie/Zuber, Sabrina/Kotte, Markus/Franzese, Fabio/Gruber, Stefan/Birkenbach, Tim (2018): SHARE Release Guide 6.1.1, http://www.shareproject.org/fileadmin/pdf_documentation/SHARE_release_guide_6-1-1.pdf [Zugriff: 06.08.2018].

Sturm, R./Gresenz, C. R. (2002): Relations of income inequality and family income to chronic medical conditions and mental health disorders. National survey. In: BMJ 324, 7328, S. 20.

Subramanian, S. V./Kawachi, Ichiro (2004): Income inequality and health: what have we learned so far? In: Epidemiologic reviews 26, S. 78-91.

Subramanian, S. V./Kawachi, Ichiro (2006): Whose health is affected by income inequality? A multilevel interaction analysis of contemporaneous and lagged effects of state income inequality on individual self-rated health in the United States. In: Health \& place 12, 2, S. 141-156.

Subramanian, S.V/Kawachi, Ichiro/Kennedy, Bruce P. (2001): Does the state you live in make a difference? Multilevel analysis of self-rated health in the US. In: Social science \& medicine 53, 1, S. 9-19.

Sun, Xiangyu/Bernabé, Eduardo/Liu, Xuenan/Gallagher, Jennifer E./Zheng, Shuguo (2017): Do contextual factors have a role in periodontal disease? In: Journal of clinical periodontology 44, 1, S. 13-21.

Szanton, S. L./Allen, J. K./Thorpe, R. J./Seeman, T./Bandeen-Roche, K./Fried, L. P. (2008): Effect of Financial Strain on Mortality in Community-Dwelling Older Women. In: The Journals of Gerontology Series B: Psychological Sciences and Social Sciences 63, 6, S. S369-S374.

Szanton, Sarah L./Thorpe, Roland J./Whitfield, Keith (2010): Life-course financial strain and health in African-Americans. In: Social science \& medicine (1982) 71, 2, S. 259-265.

Tabassum, Faiza/Kumari, Meena/Rumley, Ann/Lowe, Gordon/Power, Chris/Strachan, David P. (2008): Effects of socioeconomic position on inflammatory and hemostatic markers: a life-course analysis in the 1958 British birth cohort. In: American journal of epidemiology 167, 11, S. 1332-1341. 
Tak, Erwin/Kuiper, Rebecca/Chorus, Astrid/Hopman-Rock, Marijke (2013): Prevention of onset and progression of basic ADL disability by physical activity in community dwelling older adults: a meta-analysis. In: Ageing research reviews 12, 1 , S. 329-338.

Taylor, Mark P./Pevalin, David J./Todd, Jennifer (2007): The psychological costs of unsustainable housing commitments. In: Psychological medicine 37, 7, S. 10271036.

Taylor, Peter James/Gooding, Patricia/Wood, Alex M./Tarrier, Nicholas (2011): The role of defeat and entrapment in depression, anxiety, and suicide. In: Psychological bulletin 137, 3, S. 391-420.

Taylor, W. D./Aizenstein, H. J./Alexopoulos, G. S. (2013): The vascular depression hypothesis: mechanisms linking vascular disease with depression. In: Molecular psychiatry 18, 9, S. 963-974.

Terraneo, Marco (2017): Material Deprivation, Health and Well-Being in Older Age: A Comparative Analysis of 14 European Countries. In: White, R. (Hrsg.): Measuring multidimensional poverty and deprivation. Incidence and determinants in developed countries. Global perspectives on wealth and distribution. Cham: Palgrave Macmillan, S. 113-137.

Thaler, Richard (1980): Toward a positive theory of consumer choice. In: Journal of Economic Behavior \& Organization 1, 1, S. 39-60.

Theorell, Töres/Karasek, Robert A. (1996): Current issues relating to psychosocial job strain and cardiovascular disease research. In: Journal of Occupational Health Psychology 1, 1, S. 9-26.

Thomas, Tobias/Diermeier, Matthias/Goecke, Henry/Niehues, Judith (2018): Einkommensungleichheit, wahrgenommene Ungleichheit und der Einfluss der Medien. In: Wirtschaftspolitische Blätter, 1, S. 39-64.

Thomson, Cynthia A./Giuliano, Anna/Rock, Cheryl L./Ritenbaugh, Cheryl K./Flatt, Shirley W./Faerber, Susan/Newman, Vicky/Caan, Bette/Graver, Ellen/Hartz, Vern/Whitacre, Robin/Parker, Felicia/Pierce, John P./Marshall, James R. (2003): Measuring Dietary Change in a Diet Intervention Trial. Comparing Food Frequency Questionnaire and Dietary Recalls. In: American journal of epidemiology 157, 8, S. 754-762.

Tjepkema, Michael/Wilkins, Russell/Long, Andrea (2013): Cause-specific mortality by income adequacy in Canada: A 16-year follow-up study. In: Health Reports 24, 7, S. 14-22.

Topinková, Eva (2008): Aging, disability and frailty. In: Annals of nutrition \& metabolism 52 Suppl 1, S. 6-11.

Torre, Roberta/Myrskylä, Mikko (2014): Income inequality and population health: an analysis of panel data for 21 developed countries, 1975-2006. In: Population studies 68,1, S. $1-13$.

Townsend, Peter (1979): Poverty in the United Kingdom. A survey of household resources and standards of living. London: Allen Lane and Penguin Books.

Tsimbos, Cleon/Verropoulou, Georgia (2008): A multivariate analysis of factors associated with physical functioning limitations in the Greek elderly population. In: Journal of Statistics and Management Systems 11, 6, S. 1161-1180. 
Tsutsumi, Akizumi/Kawakami, Norito (2004): A review of empirical studies on the model of effort-reward imbalance at work: reducing occupational stress by implementing a new theory. In: Social science \& medicine (1982) 59, 11, S. 2335-2359.

Tsutsumi, Akizumi/Kayaba, Kazunori/Theorell, Töres/Siegrist, Johannes (2001): Association between job stress and depression among Japanese employees threatened by job loss in a comparison between two complementary job-stress models. In: Scandinavian journal of work, environment \& health 27, 2, S. 146-153.

Tucker-Seeley, Reginald D./Harley, Amy E./Stoddard, Anne M./Sorensen, Glorian G. (2013): Financial hardship and self-rated health among low-income housing residents. In: Health education \& behavior the official publication of the Society for Public Health Education 40, 4, S. 442-448.

Tur-Sinai, Aviad/Litwin, Howard (2015): Forgone visits to the doctor due to cost or lengthy waiting time among older adults in Europe. In: Börsch-Supan, A./Kneip, T./Litwin, H./Myck, M./Weber, G. (Hrsg.): Ageing in Europe - Supporting Policies for an Inclusive Society. Berlin, München, Boston: DE GRUYTER, S. 291300.

Turvey, Carolyn L./Conwell, Yeates/Jones, Michael P./Phillips, Caroline/Simonsick, Eleanor/Pearson, Jane L./Wallace, Robert (2002): Risk Factors for Late-Life Suicide. A Prospective, Community-Based Study. In: The American Journal of Geriatric Psychiatry 10, 4, S. 398-406.

Tversky, A./Kahneman, D. (1991): Loss Aversion in Riskless Choice. A ReferenceDependent Model. In: The Quarterly Journal of Economics 106, 4, S. 1039-1061.

Twenge, Jean M./Campbell, W. Keith (2016): Self-Esteem and Socioeconomic Status. A Meta-Analytic Review. In: Personality and Social Psychology Review 6, 1, S. 59-71.

Uliaszek, Amanda A./Zinbarg, Richard E./Mineka, Susan/Craske, Michelle G./Sutton, Jonathan M./Griffith, James W./Rose, Raphael/Waters, Allison/Hammen, Constance (2010): The role of neuroticism and extraversion in the stress-anxiety and stress-depression relationships. In: Anxiety, stress, and coping 23, 4, S. 363-381.

United Nations (2017): Sustainable Development Goals Report 2017. New York: United Nations.

Unützer, Jürgen/Katon, Wayne/Callahan, Christopher M./Williams, John W./Hunkeler, Enid/Harpole, Linda/Hoffing, Marc/Della Penna, Richard D./Noel, Polly Hitchcock/Lin, Elizabeth H. B./Tang, Lingqi/Oishi, Sabine (2003): Depression Treatment in a Sample of 1,801 Depressed Older Adults in Primary Care. In: Journal of the American Geriatrics Society 51, 4, S. 505-514.

Uslaner, Eric M./Brown, Mitchell (2005): Inequality, Trust, and Civic Engagement. In: American Politics Research 33, 6, S. 868-894.

van der Heide, Iris/Rademakers, Jany/Schipper, Maarten/Droomers, Mariël/Sørensen, Kristine/Uiters, Ellen (2013): Health literacy of Dutch adults: a cross sectional survey. In: BMC public health 13, S. 179.

van Deurzen, Ioana/van Ingen, Erik/van Oorschot, Wim J. H. (2015): Income Inequality and Depression. The Role of Social Comparisons and Coping Resources. In: European Sociological Review 31, 4, S. 477-489. 
van Deurzen, Ioana/van Oorschot, Wim/van Ingen, Erik (2014): The link between inequality and population health in low and middle income countries: policy myth or social reality? In: PloS one 9, 12.

Venn, Danielle/Strazdins, Lyndall (2017): Your money or your time? How both types of scarcity matter to physical activity and healthy eating. In: Social science \& medicine (1982) 172, S. 98-106.

Verbrugge, Lois M./Jette, Alan M. (1994): The disablement process. In: Social science \& medicine 38,1, S. 1-14.

Videbech, Poul/Ravnkilde, Barbara (2004): Hippocampal volume and depression: A metaanalysis of MRI studies. In: American Journal of Psychiatry 161, 11, S. 19571966.

Vigelsø, Andreas/Gram, Martin/Wiuff, Caroline/Andersen, Jesper L./Helge, Jørn W./Dela, Flemming (2015): Six weeks' aerobic retraining after two weeks' immobilization restores leg lean mass and aerobic capacity but does not fully rehabilitate leg strength in young and older men. In: Journal of rehabilitation medicine 47, 6, S. 552-560.

Vink, Dagmar/Aartsen, Marja J./Schoevers, Robert A. (2008): Risk factors for anxiety and depression in the elderly: a review. In: Journal of Affective Disorders 106, 12, S. 29-44.

Visser, M./Goodpaster, B. H./Kritchevsky, S. B./Newman, A. B./Nevitt, M./Rubin, S. M./Simonsick, E. M./Harris, T. B. (2005): Muscle Mass, Muscle Strength, and Muscle Fat Infiltration as Predictors of Incident Mobility Limitations in WellFunctioning Older Persons. In: The Journals of Gerontology Series A: Biological Sciences and Medical Sciences 60, 3, S. 324-333.

Vries, Robert de/Blane, David/Netuveli, Gopalakrishnan (2014): Long-term exposure to income inequality: implications for physical functioning at older ages. In: European journal of ageing 11, 1, S. 19-29.

Waern, Margda/Rubenowitz, Eva/Wilhelmson, Katarina (2003): Predictors of suicide in the old elderly. In: Gerontology 49, 5, S. 328-334.

Wagstaff, A./van Doorslaer, E. (2000): Income inequality and health: what does the literature tell us? In: Annual review of public health 21, S. 543-567.

Wahrendorf, Morten/Reinhardt, Jan D./Siegrist, Johannes (2013): Relationships of disability with age among adults aged 50 to 85: evidence from the United States, England and continental europe. In: PloS one 8, 8, S. e71893.

Waldron, Hilary (2013): Mortality Differentials by Lifetime Earnings Decile: Implications for Evaluations of Proposed Social Security Law Changes. In: Social Security Bulletin 73, 1, S. 1-37.

Walker, Robert/Kyomuhendo, Grace Bantebya/Chase, Elaine/Choudhry, Sohail/Gubrium, Erika K./Nicola, J. Yongmieo/Lødemel, Ivar/Mathew, Leemamol/MwiiineE, Amon/Pellissery, Sony/Ming, Y. A.N. (2013): Poverty in Global Perspective. Is Shame a Common Denominator? In: Journal of Social Policy 42, 02, S. 215-233.

Warren, Tracey (2010): Penalties of Part-time Work Across Europe. In: Scott, J./Crompton, R./Lyonette, C. (Hrsg.): Gender Inequalities in the 21 st Century. Edward Elgar Publishing, S. 109-125. 
Waters, Lea E./Moore, Kathleen A. (2002): Predicting self-esteem during unemployment. The effect of gender, financial deprivation, alternate roles, and social support. In: Journal of Employment Counseling 39, 4, S. 171-189.

Wedegärtner, Felix/Arnhold-Kerri, Sonja/Sittaro, Nicola-Alexander/Lohse, Ralf/Dietrich, Detlef E./Bleich, Stefan/Geyer, Siegfried (2011): Invalidität und Tod bei Erwerbsfähigen mit Depressionen. In: Psychiatrische Praxis 38, 3, S. 135-141.

Weich, Scott/Lewis, Glyn/Jenkins, Stephen P. (2001): Income inequality and the prevalence of common mental disorders in Britain. In: British Journal of Psychiatry 178,03 , S. 222-227.

Weisser, Gerhard (1957): Einige Grundbegriffe der Sozialpolitiklehre. Archiv der sozialen Demokratie. Nachlass Gerhard Weisser. Akte 842. Bonn.

Wendt, Eva Diana (2010): Sozialer Abstieg und Konsum. Auswirkungen finanzieller Verknappung auf das Konsumverhalten. 1. Auflage. Gabler Research MarketingManagement. Wiesbaden: Gabler.

Wengler, Annelene/Trappe, Heike/Schmitt, Christian (2008): Partnerschaftliche Arbeitsteilung und Elternschaft. Analysen zur Aufteilung von Hausarbeit und Elternaufgaben auf Basis des Generations and Gender Survey. Materialien zur Bevölkerungswissenschaft. Wiesbaden.

Westendorp, Rudi G. J./Kirkwood, Thomas B. L. (2008): The biology of ageing. In: Bond, J./Peace, S./Dittmann-Kohli, F./Westerhof, G. J. (Hrsg.): Ageing in society. European perspectives on gerontology. 3rd ed., reprinted. London, Los Angeles: Sage Publications, S. 15-37.

Westerhof, Gerben J./Tulle/Emmanuelle (2008): Meanings of ageing and old age: discursive contexts, social attitudes and personal identities. In: Bond, J./Peace, S./Dittmann-Kohli, F./Westerhof, G. J. (Hrsg.): Ageing in society. European perspectives on gerontology. 3rd ed., reprinted. London, Los Angeles: Sage Publications, S. 235-254.

Whitbeck, Les B./Simons, Ronald L./Conger, Rand D./Lorenz, Frederick O./Huck, Shirley/Elder, Glenn H. (1991): Family Economic Hardship, Parental Support, and Adolescent Self-Esteem. In: Social Psychology Quarterly 54, 4, S. 353.

Wilcox, Sara/Evenson, Kelly R./Aragaki, Aaron/Wassertheil-Smoller, Sylvia/Mouton, Charles P./Loevinger, Barbara Lee (2003): The effects of widowhood on physical and mental health, health behaviors, and health outcomes: The Women's Health Initiative. In: Health psychology official journal of the Division of Health Psychology, American Psychological Association 22, 5, S. 513-522.

Wilhelmsson, Mats (2000): The Impact of Traffic Noise on the Values of Single-family Houses. In: Journal of Environmental Planning and Management 43, 6, S. 799815.

Wilkinson, Richard/Pickett, Kate (2009a): The spirit level. Why more equal societies almost always do better. London: Allen Lane.

Wilkinson, Richard G. (1994): The Epidemiological Transition: From Material Scarcity to Social Disadvantage? In: Daedalus 123, 4, S. 61-77.

Wilkinson, Richard G. (1996): Unhealthy societies: the afflictions of inequality. London: Routledge. 
Wilkinson, Richard G./Pickett, Kate E. (2006): Income inequality and population health: a review and explanation of the evidence. In: Social science \& medicine (1982) 62, 7, S. 1768-1784.

Wilkinson, Richard G./Pickett, Kate E. (2008): Income inequality and socioeconomic gradients in mortality. In: American journal of public health 98, 4, S. 699-704.

Wilkinson, Richard G./Pickett, Kate E. (2009b): Income Inequality and Social Dysfunction. In: Annual Review of Sociology 35, 1, S. 493-511.

Wilkinson, Richard G./Pickett, Kate E. (2017): The enemy between us. The psychological and social costs of inequality. In: European Journal of Social Psychology 47, 1, S. 11-24.

Williams, Kristi/Frech, Adrianne/Carlson, Daniel L. (2010): Marital Status and Mental Health. In: Scheid, T. L./Brown, T. N. (Hrsg.): A handbook for the study of mental health. Social contexts, theories, and systems. 2nd ed. Cambridge, New York: Cambridge University Press, S. 306-320.

Wittchen, Hans-Ulrich/Jacobi, Frank/Klose, Michael/Ryl, Livia (2010): Depressive Erkrankungen. Gesundheitsberichterstattung des Bundes. Berlin.

Wolf, Elke (2002): Lower wage rates for fewer hours? A simultaneous wage-hours model for Germany. In: Labour Economics 9, 5, S. 643-663.

Wolff, Julia K./Tesch-Römer, Clemens (2017): Glücklich bis ins hohe Alter? Lebenszufriedenheit und depressive Symptome in der zweiten Lebenshälfte. In: Mahne, K./Wolff, J. K./Simonson, J./Tesch-Römer, C. (Hrsg.): Altern im Wandel. Wiesbaden: Springer Fachmedien Wiesbaden, S. 171-183.

Wood, Alex M./Boyce, Christopher J./Moore, Simon C./Brown, Gordon D. A. (2012): An evolutionary based social rank explanation of why low income predicts mental distress: a 17 year cohort study of 30,000 people. In: Journal of Affective Disorders 136,3 , S. $882-888$.

Wooldridge, Jeffrey M. (2002): Econometric analysis of cross section and panel data. Cambridge: MIT Press.

World Bank (2017): Monitoring global poverty: Report of the commission on global poverty. Washington: World Bank.

World Health Organization (2000): The implications for training of embracing. A life course approach to health.

World Health Organization (2007): Global Age-friendly Cities: A Guide. Genf.

World Health Organization (2012): Depression in Europe. http://www.euro.who.int/en/ health-topics/noncommunicable-diseases/pages/news/news/2012/10/depressionin-europe [Zugriff: 25.10.2018].

World Health Organization (2017a): Depression. http://www.who.int/topics/depression/en/ [Zugriff: 19.06.2017].

World Health Organization (2017b): Depression. Fact sheet. http://www.who.int/mediacentre/factsheets/fs369/en/ [Zugriff: 08.03.2017].

World Health Organization (2017c): Depression and Other Common Mental Disorders: Global Health Estimates.

World Health Organization (2017d): Water and sanitation. Data and statistics. http:// www.euro.who.int/en/health-topics/environment-and-health/water-and-sanitation/data-and-statistics [Zugriff: 13.03.2017]. 
Wright, Matthew R./Brown, Susan L. (2017): Psychological Well-Being among Older Adults: The Role of Partnership Status. In: Journal of marriage and the family 79, 3, S. 833-849.

Yang, Yang (2006): How does functional disability affect depressive symptoms in late life? The role of perceived social support and psychological resources. In: Journal of Health and Social Behavior 47, 4, S. 355-372.

Yeh, S.-R./Fricke, R. A./Edwards, D.H. (1996): The Effect of Social Experience on Serotonergic Modulation of the Escape Circuit of Crayfish. In: Science 271, 5247, S. 366-369.

Zak, Paul J./Knack, Stephen (2001): Trust and Growth. In: The Economic Journal 111, 470, S. 295-321.

Zheng, Hui (2012): Do people die from income inequality of a decade ago? In: Social science \& medicine (1982) 75, 1, S. 36-45.

Zimmerman, Frederick J./Katon, Wayne (2005): Socioeconomic status, depression disparities, and financial strain: what lies behind the income-depression relationship? In: Health economics 14, 12, S. 1197-1215. 


\section{utb.}

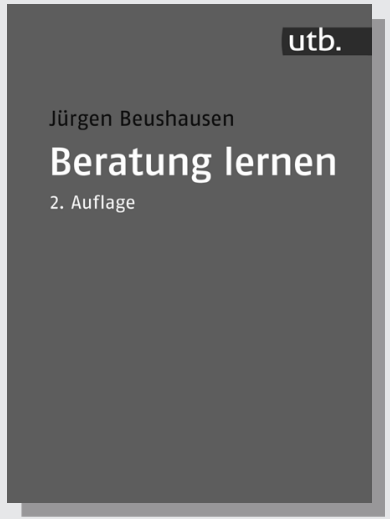

Jürgen Beushausen

Beratung lernen

Grundlagen Psychosozialer Beratung und Sozialtherapie für Studium und Praxis

utb $M$

2., überarbeitete Aufl. 2020 • 396 Seiten • Kart. • 25,00€ (D) • 25,80€ (A) ISBN 978-3-8252-5323-3 • eISBN 978-3-8385-5323-8

Das Lehrbuch bietet eine umfassende Einführung in integrative und systemische Beratungskonzepte, um praxisnah die eigenen Beratungskompetenzen zu entwickeln. Beratungskompetenz ist eine grundlegende Handlungsorientierung für alle psychosozialen Arbeitsfelder - das Buch liefert das Grundwissen sowie direkte Umsetzungsstrategien, um die nötigen Fähigkeiten zu entwickeln und diese angemessen und kompetent anzuwenden. Beigefügte Reflexionsbögen, Seminarfragen und Arbeitsblätter komplettieren das Handwerkszeug.

\section{www.utb-shop.de}


Armutsrisiko

alleinerziehend

Die Bedeutung von sozialer

Komposition und institutionellem

Kontext in Deutschland

\section{Armutsrisiko alleinerziehend}

Die Bedeutung von sozialer Komposition und institutionellem Kontext in Deutschland

2020. 309 Seiten $•$ Kart. $• 61,00 €(D) \cdot 62,80 €(A)$

ISBN 978-3-86388-818-3 • eISBN 978-3-86388-448-2

Der Titel Armutsrisiko alleinerziehend (DOI: 10.3224/86388818) ist kostenlos im Open Access (PDF) herunterladbar oder kostenpflichtig als Print-Ausgabe erhältlich.

Das Buch untersucht die Ursachen für das hohe Armutsrisiko alleinerziehender Mütter in Deutschland. Es werden sowohl die Familienund Erwerbsverläufe als auch der institutionelle Kontext betrachtet. In quantitativen Analysen werden der armutsverstärkende Effekt der sozialen Komposition und des Alleinerziehens identifiziert. Anhand eines Periodenvergleichs und eines Vergleichs mit UK wird der Einfluss des institutionellen Kontexts quantifiziert. 


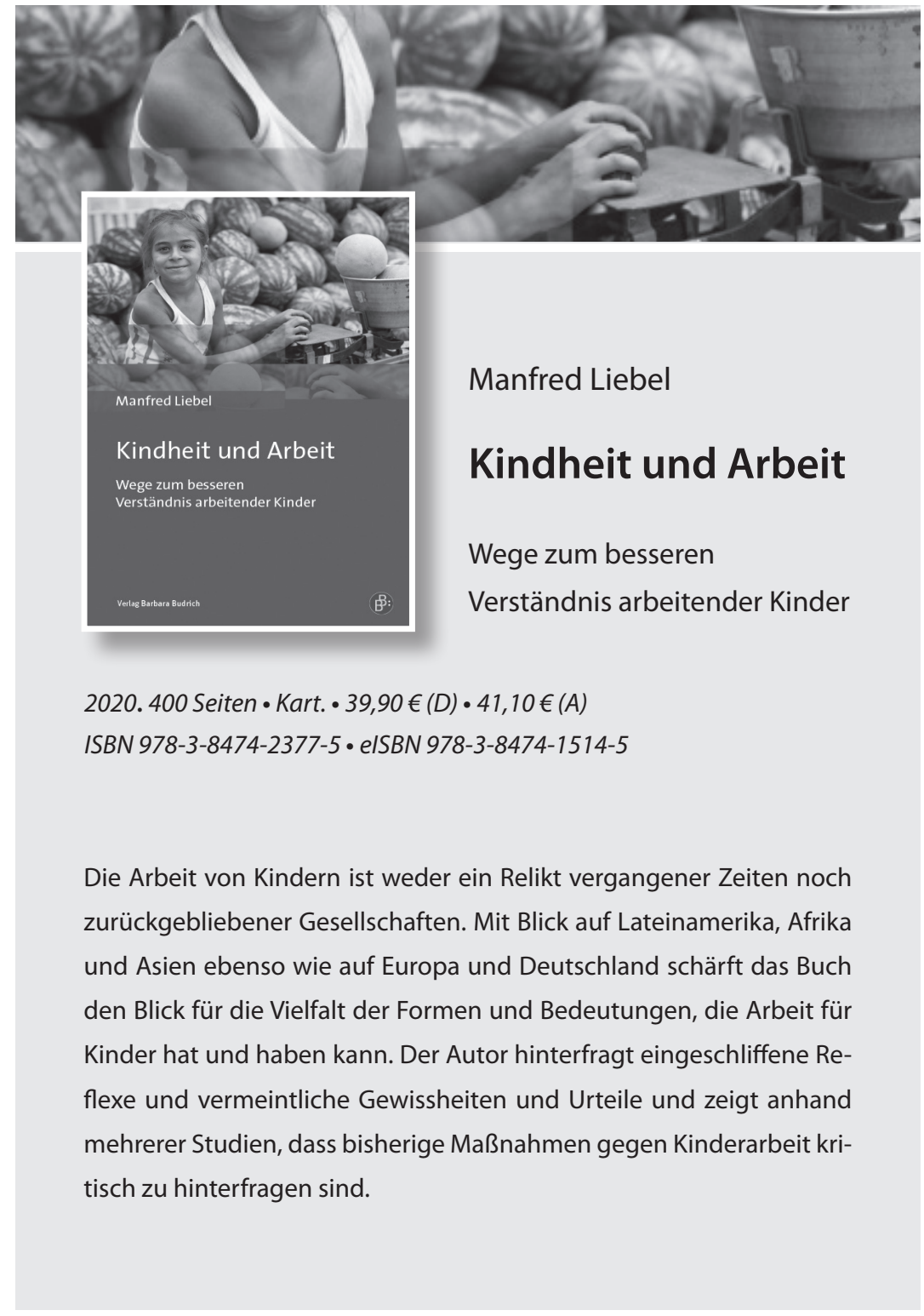

www.shop.budrich.de 


\section{Fabio Franzese Mentale und physische Gesundheit im Alter}

Der Einfluss von Armut, Einkommensungleichheit und Vermögensungleichheit

Wie wirken sich Veränderungen in der finanziellen Lage auf verschiedene Dimensionen der Gesundheit im Alter aus? In empirischen Analysen mit Längsschnittdaten des Survey of Health, Ageing and Retirement in Europe (SHARE) untersucht der Autor verschiedene Dimensionen der Gesundheit: Depression, subjektive Gesundheit und körperliche Einschränkungen. Zudem werden die Einflüsse von Einkommens- und Vermögensungleichheit in der Gesellschaft auf die individuelle Gesundheit erforscht.

Der Autor: Fabio Franzese, wissenschaftlicher Mitarbeiter, Max-Planck-Institut für Sozialrecht und Sozialpolitik, München

ISBN 978-3-96665-012-0

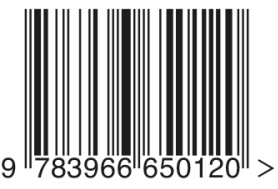

